
of plants to thrips feeding

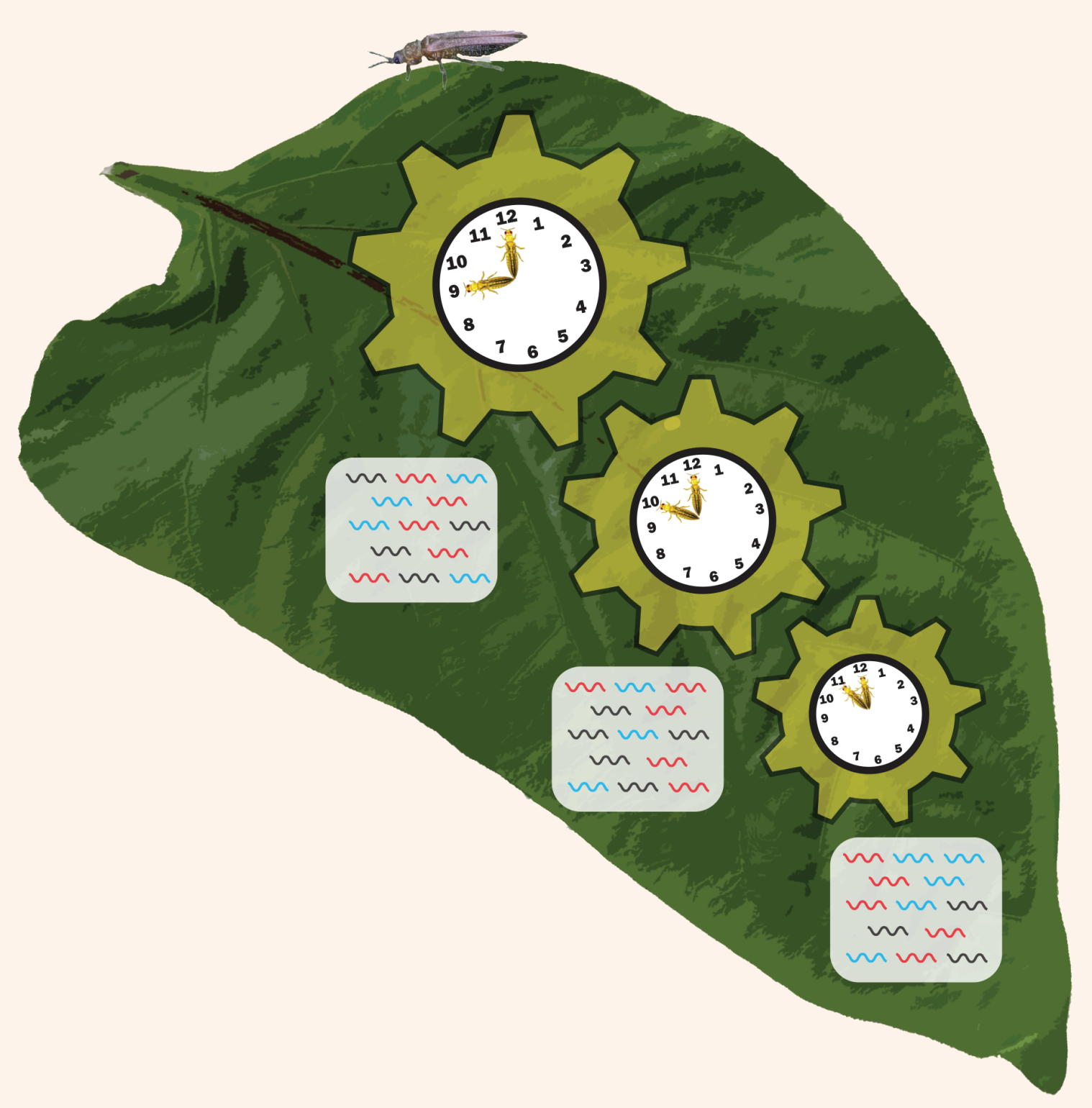




\section{Propositions}

1. Transcriptional responses of plants have more similarities in upregulated genes than in downregulated genes.

(this thesis)

2. Translation of research from model to non-model plants is a myth. (this thesis)

3. In science, analytical advances are more needed than technological advances.

4. We are sinking in data and starving for knowledge.

5. Nature has its own programming script.

6. To live in dignified poverty is better than to live in undignified wealth.

7. Accomplishment is in pursuing excellence and not success.

Propositions belonging to the thesis entitled,

"Dynamics of transcriptional responses of plants to thrips feeding"

Sandeep J. Sarde

Wageningen, 20th June 2019 


\title{
Dynamics of transcriptional responses of plants to thrips feeding
}

\author{
Sandeep J. Sarde
}




\section{Thesis committee}

\section{Promotor}

Prof. Dr Marcel Dicke

Professor of Entomology

Wageningen University \& Research

\section{Other members}

Prof. Dr Dick de Ridder, Wageningen University \& Research

Prof. Dr Ton Bisseling, Wageningen University \& Research

Dr Geert Smant, Wageningen University \& Research

Dr Colette Broekgaarden, Keygene, Wageningen

This research was conducted under the auspices of the Graduate School of Experimental Plant Sciences 


\title{
Dynamics of transcriptional responses of plants to thrips feeding
}

\author{
Sandeep J. Sarde
}

\section{Thesis}

submitted in fulfilment of the requirements for the degree of doctor at Wageningen University by the authority of the Rector Magnificus,

Prof. Dr A.P.J. Mol, in the presence of the

Thesis Committee appointed by the Academic Board to be defended in public on Thursday 20 June 2019 at 11 a.m. in the Aula. 


\section{Sandeep J. Sarde}

Dynamics of transcriptional responses of plants to thrips feeding, 180 pages.

PhD thesis, Wageningen University, Wageningen, the Netherlands (2019) With references, with a summary in English

DOI: $10.18174 / 476979$

ISBN: 978-94-6343-973-2 
To my beloved family, 



\section{Table of contents}

Chapter 1

General introduction

\section{Chapter 2}

Genome-wide identification, classification and expression of lipoxygenase gene family in pepper

SJ. Sarde, A. Kumar, RN. Remme and M. Dicke

Plant Molecular Biology 2018

Chapter 3

Involvement of sweet pepper CaLOX2 in jasmonate-dependent induced defence against Western flower thrips

SJ Sarde, K. Bouwmeester, J. Venegas-Molina, A. David, W. Boland, M. Dicke Journal of Integrative Plant Biology 2018

\section{Chapter 4}

Whole-genome transcriptional reprogramming of sweet pepper in response to Western flower thrips feeding

SJ. Sarde, R. Hickman, M. Steenbergen, G. Wiegers, CMJ. Pieterse, SCM. van Wees and M. Dicke

\section{Chapter 5}

Comparative high-density transcriptomics reveals rapid and complex rearrangement of white cabbage transcriptome in response to thrips feeding SJ. Sarde, R. Hickman, M. Steenbergen, G. Wiegers, CMJ. Pieterse,

SCM. van Wees and M. Dicke

Chapter 6

General discussion

Summary

Acknowledgements

Curriculum vitae

List of publications

Education Statement 



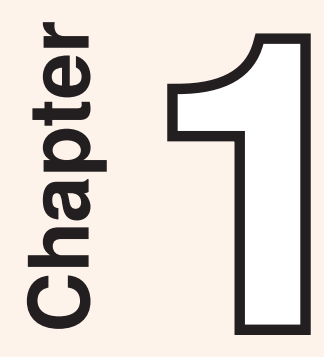

General introduction 



\section{Plants and herbivorous insects}

Interactions between plants and herbivorous insects have existed for more than 400 million years (Labandeira, 2007). To combat herbivorous insects, plants have evolved two layers of defences, constitutive and induced defences. Constitutive defences are always present in the plants, whereas induced defences are elicited in response to external stimuli such as chemical, physical or (a)biotic stresses. Induced defences can be further categorized into direct and indirect defences. Direct defences are plant traits that influence the performance of herbivorous insects (Howe \& Jander, 2008; War et al., 2012). In contrast, indirect defences promote the enemies of herbivores, e.g. by the release of a cocktail of volatile compounds, termed volatile organic compounds (VOCs) or herbivore-induced plant volatiles (HIPVs), that attract natural enemies (predators and parasitoids) of the attacking herbivorous insects (Dicke, 2009; McCormick et al., 2012; Mithofer \& Boland, 2012; Dicke, 2015).

Furthermore, plants perceive herbivorous insects via damage-associated molecular patterns (DAMPs) and herbivore associated molecular patterns (HAMPs) (Mithofer \& Boland, 2008; Heidel-Fischer et al., 2014; Duran-Flores \& Heil, 2016; Hilker \& Fatouros, 2016). The perception of herbivores can depend on, 1) mode of insect feeding and 2) the specific effectors, derived from insect saliva or eggs (Heidel-Fischer et al., 2014). Effector proteins are produced by herbivorous insects in their saliva that can interfere with and regulate the induced plant defences to the benefit of the herbivores (Hogenhout \& Bos, 2011; Kant et al., 2015; Giron et al., 2016). Moreover, based on the mode of feeding, herbivorous insects can broadly be classified into three major groups, 1) chewers like caterpillars, 2) sap feeders like aphids and whiteflies and 3) cell-content feeders like spider-mites and thrips (Stam et al., 2014). Ample knowledge on different elements of induced plant defences such as HAMPs (Douglas, 2018), effector proteins and resistance genes (Hogenhout \& Bos, 2011; Reymond, 2013; Douglas, 2018) is available for leaf chewers and phloem feeders, but not for cell-content feeders.

\section{Induced plant defences against herbivores}

Early recognition of an attacking herbivore can be a key factor for a plant's success in defending itself. Induced plant defences against herbivores can be categorized into three main phases: recognition, signalling and response (Heidel-Fischer et al., 2014). At the plant-insect interface, plants recognize herbivorous insects through specific insect-inflicted damage or insect-associated specific chemical cues (Mithofer \& Boland, 2008; Heidel-Fischer et al., 2014; Duran-Flores \& Heil, 2016; Hilker \& Fatouros, 2016). Subsequent to herbivore recognition, a series of early and late (in timescale of seconds to days) events occurs in plants (Maffei et al., 2007). The earliest mechanism (timescale of seconds to minutes) is an alteration in plasma membrane potential 
$\left(\mathrm{V}_{\mathrm{m}}\right)$, followed by variation in cytosolic $\mathrm{Ca}^{2+}$ concentrations and the biosynthesis of $\mathrm{H}_{2} \mathrm{O}_{2}$ (Maffei et al., 2007). Consequently, at a timescale of minutes, kinases and phytohormones are induced, altering the expression of transcripts (timescale of minutes to hours) and the biosynthesis of metabolites (timescale of hours to days) (Maffei et al., 2007; Stam et al., 2014). The temporal rearrangements of the transcriptome form the basis of extensive changes occurring in plant phenotype through the activation and deactivation of various biological processes (Stam et al., 2014). This dynamic change in phenotype can influence the entire ecology of plants, throughout the season (Poelman et al., 2010) or over different seasons (Stam et al., 2018) and plant interactions with insects at different trophic levels (Stam et al., 2014).

Plant hormones, such as jasmonic acid (JA), ethylene (ET) and salicylic acid (SA), are major players in regulating defences in response to feeding by different insect herbivores (Pieterse et al., 2009; Verhage et al., 2010; Pieterse et al., 2012; Stam et al., 2014). For example, JA especially mediates responses induced by chewing insects like caterpillars (Reymond et al., 2004; De Vos et al., 2005) and cell-content feeding insects like thrips (Abe et al., 2008; Abe et al., 2009; Steenbergen et al., 2018), whereas SA mediates responses to phloem-feeding insects like aphids and whiteflies (Zhu-Salzman et al., 2004; Walling, 2008; Pieterse et al., 2012; Tzin et al., 2015; Broekgaarden et al., 2018). Ethylene (ET) often acts synergistically with $\mathrm{JA}$ to fine-tune induced plant defences against herbivorous insects (Pieterse et al., 2009; Pieterse et al., 2012; Stam et al., 2014). Besides, other plant hormones such as gibberllins, abscisic acid (ABA), cytokinins and auxins are known to play a role in responses to herbivory. The regulation of induced defences to herbivory by these plant hormones extensively shapes the plant phenotype.

\section{Transcriptional responses of plants to herbivore feeding}

Several studies have captured whole-genome transcriptional responses of Arabidopsis against different insect herbivores using microarray or RNA-Seq platforms. Whole-genome microarray studies have been performed in response to caterpillars (Reymond et al., 2004; De Vos et al., 2005; Ehlting et al., 2008; Bidart-Bouzat \& Kliebenstein, 2011; Zhang et al., 2013; Appel et al., 2014; Coolen et al., 2016; Davila Olivas et al., 2016; Kroes et al., 2017), whiteflies (Kempema et al., 2007; Zhang et al., 2013) and aphid infestation (De Vos et al., 2005; Bidart-Bouzat \& Kliebenstein, 2011; Kroes et al., 2017). Reymond et al. (2004) reported that $67-84 \%$ of the total transcriptional response of Arabidopsis to Pieris rapae feeding is regulated by JA. Similarly, microarray or RNA-Seq platforms were used to capture whole-transcriptome profiles of non-model plant species in response to feeding by different insect herbivores. For instance, wild cabbage response to caterpillars (Broekgaarden et al., 2011b) or whiteflies (Broekgaarden et al., 2018), maize response to aphids (Tzin et 
al., 2015) and tomato, maize, barley and grapevine to spider mites (Martel et al., 2015; Diaz-Riquelme et al., 2016; Bui et al., 2018). The limitation of these studies on different insect herbivores is that the plant transcriptional response assessment is based on one or two time points over a period of 24 hours or longer (De Vos et al., 2005; Ehlting et al., 2008; Bidart-Bouzat \& Kliebenstein, 2011; Zhang et al., 2013; Appel et al., 2014; Diaz-Riquelme et al., 2016; Kroes et al., 2017; Broekgaarden et al., 2018; Tu et al., 2018) with the exception of a study that comprised six time points for the response of wild tobacco plants to Manduca sexta oral secretion application, over a time span of 13 hours (Durrant et al., 2017).

Several recent studies have shown that a plant dynamically reconfigures its transcriptome with time (Breeze et al., 2011; Windram et al., 2012; Hickman et al., 2017). However, the transcriptional responses captured in response to different insect herbivores present low-resolution portraits of transcriptional responses, as the studies were performed with limited time points (one or two time points in a period of 24 hours or longer) (De Vos et al., 2005; Ehlting et al., 2008; Bidart-Bouzat \& Kliebenstein, 2011; Zhang et al., 2013; Appel et al., 2014; Diaz-Riquelme et al., 2016; Kroes et al., 2017; Broekgaarden et al., 2018; Tu et al., 2018). Therefore, generation of high-resolution time-series transcriptional data in response to insect herbivory will result in a more detailed understanding of the temporal transcriptional reprogramming of plants. Thorough quantification of transcripts over several time points can elucidate the chronology of biological pathways (phytohormones, secondary metabolites or developmental processes), involvement of major transcription factors (TFs) and building a platform to construct gene regulatory networks. In addition, performing comparative transcriptomics using the high-resolution transcriptional data of different plant species in response to insect herbivory can identify commonalities and specificities of induced responses on plant-species or plant-family level. Moreover, this comparison can also unravel other aspects, such as relative response time of different plant species, complexity of transcriptional response and order of changes in biological events.

JA has been identified as a central player in modulating defences against several arthropod herbivores (Pieterse et al., 2009; Verhage et al., 2010; Pieterse et al., 2012; Stam et al., 2014). Lipoxygenases (LOXs), a multi-gene family, are known to be involved in several developmental and defence-related biological processes such as fruit ripening, tuber development, seed germination and JA-regulated plant defences (Kolomiets et al., 2001; Bailly et al., 2002; Feussner \& Wasternack, 2002; Kessler, 2004; Barry \& Giovannoni, 2007; Yan et al., 2013). Upon feeding by many insect herbivores, LOXs catalyse oxygenation of polyunsaturated fatty acids (PUFAs) to initiate the formation of hydro-peroxides such as oxylipins (Shibata \& Axelrod, 1995; Brash, 1999; Feussner \& Wasternack, 2002). Several forms of oxylipins such as jasmonates, green leaf volatiles (GLVs) and death acids, are known to be involved in 
defence mechanisms against insect herbivory (Bell et al., 1995; Allmann et al., 2010; Yan et al., 2013; Shen et al., 2014; Christensen et al., 2015; Losvik et al., 2017). Furthermore, based on positional specificity to catalyse oxygenation of linoleic acids (LAs), LOXs are broadly classified into two major groups, 9- and 13-LOXs (Feussner \& Wasternack, 2002). Jasmonates and GLVs, involved in direct and indirect defences, are derived from 13-LOXs, whereas, death acids are derived from 9-LOXs. Furthermore, different numbers of LOXs are reported in different plant species such as Arabidopsis (6) (Umate, 2011), tomato (7), kiwifruit (6) (Zhang et al., 2006), olive (4) (Padilla et al., 2009, 2012), melon (18) (Zhang et al., 2014), cucumber (23) (Liu et al., 2011) and grapevine (18) (Podolyan et al., 2010). Among the LOXs in each plant species, one $L O X$ is involved in JA-biosynthesis induced upon insect herbivory or mechanical wounding. For example, AtLOX2 in Arabidopsis (Bell et al., 1995), SILOXD (TomLOXD) in tomato (Yan et al., 2013), NaLOX3 in tobacco (Halitschke \& Baldwin, 2003; Kessler, 2004) and StLOXH3 in potato (Royo et al., 1996) are known to code for enzymes involved in JA biosynthesis. Therefore, to understand the mechanism of JA-regulated defences, identifying the LOX involved in JA pathway is important.

\section{Thrip pests}

Thrips (Paraneoptera: Thysanoptera), are pest insects on many commercial and ornamental plants worldwide. The order Thysanoptera represents over 5500 species. Thrips are tiny (ca $1.5 \mathrm{~mm}$ ) cell-content feeding insects exerting direct damage to plants by inserting their stylets into plant tissue and ingesting the cell contents. The pierced tissue turns into silvery scars (Fig. 1C) hindering photosynthetic ability, growth, reproduction and yield of plants (Steiner, 1990; Welter et al., 1990; Shipp et al., 1998; Steenbergen et al., 2018). The success of thrips as a pest can be attributed to several of its characteristics, such as its short life-cycle, high polyphagy, thigmokinetic behaviour, high reproductive rate, and rapid adaptation to insecticides (Diaz-Montano et al., 2011; Gill et al., 2015; Steenbergen et al., 2018).

Besides inflicting direct damage, thrips also cause indirect damage by serving as vector to different tospoviruses. For example, western flower thrips (WFT; Frankliniella occidentalis) is known to transfer Tomato spotted wilt virus (TSWV) (Maris et al., 2003), whereas onion thrips (Thrips tabacl) is known to transfer Iris yellow spot virus (IYSV) (Bunyaviridae) (Diaz-Montano et al., 2011; Gill et al., 2015). Moreover, although western flower thrips is reported to be one of the economically most damaging species, several other thrips species, such as onion thrips, avocado thrips (Scitothrips perseae), blossom thrips (Frankliniella schultzei) and melon thrips (Thrips palmi) also contribute significantly to overall economic losses. The estimation of losses caused only by thrips feeding is hard, but the crop losses caused due to combined effect of thrips feeding and transferring the viral disease is huge. At present, thrips are mostly 
controlled by using pesticides. Therefore, to develop thrips-resistant crop varieties to minimize the damage, exploration and understanding of the genetic basis underlying plant defence responses is important. Such crop varieties may be a valuable component of integrated pest management, in combination with biological control (Mouden et al., 2017).
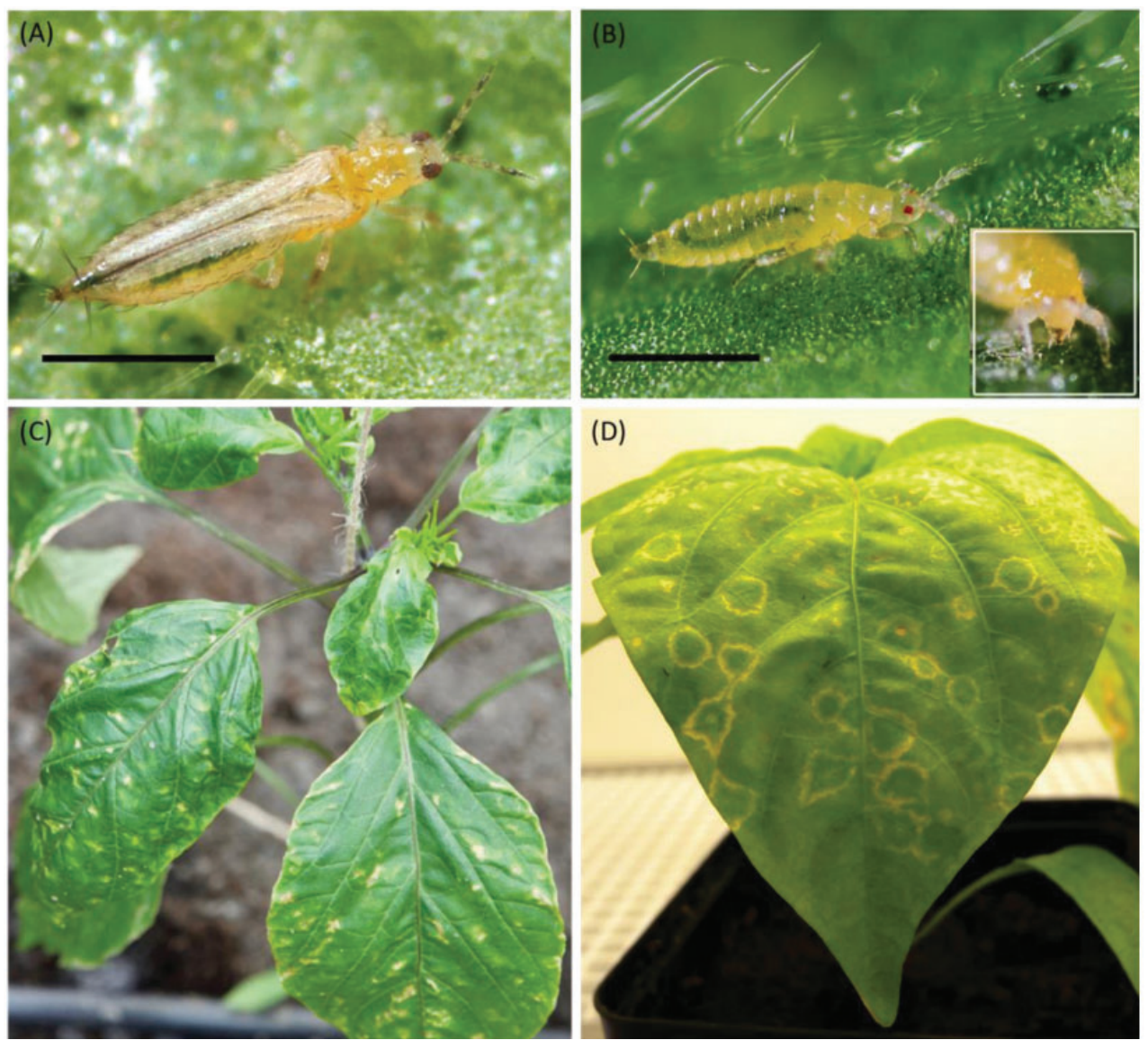

Figure 1. Different developmental stages of thrips and their inflicted damage on pepper plants. (A) Female adult Frankliniella occidentalis (Western flower thrips (WFT)), (B) second larval instar (L2) of WFT, (C) Silver scars resulting from feeding by thrips, (D) Symptoms of Tomato spotted wilt virus (TSWV). Adapted from Steenbergen et al. (2018).

\section{Thrips: Feeding behaviour and virus transmission}

\section{Feeding behaviour}

For a thorough understanding of how thrips damage plants, it is important to investigate their feeding behaviour in detail. Thrips feed on almost all aboveground parts of plants: leaves, flowers, fruits and stems. While feeding, they insert their stylets into 
the tissue of a plant and ingest cell contents (Hunter \& Ullman, 1992; Mound, 2005). During individual probes they can ingest cell contents from multiple cells (Kindt et al., 2003). Probing comprises of three distinct behavioural phases: (1) piercing into plant parts (2) salivation and (3) ingestion of cell contents. When feeding on leaves, thrips can only access cell contents of epidermal, mesophyll and parenchymal cells, since their stylets are too short to reach vascular tissues.

Kindt et al. (2003) characterized feeding behaviour of $F$. occidentalis into six different phases: $P, Q, R, S, T$ and $U$ using the electrical penetration graph (EPG) technique (Kindt et al., 2003; Kindt et al., 2006). These phases captured all minor behavioural activities of thrips during feeding, where each phase is represented by one waveform. Waveform $P$ depicts piercing of the leaf surface by the mandibular stylets, waveform $Q$ represents maxillary stylet insertion and the beginning of salivation and waveform $R$ represents ingestion of the cell contents. Finally, waveforms $S$ and $U$ represent the mandibular action and waveform $T$ represents withdrawal of stylets from leaf. Since not all these waveforms occur during every probe event, Kindt et al. (2006) proposed to discriminate the waveforms into two major phases, i.e. "puncture phase" $(P, Q$, plus S) and "feeding phase" (R, T, plus U) (Kindt et al., 2003; Kindt et al., 2006). Thus, with EPG and histological studies, details of the exact feeding mechanism of thrips as mediated by its stylets within the plant tissues have been elucidated.

\section{Virus transmission by thrips}

Besides direct damage, thrips also cause damage to plants indirectly by transmitting plant viruses like Tospoviruses, among which TSWV, that have significant economic impact (Maris et al., 2003; Steenbergen et al., 2018). Tospoviruses represent the only plant-infecting genus in the family Bunyaviridae, infecting thousands of plant species (Whitfield et al., 2005). In order to gain broad knowledge on how thrips damage plants indirectly, understanding of acquisition and transmission of viruses is important.

Acquisition of tospoviruses by thrips is dependent on thrips developmental stage. Viruses are generally acquired by immature first (L1) and second (L2) larval instars of thrips while feeding on viruliferous plants. The efficiency of acquiring viruses is higher at first instars (L1) and decreases as they develop (Rotenberg et al., 2015). The acquired viruses then replicate in the host vector and are transmitted to healthy plants thereafter (De Assis et al., 2004). The transmission of TSWV can occur as fast as in a single non-ingesting probe. This transmission of TSWV occurs during the salivation phase, i.e. Q-waveform of thrips feeding (Kindt et al., 2003). The success rate of transmission of TSWV by F. occidentalis in a thrips-TSWV susceptible variety was reported to be $0.8 \%$, i.e. one in 125 probes resulted in successful transmission of virus (Kindt, 2004). The probability of virus transmission by a viruliferous thrips increases with increasing numbers of probes. Likewise, the efficiency of transmission 
of viruses largely depends on the plant variety the thrips are feeding on. For example, virus inoculation efficiency was significantly lower on a thrips-resistant pepper accession during longer inoculation periods but no effect was seen in shorter inoculation periods (Maris et al., 2003). This indicates that varieties resistant to thrips can provide opportunities to control virus spread as well.

\section{Host-plant resistance to thrips}

Host-plant resistance to insects is an important component of integrated pest management (Sharma \& Ortiz, 2002). Understanding the mechanisms underlying genetic variation in resistance within a population of accessions can help to develop hostplant resistance against insects in a crop (Broekgaarden et al., 2011a). However, linking genetic variation to specific traits is a complex task, due to the involvement of the number of traits underlying plant-insect interactions. Host-plant resistance is regulated by a complex network of genes. As a consequence, the development of a reproducible high-throughput method providing information on different components of host-plant resistance is important (Kloth et al., 2012). At present, there are two major methods to determine host-plant resistance to thrips: (1) end-point assays, and (2) detailed observations of insect behaviour. In end-point assays, thrips performance is measured based on damage, mortality and reproduction on the host-plant, whereas, in behavioural assays, insect preference during the period of an experiment is recorded (Thoen et al., 2016). In end-point assays, host-plant resistance to thrips has been established from days to weeks post-inoculation. For this type of assays, thrips damage is assessed manually or automatically by using imaging tools. Additionally, other parameters of insect performance like survival, reproduction and mortality have also been recorded manually (Abe et al., 2008; Abe et al., 2009; Maharijaya et al., 2011; Leiss et al., 2013). Such assays are labour intensive and time consuming. Thoen et al. (2016) developed a high-throughput phenotyping platform that can screen hostplant resistance against $F$. occidentalis, where they considered thrips behaviour as a proxy of resistance. The method consists of multiple, simultaneous two-choice setups using computerized continuous video-tracking of thrips behaviour throughout a period of several hours. In this method, detailed behavioural parameters like time spent on either accession, movement (searching), non-movement (feeding) are recorded and analysed. With a higher time and resource efficiency, this method complements the results produced in end-point assays (Thoen et al., 2016). Although these two approaches produce quite different information on host-plant resistance against thrips, combining these methods can be used to efficiently find the factors responsible for host-plant resistance against thrips. The automated video-tracking method has as major advantage that hundred plants can be screened simultaneously per day. Thus, this method can be used to screen hundreds of plant samples for narrowing the selection, whereas end-point assays can be used to validate the selection made by the 
video-tracking method. Recently, the video-tracking system is updated to automation (known as T-maze arrays) making it less laborious and time-consuming (Jongsma et al., 2019). Therefore, this method can be a reliable and effective high-throughput phenotyping alternative to the behavioural assays.

\section{Induced plant defences upon thrips feeding}

Upon thrips feeding, plants trigger signal transduction pathways that regulate transcriptomic responses and biosynthesis of several processes, such as metabolites and defence-related proteins. The plant hormone JA is identified as a pivotal hormone in regulating induced defence against thrips (Fig. 2) (De Vos et al., 2005; Abe et al., 2008; Abe et al., 2009). In plants, such as, Arabidopsis and Chinese cabbage (Brassica rapa subsp. pekinensis), WFT feeding elevated the expression of JA-biosynthetic genes and JA hormonal levels (Abe et al., 2008; Abe et al., 2009). Defenceless1 (Def1), a JA-deficient tomato mutant, has reduced resistance to thrips feeding (Li et al., 2002; Escobar-Bravo et al., 2017). A full-genome microarray-based study in Arabidopsis showed that $69 \%$ of all differentially expressed genes (DEGs) upon thrips feeding were JA responsive (De Vos et al., 2005). Exogenous application of JA to several plants, such as Arabidopsis, Chinese cabbage (Brassica rapa subsp. pekinensis), tomato, soybean and cotton, enhanced the resistance against thrips (Omer et al., 2001; Thaler et al., 2001; Li et al., 2002; Abe et al., 2008; Abe et al., 2009; Selig et al., 2016; Escobar-Bravo et al., 2017).

Furthermore, plants produce defensive metabolites to defend themselves against herbivores. In response to thrips feeding, phenolic compounds are found to be produced by plants (Papadaki et al., 2008; Leiss et al., 2009; War et al., 2012). For instance, during pepper (Capsicum annuum) - F. occidentalis and alfalfa (Medicago sativa) Odontothrips loti interactions, levels of phenolic compounds like tocopherols and tannins were elevated, respectively, in resistant varieties of both plant species (Maris et al., 2003; Wang et al., 2014). In contrast, in pepper, thrips-induced metabolites such as alkanes and fatty acids correlated positively with susceptibility of pepper plants (Maharijaya et al., 2012). Furthermore, few studies have shown plants to modify their blend of volatile organic compounds (VOCs) in response to thrips infestation and attract natural enemies of thrips, thus activating their indirect defences. For instance, upon WFT feeding, cucumber plants attracted the predatory mite $N$. cucumeris and predatory bug Orius lavigatus (Venzon et al., 1999). Similarly, during eggplant - $T$. palmi and chrysanthemum (C. morifolium) - F. occidentalis interactions, infested eggplant (Solanum melongena) and chrysanthemum plants attracted Orius sauteri and Neoseiulus cucumeris, respectively (Manjunatha et al., 1998; Maris et al., 2003). 


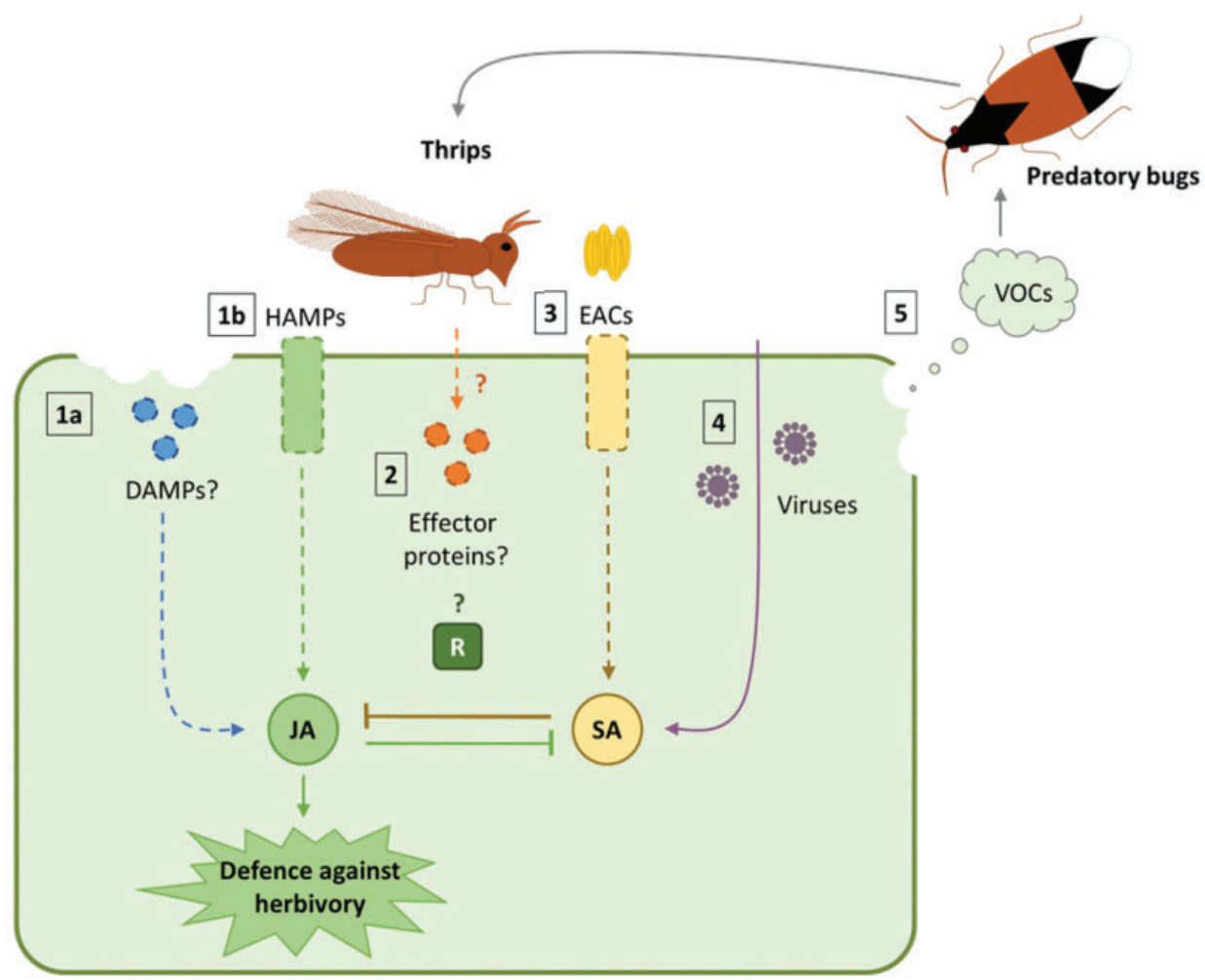

Figure 2. Proposed model of induced plant responses upon thrips feeding. During the plant-thrips interaction, plants initiate defence responses upon perceiving damage-associated molecular patterns (DAMPs) (1a) or herbivore-associated molecular patterns (HAMPs) (1b). This results into activation of JA, which further regulates the defences against thrips. Effector proteins (2) and egg associated compounds (EACs) (3) derived from thrips can manipulate induced plant defences in favour of thrips. Tospoviruses transmitted by thrips can alter plant defence by inducing SA, which in turn can supress JA-regulated responses (4) through crosstalk. Thrips feeding results in the release of a blend of VOCs that attracts natural enemies of thrips such as, Orius laevigatus (5). Dashed lines represent unidentified elements in induced responses against thrips. Adapted from Steenbergen et al. (2018).

\section{Research objective}

The objective of this thesis was to study the whole-genome transcriptional response of plants to thrips through a high-resolution temporal analysis and to investigate the genetic mechanisms activated in crop plants (sweet pepper and white cabbage) in response to thrips feeding. This includes identifying defence-related gene families, disentangling functions of genes and elucidating temporal whole-genome transcriptional response of sweet pepper and white cabbage against WFT and onion thrips feeding, respectively. 


\section{Study system}

\section{Plant species}

\section{Sweet pepper}

Sweet pepper (Capsicum annuum) [(Mandy variety, Rijk Zwaan (De Lier, The Netherlands))] plants (Fig. 3A) were used to study the transcriptional response to western flower thrips feeding. Sweet pepper is a diploid and self-pollinating crop belonging to the Solanaceae family. This family encompasses several other commercially important crops such as eggplant, tomato, tobacco, potato and petunia. Pepper has a high nutritional quality and provides us with important minerals, vitamins and nutrients. It can be produced for several purposes such as, in pharmaceuticals, organic colour, cosmetics and defence repellents. However, in spite of the rising economic importance of pepper, information on the underlying molecular mechanisms against herbivorous insects including thrips is limited.
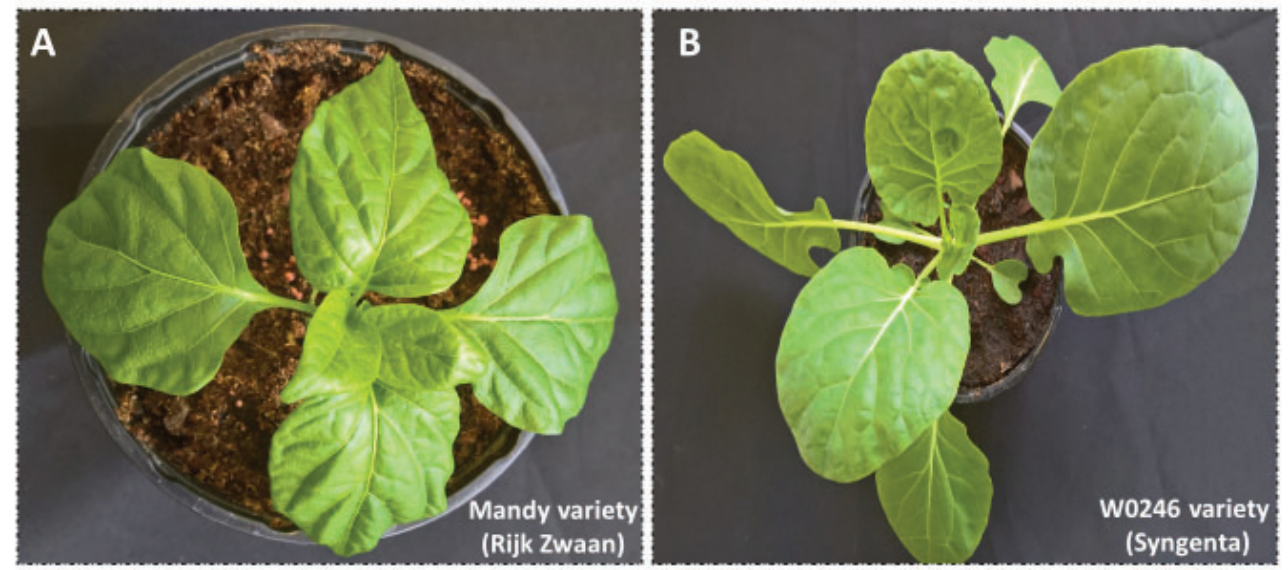

Figure 3. Sweet pepper and white cabbage plant varieties used in this thesis. (A) Sweet pepper (Mandy) and, (B) White cabbage (W0246) variety.

\section{White-cabbage}

White cabbage [Brassica oleracea (W0246 variety, Syngenta (Enkhuizen, The Netherlands))] plants (Fig. 3B) were used to investigate the temporal transcriptional response against onion thrips. White cabbage is an biennial plant grown as an annual, belonging to the Brassicaceae (Crucifer) family. Brassica oleracea comprises of several common food crops such as kale, cabbage, broccoli, cauliflower, Brussels sprouts and kohlrabi. The molecular mechanisms of white cabbage against insect herbivores have received limited attention. 


\section{Thrips species}

\section{Western flower thrips}

Western flower thrips (Frankliniella occidentalis) (Fig. 4A) were used to infest sweet pepper plants (Chapters 1, 2 and 3 of this thesis). In The Netherlands, WFT is a major pest on sweet pepper plants in greenhouses. Thrips are cell-content feeding generalist insects affecting plant productivity and yield by inflicting feeding damage and transferring tospoviruses such as, TSWV. In several plants, such as Arabidopsis, Chinese cabbage (Brassica rapa subsp. pekinensis) and tomato, they are known to elicit JA-regulated defences (Abe et al., 2008; Abe et al., 2009).
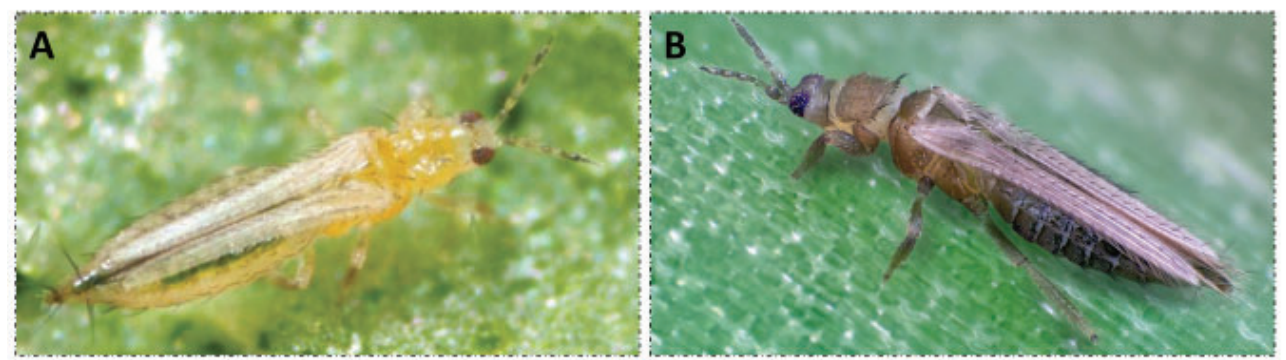

Figure 4. Thrips species used in this thesis. (A) Western flower thrips (Frankliniella occidentalis) interaction with sweet pepper and, (B) Onion thrips (Thrips tabaci) interaction with white cabbage. Photo credits: A modified from Steenbergen et al. (2018) and B adapted from Thrips-iD website: http://www.thrips-id.com/en/.

\section{Onion thrips}

Onion thrips, Thrips tabaci Lindeman (Thysanoptera: Thripidae) (Fig. 4B), was used in combination with white cabbage plants in Chapter 4 of this thesis. This thrips is a serious pest on white cabbage (B. oleracea) plants worldwide (Shelton et al., 2008; Fail et al., 2013). It has similar life-history characteristics as WFT, making them difficult to control. Onion thrips transmits other tospoviruses than WFT, i.e. Iris yellow spot virus (IYSV) (Bunyaviridae) (Diaz-Montano et al., 2011; Gill et al., 2015). To the best of my knowledge, no molecular study has been made yet in any plant on the response to onion thrips. 


\section{Outline of the thesis}

Chapter 2 focusses on the identification and classification of the lipoxygenase gene family in pepper. Implementing several approaches such as comparative genomics, domain-scan analysis, sequence analysis, phylogenetic analysis, homology modelling and transcriptional analysis, the lipoxygenase gene family of pepper (Capsicum annuum) was identified.

Chapter 3 focusses on the involvement of one LOX gene, i.e. CaLOX2, in jasmonate-dependent induced defence against western flower thrips in sweet pepper. With Virus-Induced Gene Silencing (VIGS) of CaLOX2, followed by several bioassays, the role of CaLOX2 in sweet pepper defences has been experimentally validated.

Chapter 4 focuses on the temporal whole-genome transcriptional response of sweet pepper plants in response to western flower thrips herbivory. A high-resolution RNASeq approach was used to unravel the temporal and chronological response of several hormonal, and secondary metabolite pathways. I also participated in a similar study to unravel temporal transcriptomic response of Arabidopsis during its interaction with WFT (Steenbergen et al., 2019). A Bi-directional Blast Homologue (BBH) approach was implemented to investigate commonalities and specifics in sweet pepper and Arabidopsis transcriptional responses, both induced upon WFT feeding.

Chapter 5 presents the temporal high-density transcriptional response of white cabbage plants in response to onion thrips herbivory and a comparative analysis on induced defences (transcriptomics) between WFT-induced Arabidopsis and sweet pepper and onion-thrips-induced white cabbage.

Finally, Chapter 6 integrates the key results of this thesis and discusses in the context of the current knowledge of dynamics of plant transcriptional responses to different stresses.

\section{Acknowledgements}

I express my gratitude to Marcel Dicke for his constructive comments on an earlier version of this chapter and to Merel Steenbergen for kindly providing figures 1, 2 and 4A. This work is part of STW perspective programme Green Defences Against Pests (GAP) that is (partly) financed by the Netherlands Organisation for Scientific Research (NWO, grant no. 13555). 


\section{References}

Abe H, Ohnishi J, Narusaka M, Seo S, Narusaka Y, Tsuda S, Kobayashi M. 2008. Function of jasmonate in response and tolerance of Arabidopsis to thrip feeding. Plant Cell Physiology 49(1): 68-80.

Abe H, Shimoda T, Ohnishi J, Kugimiya S, Narusaka M, Seo S, Narusaka Y, Tsuda S, Kobayashi M. 2009. Jasmonate-dependent plant defense restricts thrips performance and preference. BMC Plant Biology 9: 97.

Allmann S, Halitschke R, Schuurink RC, Baldwin IT. 2010. Oxylipin channelling in Nicotiana attenuata: lipoxygenase 2 supplies substrates for green leaf volatile production. Plant Cell and Environment 33(12): 2028-2040.

Appel HM, Maqbool SB, Raina S, Jagadeeswaran G, Acharya BR, Hanley JC, Miller KP, Hearnes L, Jones AD, Raina R, Schultz JC. 2014. Transcriptional and metabolic signatures of Arabidopsis responses to chewing damage by an insect herbivore and bacterial infection and the consequences of their interaction. Frontiers in Plant Science 5: 441.

Bailly C, Bogatek-Leszczynska R, Come D, Corbineau F. 2002. Changes in activities of antioxidant enzymes and lipoxygenase during growth of sunflower seedlings from seeds of different vigour. Seed Science Research 12(1): 47-55.

Barry CS, Giovannoni JJ. 2007. Ethylene and fruit ripening. Journal of Plant Growth Regulation 26(2): 143-159.

Bell E, Creelman RA, Mullet JE. 1995. A chloroplast lipoxygenase is required for wound-induced jasmonic acid accumulation in Arabidopsis. Proceedings of the National Academy of Sciences of the United States of America 92(19): 8675-8679.

Bidart-Bouzat MG, Kliebenstein D. 2011. An ecological genomic approach challenging the paradigm of differential plant responses to specialist versus generalist insect herbivores. Oecologia 167(3): 677-689.

Brash AR. 1999. Lipoxygenases: Occurrence, functions, catalysis, and acquisition of substrate. Journal of Biological Chemistry 274(34): 23679-23682.

Breeze E, Harrison E, McHattie S, Hughes L, Hickman R, Hill C, Kiddle S, Kim YS, Penfold CA, Jenkins D, Zhang CJ, Morris K, Jenner C, Jackson S, Thomas B, Tabrett A, Legaie R, Moore JD, Wild DL, Ott S, Rand D, Beynon J, Denby K, Mead A, Buchanan-Wollaston V. 2011. High-resolution temporal profiling of transcripts during Arabidopsis leaf senescence reveals a distinct chronology of processes and regulation. Plant Cell 23(3): 873-894.

Broekgaarden C, Pelgrom KTB, Bucher J, van Dam NM, Grosser K, Pieterse CMJ, van Kaauwen M, Steenhuis G, Voorrips RE, de Vos M, Vosman B, Worrich A, van Wees SCM. 2018. Combining QTL mapping with transcriptome and metabolome profiling reveals a possible role for ABA signaling in resistance against the cabbage whitefly in cabbage. PLoS One 13(11): e0206103.

Broekgaarden C, Snoeren TAL, Dicke M, Vosman B. 2011a. Exploiting natural variation to identify insect-resistance genes. Plant Biotechnology Journal 9(8): 819-825.

Broekgaarden C, Voorrips RE, Dicke M, Vosman B. 2011b. Transcriptional responses of Brassica nigra to feeding by specialist insects of different feeding guilds. Insect Science 18(3): 259-272.

Bui H, Greenhalgh R, Ruckert A, Gill GS, Lee S, Ramirez RA, Clark RM. 2018. Generalist and specialist mite herbivores induce similar defense responses in maize and barley but differ in susceptibility to benzoxazinoids. Frontiers in Plant Science 9: 1222. 
Christensen SA, Huffaker A, Kaplan F, Sims J, Ziemann S, Doehlemann G, Ji L, Schmitz RJ, Kolomiets MV, Alborn HT, Mori N, Jander G, Ni X, Sartor RC, Byers S, Abdo Z, Schmelz EA. 2015. Maize death acids, 9-lipoxygenase-derived cyclopente(a)nones, display activity as cytotoxic phytoalexins and transcriptional mediators. Proceedings of the National Academy of Sciences of the United States of America 112(36): 11407-11412.

Coolen S, Proietti S, Hickman R, Davila Olivas NH, Huang PP, Van Verk MC, Van Pelt JA, Wittenberg AHJ, De Vos M, Prins M, Van Loon JJA, Aarts MGM, Dicke M, Pieterse CMJ, Van Wees SCM. 2016. Transcriptome dynamics of Arabidopsis during sequential biotic and abiotic stresses. Plant Journal 86(3): 249-267.

Davila Olivas NH, Coolen S, Huang PP, Severing E, van Verk MC, Hickman R, Wittenberg AHJ, de Vos M, Prins M, van Loon JJA, Aarts MGM, van Wees SCM, Pieterse CMJ, Dicke M. 2016. Effect of prior drought and pathogen stress on Arabidopsis transcriptome changes to caterpillar herbivory. New Phytologist 210(4): 1344-1356.

De Assis FM, Deom CA, Sherwood JL. 2004. Acquisition of Tomato spotted wilt virus by adults of two thrips species. Phytopathology 94(4): 333-336.

De Vos M, Van Oosten VR, Van Poecke RMP, Van Pelt JA, Pozo MJ, Mueller MJ, Buchala AJ, Metraux JP, Van Loon LC, Dicke M, Pieterse CMJ. 2005. Signal signature and transcriptome changes of Arabidopsis during pathogen and insect attack. Molecular Plant-Microbe Interactions 18(9): 923-937.

Diaz-Montano J, Fuchs M, Nault BA, Fail J, Shelton AM. 2011. Onion thrips (Thysanoptera: Thripidae): A global pest of increasing concern in onion. Journal of Economic Entomology 104(1): 1-13.

Diaz-Riquelme J, Zhurov V, Rioja C, Perez-Moreno I, Torres-Perez R, Grimplet J, Carbonell-Bejerano P, Bajda S, Van Leeuwen T, Martinez-Zapater JM, Grbic M, Grbic V. 2016. Comparative genome-wide transcriptome analysis of Vitis vinifera responses to adapted and non-adapted strains of two-spotted spider mite, Tetranyhus urticae. BMC Genomics 17: 74.

Dicke M. 2009. Behavioural and community ecology of plants that cry for help. Plant Cell and Environment 32(6): 654-665.

Dicke M. 2015. Herbivore-induced plant volatiles as a rich source of information for arthropod predators: Fundamental and applied aspects. Journal of the Indian Institute of Science 95(1): 35-42.

Douglas AE. 2018. Strategies for enhanced crop resistance to insect pests. Annual Review of Plant Biology 69: 637-660.

Duran-Flores D, Heil M. 2016. Sources of specificity in plant damaged-self recognition. Current Opinion in Plant Biology 32: 77-87.

Durrant M, Boyer J, Zhou WW, Baldwin IT, Xu SQ. 2017. Evidence of an evolutionary hourglass pattern in herbivory-induced transcriptomic responses. New Phytologist 215(3): 1264-1273.

Ehlting J, Chowrira SG, Mattheus N, Aeschliman DS, Arimura GI, Bohlmann J. 2008. Comparative transcriptome analysis of Arabidopsis thaliana infested by diamond back moth (Plutella xylostella) larvae reveals signatures of stress response, secondary metabolism, and signalling. BMC Genomics 9: 154.

Escobar-Bravo R, Klinkhamer PGL, Leiss KA. 2017. Induction of jasmonic acid-associated defenses by thrips alters host suitability for conspecifics and correlates with increased trichome densities in Tomato. Plant and Cell Physiology 58(3): 622-634.

Fail J, Deutschlander ME, Shelton AM. 2013. Antixenotic resistance of cabbage to onion 
thrips (Thysanoptera: Thripidae). I. light reflectance. Journal of Economic Entomology 106(6): 2602-2612.

Feussner I, Wasternack C. 2002. The lipoxygenase pathway. Annual Review of Plant Biology 53: 275-297.

Gill HK, Garg H, Gill AK, Gillett-Kaufman JL, Nault BA. 2015. Onion thrips (Thysanoptera: Thripidae) biology,ecology, and management in onion production systems. Journal of Integrated Pest Management 6(1): 6.

Giron D, Huguet E, Stone GN, Body M. 2016. Insect-induced effects on plants and possible effectors used by galling and leaf-mining insects to manipulate their host-plant. Journal of Insect Physiology 84: 70-89.

Halitschke R, Baldwin IT. 2003. Antisense LOX expression increases herbivore performance by decreasing defense responses and inhibiting growth-related transcriptional reorganization in Nicotiana attenuata. Plant Journal 36(6): 794-807.

Heidel-Fischer HM, Musser RO, Vogel H 2014. Plant transcriptomic responses to herbivory. Annual Plant Reviews: John Wiley \& Sons, Ltd, 155-196.

Hickman R, Van Verk MC, Van Dijken AJH, Mendes MP, Vroegop-Vos IA, Caarls L, Steenbergen M, Van der Nagel I, Wesselink GJ, Jironkin A, Talbot A, Rhodes J, De Vries M, Schuurink RC, Denby K, Pieterse CMJ, Wees SCM. 2017. Architecture and dynamics of the jasmonic acid gene regulatory network. Plant Cell 29(9): 2086-2105.

Hilker M, Fatouros NE. 2016. Resisting the onset of herbivore attack: plants perceive and respond to insect eggs. Current Opinion in Plant Biology 32: 9-16.

Hogenhout SA, Bos JIB. 2011. Effector proteins that modulate plant-insect interactions. Current Opinion in Plant Biology 14(4): 422-428.

Howe GA, Jander G. 2008. Plant immunity to insect herbivores. Annual Review of Plant Biology 59: 41-66.

Hunter WB, Ullman DE. 1992. Anatomy and ultrastructure of the piercing-sucking mouthparts and paraglossal sensilla of Frankliniella occidentalis (Pergande) (Thysanoptera, Thripidae). International Journal of Insect Morphology \& Embryology 21(1): 17-35.

Jongsma MA, Thoen MPM, Poleij LM, Wiegers GL, Goedhart PW, Dicke M, Noldus LPJJ, Kruisselbrink JW. 2019. An integrated system for the automated recording and analysis of insect behavior in T-maze arrays. Frontiers in Plant Science 10: 20.

Kant MR, Jonckheere W, Knegt B, Lemos F, Liu J, Schimmel BCJ, Villarroel CA, Ataide LMS, Dermauw W, Glas JJ, Egas M, Janssen A, Van Leeuwen T, Schuurink RC, Sabelis MW, Alba JM. 2015. Mechanisms and ecological consequences of plant defence induction and suppression in herbivore communities. Annals of Botany 115(7): 1015-1051.

Kempema LA, Cui X, Holzer FM, Walling LL. 2007. Arabidopsis transcriptome changes in response to phloem-feeding silverleaf whitefly nymphs. Similarities and distinctions in responses to aphids. Plant Physiology 143(2): 849-865.

Kessler A. 2004. Silencing the jasmonate cascade: Induced plant defenses and insect populations. Science 306(5704): 2042-2042.

Kindt F. 2004. Probing behaviour of thrips: PhD thesis. Wageningen, The Netherlands: Wageningen University.

Kindt F, Joosten NN, Peters D, Tjallingii WF. 2003. Characterisation of the feeding behaviour of western flower thrips in terms of electrical penetration graph (EPG) waveforms. Journal of Insect Physiology 49(3): 183-191. 
Kindt F, Joosten NN, Tjallingii WF. 2006. Electrical penetration graphs of thrips revised: Combining DC- and AC-EPG signals. Journal of Insect Physiology 52(1): 1-10.

Kloth KJ, Thoen MPM, Bouwmeester HJ, Jongsma MA, Dicke M. 2012. Association mapping of plant resistance to insects. Trends in Plant Science 17(5): 311-319.

Kolomiets MV, Hannapel DJ, Chen H, Tymeson M, Gladon RJ. 2001. Lipoxygenase is involved in the control of potato tuber development. Plant Cell 13(3): 613-626.

Kroes A, Broekgaarden C, Uribe MC, May S, van Loon JJA, Dicke M. 2017. Brevicoryne brassicae aphids interfere with transcriptome responses of Arabidopsis thaliana to feeding by Plutella xylostella caterpillars in a density-dependent manner. Oecologia 183(1): 107-120.

Labandeira C. 2007. The origin of herbivory on land: Initial patterns of plant tissue consumption by arthropods. Insect Science 14(4): 259-275.

Leiss KA, Cristofori G, Van Steenis R, Verpoorte R, Klinkhamer PGL. 2013. An eco-metabolomic study of host plant resistance to Western flower thrips in cultivated, biofortified and wild carrots. Phytochemistry 93: 63-70.

Leiss KA, Maltese F, Choi YH, Verpoorte R, Klinkhamer PGL. 2009. Identification of chlorogenic acid as a resistance factor for thrips in chrysanthemum. Plant Physiology 150(3): 1567-1575.

Li CY, Williams MM, Loh YT, Lee GI, Howe GA. 2002. Resistance of cultivated tomato to cell content-feeding herbivores is regulated by the octadecanoid-signaling pathway. Plant Physiology 130(1): 494-503.

Liu SQ, Liu XH, Jiang LW. 2011. Genome-wide identification, phylogeny and expression analysis of the lipoxygenase gene family in cucumber. Genetics and Molecular Research 10(4): 2613-2636.

Losvik A, Beste L, Glinwood R, Ivarson E, Stephens J, Zhu LH, Jonsson L. 2017. Overexpression and down-regulation of barley lipoxygenase LOX2.2 affects jasmonate-regulated genes and aphid fecundity. International Journal of Molecular Sciences 18(12): 2765; doi:2710.3390/ijms18122765.

Maffei ME, Mithofer A, Boland W. 2007. Before gene expression: early events in plant-insect interaction. Trends in Plant Science 12(7): 310-316.

Maharijaya A, Vosman B, Steenhuis-Broers G, Harpenas A, Purwito A, Visser RGF, Voorrips RE. 2011. Screening of pepper accessions for resistance against two thrips species (Frankliniella occidentalis and Thrips parvispinus). Euphytica 177(3): 401-410.

Maharijaya A, Vosman B, Verstappen F, Steenhuis-Broers G, Mumm R, Purwito A, Visser RGF, Voorrips RE. 2012. Resistance factors in pepper inhibit larval development of thrips (Frankliniella occidentalis). Entomologia Experimentalis Et Applicata 145(1): 62-71.

Manjunatha M, Pickett J, Wadhams L, Nazzi F. 1998. Response of western flower thrips, Frankliniella occidentalis and its predator Amblyseius cucumeris to chrysanthemum volatiles in olfactometer and greenhouse trials. Insect Science and Its Application 18(2): 139-144.

Maris PC, Joosten NN, Peters D, Goldbach RW. 2003. Thrips resistance in pepper and its consequences for the acquisition and inoculation of Tomato spotted wilt virus by the western flower thrips. Phytopathology 93(1): 96-101.

Martel C, Zhurov V, Navarro M, Martinez M, Cazaux M, Auger P, Migeon A, Santamaria ME, Wybouw N, Diaz I, Van Leeuwen T, Navajas M, Grbic M, Grbic V. 2015. Tomato whole genome transcriptional response to Tetranychus urticae identifies divergence of spider mite-induced responses between tomato and Arabidopsis. Molecular Plant-Mi- 
crobe Interactions 28(3): 343-361.

McCormick AC, Unsicker SB, Gershenzon J. 2012. The specificity of herbivore-induced plant volatiles in attracting herbivore enemies. Trends in Plant Science 17(5): 303-310.

Mithofer A, Boland W. 2008. Recognition of herbivory-associated molecular patterns. Plant Physiology 146(3): 825-831.

Mithofer A, Boland W. 2012. Plant defense against herbivores: Chemical aspects. Annual Review of Plant Biology 63: 431-450.

Mouden S, Sarmiento KF, Klinkhamer PGL, Leiss KA. 2017. Integrated pest management in western flower thrips: past, present and future. Pest Management Science 73(5): 813822.

Mound LA. 2005. Thysanoptera: Diversity and interactions. Annual Review of Entomology 50: 247-269.

Omer AD, Granett J, Karban R, Villa EM. 2001. Chemically-induced resistance against multiple pests in cotton. International Journal of Pest Management 47(1): 49-54.

Padilla MN, Hernandez ML, Sanz C, Martinez-Rivas JM. 2009. Functional characterization of two 13-lipoxygenase genes from olive fruit in relation to the biosynthesis of volatile compounds of virgin olive oil. Journal of Agricultural and Food Chemistry 57(19): 9097-9107.

Padilla MN, Hernandez ML, Sanz C, Martinez-Rivas JM. 2012. Molecular cloning, functional characterization and transcriptional regulation of a 9-lipoxygenase gene from olive. Phytochemistry 74 : 58-68.

Papadaki M, Harizanova V, Dagnon S. 2008. Influence of feeding of Frankliniella occidentalis Pergande (Thysanoptera:Thripidae) on the polyphenolic complex in the leaves. Bulgarian Journal of Agricultural Science 14(4): 405-409.

Pieterse CMJ, Leon-Reyes A, Van der Ent S, Van Wees SCM. 2009. Networking by small-molecule hormones in plant immunity. Nature Chemical Biology 5(5): 308-316.

Pieterse CMJ, Van der Does D, Zamioudis C, Leon-Reyes A, Van Wees SCM. 2012. Hormonal modulation of plant immunity. Annual Review of Cell and Developmental Biology 28: 489-521.

Podolyan A, White J, Jordan B, Winefield C. 2010. Identification of the lipoxygenase gene family from Vitis vinifera and biochemical characterisation of two 13-lipoxygenases expressed in grape berries of Sauvignon Blanc. Functional Plant Biology 37(8): 767-784.

Poelman EH, Van Loon JJA, Van Dam NM, Vet LEM, Dicke M. 2010. Herbivore-induced plant responses in Brassica oleracea prevail over effects of constitutive resistance and result in enhanced herbivore attack. Ecological Entomology 35(2): 240-247.

Reymond P. 2013. Perception, signaling and molecular basis of oviposition-mediated plant responses. Planta 238(2): 247-258.

Reymond P, Bodenhausen N, Van Poecke RM, Krishnamurthy V, Dicke M, Farmer EE. 2004. A conserved transcript pattern in response to a specialist and a generalist herbivore. Plant Cell 16(11): 3132-3147.

Rotenberg D, Jacobson AL, Schneweis DJ, Whiffleld AE. 2015. Thrips transmission of tospoviruses. Current Opinion in Virology 15: 80-89.

Royo J, Vancanneyt G, Perez AG, Sanz C, Stormann K, Rosahl S, SanchezSerrano JJ. 1996. Characterization of three potato lipoxygenases with distinct enzymatic activities and different organ-specific and wound-regulated expression patterns. Journal of Biological Chemistry 271(35): 21012-21019. 
Selig P, Keough S, Nalam VJ, Nachappa P. 2016. Jasmonate-dependent plant defenses mediate soybean thrips and soybean aphid performance on soybean. Arthropod-Plant Interactions 10(4): 273-282.

Sharma HC, Ortiz R. 2002. Host plant resistance to insects: An eco-friendly approach for pest management and environment conservation. Journal of Environmental Biology 23(2): 111-135.

Shelton AM, Plate J, Chen M. 2008. Advances in control of onion thrips (Thysanoptera : Thripidae) in cabbage. Journal of Economic Entomology 101(2): 438-443.

Shen JY, Tieman D, Jones JB, Taylor MG, Schmelz E, Huffaker A, Bies D, Chen KS, Klee HJ. 2014. A 13-lipoxygenase, TomloxC, is essential for synthesis of C5 flavour volatiles in tomato. Journal of Experimental Botany 65(2): 419-428.

Shibata D, Axelrod B. 1995. Plant lipoxygenases. Journal of Lipid Mediators and Cell Signalling 12(2-3): 213-228.

Shipp JL, Hao X, Papadopoulos AP, Binns MR. 1998. Impact of western flower thrips (Thysanoptera : Thripidae) on growth, photosynthesis and productivity of greenhouse sweet pepper. Scientia Horticulturae 72(2): 87-102.

Stam JM, Dicke M, Poelman EH. 2018. Order of herbivore arrival on wild cabbage populations influences subsequent arthropod community development. Oikos 127(10): 1482-1493.

Stam JM, Kroes A, Li YH, Gols R, van Loon JJA, Poelman EH, Dicke M. 2014. Plant interactions with multiple insect herbivores: From community to genes. Annual Review of Plant Biology 65: 689-713.

Steenbergen M, Abd-el-Haliem A, Bleeker P, Dicke M, Escobar-Bravo R, Cheng G, Haring MA, Kant MR, Kappers I, Klinkhamer PGL, Leiss KA, Legarrea S, Macel M, Mouden S, Pieterse CMJ, Sarde SJ, Schuurink RC, De Vos M, Van Wees SCM, Broekgaarden C. 2018. Thrips advisor: exploiting thrips-induced defences to combat pests on crops. Journal of Experimental Botany 69(8): 1837-1848.

Steenbergen M, Hickman R, Sarde S, Dicke M, Pieterse CMJ, Van Wees SCM. 2019. High-resolution temporal transcriptomic dynamics of Arabidopsis thaliana in response to Western flower thrips feeding. In prep.

Steiner MY. 1990. Determining population characteristics and sampling procedures for the western flower thrips (Thysanoptera, Thripidae) and the predatory mite Amblyseius cucumeris (Acari, Phytoseiidae) on greenhouse cucumber. Environmental Entomology 19(5): 1605-1613.

Thaler JS, Stout MJ, Karban R, Duffey SS. 2001. Jasmonate-mediated induced plant resistance affects a community of herbivores. Ecological Entomology 26(3): 312-324.

Thoen MPM, Kloth KJ, Wiegers GL, Krips OE, Noldus LPJJ, Dicke M, Jongsma MA. 2016. Automated video tracking of thrips behavior to assess host-plant resistance in multiple parallel two-choice setups. Plant Methods 12: 1.

Tu XB, Liu ZK, Zhang ZH. 2018. Comparative transcriptomic analysis of resistant and susceptible alfalfa cultivars (Medicago sativa L.) after thrips infestation. BMC Genomics 19: 10.1186/s12864-018-4495-2.

Tzin V, Fernandez-Pozo N, Richter A, Schmelz EA, Schoettner M, Schafer M, Ahern KR, Meihls LN, Kaur H, Huffaker A, Mori N, Degenhardt J, Mueller LA, Jander G. 2015. Dynamic maize responses to aphid feeding are revealed by a time series of transcriptomic and metabolomic assays. Plant Physiology 169(3): 1727-1743.

Umate P. 2011. Genome-wide analysis of lipoxygenase gene family in Arabidopsis and rice. Plant Signaling and Behavior 6(3): 335-338. 
Venzon M, Janssen A, Sabelis MW. 1999. Attraction of a generalist predator towards herbivore-infested plants. Entomologia Experimentalis Et Applicata 93(3): 305-314.

Verhage A, van Wees SCM, Pieterse CMJ. 2010. Plant immunity: It's the hormones talking, but what do they say? Plant Physiology 154(2): 536-540.

Walling LL. 2008. Avoiding effective defenses: Strategies employed by phloem-feeding insects. Plant Physiology 146(3): 859-866.

Wang XS, Yang CL, Wang SS, Hu GX. 2014. Changes of phenols and lignin contents in alfalfa leaf damaged by Odontothrips loti. Ying Yong Sheng Tai Xue Bao 25(6): 1688-1692.

War AR, Paulraj MG, Ahmad T, Buhroo AA, Hussain B, Ignacimuthu S, Sharma HC. 2012. Mechanisms of plant defense against insect herbivores. Plant Signaling and Behavior 7(10): 1306-1320.

Welter SC, Rosenheim JA, Johnson MW, Mau RFL, Gusukumaminuto LR. 1990. Effects of thrips-palmi and western flower thrips (Thysanoptera, Thripidae) on the yield, growth, and carbon allocation pattern in cucumbers. Journal of Economic Entomology 83(5): 20922101.

Whitfield AE, Ullman DE, German TL. 2005. Tospovirus-thrips interactions. Annual Review of Phytopathology 43: 459-489.

Windram O, Madhou P, McHattie S, Hill C, Hickman R, Cooke E, Jenkins DJ, Penfold CA, Baxter L, Breeze E, Kiddle SJ, Rhodes J, Atwell S, Kliebenstein DJ, Kim YS, Stegle O, Borgwardt K, Zhang CJ, Tabrett A, Legaie R, Moore J, Finkenstadt B, Wild DL, Mead A, Rand D, Beynon J, Ott S, Buchanan-Wollaston V, Denby KJ. 2012. Arabidopsis defense against Botrytis cinerea: Chronology and regulation deciphered by high-resolution temporal transcriptomic analysis. Plant Cell 24(9): 3530-3557.

Yan LH, Zhai QZ, Wei JN, Li SY, Wang B, Huang TT, Du MM, Sun JQ, Kang L, Li CB, Li CY. 2013. Role of Tomato lipoxygenase $D$ in wound-induced jasmonate biosynthesis and plant immunity to insect herbivores. PLoS Genetics 9(12): e1003964.

Zhang B, Chen KS, Bowen J, Allan A, Espley R, Karunairetnam S, Ferguson I. 2006. Differential expression within the LOX gene family in ripening kiwifruit. Journal of Experimental Botany 57(14): 3825-3836.

Zhang C, Jin YZ, Liu JY, Tang YF, Cao SX, Qi HY. 2014. The phylogeny and expression profiles of the lipoxygenase ( $L O X$ ) family genes in the melon (Cucumis melo L.) genome. Scientia Horticulturae 170: 94-102.

Zhang PJ, Broekgaarden C, Zheng SJ, Snoeren TAL, van Loon JJA, Gols R, Dicke M. 2013. Jasmonate and ethylene signaling mediate whitefly-induced interference with indirect plant defense in Arabidopsis thaliana. New Phytologist 197(4): 1291-1299.

Zhu-Salzman K, Salzman RA, Ahn JE, Koiwa H. 2004. Transcriptional regulation of sorghum defense determinants against a phloem-feeding aphid. Plant Physiology 134(1): 420-431. 



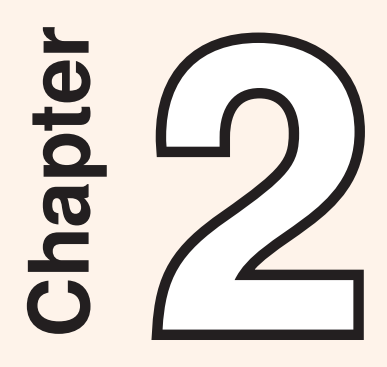

Genome-wide identification, classification and expression of lipoxygenase gene family in pepper

SJ. Sarde, A. Kumar, RN. Remme and M. Dicke

Published in Plant Molecular Biology (2018), doi:10.1007/s11103-018-0785-y 


\section{Abstract}

Lipoxygenases (LOXs) are non-heme, iron-containing dioxygenases playing a pivotal role in diverse biological processes in plants, including defence and development. Here, we exploited the recent sequencing of the pepper genome to investigate the LOX gene family in pepper. Two LOX classes are recognized, the 9- and 13-LOXs that oxygenate lipids at the 9th and 13th carbon atom, respectively. Using two main in-silico approaches, we identified a total of eight LOXs in pepper. Phylogenetic analysis classified four LOXs (CaLOX1, CaLOX3, CaLOX4 and CaLOX5) as 9-LOXs and four (CaLOX2, CaLOX6, CaLOX7 and CaLOX8) as 13-LOXs. Furthermore, sequence similarity/identity and subcellular localization analysis strengthen the classification predicted by phylogenetic analysis. Pivotal amino acids together with all domains and motifs are highly conserved in all pepper LOXs. Expression of 13-LOXs appeared to be more dynamic compared to 9- LOXs both in response to exogenous JA application and to thrips feeding. Bioinformatic and expression analyses predict the putative functions of two 13-LOXs, CaLOX6 and CaLOX7, in the biosynthesis of Green Leaf Volatiles, involved in indirect defence. The data are discussed in the context of LOX families in solanaceous plants and plants of other families.

Key words: Pepper, lipoxygenases (LOXs), phylogenetic analysis, gene transcription, sequence analyses, defence 


\section{Introduction}

Lipoxygenases (EC 1.13.11.12) are non-heme, iron-containing dioxygenases ubiquitously present in plants, animals and fungi (Brash, 1999). In plants, lipoxygenases (LOXs) are well-known to be involved in several plant processes like tuber development, seed germination, fruit ripening and most importantly in plant defences (Kolomiets et al., 2001; Bailly et al., 2002; Feussner \& Wasternack, 2002; Kessler, 2004; Barry \& Giovannoni, 2007; Yan et al., 2013). Upon insect or pathogen attack, LOXs oxidize polyunsaturated fatty acids (PUFAs) (linoleic acid, a-linolenic acid and arachidonic acid) constituting a (Z,Z)-1,4-pentadiene structural unit and catalyzing it into conjugated hydro-peroxides such as oxylipins (Shibata \& Axelrod, 1995; Brash, 1999; Feussner \& Wasternack, 2002). Oxylipins such as jasmonates, green leaf volatiles (GLVs) and recently discovered death acids, are known for their roles in defence against herbivorous insects and pathogens (Bell et al., 1995; Allmann et al., 2010; Yan et al., 2013; Shen et al., 2014; Christensen et al., 2015; Losvik et al., 2017). Jasmonates and GLVs are 13-LOX-derived products involved in direct and indirect defences, respectively. In indirect defence, GLVs play a pivotal role in the attraction of natural enemies of the herbivores (ul Hassan et al., 2015). Death acids (10-OPDA, 10-oxo-11-phytodienoic acid, and 10-OPEA, 10-oxo-11-phytoenoic acid) are 9-LOX-derived products that in maize (Zea mays) accumulate upon southern leaf blight (Cochliobolus heterostrophus) infection resulting in the hampering of growth of fungi and herbivorous insects (Christensen et al., 2015; Christensen et al., 2016).

Plant LOXs are primarily classified into two major classes, 9- and 13-LOXs, based on their positional specificity to oxygenate linoleic acids (LAs) (Feussner \& Wasternack, 2002). Moreover, LOXs are also classified as Type-1 and Type-2 based on their primary structure and sequence similarity. LOXs having high sequence similarity $(>75 \%)$ among themselves and having no plastidic transit peptide are classified as Type-1, whereas LOXs with moderate sequence similarity ( $>35 \%)$ and possessing a plastidic transit peptide are classified as Type-2 (Brash, 1999; Feussner \& Wasternack, 2002). All Type-2 LOXs known at present are 13-LOXs, whereas Type-1 LOXs include both 9- and 13-LOXs (Feussner \& Wasternack, 2002).

Information on LOXs from several plants has been reported. The Arabidopsis genome comprises a total of six LOXs (AtLOX1 - AtLOX6) (Umate, 2011). AtLOX1 is up-regulated in leaves upon pathogen attack and stress-related hormones (Melan et al., 1993); AtLOX2 is involved in jasmonic acid (JA) biosynthesis (Bell et al., 1995); AtLOX3 and AtLOX4 are essential for flower growth and male fertility (Caldelari et al., 2011); AtLOX5 is important for lateral root development and defence responses (Vellosillo et al., 2007) and AtLOX6 is expressed in roots and involved in JA synthesis 
(Grebner et al., 2013). Among solanaceous plants, different numbers of LOXs are reported in tomato, potato and tobacco. In tomato, SILOXA (TomLOXA) and SILOXB (TomLOXB) are induced during fruit ripening (Ferrie et al., 1994; Griffiths et al., 1999); SILOXC (TomLOXC) participates in production of flavour compounds resulting from fatty acids (Chen et al., 2004); SILOXD (TomLOXD) is involved in wound-induced JA biosynthesis, enhancing resistance against herbivores and pathogens (Yan et al., 2013); SILOXE (TOMLOXE) is expressed in breaker fruit (Chen et al., 2004) and SILOXF (TomLOXF) enhances systemic resistance stimulated by Pseudomonas putida BTP1(Mariutto et al., 2011). In tobacco, NaLOX1 codes for a 9-LOX and is specifically expressed in roots (Allmann et al., 2010); NaLOX2 is involved in biosynthesis of GLVs (Allmann et al., 2010; VanDoorn et al., 2010); and NaLOX3 is involved in JA biosynthesis (Halitschke \& Baldwin, 2003; Kessler, 2004). Furthermore, in potato, StLOXH1 mediates the biosynthesis of volatile C6-aldehydes (GLVs) involved in defence (Leon et al., 2002) and StLOXH3 is involved in the JA biosynthetic pathway (Royo et al., 1996). Knowledge on LOXs has also been presented in grapevine (Podolyan et al., 2010), kiwifruit (Zhang et al., 2006), rice (Umate, 2011), apple (Vogt et al., 2013), soybean (Shin et al., 2008), cucumber (Liu et al., 2011), and olive (Padilla et al., 2009, 2012).

Pepper (Capsicum annuum) is an economically important crop worldwide. It is used e.g. as food, spice, and in pharmacology. There are many biotic and abiotic factors constraining pepper production (Shipp et al., 1998; Pakdeevaraporn et al., 2005; Kulkarni \& Phalke, 2009; Kurunc et al., 2011). Despite increasing commercial significance of pepper, the molecular mechanisms underlying different plant processes are still unknown. For instance, to develop resistance against pathogens and insects, identifying genes involved in different defence mechanisms in pepper is important.

To date, no comprehensive knowledge on the pepper LOX gene family is available. One 9-LOX, CaLOX1, involved in defence and cell-death responses against pathogens has been reported (Hwang \& Hwang, 2010). Recently, a second member of the LOX gene family (CaLOX2; Capana03g000103) was identified, playing a role in JA-regulated defence against Western flower thrips (Frankliniella occidentalis) (Sarde et al., 2018). Therefore, there is a need of a genome-wide survey of the LOX gene family of pepper. Here, we performed comparative genomics and domain-scan analyses for identification and classification of the LOX gene family in pepper. To investigate the conservation levels of pepper LOXs compared to known LOXs of other plant species, we subjected pepper LOXs to sequence analysis, phylogenetic analysis and homology modelling. Furthermore, to investigate the role of pepper LOXs in defence mechanisms, we examined their expression upon two treatments: exogenous JA application and exposure to feeding by a natural inducer of JA, the cell-content feeding insect Western flower thrips (WFT). WFT was selected because it is a major pest on 
pepper and well-known to induce JA signaling (Hickman et al., 2017; Steenbergen et al., 2018). The resulting data provide insights into putative functions of these genes in pepper.

\section{Materials and Methods}

\section{Sequence acquisition and identification of pepper LOXs}

Protein sequences of tomato (Solanum lycopersicum) lipoxygenases were obtained from the Ensembl Plants database (http://www.ensembl.org) (Yates et al., 2016). LOX sequences from Brassica oleracea, Brassica napus, Brassica rapa, Arabidopsis thaliana, Nicotiana attenuata, Nicotiana tabacum, Solanum tuberosum, Zea mays and Actindia deliciosa, were downloaded from NCBI (http://www.ncbi.nlm.nih.gov/). Oryza sativa and Cucumis melo LOX sequences were retrieved from the Rice Genome Annotation Project (http://rice.plantbiology.msu.edu/) and the Melonomics database (http://melonomics.net/), respectively. Two main approaches were used for the identification of the pepper LOX gene family. First, BLAST searches were performed locally on the Capsicum annuum L. Zunla-1 proteome (Qin et al., 2014) using Tomato LOX proteins as queries. Second, the Capsicum annuum L. Zunla-1 proteome was entirely analyzed for the presence of lipoxygenase gene family signature domains, LOX and PLAT/LH2 (polycystin-1, lipoxygenase, a-toxin domain or lipoxygenase homology) using the Pfam database (v27.0) in the CLC Bioworkbench (https://www.qiagenbioinformatics.com/).

\section{Sequence alignment of lipoxygenases}

Alignment of LOX protein sequences was performed using the MUSCLE tool (Edgar, 2004) with default settings. Editing and visualization of alignment was produced in GENEDOC (Nicholas et al., 1997). Sequence logos of conserved regions in pepper LOX proteins were generated by Weblogo 3.3 (Crooks et al., 2004).

\section{Phylogenetic analysis of plant LOXs}

Seventy-two plant LOX protein sequences were analyzed, including one known pepper LOX, CaLOX1(L) (L stands for 'literature') (Hwang \& Hwang, 2010) and eight pepper LOXs identified in the present study. A Maximum likelihood tree using WAG-model (Hall (2013), with 1000 bootstrap replicates was generated using MEGA 7.0 (Kumar et al., 2016). The tree was edited with the Figtree tool (http://tree.bio.ed.ac.uk/software/figtree/). 


\section{Sequence analysis and identification of conserved sequences}

Conserved sequences and pivotal amino acids were identified by manual observations on pepper LOX alignments in GENEDOC (Nicholas et al., 1997). Molecular weight and isoelectric point of pepper LOX proteins were calculated by protein isoelectric point calculator (Kozlowski, 2016). Subcellular localization analysis was performed using TargetP 1.1 (http://www.cbs.dtu.dk/services/TargetP/).

\section{Homology modeling of CaLOX1 and CaLOX2 protein}

We generated a protein structural model of CaLOX1 and CaLOX2 using the I-TASSER (Roy et al., 2010) database and the resulting model was visualized with YASARA (Krieger et al., 2002).

\section{Plant growth conditions, thrips rearing and bioassays}

Sweet pepper [Capsicum annuum (Mandy variety, Rijk Zwaan (De Lier, The Netherlands))] plants were grown in a greenhouse at $23-25^{\circ} \mathrm{C}, 70 \pm 10 \%$ relative humidity and 16L:8D photoperiod. Four-week-old plants were used in the experiments for both treatments. Western flower thrips (WFT; Frankliniella occidentalis) were reared on bean pods (Phaseolus vulgaris) in a climate-controlled cabinet $\left(25 \pm 2{ }^{\circ} \mathrm{C}, 70 \pm 10 \%\right.$ relative humidity, L16:8D photoperiod). For thrips treatment in the gene expression experiment, five $2^{\text {nd }}$ instar thrips larvae were placed in clip cages and used for infestation on one of the first two true leaves. Empty clip cages were used on control plants for each time point. Samples were harvested at 0, 2, 4, 6, 8, 10 and 24 hours post infestation, frozen in liquid nitrogen and stored at $-80^{\circ} \mathrm{C}$.

\section{RNA extraction and qRT-PCR}

Transcriptional responses of pepper LOXs in response to JA treatment $(100 \mu \mathrm{M})$ and thrips feeding were assessed by qRT-PCR. For JA-treatment, plants were dipped in $100 \mu \mathrm{M}$ of JA (treatment) or mock-treated with water (control), both mixed with $0.1 \%$ of Tween20. One of the first two true leaf samples were harvested at 0, 0.5, 1, 2, $3,6,8,10$ and 24 hours post JA application, frozen in liquid nitrogen and stored at $-80^{\circ} \mathrm{C}$. For both treatments (JA and thrips), control samples were harvested at each time point to rule out the effect of circadian rhythm on the expression of LOX genes. Four to five biological replicates (individual plants) were harvested and analysed for each time point and treatment. Each biological replicate comprises one individual plant. Bioline kit (ISOLATE II RNA Plant Kit), in accordance to its protocol, was used for RNA extraction. cDNA synthesis was executed with $1 \mathrm{\mu g}$ of total RNA with BioRad iScript cDNA synthesis kit. For qPCR, a reaction mixture comprising of $12.5 \mu \mathrm{l}$ of SYBR Green (Bioline), $1 \mu \mathrm{l}(10 \mu \mathrm{M})$ of forward and reverse primers, $5.5 \mu \mathrm{l}$ RNase free-water and $5 \mu \mathrm{l}$ cDNA was used. The data normalization was performed with a reference gene, CaActin. The PCR cycle conditions used were $95^{\circ} \mathrm{C}$ for 3 mins, fol- 
lowed by 40 cycles of $95^{\circ} \mathrm{C}$ for $15 \mathrm{~s}$, and $60^{\circ} \mathrm{C}$ for $45 \mathrm{~s}$. Melt curves for each gene were recorded at the end of each cycle. All primers used for qPCR are presented in Supplementary file S1.

Relative gene expression was studied using the geometric mean of $\mathrm{Ct}$ (threshold cycles) values (Vandesompele et al., 2002) from the reference gene CaActin using the 2- ${ }^{\Delta \Delta C t}$ method (Livak \& Schmittgen, 2001).

\section{Statistical analysis}

The gene expression data were subjected to a Student's $t$-test.

\section{Results and Discussion}

\section{Identification of lipoxygenase gene family in pepper}

A genome-wide search for lipoxygenase genes in pepper was performed by implementing two main approaches: homology search and scanning of the pepper proteome for the presence of "lipoxygenase" and "PLAT/LH2" domains. Both approaches resulted in the identification of eight lipoxygenases in the Capsicum annuum $L$. Zunla-1 proteome (Table 1). Several proteins depicting the presence of either one lipoxygenase domain or the PLAT/LH2 domain were excluded from analysis based on arguments of Chen et al. (2015).

The total number of LOXs in pepper (8) is similar to that in tomato (7). This number is also close to the number in Arabidopsis (6) (Umate, 2011) and kiwifruit (6) (Zhang et al., 2006), double the number in olive (4) (Padilla et al., 2009, 2012) and much lower than in melon (18) (Zhang et al., 2014), cucumber (23) (Liu et al., 2011) and grapevine (18) (Podolyan et al., 2010). This diverse number of LOXs in different plant species indicates that this gene family has not been conserved during evolution, despite similarities in biochemical functions of the gene family in different plant species (Feussner \& Wasternack, 2002).

Genomic and proteomic features of the pepper LOX gene family do not differ much (Table 1). At the genomic level, the number of introns varies between 7 and 9, whereas, ORF (Open Reading Frame) length ranges from a minimum of 2379 bp to a maximum of $2748 \mathrm{bp}$. Most of the pepper LOXs are located on Chromosomes 1 and 3, with the exception of CaLOX8 (Capana11g000928) on Chromosome 11. At the protein level, LOX length varied between 792 and 915 aa, the predicted isoelectric point $(\mathrm{PI})$ ranged between 5.4 and 7.5 and the predicted molecular weight of the proteins ranged from 89959 to $104131 \mathrm{Da}$. Sequence comparison among pepper LOXs at the protein level shows high sequence identity $(33-70 \%)$ and similarity (48 - 77 $\%$ ) (Table 2). Taken together, these genomic and proteomic features show a close relation among the pepper $L O X s$, indicative of a gene family. 


\section{Phylogenetic analysis of lipoxygenases}

To determine the evolutionary relationship and predict the classification of pepper LOXs, a maximum-likelihood phylogenetic tree with 1000 bootstraps was generated. For this, we used sixty-four previously known plus eight pepper LOX protein sequences from twelve different plant species, comprising monocots and dicots. The tree explicitly categorizes plant LOXs into 9-LOXs, 13-LOXs and uncharacterized LOXs. From the identified eight pepper LOXs, four LOXs (CaLOX1, CaLOX3, CaLOX4 and CaLOX5) are characterized, including the previously described CaLOX1(L) (Hwang \& Hwang, 2010) into the 9-LOX group and four other LOXs (CaLOX2, CaLOX6, CaLOX7 and CaLOX8) into the 13-LOX group (Fig. 1). Moreover, upon closer examination of the 9- and 13-LOXs major clades, explicit sub-clades of monocot and dicot LOXs are formed indicating that this gene family has evolved differently in monocots and dicots (Fig. 1).

In the 13-LOX clade, pepper LOXs group with well-characterized Solanaceae 13LOXs like SILOXD, StLOXH3, NaLOX3, SILOXF, NaLOX2, StLOXH1 and SILOXC (Fig. 1). These clusters or sub-clusters among known LOXs and newly identified LOXs may be useful to predict biochemical features and molecular functions of the newly identified pepper LOXs. CaLOX2 clusters with SILOXD, StLOXH3 and NaLOX3, wellknown to be involved in JA biosynthesis (Royo et al., 1996; Halitschke \& Baldwin, 2003; Kessler, 2004; Yan et al., 2013) suggesting that CaLOX2 has a similar function. This matches with our recent study experimentally confirming that CaLOX2 is involved in JA biosynthesis upon thrips feeding (Sarde et al., 2018). Virus-induced gene silencing of CaLOX2 led to disruption of the jasmonate pathway resulting in enhanced performance of thrips. CaLOX6 clusters with SILOXF, known to be involved in systemic resistance to Pseudomonas putida BTP1 (Mariutto et al., 2011). CaLOX7 groups with NaLOX2, StLOXH1 and SILOXC. These three LOXs are involved in the biosynthesis of green leaf volatiles (Leon et al., 2002; Chen et al., 2004; Allmann et al., 2010; VanDoorn et al., 2010). CaLOX8 seems to be related to AtLOX6, known to provide resistance against biotic and abiotic stresses through oxylipin biosynthesis in roots (Grebner et al., 2013). 


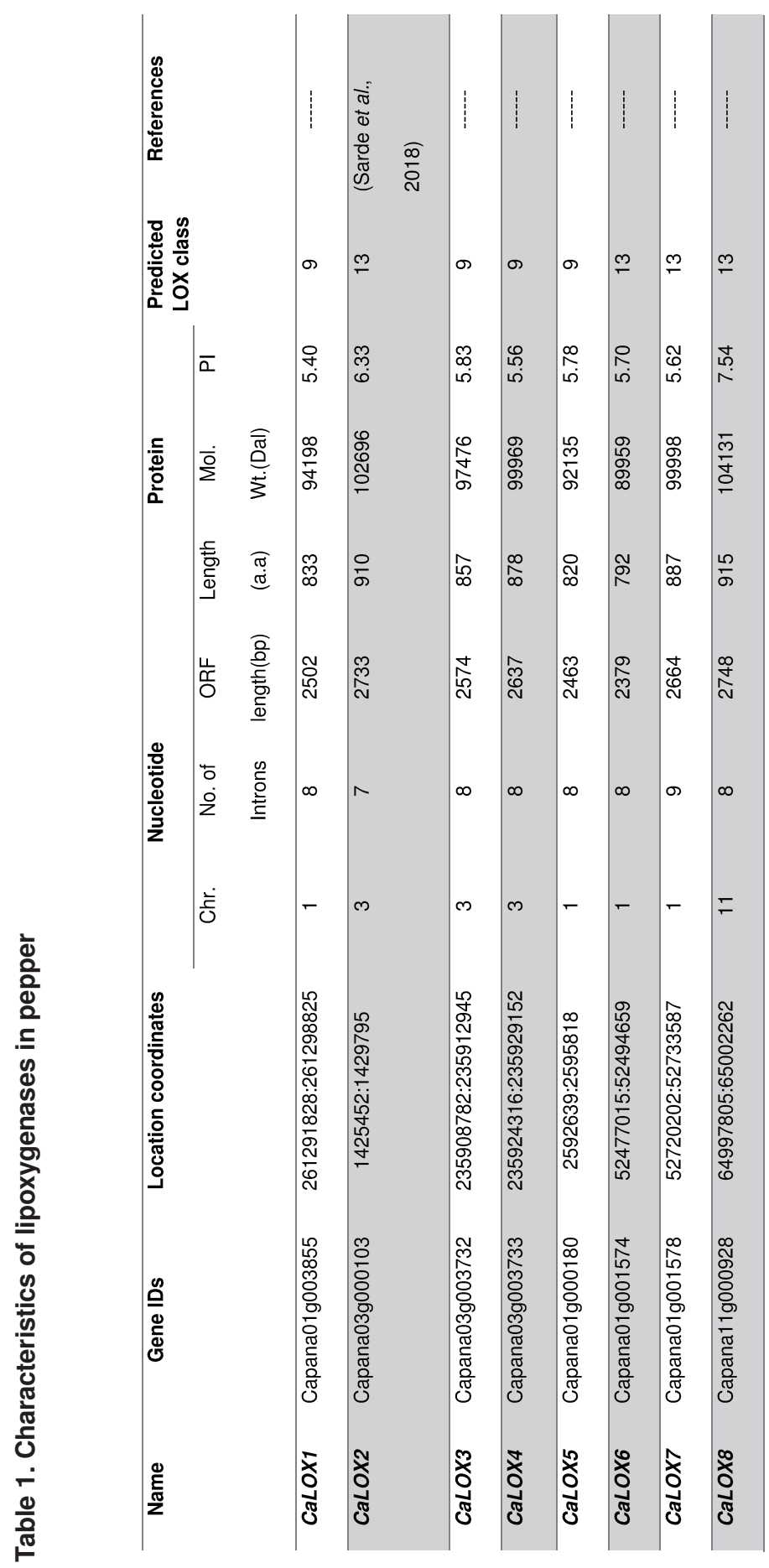


Table 2. Pepper LOX protein identities and similarities (\%). High, intermediate and low similarity/identity of genes is shown in green, yellow and red color, respectively.

\begin{tabular}{|c|c|c|c|c|c|c|c|c|}
\hline $\begin{array}{c}\text { Gene } \\
\text { ids }\end{array}$ & CaLOX5 & CaLOX1 & CaLOX3 & CaLOX4 & CaLOX7 & CaLOX6 & CaLOX8 & CaLOX2 \\
\hline CaLOX5 & & 57 & 52 & 53 & 36 & 33 & 38 & 38 \\
\hline CaLOX1 & 72 & & 59 & 64 & 39 & 35 & 38 & 40 \\
\hline CaLOX3 & 70 & 73 & & 70 & 39 & 36 & 37 & 38 \\
\hline CaLOX4 & 69 & 77 & 83 & & 38 & 35 & 38 & 39 \\
\hline CaLOX7 & 51 & 56 & 55 & 56 & & 70 & 45 & 45 \\
\hline CaLOX6 & 48 & 50 & 50 & 48 & 77 & & 42 & 40 \\
\hline CaLOX8 & 53 & 54 & 54 & 55 & 65 & 59 & & 52 \\
\hline \multirow[t]{2}{*}{ CaLOX2 } & 54 & 57 & 57 & 56 & 62 & 56 & 69 & \\
\hline & \multicolumn{8}{|c|}{ Sequence similarity } \\
\hline
\end{tabular}

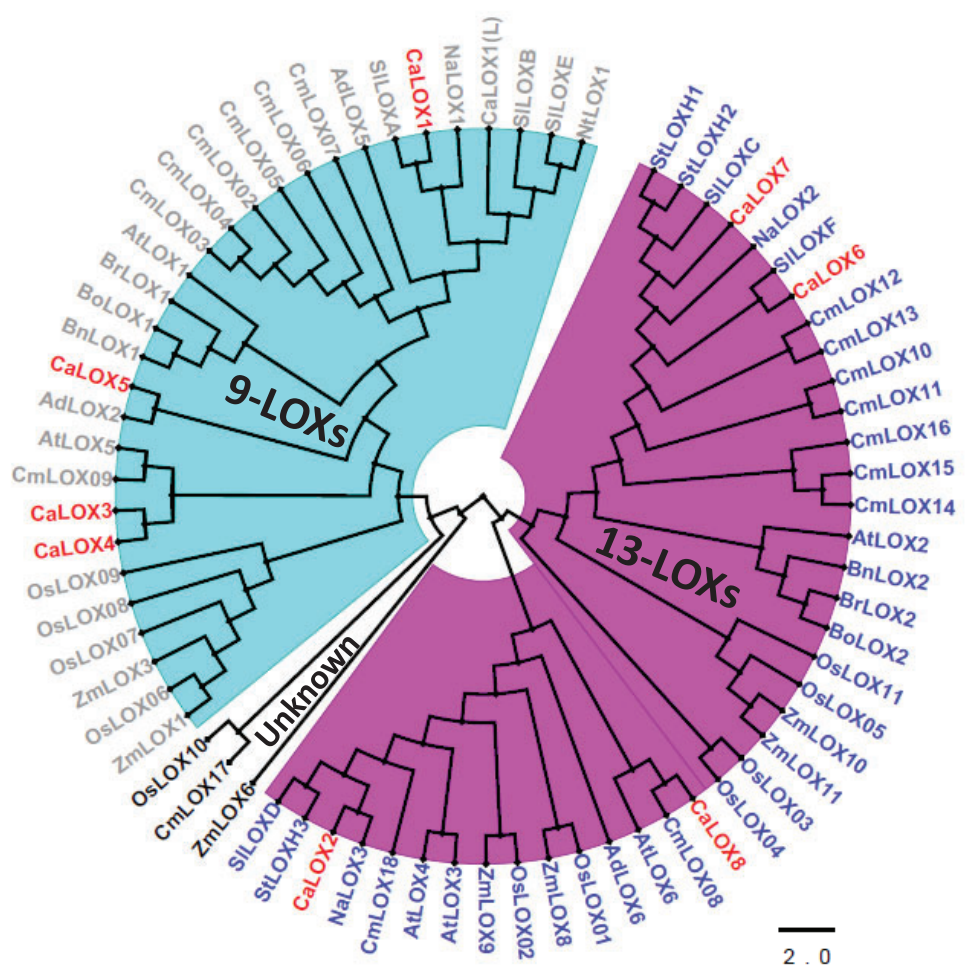

Figure 1: Phylogenetic analysis of plant lipoxygenases. The evolutionary relationship between pepper and other LOX proteins. The tree was generated by MEGA 7 using Maximum Likelihood method with 1000 bootstraps and viewed in Figtree. The scale bar represents the branch length. Different classes of LOXs are depicted in different colors, 13-LOXs in purple; 9-LOXs in blue; unclassified without color. Identified pepper LOXs are highlighted in red color. Species abbreviations used for phylogeny are as follows. At: Arabidopsis thaliana, Bo: Brassica oleracae, Bn: Brassica napus, Br: Brassica rapa, SI: Solanum lycopersicum, St: Solanum tuberosum, Na: Nicotiana attenuata, Nt: Nicotiana tabacum, Ca: Capsicum annuum, Os: Oryza sativa, Zm: Zea Mays, Cm: Cucumis melo, Ad: Actindia deliciosa. 
Also in the 9-LOX clade, pepper LOXs cluster with functionally characterized LOXs of other plant species such as AtLOX5, AdLOX2, SILOXA, SILOXB (Fig. 1). AdLOX2, that mediates the generation of C6 aldehydes in kiwifruit (Zhang et al., 2009), clusters with CaLOX5, suggesting that CaLOX5 has a similar function. CaLOX3 and CaLOX4 form a major clade with AtLOX5 and CmLOX09. AtLOX5 is involved in lateral root development and defence responses (Vellosillo et al., 2007). Additionally, relatedness of CaLOX3 and CaLOX4 to each other, suggests that they may be isoforms mediating the same biological process. Furthermore, clustering together of identified CaLOX1 from Capsicum annuum Zunla-1 proteome (Qin et al., 2014) and known CaLOX$1(\mathrm{~L})$ reflects their similarity/relatedness, suggesting them to be the same protein. Hwang and Hwang (2010) identified CaLOX1(L) independently from cDNA clones and reported it to be involved in defence and cell-death responses against pathogens. Furthermore, CaLOX1 identified here and the previously reported CaLOX1(L) (Hwang \& Hwang, 2010) cluster with LOXs like SILOXA and SILOXB, two LOXs that are up-regulated in ripening tomato fruits (Ferrie et al., 1994; Griffiths et al., 1999). Nevertheless, taken together, the predicted functions of pepper LOXs require further experimental validation to characterize their molecular functions, as reported for CaLOX1(L) and CaLOX2 (Hwang \& Hwang, 2010; Sarde et al., 2018).

Finally, the reported uncharacterized LOXs like OsLOX10, CmLOX17 and ZmLOX6 clearly form an outgroup from the 9- and 13-LOXs (Liu et al., 2011; Zhang et al., 2014; Cao et al., 2016).

\section{Sequence analysis consolidates phylogenetic classification of pepper LOXs}

The lipoxygenase family of pepper (CaLOX1 - CaLOX8) is highly conserved with variable sequence identities and similarities with each other (Table 2). It is known that, based on degree of sequence similarity and presence/absence of chloroplast-transit peptide, LOXs are classified into Type-1 or Type-2 (Feussner \& Wasternack, 2002; Porta \& Rocha-Sosa, 2002). Type-1 LOXs show high sequence similarity (>75\%) in the absence of a chloroplast-transit peptide; in contrast, Type-2 LOXs show low sequence similarity and the presence of a chloroplast-transit peptide. The pepper LOXs CaLOX1 and CaLOX3, CaLOX4 and CaLOX5, exhibit high sequence similarity (>70\% ) and identity (>52\%) with each other compared to other LOXs. In contrast, CaLOX2 and CaLOX6, CaLOX7 and CaLOX8 show low sequence similarity among themselves with the exception of CaLOX6 and CaLOX7. CaLOX6 and CaLOX7 show high sequence similarity and identity among themselves, but not when compared to the rest of the LOXs, suggesting that CaLOX6 and CaLOX7 are isoforms of each other. Furthermore, the presence of a chloroplast-transit peptide in sequences of CaLOX2 and CaLOX6, CaLOX7 and CaLOX8 suggests that they are localized in the chloroplast. Collectively, sequence similarity and sub-cellular localization analysis indicates classification of CaLOX1, CaLOX3, CaLOX4 and CaLOX5 into Type-1 and CaLOX2, 
CaLOX6, CaLOX7 and CaLOX8 into Type-2.

Furthermore, plant LOXs are also classified into 9- and 13-LOXs, based on their positional specificity of action on the substrate (Feussner \& Wasternack, 2002). The presence of Phe608/His608 or Val608 residue predicts LOX activity as 13- or 9-LOX, respectively. Multiple sequence alignment of all pepper LOXs clearly shows the occurrence of valine in CaLOX1 and CaLOX3, CaLOX4 and CaLOX5 classifying them as 9-LOXs and phenylalanine in CaLOX2 and CaLOX6, CaLOX7 and CaLOX8 classifying them as 13-LOXs (Fig. S1). This agrees with the observation that all Type-2 LOXs identified so far are 13-LOXs (Feussner \& Wasternack, 2002).

Therefore, both classification methods provide consensus on distribution of pepper LOXs into different classes, thus consolidating our methodology and predictions. Moreover, it also suggests to use the parameters from both approaches in the future for classification of plant lipoxygenases.

\section{High conservation of motifs and pivotal amino acids}

Lipoxygenases are characterized by the presence of a 38-residue representative sequence, a substrate-binding domain, an oxygen binding domain and a C-terminal motif (Padilla et al., 2009, 2012). The highly representative 38-residue sequence in lipoxygenases is important for stability of lipoxygenases. In addition, the enzymatic activity or efficiency of lipoxygenases can be severely affected if any of the residues of this sequence is substituted (Chen et al., 2015). This sequence is highly conserved in all the predicted pepper LOXs (Fig. 2A, 2E \& 2F). Also, the other motifs like substrate binding, oxygen binding and the C-terminal are conserved among all pepper LOXs (Fig. 2A-F).

Among the conserved amino acids, the three histidine residues (including two from the representative 38-residue sequence) His499, His504, His690 with Asn694 and lle839 are identified to be vital for binding to non-heme iron (Steczko et al., 1992; Boyington et al., 1997; Feussner \& Wasternack, 2002; Porta \& Rocha-Sosa, 2002; Padilla et al., 2012).

All these five amino acids appear to be conserved in the pepper LOXs (Fig. S1) with an exception for lle839 in CaLOX7. Substitution of C-terminal isoleucine with any other amino acid except valine led to inactivation of lipoxygenases, whereas, substitution with valine had positive consequences for enzymatic activity (Chen et al., 1994). Therefore, the absence of a C-terminal motif and the presence of lle839, essential for non-heme iron binding, leads us to suggest that CaLOX7 may have an altered enzymatic activity. 
A. Motif-I

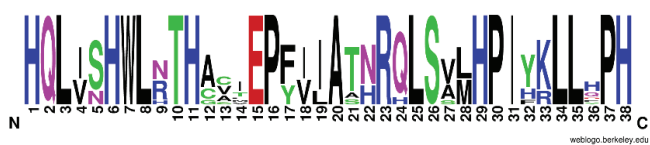

Representative 38-residue

B. Motif-II

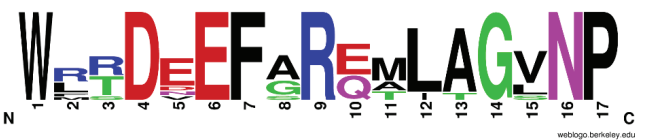

Substrate-binding

C. Motif-III

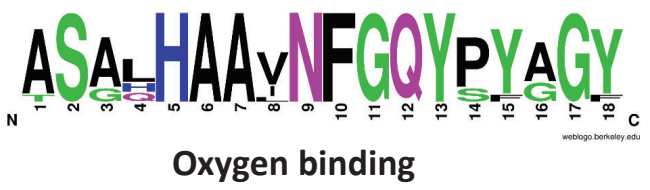

D. Motif-IV

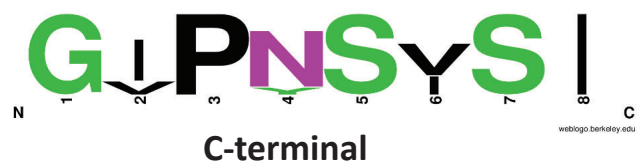

\section{E. CaLOX1}

\section{F. CaLOX2}

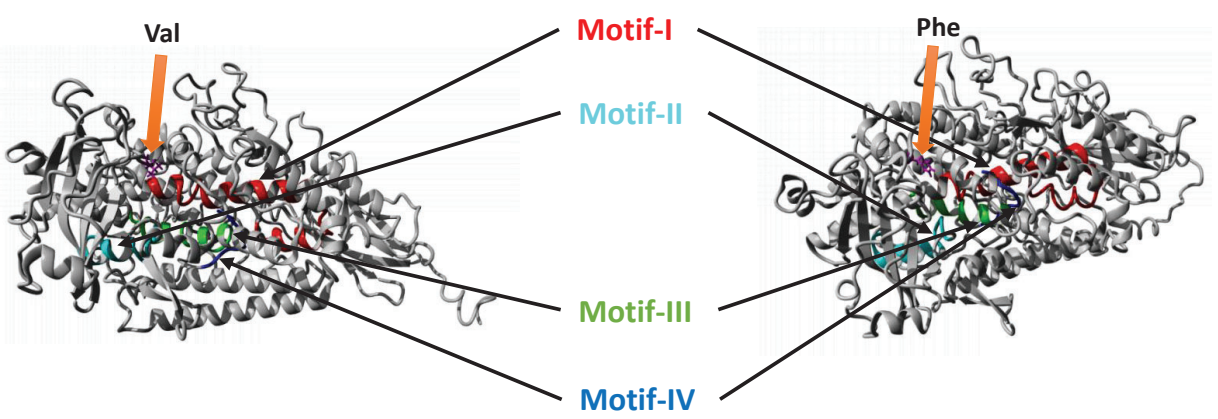

Figure 2. Conservation of sequence motifs in pepper lipoxygenases (A-D) and protein models of CaLOX1 and CaLOX2 (E-F). Highly representative 38-residue motif (A), substrate binding (B), oxygen binding (C) and C-terminal (D) motifs are highly conserved. Protein model of a 9-LOX CaLOX1 (E) and 13-LOX CaLOX2 (F) depicting conservation of highly representative 38-residue (red), substrate binding (cyan), oxygen binding (green) and C-terminal motif (blue) motifs. 13- or 9-LOX activity determinant Phe or Val residue, respectively are depicted. 
Moreover, the conserved Val608 or Phe608/His608 residue that are indicative for 9- or 13-LOX activity, respectively (Sloane et al., 1991; Hornung et al., 1999; Padilla et al., 2009, 2012), are found highly conserved in pepper LOXs (Fig. 2E-F and Fig. S1). The determinant residues for inverse substrate orientation and S-stereospecificity of LOXs, Arg and Ala, respectively (Hornung et al., 1999; Coffa \& Brash, 2004) are well-conserved as well in pepper LOXs (Supplemental Fig. S1). Taken together, the conservation of motifs and pivotal amino acids suggests that functions of pepper LOXs are conserved to their respective homologs in other plant species.

\section{Expression pattern of lipoxygenases upon JA application and thrips feeding}

qRT-PCR was performed to investigate the expression dynamics of pepper LOXs over time upon thrips feeding or exogenous JA application. Upon thrips feeding, two 13-LOXs (CaLOX2 and CaLOX7) are up-regulated for most of the analyzed time points (Fig. 3). Induction of CaLOX2 occurred after $2 \mathrm{~h}$ of thrips feeding and remained up-regulated. This gene's involvement in JA biosynthesis has been experimentally supported (Sarde et al., 2018). CaLOX7 is significantly up-regulated at $4 \mathrm{~h}$ after the start of feeding and remained up-regulated throughout the period suggesting that it may have a role in defence. CaLOX6 is significantly up-regulated after $10 \mathrm{~h}$ of feeding. In contrast, all other LOXs, i.e. CaLOX1,CaLOX3, CaLOX4 and CaLOX8 did not show induction over time (Fig. 3). CaLOX4 and CaLOX8 are significantly down-regulated after $8 \mathrm{~h}$ of thrips feeding. CaLOX5 expression is not shown due to its unstable expression resulting in a high degree of variation. This instability of CaLOX5 expression was also confirmed by its expression pattern in an RNA-seq dataset (Sarde et al., unpublished data). In Arabidopsis, it is well-known that LOX expression is triggered following application of JA due to presence of a positive feedback loop that amplifies JA responses (Hickman et al., 2017). Upon exogenous JA application, CaLOX2, known to be involved in JA biosynthesis (Sarde et al., 2018), shows significant induction after $2 \mathrm{~h}$ which was maintained until $6 \mathrm{~h}$ after JA application with exception at $3 \mathrm{~h}$ (Fig. 4). This instant up- and downregulation of CaLOX2, suggests involvement of some feedback mechanism in JA-biosynthetic pathway. CaLOX6 and CaLOX7 are also upregulated upon JA application. Both of them exhibit high expression levels at similar time points i.e., $8 \mathrm{~h}$ and $24 \mathrm{~h}$ after $\mathrm{JA}$ application. In contrast, the other $L O X$ genes, i.e. CaLOX1, CaLOX3, CaLOX4 and CaLOX8, were not up-regulated at any time point, but exhibited down-regulation at several time points (Fig. 4). 

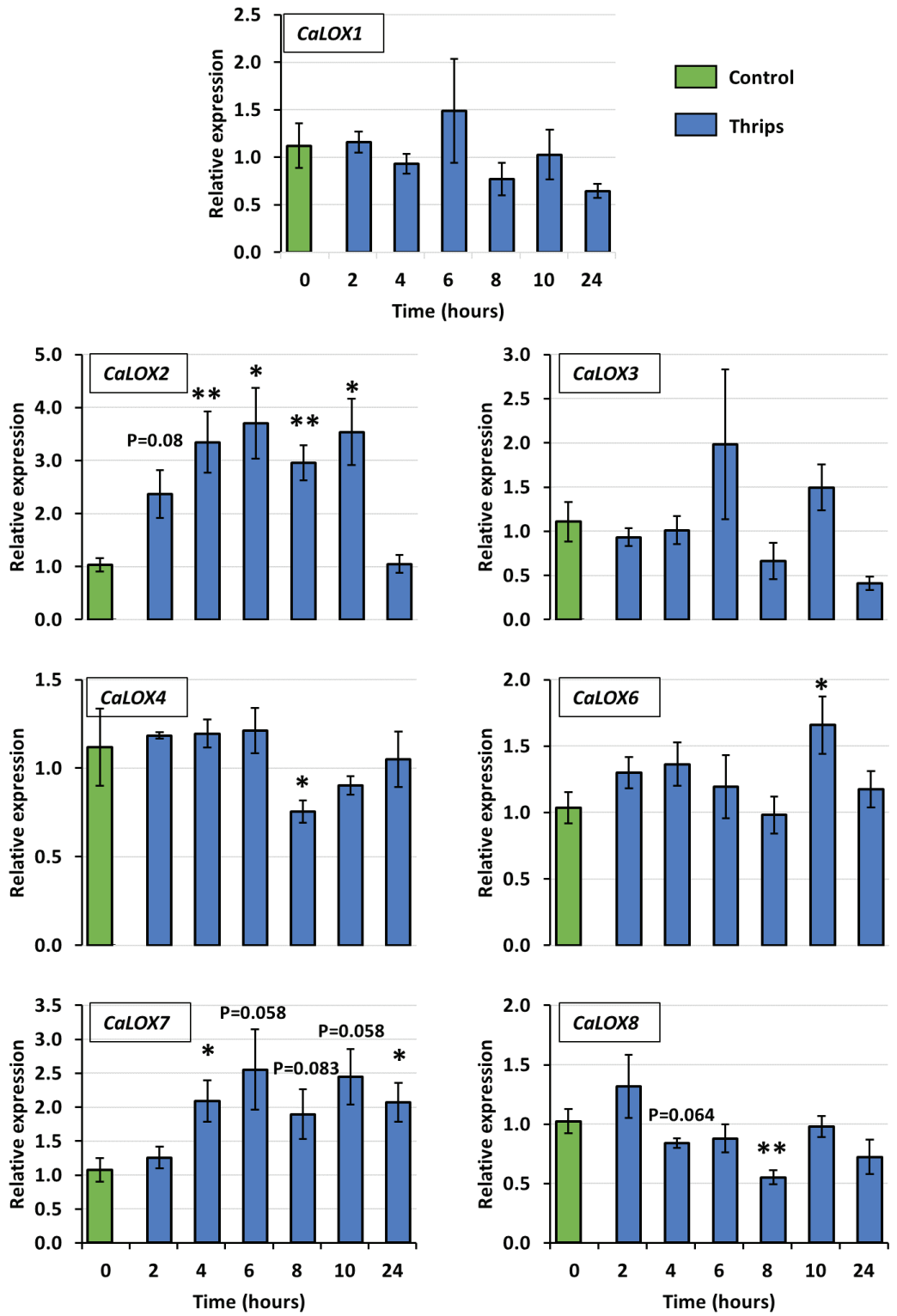

Figure 3. Quantitative RT-PCR (RT-qPCR) of pepper lipoxygenase genes in sweet pepper leaves in response to thrips ( $F$. occidentalis) feeding. Five $2^{\text {nd }}$ instar thrips larvae in a clip cage fed on the first true leaf of four-week-old pepper plants. Clip cages without thrips were used on control plants. Expression of the housekeeping gene CaActin was used to normalize the expression level of each LOX gene at each time point. Relative expression compared to the control for the same time point is presented. Bars represent means \pm SE of 4-5 biological replicates. Bars marked with asterisks indicate significant differences (Student's $t$-test) to corresponding control samples for the same time point, ${ }^{*} \mathrm{P}<0.05$, ${ }^{* *} \mathrm{P}<0.01$. For bars without asterisk or $\mathrm{P}$ value, the $\mathrm{P}$ value is $>0.10$. 

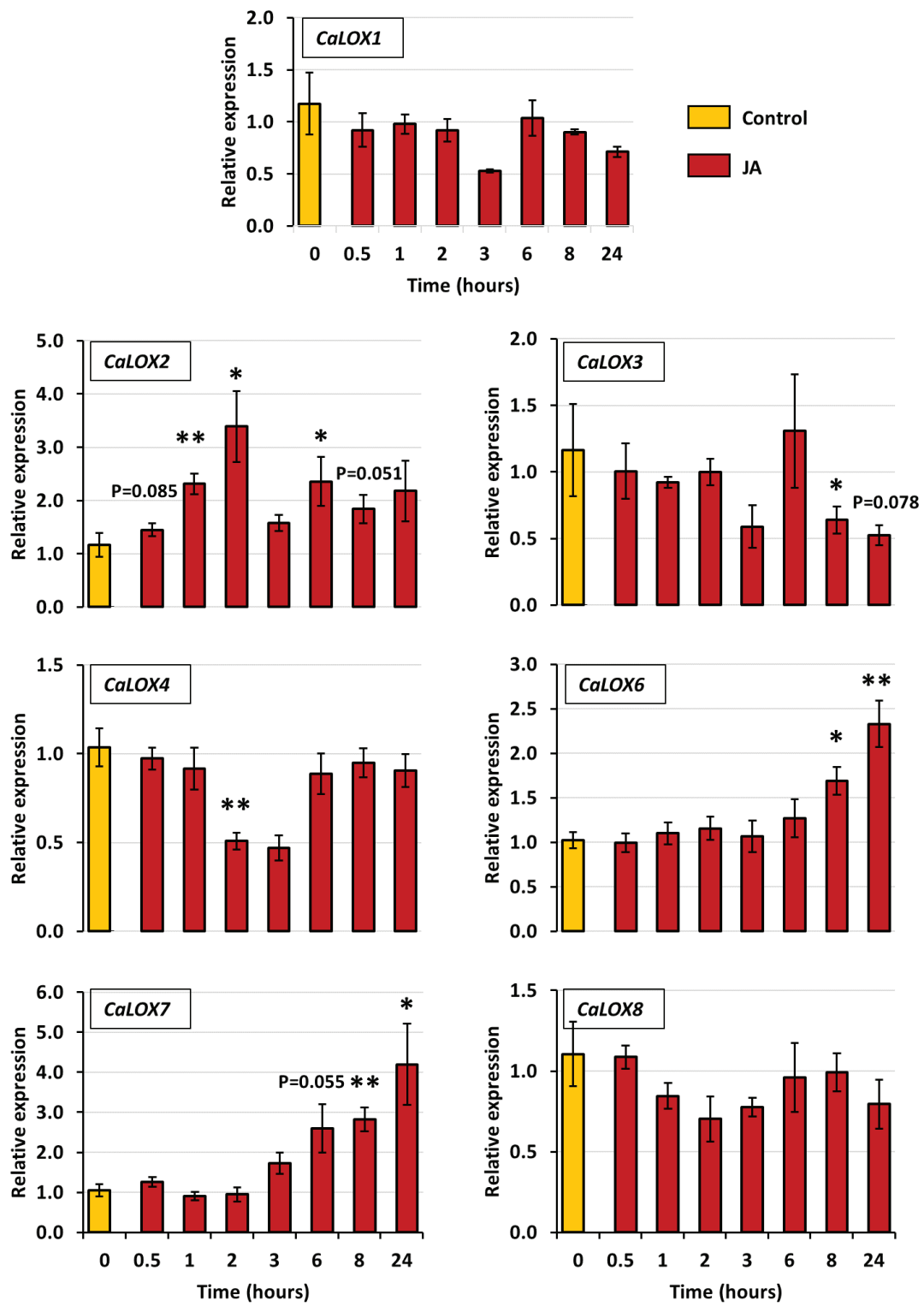

Figure 4. Quantitative RT-PCR (RT-qPCR) of pepper lipoxygenase genes in sweet pepper leaves in response to exogenous JA application. Pepper plants were dipped in water + Tween20 (control) or $100 \mu \mathrm{M} \mathrm{JA}+$ Tween20 (treatment). Expression of the housekeeping gene CaActin was used to normalize the expression level of each LOX gene at each time point. Relative expression compared to the control for the same time point is presented. Bars represent means \pm SE of 4-5 biological replicates. Bars marked with asterisks indicate significant differences (Student's $t$-test) to corresponding control samples for the same time point, * $P<0.05,{ }^{* *} P<0.01$. For bars without asterisk or $P$ value, the $P$ value is $>0.10$. 
In general, the 9-LOXs in pepper (CaLOX1, CaLOX3 and CaLOX4) did not show any induction, but rather down-regulation at certain time points in both treatments, i.e. JA application and thrips feeding. This fits to the fact that 9-LOXs are especially involved in functions such as plant-pathogen interactions, storage of proteins and tuber development (Feussner \& Wasternack, 2002). In contrast, the 13-LOXs were more responsive to both treatments, except CaLOX8. Similarity of CaLOX7 to NaLOX2 and SILOXC (Fig. 1), both known to be involved in the biosynthesis of green leaf volatiles (GLVs) (Chen et al., 2004; Allmann et al., 2010; VanDoorn et al., 2010; Shen et al., 2014), and it's up-regulation upon both thrips feeding and JA application (Fig. 3 \& 4) suggest a role of CaLOX7 in the biosynthesis of GLVs in pepper. Additionally, in tomato SILOXC-antisense lines, low expression of both SILOXC and SILOXF resulted in decreased levels of C5 and C6 leaf volatiles, suggesting a possible synergistic involvement of SILOXC and SILOXF in the biosynthesis of these plant volatiles (Shen et al., 2014). Therefore, the similarity of CaLOX6 to SILOXF (Fig. 1) and its induction upon both JA application and thrips feeding makes it a potential candidate to test for its synergistic role with CaLOX7 in volatile biosynthesis (Fig. 3 and 4).

\section{Conclusion}

In conclusion, this study has identified and classified eight LOXs in pepper. Phylogenetic analysis classified four LOXs as 9-LOXs (CaLOX1, CaLOX3, CaLOX4 and CaLOX5) and four others as 13-LOXs (CaLOX2, CaLOX6, CaLOX7 and CaLOX8) with predictions of their putative functions. Pepper LOX proteins are highly conserved in all lipoxygenase characteristics. Characterization of CaLOX2 encoding for a LOX that is involved in JA biosynthesis is confirmed by a recent experimental study through a combination of in-silico, transcriptional, behavioural, and chemical analyses plus silencing of CaLOX2 through Virus-Induced Gene Silencing (Sarde et al., 2018). For the other LOXs their function remains to be elucidated. High expression levels of 13LOXs in pepper with support of in-silico analysis predict potential candidate genes (CaLOX6 and CaLOX7) that code for enzymes involved in GLV biosynthesis in pepper. Finally, this comprehensive study provides a pepper LOX genes repository to further elucidate their functional roles in respective biological processes.

\section{Acknowledgements}

We thank Gerrie Wiegers for helping with the culture of thrips and Wageningen University \& Research experimental farm (Unifarm) for rearing the plants. This work is part of the Perspective programme Green Defences Against Pests that is (partly) financed by the Netherlands Organisation for Scientific Research (NWO, grant no. 
13555) and partly by the breeding companies Keygene, Syngenta and EastWest Seeds through NWO.

\section{References}

Allmann S, Halitschke R, Schuurink RC, Baldwin IT. 2010. Oxylipin channelling in Nicotiana attenuata: lipoxygenase 2 supplies substrates for green leaf volatile production. Plant Cell and Environment 33(12): 2028-2040.

Bailly C, Bogatek-Leszczynska R, Come D, Corbineau F. 2002. Changes in activities of antioxidant enzymes and lipoxygenase during growth of sunflower seedlings from seeds of different vigour. Seed Science Research 12(1): 47-55.

Barry CS, Giovannoni JJ. 2007. Ethylene and fruit ripening. Journal of Plant Growth Regulation 26(2): 143-159.

Bell E, Creelman RA, Mullet JE. 1995. A chloroplast lipoxygenase is required for wound-induced jasmonic acid accumulation in Arabidopsis. Proceedings of the National Academy of Sciences of the United States of America 92(19): 8675-8679.

Boyington JC, Gaffney BJ, Amzel LM. 1997. The three-dimensional structure of soybean lipoxygenase-1: An arachidonic acid 15-lipoxygenase. Eicosanoids and Other Bioactive Lipids in Cancer, Inflammation, and Radiation Injury 2, Pts a and B 400: 133-138.

Brash AR. 1999. Lipoxygenases: Occurrence, functions, catalysis, and acquisition of substrate. Journal of Biological Chemistry 274(34): 23679-23682.

Caldelari D, Wang GG, Farmer EE, Dong XN. 2011. Arabidopsis lox3 lox4 double mutants are male sterile and defective in global proliferative arrest. Plant Molecular Biology 75(12): 25-33.

Cao SX, Chen H, Zhang C, Tang YF, Liu JY, Qi HY. 2016. Heterologous expression and biochemical characterization of two lipoxygenases in Oriental Melon, Cucumis melo var. makuwa Makino. PLoS One 11(4): e0153801.

Chen GP, Hackett R, Walker D, Taylor A, Lin ZF, Grierson D. 2004. Identification of a specific isoform of tomato lipoxygenase (TomloxC) involved in the generation of fatty acid-derived flavor compounds. Plant Physiology 136(1): 2641-2651.

Chen XS, Kurre U, Jenkins NA, Copeland NG, Funk CD. 1994. cDNA cloning, expression, mutagenesis of C-terminal isoleucine, genomic structure, and chromosomal localizations of murine 12-lipoxygenases. Journal of Biological Chemistry 269(19): 13979-13987.

Chen Z, Chen X, Yan HW, Li WW, Li Y, Cai RH, Xiang Y. 2015. The Lipoxygenase gene family in Poplar: Identification, classification, and expression in response to MeJA treatment. PLoS One 10(4): e0125526.

Christensen SA, Huffaker A, Hunter CT, Alborn HT, Schmelz EA. 2016. A maize death acid, 10-oxo-11-phytoenoic acid, is the predominant cyclopentenone signal present during multiple stress and developmental conditions. Plant Signaling \& Behavior 11(2): e1120395.

Christensen SA, Huffaker A, Kaplan F, Sims J, Ziemann S, Doehlemann G, Ji L, Schmitz RJ, Kolomiets MV, Alborn HT, Mori N, Jander G, Ni X, Sartor RC, Byers S, Abdo Z, Schmelz EA. 2015. Maize death acids, 9-lipoxygenase-derived cyclopente(a)nones, display activity as cytotoxic phytoalexins and transcriptional mediators. Proceedings of the National Academy of Sciences of the United States of America 112(36): 11407-11412.

Coffa G, Brash AR. 2004. A single active site residue directs oxygenation stereospecificity in 
lipoxygenases: Stereocontrol is linked to the position of oxygenation. Proceedings of the National Academy of Sciences of the United States of America 101(44): 15579-15584.

Crooks GE, Hon G, Chandonia JM, Brenner SE. 2004. WebLogo: A sequence logo generator. Genome Research 14(6): 1188-1190.

Edgar RC. 2004. MUSCLE: a multiple sequence alignment method with reduced time and space complexity. BMC Bioinformatics 5: 113.

Ferrie BJ, Beaudoin N, Burkhart W, Bowsher CG, Rothstein SJ. 1994. The cloning of two tomato lipoxygenase genes and their differential expression during fruit ripening. Plant Physiology 106(1): 109-118.

Feussner I, Wasternack C. 2002. The lipoxygenase pathway. Annual Review of Plant Biology 53: $275-297$.

Grebner W, Stingl NE, Oenel A, Mueller MJ, Berger S. 2013. Lipoxygenase6-dependent oxylipin synthesis in roots is required for abiotic and biotic stress resistance of Arabidopsis. Plant Physiology 161(4): 2159-2170.

Griffiths A, Barry C, Alpuche-Solis AG, Grierson D. 1999. Ethylene and developmental signals regulate expression of lipoxygenase genes during tomato fruit ripening. Journal of Experimental Botany 50(335): 793-798.

Halitschke R, Baldwin IT. 2003. Antisense LOX expression increases herbivore performance by decreasing defense responses and inhibiting growth-related transcriptional reorganization in Nicotiana attenuata. Plant Journal 36(6): 794-807.

Hall BG. 2013. Building phylogenetic trees from molecular data with MEGA. Molecular Biology and Evolution 30(5): 1229-1235.

Hickman R, Van Verk MC, Van Dijken AJH, Mendes MP, Vroegop-Vos IA, Caarls L, Steenbergen M, Van der Nagel I, Wesselink GJ, Jironkin A, Talbot A, Rhodes J, De Vries M, Schuurink RC, Denby K, Pieterse CMJ, Wees SCM. 2017. Architecture and dynamics of the jasmonic acid gene regulatory network. Plant Cell 29(9): 2086-2105.

Hornung E, Walther M, Kuhn H, Feussner I. 1999. Conversion of cucumber linoleate 13-lipoxygenase to a 9-lipoxygenating species by site-directed mutagenesis. Proceedings of the National Academy of Sciences of the United States of America 96(7): 4192-4197.

Hwang IS, Hwang BK. 2010. The Pepper 9-Lipoxygenase gene CaLOX1 functions in defense and cell death responses to microbial pathogens. Plant Physiology 152(2): 948-967.

Kessler A. 2004. Silencing the jasmonate cascade: Induced plant defenses and insect populations (vol 305, pg 665, 2004). Science 306(5704): 2042-2042.

Kolomiets MV, Hannapel DJ, Chen H, Tymeson M, Gladon RJ. 2001. Lipoxygenase is involved in the control of potato tuber development. Plant Cell 13(3): 613-626.

Kozlowski LP. 2016. IPC - Isoelectric Point Calculator. Biology Direct 11: 55.

Krieger E, Koraimann G, Vriend G. 2002. Increasing the precision of comparative models with YASARA NOVA - a self-parameterizing force field. Proteins-Structure Function and Genetics 47(3): 393-402.

Kulkarni M, Phalke S. 2009. Evaluating variability of root size system and its constitutive traits in hot pepper (Capsicum annum L.) under water stress. Scientia Horticulturae 120(2): 159-166.

Kumar S, Stecher G, Tamura K. 2016. MEGA7: Molecular Evolutionary Genetics Analysis Version 7.0 for Bigger Datasets. Molecular Biology and Evolution 33(7): 1870-1874.

Kurunc A, Unlukara A, Cemek B. 2011. Salinity and drought affect yield response of bell 
pepper similarly. Acta Agriculturae Scandinavica Section B-Soil and Plant Science 61(6): 514-522.

Leon J, Royo J, Vancanneyt G, Sanz C, Silkowski H, Griffiths G, Sanchez-Serrano JJ. 2002. Lipoxygenase $\mathrm{H} 1$ gene silencing reveals a specific role in supplying fatty acid hydroperoxides for aliphatic aldehyde production. Journal of Biological Chemistry 277(1): 416-423.

Liu SQ, Liu XH, Jiang LW. 2011. Genome-wide identification, phylogeny and expression analysis of the lipoxygenase gene family in cucumber. Genetics and Molecular Research 10(4): 2613-2636.

Livak KJ, Schmittgen TD. 2001. Analysis of relative gene expression data using real-time quantitative PCR and the 2(T)(-Delta Delta C) method. Methods 25(4): 402-408.

Losvik A, Beste L, Glinwood R, Ivarson E, Stephens J, Zhu LH, Jonsson L. 2017. Overexpression and down-regulation of barley lipoxygenase LOX2.2 affects jasmonate-regulated genes and aphid fecundity. International Journal of Molecular Sciences 18(12): 2765; doi:2710.3390/ijms18122765.

Mariutto M, Duby F, Adam A, Bureau C, Fauconnier ML, Ongena M, Thonart P, Dommes J. 2011. The elicitation of a systemic resistance by Pseudomonas putida BTP1 in tomato involves the stimulation of two lipoxygenase isoforms. BMC Plant Biology 11: 29.

Melan MA, Dong XN, Endara ME, Davis KR, Ausubel FM, Peterman TK. 1993. An Arabidopsis thaliana lipoxygenase gene can be induced by pathogens, abscisic-Acid, and methyl jasmonate. Plant Physiology 101(2): 441-450.

Nicholas KB, Nicholas HB, Deerfield DW. 1997. GeneDoc: analysis and visualization of genetic variation. EMBNEW. NEWS 4: 14.

Padilla MN, Hernandez ML, Sanz C, Martinez-Rivas JM. 2009. Functional characterization of two 13-lipoxygenase genes from olive fruit in relation to the biosynthesis of volatile compounds of virgin olive oil. Journal of Agricultural and Food Chemistry 57(19): 9097-9107.

Padilla MN, Hernandez ML, Sanz C, Martinez-Rivas JM. 2012. Molecular cloning, functional characterization and transcriptional regulation of a 9-lipoxygenase gene from olive. Phytochemistry 74: 58-68.

Pakdeevaraporn P, Wasee S, Taylor PWJ, Mongkolporn O. 2005. Inheritance of resistance to anthracnose caused by Colletotrichum capsici in Capsicum. Plant Breeding 124(2): 206-208.

Podolyan A, White J, Jordan B, Winefield C. 2010. Identification of the lipoxygenase gene family from Vitis vinifera and biochemical characterisation of two 13-lipoxygenases expressed in grape berries of Sauvignon Blanc. Functional Plant Biology 37(8): 767-784.

Porta H, Rocha-Sosa M. 2002. Plant lipoxygenases. Physiological and molecular features. Plant Physiology 130(1): 15-21.

Qin C, Yu CS, Shen YO, Fang XD, Chen L, Min JM, Cheng JW, Zhao SC, Xu M, Luo Y, Yang YL, Wu ZM, Mao LK, Wu HY, Ling-Hu CY, Zhou HK, Lin HJ, Gonzalez-Morales S, Trejo-Saavedra DL, Tian H, Tang X, Zhao MJ, Huang ZY, Zhou AW, Yao XM, Cui JJ, Li WQ, Chen Z, Feng YQ, Niu YC, Bi SM, Yang XW, Li WP, Cai HM, Luo XR, Montes-Hernandez S, Leyva-Gonzalez MA, Xiong ZQ, He XJ, Bai LJ, Tan S, Tang XQ, Liu D, Liu JW, Zhang SX, Chen MS, Zhang L, Zhang L, Zhang YC, Liao WQ, Zhang Y, Wang M, Lv XD, Wen B, Liu HJ, Luan HM, Zhang YG, Yang S, Wang XD, Xu JH, Li XQ, Li SC, Wang JY, Palloix A, Bosland PW, Li YR, Krogh A, Rivera-Bustamante RF, Herrera-Estrella L, Yin Y, Yu JP, Hu KL, Zhang ZM. 2014. Whole-genome sequencing of cultivated and wild peppers provides insights into Capsicum domestication and specialization. Proceedings of the National Academy of Sciences of the United States of America 
111(14): 5135-5140.

Roy A, Kucukural A, Zhang Y. 2010. I-TASSER: a unified platform for automated protein structure and function prediction. Nature Protocols 5(4): 725-738.

Royo J, Vancanneyt G, Perez AG, Sanz C, Stormann K, Rosahl S, SanchezSerrano JJ. 1996. Characterization of three potato lipoxygenases with distinct enzymatic activities and different organ-specific and wound-regulated expression patterns. Journal of Biological Chemistry 271(35): 21012-21019.

Sarde S, Bouwmeester K, Venegas-Molina J, David A, Boland W, Dicke M. 2018. Involvement of sweet pepper CaLOX2 in jasmonate-dependent induced defence against Western flower thrips. Journal of Integrative Plant Biology: 10.1111/jipb.12742.

Shen JY, Tieman D, Jones JB, Taylor MG, Schmelz E, Huffaker A, Bies D, Chen KS, Klee HJ. 2014. A 13-lipoxygenase, TomloxC, is essential for synthesis of C5 flavour volatiles in tomato. Journal of Experimental Botany 65(2): 419-428.

Shibata D, Axelrod B. 1995. Plant lipoxygenases. Journal of Lipid Mediators and Cell Signalling 12(2-3): 213-228.

Shin JH, Van K, Kim DH, Do Kim K, Jang YE, Choi BS, Kim MY, Lee SH. 2008. The lipoxygenase gene family: a genomic fossil of shared polyploidy between Glycine max and Medicago truncatula. BMC Plant Biology 8: 133.

Shipp JL, Hao X, Papadopoulos AP, Binns MR. 1998. Impact of western flower thrips (Thysanoptera : Thripidae) on growth, photosynthesis and productivity of greenhouse sweet pepper. Scientia Horticulturae 72(2): 87-102.

Sloane DL, Leung R, Craik CS, Sigal E. 1991. A primary determinant for lipoxygenase positional specificity. Nature 354(6349): 149-152.

Steczko J, Donoho GP, Clemens JC, Dixon JE, Axelrod B. 1992. Conserved histidine-residues in Soybean lipoxygenase - Functional consequences of their replacement. Biochemistry 31(16): 4053-4057.

Steenbergen M, Abd-el-Haliem A, Bleeker P, Dicke M, Escobar-Bravo R, Cheng G, Haring MA, Kant MR, Kappers I, Klinkhamer PGL, Leiss KA, Legarrea S, Macel M, Mouden S, Pieterse CMJ, Sarde SJ, Schuurink RC, De Vos M, Van Wees SCM, Broekgaarden C. 2018. Thrips advisor: exploiting thrips-induced defences to combat pests on crops. Journal of Experimental Botany 69(8): 1837-1848.

ul Hassan MN, Zainal Z, Ismail I. 2015. Green leaf volatiles: biosynthesis, biological functions and their applications in biotechnology. Plant Biotechnology Journal 13(6): 727-739.

Umate P. 2011. Genome-wide analysis of lipoxygenase gene family in Arabidopsis and rice. Plant Signaling and Behavior 6(3): 335-338.

Vandesompele J, De Preter K, Pattyn F, Poppe B, Van Roy N, De Paepe A, Speleman F. 2002. Accurate normalization of real-time quantitative RT-PCR data by geometric averaging of multiple internal control genes. Genome Biology 3(7): RESEARCH0034.

VanDoorn A, Kallenbach M, Borquez AA, Baldwin IT, Bonaventure G. 2010. Rapid modification of the insect elicitor $\mathrm{N}$-linolenoyl-glutamate via a lipoxygenase-mediated mechanism on Nicotiana attenuata leaves. BMC Plant Biology 10: 164.

Vellosillo T, Martinez M, Lopez MA, Vicente J, Cascon T, Dolan L, Hamberg M, Castresana C. 2007. Oxylipins produced by the 9-lipoxygenase pathway in Arabidopsis regulate lateral root development and defense responses through a specific signaling cascade. Plant Cell 19(3): 831-846.

Vogt J, Schiller D, Ulrich D, Schwab W, Dunemann F. 2013. Identification of lipoxygenase 
( $L O X)$ genes putatively involved in fruit flavour formation in apple (Malus $x$ domestica). Tree Genetics \& Genomes 9(6): 1493-1511.

Yan LH, Zhai QZ, Wei JN, Li SY, Wang B, Huang TT, Du MM, Sun JQ, Kang L, Li CB, Li CY. 2013. Role of Tomato lipoxygenase $D$ in wound-induced jasmonate biosynthesis and plant immunity to insect herbivores. PLoS Genetics 9(12): e1003964.

Yates A, Akanni W, Amode MR, Barrell D, Billis K, Carvalho-Silva D, Cummins C, Clapham P, Fitzgerald S, Gil L, Giron CG, Gordon L, Hourlier T, Hunt SE, Janacek SH, Johnson N, Juettemann T, Keenan S, Lavidas I, Martin FJ, Maurel T, McLaren W, Murphy DN, Nag R, Nuhn M, Parker A, Patricio M, Pignatelli M, Rahtz M, Riat HS, Sheppard D, Taylor K, Thormann A, Vullo A, Wilder SP, Zadissa A, Birney E, Harrow J, Muffato M, Perry E, Ruffier M, Spudich G, Trevanion SJ, Cunningham F, Aken BL, Zerbino DR, Flicek P. 2016. Ensembl 2016. Nucleic Acids Research 44(D1): D710-D716.

Zhang B, Chen KS, Bowen J, Allan A, Espley R, Karunairetnam S, Ferguson I. 2006. Differential expression within the LOX gene family in ripening kiwifruit. Journal of Experimental Botany 57(14): 3825-3836.

Zhang B, Yin XR, Li X, Yang SL, Ferguson IB, Chen KS. 2009. Lipoxygenase gene expression in ripening kiwifruit in relation to ethylene and aroma production. Journal of Agricultural and Food Chemistry 57(7): 2875-2881.

Zhang C, Jin YZ, Liu JY, Tang YF, Cao SX, Qi HY. 2014. The phylogeny and expression profiles of the lipoxygenase ( $L O X$ ) family genes in the melon (Cucumis melo L.) genome. Scientia Horticulturae 170: 94-102. 




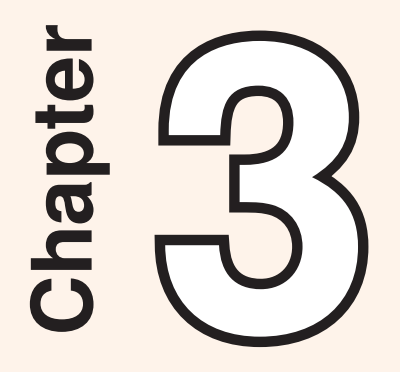

\title{
Involvement of sweet pepper CaLOX2 in jasmon- ate-dependent induced defence against Western flower thrips
}

\author{
SJ. Sarde, K. Bouwmeester, J. Venegas-Molina, A. David, \\ W. Boland and M. Dicke
}

Published in Journal of Integrative Plant Biology (2018), doi:10.1111/jipb.12742 


\section{Abstract}

Insect herbivory can seriously hinder plant performance and reduce crop yield. Thrips are minute cell-content-feeding insects that are important vectors of viral plant pathogens, and are serious crop pests. We investigated the role of a sweet pepper (Capsicum annuum) lipoxygenase gene, CaLOX2, in the defense of pepper plants against Western flower thrips (Frankliniella occidentalis). This was done through a combination of in-silico, transcriptional, behavioral and chemical analyses. Our data show that CaLOX2 is involved in jasmonic acid (JA) biosynthesis and mediates plant resistance. Expression of the JA-related marker genes, CaLOX2 and CaPIN II, was induced by thrips feeding. Silencing of CaLOX2 in pepper plants through virus-induced gene silencing (VIGS) resulted in low levels of CaLOX2 transcripts, as well as significant reduction in the accumulation of $\mathrm{JA}$, and its derivatives, upon thrips feeding compared to control plants. CaLOX2-silenced pepper plants exhibited enhanced susceptibility to thrips. This indicates that CaLOX2 mediates JA-dependent signalling, resulting in defense against thrips. Furthermore, exogenous application of JA to pepper plants increased plant resistance to thrips, constrained thrips population development and made plants less attractive to thrips. Thus, a multidisciplinary approach shows that an intact lipoxygenase pathway mediates various components of sweet pepper defense against $F$. occidentalis.

Key words: Pepper, CaLOX2, defence, phylogeny, synteny, gene expression, VIGS, gene silencing, thrips performance 


\section{Introduction}

In nature, land plants and insects have coexisted for more than 400 million years. Plants perceive herbivorous insects by the specific pattern of tissue disruption and/ or chemical cues originating from insects (Bonaventure, 2012; Heidel-Fischer et al., 2014). Plants have three main signal-transduction pathways, each involving a major plant hormone; i.e., jasmonic acid (JA), salicyclic acid (SA) and ethylene (ET), underlying induced defence against attackers such as herbivorous insects (Pieterse et al., 2012; Stam et al., 2014). JA is well-known to be a key regulator of defense, induced by chewing insects and cell-content feeders like thrips (De Vos et al., 2005; Abe et al., 2008; Abe et al., 2009; Pieterse et al., 2012), whereas SA is known to mediate induced plant defense responses against phloem feeders (Zhu-Salzman et al., 2004; Walling, 2008; Pieterse et al., 2012; Tzin et al., 2015). The three major signaling pathways may exhibit crosstalk. For instance, JA and SA usually act antagonistically, but are also reported to act synergistically, or additively (Pieterse et al., 2012; Thaler et al., 2012).

The Western flower thrips, Frankliniella occidentalis (Pergande) (Thysanoptera: Thripidae) is a polyphagous and economically important pest. Thrips insert their stylets into plant tissues and ingest cell contents, resulting in a silvery appearance of the damaged area (Steenbergen et al., 2018). They feed on almost all aboveground organs of pepper plants and are considered the most devastating pest in greenhouses, worldwide. Their feeding affects leaf size and photosynthetic capacity, which eventually reduces plant growth and productivity (Steiner, 1990; Welter et al., 1990; Shipp et al., 1998). Thrips often aggregate in narrow crevices on the plants, such as in the flowers, developing fruits, foliage and buds, making them difficult to control. Moreover, they also cause indirect damage by transmitting tospoviruses, such as Tomato spotted wilt virus (TSWV) (Maris et al., 2003). Thus, the development of novel approaches to control thrips damage by using knowledge on the molecular mechanisms of plant responses is important.

Various studies have addressed induced plant defenses against leaf-chewing and phloem feeding herbivores (Walling, 2000; Bonaventure, 2012; Heidel-Fischer et al., 2014; Stam et al., 2014; Zust \& Agrawal, 2016), whereas much less is known about plant responses to cell-content feeding thrips. To our knowledge, few studies have reported on the role of $\mathrm{JA}$ in regulating induced plant defense responses against thrips feeding. In Arabidopsis and Chinese cabbage (Brassica rapa subsp. pekinensis), JA is involved in defense against thrips (Abe et al., 2008; Abe et al., 2009). In tomato, the JA-signaling mutant, Defenceless 1 (Def1), exhibits enhanced susceptibility to thrips feeding (Li et al., 2002; Escobar-Bravo et al., 2017) and a decline in thrips abundance was observed in tomato upon JA application in field conditions (Thaler et al., 2001). 
Likewise, cotton and soybean plants also show increased resistance against thrips upon exogenous JA or MeJA application (Omer et al., 2001; Selig et al., 2016). In Arabidopsis, a total of 199 genes were differentially expressed (up and down regulated) upon thrips feeding, among which 138 (69\%) were JA-responsive genes (De Vos et al., 2005). However, in pepper plants, little is known about the mechanisms underlying defense against insect herbivores.

Lipoxygenases (LOXs) are enzymes encoded by a multi-gene family functioning in different plant developmental and defense processes (Brash, 1999). They are wellknown to oxygenate fatty acids. In plants, the main classes are 9-lipoxygenases and 13-lipoxygenases that oxygenate lipids at the $9^{\text {th }}$ and $13^{\text {th }}$ carbon atom, respectively (Feussner \& Wasternack, 2002). In JA biosynthesis, the first oxygenation step of linolenic acid is performed by a 13-lipoxygenase (Brash, 1999; Feussner \& Wasternack, 2002). Disruption of this 13-LOX has been shown to suppress the JA pathway in several plant species, resulting in enhanced susceptibility to insect herbivory.

In Nicotiana attenuata silencing through antisense expression of NaLOX3, involved in JA synthesis, suppressed JA synthesis and enhanced herbivore performance (Halitschke \& Baldwin, 2003). Similarly, in tomato, overexpression of TomLOXD elevated levels of JA and resistance to a caterpillar species (Yan et al., 2013). The 13-LOXs have also been identified in Arabidopsis (Bell et al., 1995), potato (Royo et al., 1996), rice (Zhou et al., 2009) and Asian ginseng (Rahimi et al., 2017). In pepper, a 9-LOX, CaLOX1, has been identified and reported to be involved in defense and cell-death responses to pathogens (Hwang \& Hwang, 2010). However, to date, no 13-type LOX has been characterized in pepper (Capsicum annuum).

The main objective of the present study was to identify and characterize a 13-LOX gene specifically involved in JA biosynthesis and subsequently elucidate its role in JA-regulated defense of a non-model plant, sweet pepper, against thrips. We performed in-silico analysis to identify the pepper 13-lipoxygenase gene that is potentially involved in JA-biosynthesis (termed CaLOX2) and evaluated performance and preference of thrips on plants subjected to exogenous application of JA and silencing of the CaLOX2 gene by virus-induced gene silencing (VIGS). For this, we initially investigated the induction of JA- and SA-regulated genes, upon thrips feeding, and long-term effects of exogenous JA application on thrips population size, preference of thrips between JA-treated and non-treated plants, and plant resistance. Furthermore, the consequences of silencing CaLOX2 through VIGS for production of down-stream JA-related phytohormones and performance and preference of thrips were studied. Thus, this study assesses the role of CaLOX2 in jasmonic acid-dependent signaling underlying the defense response to thrips feeding in the non-model plant sweet pepper. 


\section{Materials and methods}

\section{Plant material and thrips rearing}

Sweet pepper (Capsicum annuum; Mandy variety) (Rijk Zwaan, De Lier, The Netherlands) plants were grown in pots of $12 \mathrm{~cm}$ diameter in a greenhouse at $23-25^{\circ} \mathrm{C}$ with a $16 \mathrm{~L}: 8 \mathrm{D}$ photoperiod and $70 \pm 10 \%$ relative humidity. Four-week-old plants were used in the experiments. One day before the experiments, plants were transferred to a climate chamber with controlled conditions $\left(24 \pm 1^{\circ} \mathrm{C}, 70 \pm 10 \%\right.$ relative humidity, 16L:8D photoperiod and light intensity of $70 \mu \mathrm{mol}$ photons $\left.\mathrm{m}^{-2} \mathrm{~s}^{-1}\right)$. Western flower thrips (WFT; Frankliniella occidentalis) were reared on bean pods (Phaseolus vulgaris $)$ in a climate-controlled cabinet $\left(25 \pm 2{ }^{\circ} \mathrm{C}, 70 \pm 10 \%\right.$ relative humidity, L16:8D photoperiod).

\section{Sequence retrieval, homology search and domain analysis}

Tomato (Solanum lycopersicum) LOX protein sequences were retrieved from the Ensembl Plants genome browser (http://plants.ensembl.org/index.html). Sequences of LOX proteins from other plant species, i.e. Solanum tuberosum, Nicotiana attenuata, Brassica napus, and Arabidopsis thaliana were downloaded from the NCBI repository. The tomato LOXD (Solyc03g122340) protein sequence was used to identify CaLOX2 in pepper. All LOX proteins were subjected to Pfam (v27.0) domain analysis (Finn et al., 2014) using CLC Main Workbench (Version 7.6.4). The pepper genome/ proteome subjected to analysis was derived from Capsicum annuum L. Zunla-1 (Qin et al., 2014).

\section{Phylogenetic and synteny analysis}

Full-length protein sequences were used for alignment with the MUSCLE tool (https:// www.ebi.ac.uk/Tools/msa/muscle/) using the default parameters. The obtained alignment was used for construction of a Maximum Likelihood phylogenetic tree with 1000 bootstrap replicates using MEGA5.1 (Tamura et al., 2011). Synteny analysis of solanaceous LOX genes, specifically involved in the octadecanoid pathway, was performed using the Ensembl Plants genome browser (Yates et al., 2016), Mapviewer from NCBI (https://www.ncbi.nlm.nih.gov/mapview/) and the Gene database from NCBI (https://www.ncbi.nlm.nih.gov/gene).

\section{Plasmid construction}

The pTRV ( Tobacco rattle virus)-based VIGS protocol (Wang et al., 2013; Senthil-Kumar \& Mysore, 2014) was used to generate CaLOX2-silenced (TRV:CaLOX2) pepper plants. For this purpose, the unique coding gene fragment of the Lipoxygenase-2 gene of Capsicum annuum (CaLOX2, Capana03g000103) was selected and ampli- 
fied by PCR. The specificity of the selected sequence was checked via the VIGS tool on the Sol Genomics Network (http://vigs.solgenomics.net/). A gene fragment of 282 bp (Supplementary file 1) was cloned into the TRV2-vector and subsequently transformed into Agrobacterium tumefaciens GV3101 strain via electroporation. Presence of the CaLOX2 fragment in the TRV2-vector was verified by restriction digestion and sequencing.

\section{Agro-infiltration and TRV-mediated silencing assays}

Agrobacterium tumefaciens GV3101 strains carrying vectors were grown overnight at $28^{\circ} \mathrm{C}$ in YEB (Yeast Extract Broth) media with appropriate antibiotics (rifampicin 25 $\mu \mathrm{g} / \mathrm{ml}$, kanamycin $50 \mu \mathrm{g} / \mathrm{ml}$ ). A. tumefaciens cells were centrifuged and resuspended in induction medium containing rifampicin $(25 \mu \mathrm{g} / \mathrm{ml})$, kanamycin $(50 \mu \mathrm{g} / \mathrm{ml})$ and acetosyringone $(50 \mu \mathrm{g} / \mathrm{ml})$ antibiotics for 3-4 $\mathrm{h}$, and thereafter for $1 \mathrm{~h}$ in infiltration medium with acetosyringone $(150 \mu \mathrm{g} / \mathrm{ml})$. The composition of induction and infiltration medium is provided in Supplementary file 1. Agrobacterium tumefaciens GV3101 cultures carrying pTRV1 and pTRV2:GUS or pTRV2:CaLOX2 or pTRV2:NaPDS were mixed at a ratio of $1: 1$ to a final $O_{600}$ of 1.0 and syringe-infiltrated into cotyledons of two-weekold pepper seedlings (Wang et al., 2013). Due to high sequence similarity between NaPDS and CaPDS, we used pTRV2:NaPDS construct to monitor the proliferation of silencing in pepper plants. The plants prior and post Agrobacterium-infiltration were kept in a greenhouse at $23-25^{\circ} \mathrm{C}, 70 \pm 10 \%$ relative humidity and $16 \mathrm{~L}: 8 \mathrm{D}$ photoperiod. Efficiency of CaLOX2 silencing was validated by RT-qPCR.

\section{No-choice and two-choice feeding bioassays post VIGS}

To determine the role of CaLOX2 in sweet pepper resistance to thrips, the youngest fully-grown true leaves of three-week-old Agrobacterium-infiltrated plants were used for no-choice and two-choice thrips bioassays. A no-choice experiment was conducted by using one detached leaf of pTRV2:GUS or pTRV2:CaLOX2 plants. Each detached leaf was placed in a separate Petri dish (140-mm $\times 20-\mathrm{mm})$. The petiole was inserted in a $1 \%$ agar solution. Five adult female thrips were placed on each leaf in the Petri dish for 6 or 24-h (with 14 replicates) and thrips feeding-associated damage was recorded at these time points. In addition, infested leaves were sampled for qRT-PCR and phytohormone analyses. In two-choice assays, one leaf each of a TRV2:CaLOX2 and a TRV2:GUS plant were placed adjacent to each other in a Petri dish (with 14 replicates). Five adult female thrips were placed in the center of the Petri dish, equidistant from the leaves, and were allowed to feed on the leaves for two 2-3 d. The area of feeding scars (on both abaxial and adaxial leaf sides) was measured using a light microscope and a transparent grid sheet. 


\section{JA treatment and bioassays}

To test the effect of the induction of JA signaling on sweet pepper resistance to thrips, four-week-old sweet pepper plants were sprayed with $100 \mu \mathrm{M} \mathrm{JA}$, or mock-treated with water, both mixed with $0.1 \%$ of Tween 20 (detailed protocol in Supplementary file 1). The treatment was conducted one day before the bioassay. The experiments (non-choice and choice assays) were performed in a climate chamber under controlled conditions $\left(24 \pm 1^{\circ} \mathrm{C}, 70 \pm 10 \%\right.$ relative humidity, $70 \mu \mathrm{mol}$ photons $\mathrm{m}^{-2} \mathrm{~s}^{-1}$ light intensity and 16L:8D photoperiod). For a non-choice feeding bioassay, 25 female adult thrips were placed on each JA-treated and non-treated whole pepper plants confined in transparent plastic cages covered with mesh on top. After $14 \mathrm{~d}$, the number of their offspring (first and second-instar larvae) and feeding damage on plants was assessed. The experiment was executed twice, one for assessment of offspring number (with 10 replicate plants) and one for assessment of feeding damage (with 12 replicate plants). In preference (choice) assays (with 11 replicates), a JA-treated and a mock-treated plant were positioned on either side of a transparent plastic box (height: $310 \mathrm{~mm}$, width: $440 \mathrm{~mm}$, length: $710 \mathrm{~mm}$ ) and 50 adult female thrips were placed halfway between them. The boxes were incubated in a climate chamber with controlled conditions $\left(24 \pm 1^{\circ} \mathrm{C}, 70 \pm 10 \%\right.$ relative humidity, $16 \mathrm{~L}: 8 \mathrm{D}$ photoperiod). Two $d$ later, the numbers of thrips on each plant were recorded.

\section{RNA isolation and qRT-PCR}

To test the effect of thrips feeding on JA and SA-associated defense genes, five second instar thrips larvae (L2) were introduced into a clip cage and allowed to feed on one of the first two true leaves of four-week-old sweet pepper plants. Plants with clip cages without thrips served as controls. At $0,5,10$ and $24 \mathrm{~h}$ after infestation, leaves were sampled for gene expression analyses of JA- and SA-associated marker genes. For each treatment and time point, 4-5 biological replicates were analyzed, each replicate consisting of one individual plant. RNA extraction was executed using the Bioline kit, in accordance to the manufacturer's protocol. cDNA was synthesized from $1 \mu \mathrm{g}$ of total RNA with iScript cDNA synthesis kit (Bio-Rad). For qPCR analysis, a 25 $\mu \mathrm{l}$ reaction mixture, containing $1 \mu \mathrm{l}(10 \mu \mathrm{M})$ of forward and reverse primers, $12.5 \mu \mathrm{l}$ of SYBR Green Supermix (Bio-Rad) and $5 \mu \mathrm{l}$ cDNA, was used. The reference gene, CaACTIN, was used as normalizer for determining the relative expression of JA-related genes (CaLOX2; Capsicum annuum Lipoxygenase 2 and CaPIN II; Capsicum annuum Protease Inhibitor II) and an SA-dependent gene (CaPR1; Capsicum annuum Pathogenesis Related 1). The following PCR conditions were used: 3 min at $95^{\circ} \mathrm{C}$, followed by 40 cycles of $15 \mathrm{~s}$ at $95^{\circ} \mathrm{C}$, and $45 \mathrm{~s}$ at $60^{\circ} \mathrm{C}$. At the end of each qPCR, the melting curve of each gene was recorded. All primers used for qPCR pre and post VIGS are presented in Supplementary file 1. Relative gene expression was analyzed 
using the geometric mean of threshold cycle (Ct) values (Vandesompele et al., 2002) for the reference gene CaACTIN with the 2- ${ }^{\Delta C \mathrm{Ct}}$ method (Livak \& Schmittgen, 2001).

\section{Hormone quantification}

Leaf samples (100 mg each) from pTRV2:GUS and pTRV2:CaLOX2 plants were flash-frozen in liquid nitrogen and stored at $-80^{\circ} \mathrm{C}$. High-performance liquid chromatography-mass spectrometry (HPLC-MS/MS) was used to quantify JA (jasmonic acid), JA-lle (jasmonic acid isoleucine) and OPDA (12-oxo-phytodienoic acid) content, according to the method described in Trapp et al. (2014).

\section{Statistical analysis}

Data on gene expression and hormone content were log-transformed prior to statistical analyses. The data on gene expression, thrips feeding, gene silencing efficiency post VIGS and hormonal quantification were all subjected to a Student's $t$-test. Moreover, thrips two-choice preference data were expressed as the proportion of thrips detected on either treatment. The data were analyzed by a t-test within each treatment to determine if the proportion of thrips significantly differed from 0.5 , as previously described (Grostal and Dicke (1999). The data of the two-choice feeding experiment post VIGS treatment were analyzed by a paired t-test. These analyses were performed using software IBM SPSS Statistics for Windows, version 23 (IBM Corp., Armonk, N.Y., USA). Biological replicates used for statistics each consist of one individual plant.

\section{Results}

\section{Identification of CaLOX2 in pepper}

To identify the LOX2 homolog in pepper, all sequences of tomato LOX proteins were used as queries in blast searches for a genome-wide search against the protein database of Capsicum annuum L. Zunla-1. This resulted in the identification of several LOX proteins in pepper (Sarde et al., 2018). These LOX homologs were further scanned for the presence of PLAT/LH2 and LOX domains that are hallmarks of the lipoxygenase gene family (Chen et al., 2015), using the Pfam database. To narrow down the selection towards the specific LOX protein induced upon herbivory or wounding in sweet pepper, the closest homolog of the tomato LOXD gene, well-known to have a similar function (Yan et al., 2013), was selected from pepper (CaLOX2, Capana03g000103) and further subjected to phylogenetic and synteny analysis. 

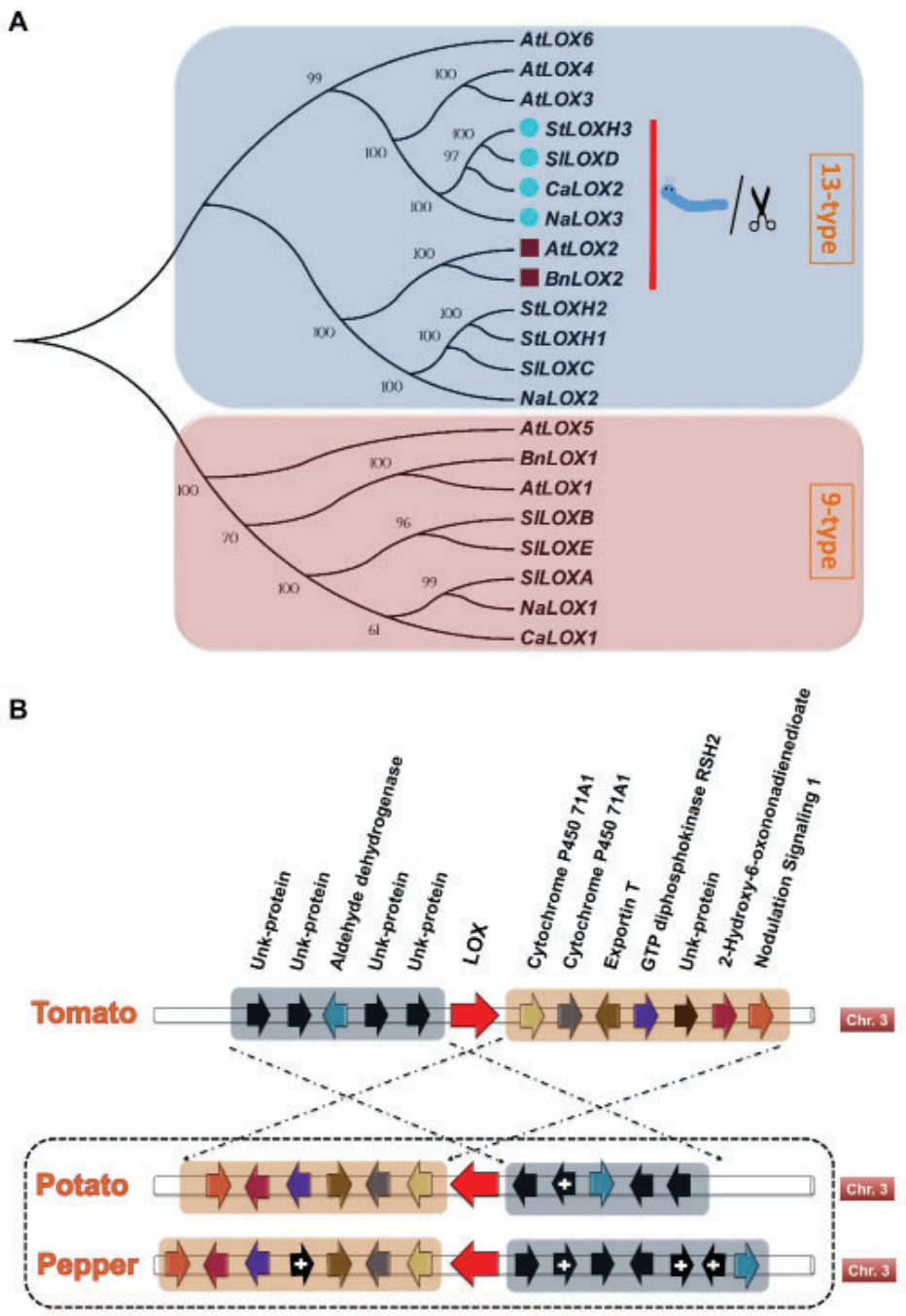

Figure 1. Phylogenetic and synteny analyses of lipoxygenases. (A) Phylogenetic relationship of lipoxygenases of six species from the Brassicaceae and Solanaceae families. The tree was generated by MEGA 5.1 using the Maximum Likelihood method with 1000 bootstraps. Accession numbers or Gene IDs of sequences used to construct the phylogenetic tree are as follows. Arabidopsis thaliana: AtLOX1, AAA32827; AtLOX2, AAA32749; AtLOX3, AT1G17420; AtLOX4, AT1G72520; AtLOX5, AT3G22400; AtLOX6, AT1G67560; Brassica napus: BnLOX1, AAO03558, BnLOX2, NP_001303054; Solanum lycopersicum: SILOXC, AAB65766; SILOXD, AAB65767; SILOXB, AAAA53183; SILOXE, AAG21691; SILOXA, AAA53184; Solanum tuberosum: StLOXH2, CAA65268; StLOXH3, CAA65269; StLOX1, AAB67858; Capsicum annuum: CaLOX2, Capana03g000103; CaLOX1, ACO57136; Nicotiana attenuata: NaLOX1, AAP83134; NaLOX2, AAP83137; NaLOX3, AAP83138. (B) Syntenic organization of tomato (SILOXD), potato (StLOXH3) and pepper (CaLOX2) LOX genes. Black arrows with and without "+" sign depict genes that are not identical to synteny of tomato or unknown (uncharacterized) genes, respectively. 
Phylogenetic analysis was conducted to analyze the evolutionary relationship between several LOX proteins across different species of the Brassicaceae and Solanaceae families. The phylogenetic tree (Figure $1 \mathrm{~A}$ ) clearly represents two major clades of 13-type and 9-type LOX proteins. In the 13-type clade, the related pepper LOX2 protein is positioned in the sub-clade of LOX proteins within the Solanaceae family that are known to be involved in JA biosynthesis. Similarly, this sub-clade appears closer to the Brassicaceae sub-clade comprising LOX proteins that have a similar function (Figure 1A), suggesting a role of CaLOX2 in JA biosynthesis.

Furthermore, genomic locations and assessments of syntenic maps offer insights into the conservation of genes across organisms. In the model plant of the Solanaceae family, tomato, the TomLOXD gene, well-known to be induced upon herbivory, is flanked by five genes on one side and seven genes on the other side (Figure 1B). The synteny is similar, but in reverse order with some exceptions of additional, uncharacterized or unknown genes for its counterpart $L O X$ genes in potato $(L O X H 3)$ and pepper (CaLOX2). Moreover, localization of this gene is still intact on Chromosome 3 in pepper, tomato and potato. Taken together the in-silico analysis suggests a possible role of CaLOX2 in the octadecanoid pathway.

\section{JA and SA-related marker genes are up-regulated upon thrips feeding}

We analyzed the expression of JA and SA-related marker genes in sweet pepper in response to thrips feeding in order to determine the role of JA in defense against thrips. Expression of the in-silico identified CaLOX2 gene was induced upon thrips feeding at all three time points sampled (Figure 2A). Similarly, expression of the downstream JA-responsive gene, CaPIN II, was also up-regulated upon thrips feeding (Figure 2B). Moreover, upon thrips infestation the SA-responsive gene, CaPR1, did not show significant induction after $5 \mathrm{~h}$ but was up-regulated after 10 and $24 \mathrm{~h}$ of thrips feeding (Figure 2C).

\section{Exogenous JA application negatively affects thrips feeding and preference}

Because thrips feeding induces the transcription of JA-related genes, we first investigated the effect of exogenous JA application on thrips population build-up and its feeding. For this purpose, twenty-five adult females were introduced onto control and JA-treated plants. After two weeks, the larvae and adults were counted on each plant. The JA-treated plants had significantly fewer larvae and adult thrips compared to control plants (Figure 3A, B). Control plants had 2.8 times more larvae (first and second instars; Figure $3 \mathrm{~A}$ ) and 3.5 times more adults (Figure $3 \mathrm{~B}$ ) than JA-treated plants. This indicates that JA underlies pepper defense against thrips. 


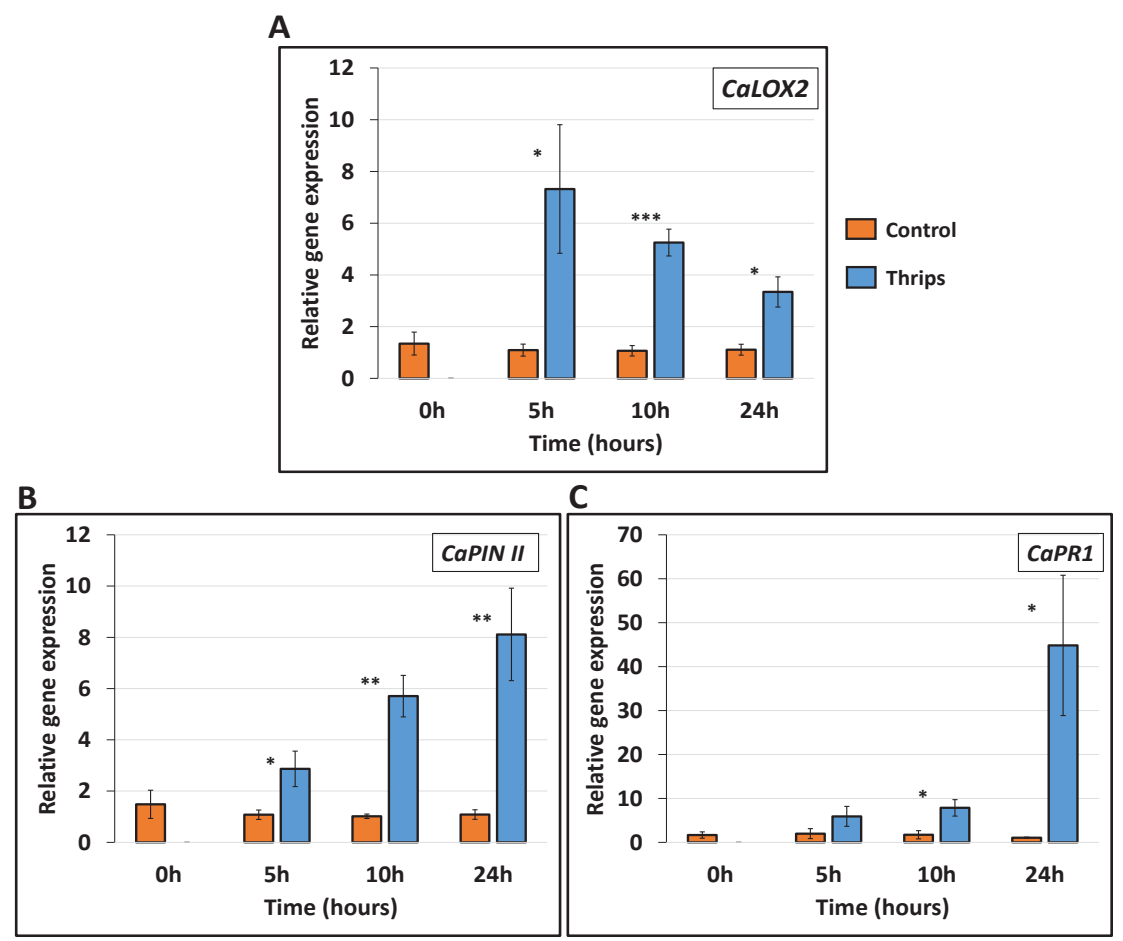

Figure 2. Quantitative RT-PCR (RT-qPCR) of JA and SA-related marker genes in sweet pepper leaves in response to thrips (F. occidentalis) feeding. (A) CaLOX2 and (B) CaPIN II, as marker genes for the JA-pathway and (C) CaPR1, as marker gene for the SA-pathway. Five $2^{\text {nd }}$ instar thrips larvae fed locally (confined in clip cages) on the first true leaf of fourweek-old pepper plants. Empty clip cages were used on control plants. The expression level of each gene was normalized to the expression of the housekeeping gene CaACTIN. Bars represent mean $\pm S E(n=4-5$ biological replicates). Bars marked with asterisks indicate significant differences (Student's t-test), ${ }^{*}$ p-value $<0.05,{ }^{* *} p$-value $<0.01,{ }^{* * *} p$-value $<0.001$.

We subsequently investigated the effect of exogenous JA application on thrips feeding, in the same experimental setup. Significantly more feeding damage was recorded on control plants $\left(450 \mathrm{~mm}^{2}\right)$ compared to JA-treated plants $\left(216 \mathrm{~mm}^{2}\right)$ (Figure $3 \mathrm{C}$ ). These results further support the involvement of $\mathrm{JA}$ in resistance of pepper against thrips.

Finally, we investigated the effect of JA on host plant preference of thrips. To this end, we placed 50 adult females halfway between a control and a JA-treated plant and counted the number of thrips on each plant after 2 days; thrips significantly preferred control plants over JA-treated plants (Figure 3D). 
A

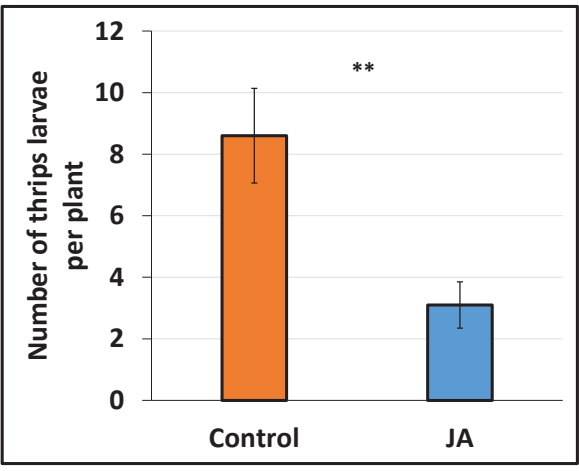

C

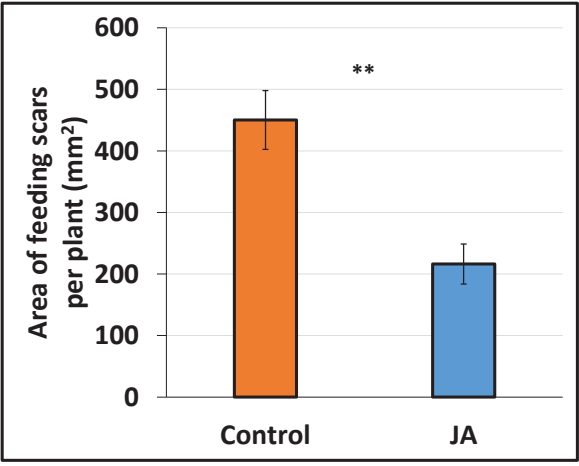

B

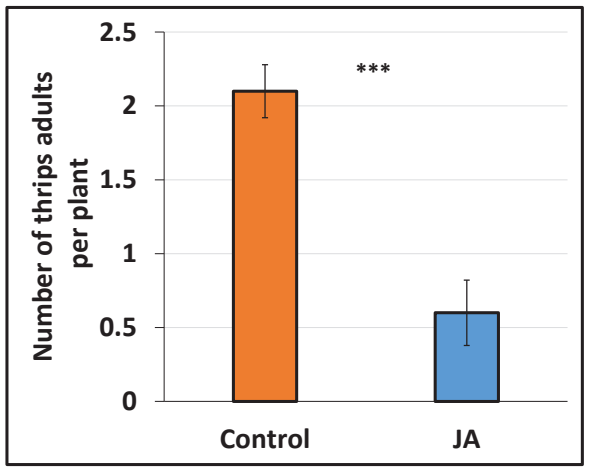

D

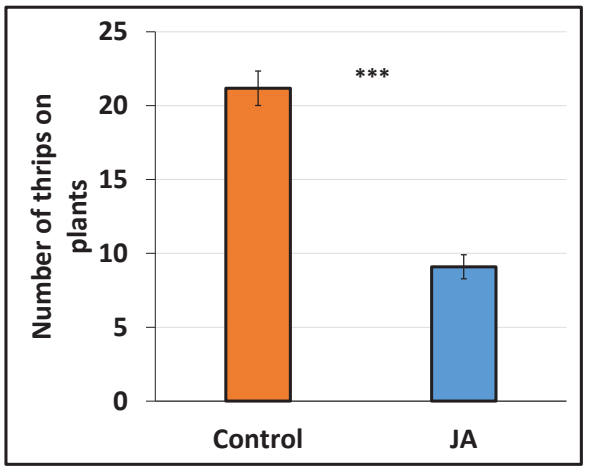

Figure 3. No-choice (thrips population and feeding damage) and choice (preference) tests of Western flower thrips upon exogenous application of JA $(100 \mu \mathrm{M})$. (A, B) Effect of JA application on thrips population and (C) feeding damage. Twenty-five adult females fed on 4-week-old sweet pepper plants for two weeks. Water + Tween20 (control) or $100 \mu \mathrm{M} \mathrm{JA}$ + Tween20 (treatment) was applied 1 day before thrips were introduced on pepper plants. Number of thrips larvae (A), thrips adults (B) and feeding damage (C) in $\mathrm{mm}^{2}$ caused by thrips feeding on plants was assessed after two-weeks. Mean \pm SE based on ten and twelve biological replicates for thrips population and feeding damage, respectively. Asterisks indicate significant differences (Student's t test), ${ }^{* *} p$-value $<0.01$, ${ }^{* * *} p$-value $<0.001$. (D) Effect of exogenous JA on host plant preference of thrips (choice experiment). Mean \pm SE based on eleven biological replicates. Asterisks indicate significant differences (Student's $t$ test, $t=$ 7.598); ${ }^{* * *}$ p-value $<0.001$.

\section{VIGS of CaLOX2 suppresses CaLOX2 expression}

To study the involvement of CaLOX2 in the JA pathway, we silenced CaLOX2 in pepper plants using VIGS. The unique region of $282 \mathrm{bp}$ of the CaLOX2 coding region (Supplementary file 1) was selected, using CLC bio-workbench and its specificity was confirmed using the VIGS tool from the Sol Genomics Network.

To assess the efficiency of VIGS, CaLOX2 transcript levels were quantified using 
qRT-PCR in GUS-vector control (TRV:GUS) and CaLOX2-silenced (TRV:CaLOX2) pepper leaves infested with thrips. CaLOX2 expression was significantly induced, at both time points, in GUS-vector control leaves (Figure S1). In contrast, in CaLOX2-silenced pepper leaves, CaLOX2 transcript levels were significantly decreased compared to GUS-vector control leaves, both experiencing thrips feeding, indicating that CaLOX2 silencing was effective in suppressing CaLOX2 induction (Figure 4A).

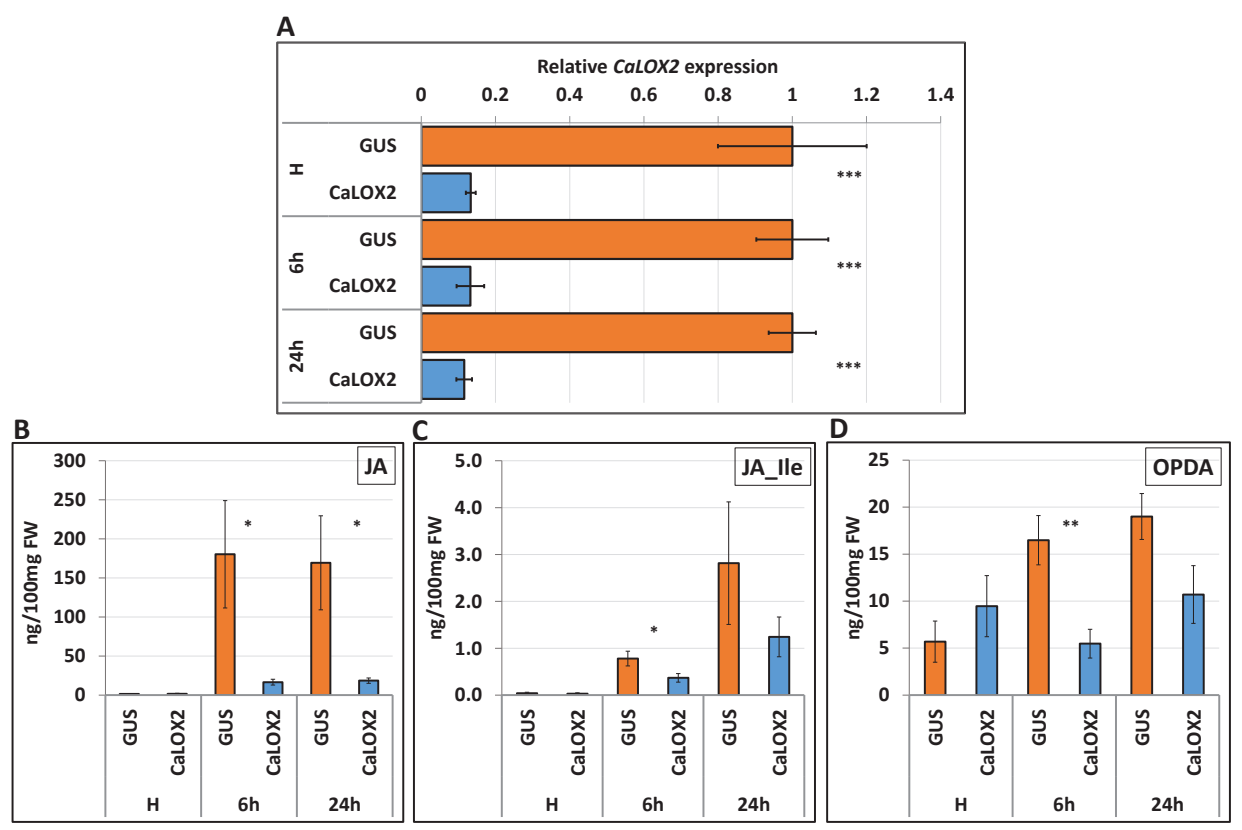

Figure 4. Quantitative RT-PCR (RT-qPCR) of CaLOX2 and phytohormone quantification in GUS-vector control and CaLOX2-silenced leaves infested with western flower thrips for $\mathbf{6} \mathbf{h}$ and $24 \mathrm{~h}$. (A) Silencing efficiency of CaLOX2. H, healthy leaves; GUS, $\beta$-Glucuronidase; CaLOX2, C. annuum Lipoxygenase2. The $C$. annuum actin gene was used for normalization in qPCR. Data are mean \pm SE of 14 biological replicates from two independent experiments. Asterisks indicate significant differences (Student's t test), ${ }^{* * *} p$-value $<0.001$. (B, C, D) Quantification of JA and its derivatives. OPDA, 12-oxo-phytodienoic acid; JA, Jasmonic acid; JA-Ile, Jasmonic acid isoleucine. Five adult females fed on a detached single leaf of a 5 -week-old plant. Data are mean $\pm S E$ of 6 biological replicates. Asterisks indicate significant differences (Student's t test), ${ }^{*}: p$-value $<0.05,{ }^{* *}$ : $p$-value $<0.01,{ }^{* * *}$ : $p$-value $<0.001$.

\section{Silencing of CaLOX2 compromises jasmonate accumulation}

To investigate the effect of CaLOX2 silencing on the accumulation of JA and its derivatives in response to thrips attack, we assessed the levels of phytohormones in leaves with the GUS-vector and CaLOX2-silenced pepper plants infested with thrips (Figure 4). CaLOX2 silencing resulted in lower levels of OPDA, JA and JA-lle. The differences were statistically significant at $6 \mathrm{~h}$ for all three phytohormones and at 24 $\mathrm{h}$ for OPDA and JA. This indicated that CaLOX2 silencing compromised the induc- 
tion of JA, and its derivatives JA-lle, and OPDA in CaLOX2-silenced pepper leaves subjected to thrips feeding (Figure 4B - D). These results support a role of CaLOX2 in the octadecanoid pathway leading to the biosynthesis of JA and JA-lle, as induced upon herbivory.

\section{Suppression of CaLOX2 confers increased susceptibility to thrips feeding}

To assess the consequence of CaLOX2 silencing for thrips feeding and preference, we quantified thrips feeding damage on leaves of GUS-vector control and CaLOX2-silenced pepper plants. This was done in no-choice (Figure 5A) and choice (Figure $5 B)$ tests. Injury resulting from thrips attack was significantly greater on CaLOX2-silenced plants than on non-silenced (GUS) plants, in a no-choice test, at both time points, indicating that a functional CaLOX2 reduces the feeding rate of thrips individuals (Figure 5A). Furthermore, when provided with a choice between silenced leaves (CaLOX2-silenced) and non-silenced (GUS-vector) leaves, thrips clearly preferred to feed on CaLOX2-silenced plants (Figure 5B). Taken together, these results support the role of CaLOX2 in the octadecanoid pathway of induced defense.

A

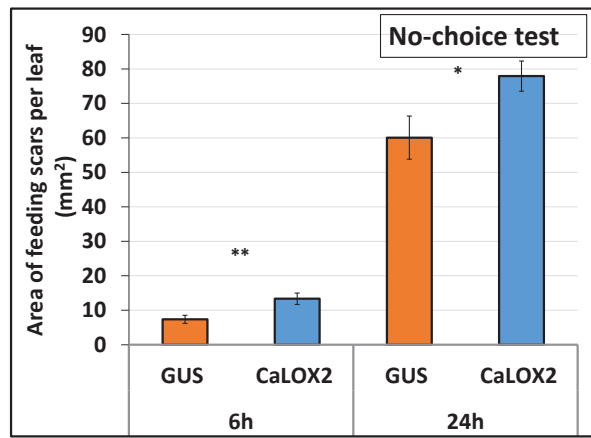

B

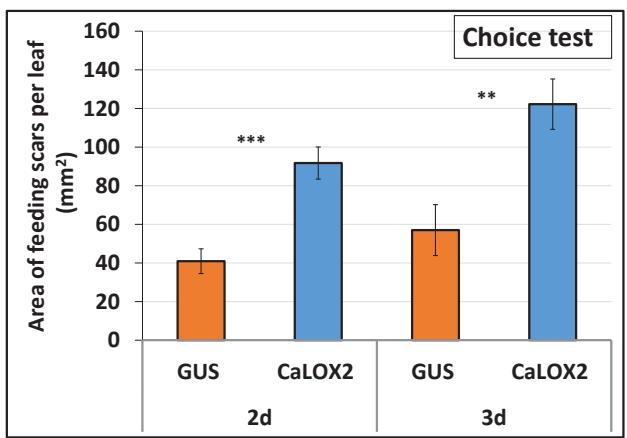

Figure 5. Effect of CaLOX2 silencing by VIGS in sweet pepper plants on thrips feeding in no-choice and thrips preference in choice tests. (A) Area of feeding scars per leaf after 6 and $24 \mathrm{~h}$ of thrips feeding (5 female thrips adults per leaf) in no-choice assay. Mean $\pm \mathrm{SE}$, based on fourteen biological replicates from two independent experiments. Asterisks indicate significant differences (Student's t test), ${ }^{*} p$-value $<0.05,{ }^{* *} p$-value $<0.01$. (B) Area of feeding scars per leaf after 2 and $3 \mathrm{~d}$ of infestation by 5 female thrips adults in choice assay. Mean $\pm \mathrm{SE}$, based on fourteen biological replicates from two independent experiments. Asterisks indicate significant differences (paired t-test), ${ }^{* *} p$-value $<0.01,{ }^{* * *} p$-value $<0.001$.

\section{Discussion}

Through a combination of an in-silico analysis and transcriptional, behavioral and chemical analyses, we show that CaLOX2 (Capana03g000103) is activated by thrips feeding, and involved in the octadecanoid pathway, leading to the phytohormone JA 
and its active conjugate JA-lle, resulting in induced defense against thrips in sweet pepper. Although jasmonates have been reported as important players underlying induced plant defense against various herbivorous insect species (Wasternack, 2007; Howe \& Jander, 2008), most research has focused on defenses against leaf-chewing or phloem-feeding herbivores (De Vos et al., 2005; Bonaventure, 2012; Heidel-Fischer et al., 2014; Stam et al., 2014; Zust \& Agrawal, 2016).

By contrast, relatively little is known about induced plant defenses in response to cell-content feeders like thrips. Most knowledge on inducible defense against thrips is available for model plants, such as Arabidopsis thaliana and tomato, for which specific well-characterized mutants are available (Li et al., 2002; Abe et al., 2008; Abe et al., 2009; Abe et al., 2012). Disentangling the molecular mechanisms underlying induced defense against thrips in crops will be valuable in the selection of resistant varieties.

Few studies have reported that jasmonates regulate induced defenses against thrips (Li et al., 2002; Abe et al., 2008; Abe et al., 2009). Due to the relatively limited availability of genetic tools and genomic information, little is known about the biosynthesis of JA and the role of jasmonates in modulating defense responses in non-model plants, such as pepper. Nonetheless, recent knowledge on genomic information and valuable tools like VIGS provides valuable resources to investigate the role of the octadecanoid pathway in defense against thrips feeding. We employed these tools to identify CaLOX2 as being the LOX gene involved in the JA-pathway and resistance of cultivated pepper to $F$. occidentalis.

By in-silico analysis, we identified CaLOX2 as the gene involved in the octadecanoid pathway. CaLOX2 is a 13-type LOX that is phylogenetically close to functionally similar orthologues in tomato (SILOXD), potato (StLOXH3) and tobacco (NaLOX3), which are specifically involved in the biosynthesis of JA (Figure 1A) (Royo et al., 1996; Kessler et al., 2004; Yan et al., 2013). Moreover, chromosomal locations and comparisons of syntenic maps show that flanking genes are conserved, with some exceptions of uncharacterized or additional genes, and that the CaLOX2 gene is positioned on Chromosome number 3 , across selected plants from the Solanaceae family. This supports the evolutionary significance of this gene to the plants and supports a role of CaLOX2 in the octadecanoid pathway, as further reinforced experimentally in our study.

Our findings clearly show that the JA-related genes, CaLOX2 and CaPIN II, are upregulated at all time points upon thrips feeding, as also recorded for respective JA-related genes in Arabidopsis and Chinese cabbage (Brassica rapa subsp. pekinensis) (De Vos et al., 2005; Abe et al., 2008; Abe et al., 2009). Moreover, the up-regulation of $L O X$ genes was also recorded in cabbage (Brassica oleracea L.) and tomato, upon 
feeding by another cell-content feeding herbivore, the spider mite Tetranychus urticae (Li et al., 2002; Zheng et al., 2007). In contrast, no up-regulation of CaPR1 (SA-related) was recorded until 10 and $24 \mathrm{~h}$ after initiation of thrips feeding, similar to what has been recorded for the SA-related genes PR1 and BGL.2 in Arabidopsis (Abe et al., 2008). De Vos et al. (2005) reported that SA levels were elevated after $12 \mathrm{~h}$ of thrips feeding, corresponding with induction of SA-related genes at later time points. Possibly, thrips manipulate plant defense by inducing SA, to stimulate antagonistic crosstalk with the JA pathway to interfere with plant defense (Abe et al., 2012; Stam et al., 2014).

Both exogenous application of JA and silencing of CaLOX2, which interferes with JA induction demonstrate that JA is involved in inducible defense against thrips. JA is also involved in inducible defense against another cell-content feeding herbivore, the spider mite Tetranychus urticae in Lima bean and tomato plants (Dicke et al., 1999; Li et al., 2002; Gols et al., 2003; Ament et al., 2004). Similar induction of resistance against thrips, by the application of exogenous JA, was also recorded in A. thaliana, Brassica rapa and S. lycopersicum (Li et al., 2002; Abe et al., 2008; Abe et al., 2009).

There are several homologs of LOX genes in a wide range of plants (Zhang et al., 2006; Podolyan et al., 2010; Liu et al., 2011; Umate, 2011; Zhang et al., 2014; Chen et al., 2015). They have been thoroughly characterized and reported to be involved in several plant biological processes, such as plant defense, tuber growth, germination of seeds, and fruit ripening (Kolomiets et al., 2001; Bailly et al., 2002; Porta \& Rocha-Sosa, 2002; Barry \& Giovannoni, 2007; Abe et al., 2008).

Usually, there is at least one LOX homolog induced upon herbivory and involved in the biosynthesis of JA (Kessler et al., 2004; Zhou et al., 2009; Allmann et al., 2010; Zheng et al., 2011; Yan et al., 2013). These phenomena have been well studied in model plants, but little is known in non-model plants. In order to study the role of octadecanoid pathway in different biological processes in plants, JA-deficient mutants have been generated. In Arabidopsis, the Coi1-mutant is often used; this mutant is defective in the JA-lle receptor (Xie et al., 1998). In tomato, several mutant lines, including Def-1, Spr-1, and Spr-2, are available (Howe et al., 1996; Howe \& Ryan, 1999; Li et al., 2002).

Nonetheless, the genomic target region of JA-deficient mutants in tomato is still unclear. In rice and tobacco, a specific homolog of LOX was targeted to generate JA-deficient plants through transformation (Kessler et al., 2004; Zhou et al., 2009). Silencing of OsHI-LOX or Na-LOX3 in rice and tobacco, respectively, interfered with the induction of JA upon herbivore feeding (Halitschke \& Baldwin, 2003; Zhou et al., 2009; Lu et al., 2015). Likewise, in a recent study in barley, overexpression and down-regulation of LOX2.2 affected the expression of JA-related genes depicting its 
role in JA-biosynthesis (Losvik et al., 2017).

The present study used gene silencing of CaLOX2, instead of transformation, and demonstrates that CaLOX2 interferes with the production of not only JA and but also other jasmonates, such as OPDA and JA-lle, upon thrips feeding on pepper, underlining its role in the octadecanoid pathway. Moreover, our data show that targeting a specific 13-type LOX gene in a non-model plant can be effective to suppress the entire jasmonate cascade.

Silencing CaLOX2 clearly resulted in enhanced feeding and preference of thrips for CaLOX2-silenced leaves, compared to control leaves. Furthermore, in Nicotiana attenuata and rice, JA-silenced plants were more vulnerable to herbivorous insects (Kessler et al., 2004; Zhou et al., 2009). Moreover, silencing LOX3 in tobacco influenced herbivore community composition under field conditions (Kessler et al., 2004). Such effects are likely the consequence of altered plant phenotype in terms of secondary metabolites or proteinase inhibitors whose biosynthesis is modulated by JA, differentially affecting different members of the plant-associated insect community.

The possiblity exists that JA also regulates the defense of pepper, indirectly, by influencing herbivore-induced plant volatiles, thereby mediating the attraction of carnivorous arthropods, such as parasitoids or predators of thrips, since the importance of JA in attraction of carnivorous enemies of herbivores has been reported for several plant species (Dicke et al., 1999; Gols et al., 2003; Halitschke et al., 2008; Ozawa et al., 2008). The CaLOX2-silenced plants will be useful to investigate the role of this gene in indirect defense of sweet pepper.

\section{Conclusion}

This study has shown that gene silencing by VIGS is a useful method to functionally characterize candidate genes of pepper for their role in resistance to insects. Through a multidisciplinary approach, involving in-silico, transcriptional, chemical, behavioral studies and bioassays, to assess plant resistance, this study identified CaLOX2 as being involved in the JA-biosynthetic pathway. Moreover, JA-dependent signaling was shown to be important in defense of pepper plants to thrips. Thus, this method allows for investigating functional roles of $C$. annuum genes in plant-insect interactions.

\section{Acknowledgements}

We thank Gerrie Wiegers for help with thrips culture and Wageningen University \& Research experimental farm (Unifarm) for plant propagation. This work is part of the Perspective programme Green Defences Against Pests which is partly financed by 
the Netherlands Organisation for Scientific Research (NWO) and partly by the breeding companies Keygene, Syngenta and EastWest Seeds. KB received funding from an NWO-STW-VENI grant. The authors have no conflict of interest.

\section{References}

Abe H, Ohnishi J, Narusaka M, Seo S, Narusaka Y, Tsuda S, Kobayashi M. 2008. Function of jasmonate in response and tolerance of Arabidopsis to thrip feeding. Plant Cell Physiology 49(1): 68-80.

Abe H, Shimoda T, Ohnishi J, Kugimiya S, Narusaka M, Seo S, Narusaka Y, Tsuda S, Kobayashi M. 2009. Jasmonate-dependent plant defense restricts thrips performance and preference. BMC Plant Biology 9: 97.

Abe H, Tomitaka Y, Shimoda T, Seo S, Sakurai T, Kugimiya S, Tsuda S, Kobayashi M. 2012. Antagonistic plant defense system regulated by phytohormones assists interactions among vector insect, thrips and a tospovirus. Plant and Cell Physiology 53(1): 204-212.

Allmann S, Halitschke R, Schuurink RC, Baldwin IT. 2010. Oxylipin channelling in Nicotiana attenuata: lipoxygenase 2 supplies substrates for green leaf volatile production. Plant Cell and Environment 33(12): 2028-2040.

Ament K, Kant MR, Sabelis MW, Haring MA, Schuurink RC. 2004. Jasmonic acid is a key regulator of spider mite-induced volatile terpenoid and methyl salicylate emission in tomato. Plant Physiology 135(4): 2025-2037.

Bailly C, Bogatek-Leszczynska R, Come D, Corbineau F. 2002. Changes in activities of antioxidant enzymes and lipoxygenase during growth of sunflower seedlings from seeds of different vigour. Seed Science Research 12(1): 47-55.

Barry CS, Giovannoni JJ. 2007. Ethylene and fruit ripening. Journal of Plant Growth Regulation 26(2): 143-159.

Bell E, Creelman RA, Mullet JE. 1995. A chloroplast lipoxygenase is required for wound-induced jasmonic acid accumulation in Arabidopsis. Proceedings of the National Academy of Sciences of the United States of America 92(19): 8675-8679.

Bonaventure G. 2012. Perception of insect feeding by plants. Plant Biology 14(6): 872-880.

Brash AR. 1999. Lipoxygenases: Occurrence, functions, catalysis, and acquisition of substrate. Journal of Biological Chemistry 274(34): 23679-23682.

Chen Z, Chen X, Yan HW, Li WW, Li Y, Cai RH, Xiang Y. 2015. The lipoxygenase gene family in Poplar: Identification, classification, and expression in response to MeJA treatment. PLoS One 10(4): e0125526.

De Vos M, Van Oosten VR, Van Poecke RMP, Van Pelt JA, Pozo MJ, Mueller MJ, Buchala AJ, Metraux JP, Van Loon LC, Dicke M, Pieterse CMJ. 2005. Signal signature and transcriptome changes of Arabidopsis during pathogen and insect attack. Molecular Plant-Microbe Interactions 18(9): 923-937.

Dicke M, Gols R, Ludeking D, Posthumus MA. 1999. Jasmonic acid and herbivory differentially induce carnivore-attracting plant volatiles in lima bean plants. Journal of Chemical Ecology 25(8): 1907-1922.

Escobar-Bravo R, Klinkhamer PGL, Leiss KA. 2017. Induction of jasmonic acid-associated defenses by thrips alters host suitability for conspecifics and correlates with increased trichome densities in Tomato. Plant and Cell Physiology 58(3): 622-634. 
Feussner I, Wasternack C. 2002. The lipoxygenase pathway. Annual Review of Plant Biology 53: 275-297.

Finn RD, Bateman A, Clements J, Coggill P, Eberhardt RY, Eddy SR, Heger A, Hetherington K, Holm L, Mistry J, Sonnhammer ELL, Tate J, Punta M. 2014. Pfam: the protein families database. Nucleic Acids Research 42(D1): D222-D230.

Gols R, Roosjen M, Dijkman H, Dicke M. 2003. Induction of direct and indirect plant responses by jasmonic acid, low spider mite densities, or a combination of jasmonic acid treatment and spider mite infestation. Journal of Chemical Ecology 29(12): 2651-2666.

Grostal P, Dicke M. 1999. Direct and indirect cues of predation risk influence behavior and reproduction of prey: a case for acarine interactions. Behavioral Ecology 10(4): 422-427.

Halitschke R, Baldwin IT. 2003. Antisense LOX expression increases herbivore performance by decreasing defense responses and inhibiting growth-related transcriptional reorganization in Nicotiana attenuata. Plant Journal 36(6): 794-807.

Halitschke R, Stenberg JA, Kessler D, Kessler A, Baldwin IT. 2008. Shared signals - 'alarm calls' from plants increase apparency to herbivores and their enemies in nature. Ecology Letters 11(1): 24-34.

Heidel-Fischer HM, Musser RO, Vogel H 2014. Plant transcriptomic responses to herbivory. Annual Plant Reviews: John Wiley \& Sons, Ltd, 155-196.

Howe GA, Jander G. 2008. Plant immunity to insect herbivores. Annual Review of Plant Biology 59: 41-66.

Howe GA, Lightner J, Browse J, Ryan CA. 1996. An octadecanoid pathway mutant (JL5) of tomato is compromised in signaling for defense against insect attack. Plant Cell 8(11): 2067-2077.

Howe GA, Ryan CA. 1999. Suppressors of systemin signaling identify genes in the tomato wound response pathway. Genetics 153(3): 1411-1421.

Hwang IS, Hwang BK. 2010. The Pepper 9-lipoxygenase gene CaLOX1 functions in defense and cell death responses to microbial pathogens. Plant Physiology 152(2): 948-967.

Kessler A, Halitschke R, Baldwin IT. 2004. Silencing the jasmonate cascade: Induced plant defenses and insect populations. Science 305(5684): 665-668.

Kolomiets MV, Hannapel DJ, Chen H, Tymeson M, Gladon RJ. 2001. Lipoxygenase is involved in the control of potato tuber development. Plant Cell 13(3): 613-626.

Li CY, Williams MM, Loh YT, Lee GI, Howe GA. 2002. Resistance of cultivated tomato to cell content-feeding herbivores is regulated by the octadecanoid-signaling pathway. Plant Physiology 130(1): 494-503.

Liu SQ, Liu XH, Jiang LW. 2011. Genome-wide identification, phylogeny and expression analysis of the lipoxygenase gene family in cucumber. Genetics and Molecular Research 10(4): 2613-2636.

Livak KJ, Schmittgen TD. 2001. Analysis of relative gene expression data using real-time quantitative PCR and the 2(T)(-Delta Delta C) method. Methods 25(4): 402-408.

Losvik A, Beste L, Glinwood R, Ivarson E, Stephens J, Zhu LH, Jonsson L. 2017. Overexpression and down-Regulation of barley lipoxygenase LOX2.2 affects jasmonate-regulated genes and aphid fecundity. International Journal of Molecular Sciences 18(12): 2765; doi:2710.3390/ijms18122765.

Lu J, Robert CAM, Riemann M, Cosme M, Mene-Saffrane L, Massana J, Stout MJ, Lou YG, Gershenzon J, Erb M. 2015. Induced jasmonate signaling leads to contrasting effects on 
root damage and herbivore performance. Plant Physiology 167(3): 1100-1116.

Maris PC, Joosten NN, Peters D, Goldbach RW. 2003. Thrips resistance in pepper and its consequences for the acquisition and inoculation of Tomato spotted wilt virus by the western flower thrips. Phytopathology 93(1): 96-101.

Omer AD, Granett J, Karban R, Villa EM. 2001. Chemically-induced resistance against multiple pests in cotton. International Journal of Pest Management 47(1): 49-54.

Ozawa R, Shiojiri K, Sabelis MW, Takabayashi J. 2008. Maize plants sprayed with either jasmonic acid or its precursor, methyl linolenate, attract armyworm parasitoids, but the composition of attractants differs. Entomologia Experimentalis Et Applicata 129(2): 189-199.

Pieterse CMJ, Van der Does D, Zamioudis C, Leon-Reyes A, Van Wees SCM. 2012. Hormonal modulation of plant immunity. Annual Review of Cell and Developmental Biology 28: 489-521.

Podolyan A, White J, Jordan B, Winefield C. 2010. Identification of the lipoxygenase gene family from Vitis vinifera and biochemical characterisation of two 13-lipoxygenases expressed in grape berries of Sauvignon Blanc. Functional Plant Biology 37(8): 767-784.

Porta H, Rocha-Sosa M. 2002. Plant lipoxygenases. Physiological and molecular features. Plant Physiology 130(1): 15-21.

Qin C, Yu CS, Shen YO, Fang XD, Chen L, Min JM, Cheng JW, Zhao SC, Xu M, Luo Y, Yang YL, Wu ZM, Mao LK, Wu HY, Ling-Hu CY, Zhou HK, Lin HJ, Gonzalez-Morales S, Trejo-Saavedra DL, Tian H, Tang X, Zhao MJ, Huang ZY, Zhou AW, Yao XM, Cui JJ, Li WQ, Chen Z, Feng YQ, Niu YC, Bi SM, Yang XW, Li WP, Cai HM, Luo XR, Montes-Hernandez S, Leyva-Gonzalez MA, Xiong ZQ, He XJ, Bai LJ, Tan S, Tang XQ, Liu D, Liu JW, Zhang SX, Chen MS, Zhang L, Zhang L, Zhang YC, Liao WQ, Zhang Y, Wang M, Lv XD, Wen B, Liu HJ, Luan HM, Zhang YG, Yang S, Wang XD, Xu JH, Li XQ, Li SC, Wang JY, Palloix A, Bosland PW, Li YR, Krogh A, Rivera-Bustamante RF, Herrera-Estrella L, Yin Y, Yu JP, Hu KL, Zhang ZM. 2014. Whole-genome sequencing of cultivated and wild peppers provides insights into Capsicum domestication and specialization. Proceedings of the National Academy of Sciences of the United States of America 111(14): 5135-5140.

Rahimi S, Kim YJ, Sukweenadhi J, Zhang DB, Yang DC. 2017. PgLOX6 encoding a lipoxygenase contributes to jasmonic acid biosynthesis and ginsenoside production in Panax ginseng. Journal of Experimental Botany 68(16): 4725-4725.

Royo J, Vancanneyt G, Perez AG, Sanz C, Stormann K, Rosahl S, SanchezSerrano JJ. 1996. Characterization of three potato lipoxygenases with distinct enzymatic activities and different organ-specific and wound-regulated expression patterns. Journal of Biological Chemistry 271(35): 21012-21019.

Sarde S, Kumar A, Remme R, Dicke M. 2018. Genome-wide identification, classification and expression of lipoxygenase gene family in pepper. Plant Molecular Biology: 10.1007/ s11103-11018-10785-y.

Selig P, Keough S, Nalam VJ, Nachappa P. 2016. Jasmonate-dependent plant defenses mediate soybean thrips and soybean aphid performance on soybean. Arthropod-Plant Interactions 10(4): 273-282.

Senthil-Kumar M, Mysore KS. 2014. Tobacco rattle virus-based virus-induced gene silencing in Nicotiana benthamiana. Nature Protocols 9(7): 1549-1562.

Shipp JL, Hao X, Papadopoulos AP, Binns MR. 1998. Impact of western flower thrips (Thysanoptera : Thripidae) on growth, photosynthesis and productivity of greenhouse sweet pepper. Scientia Horticulturae 72(2): 87-102. 
Stam JM, Kroes A, Li YH, Gols R, van Loon JJA, Poelman EH, Dicke M. 2014. Plant interactions with multiple insect herbivores: From community to genes. Annual Review of Plant Biology 65: 689-713.

Steenbergen M, Abd-el-Haliem A, Bleeker P, Dicke M, Escobar-Bravo R, Cheng G, Haring MA, Kant MR, Kappers I, Klinkhamer PGL, Leiss KA, Legarrea S, Macel M, Mouden S, Pieterse CMJ, Sarde SJ, Schuurink RC, De Vos M, Van Wees SCM, Broekgaarden C. 2018. Thrips advisor: exploiting thrips-induced defences to combat pests on crops. Journal of Experimental Botany 69(8): 1837-1848.

Steiner MY. 1990. Determining population characteristics and sampling procedures for the western flower thrips (Thysanoptera, Thripidae) and the predatory mite amblyseius-cucumeris (Acari, Phytoseiidae) on greenhouse cucumber. Environmental Entomology 19(5): 1605-1613.

Tamura K, Peterson D, Peterson N, Stecher G, Nei M, Kumar S. 2011. MEGA5: Molecular evolutionary genetics analysis using maximum likelihood, evolutionary distance, and maximum parsimony methods. Molecular Biology and Evolution 28(10): 2731-2739.

Thaler JS, Humphrey PT, Whiteman NK. 2012. Evolution of jasmonate and salicylate signal crosstalk. Trends in Plant Science 17(5): 260-270.

Thaler JS, Stout MJ, Karban R, Duffey SS. 2001. Jasmonate-mediated induced plant resistance affects a community of herbivores. Ecological Entomology 26(3): 312-324.

Trapp MA, De Souza GD, Rodrigues E, Boland W, Mithofer A. 2014. Validated method for phytohormone quantification in plants. Frontiers in Plant Science 5: 417.

Tzin V, Fernandez-Pozo N, Richter A, Schmelz EA, Schoettner M, Schafer M, Ahern KR, Meihls LN, Kaur H, Huffaker A, Mori N, Degenhardt J, Mueller LA, Jander G. 2015. Dynamic maize responses to aphid feeding are revealed by a time series of transcriptomic and metabolomic assays. Plant Physiology 169(3): 1727-1743.

Umate P. 2011. Genome-wide analysis of lipoxygenase gene family in Arabidopsis and rice. Plant Signaling \& Behavior 6(3): 335-338.

Vandesompele J, De Preter K, Pattyn F, Poppe B, Van Roy N, De Paepe A, Speleman F. 2002. Accurate normalization of real-time quantitative RT-PCR data by geometric averaging of multiple internal control genes. Genome Biology 3(7): RESEARCH0034.

Walling LL. 2000. The myriad plant responses to herbivores. Journal of Plant Growth Regulation 19(2): 195-216.

Walling LL. 2008. Avoiding effective defenses: Strategies employed by phloem-feeding insects. Plant Physiology 146(3): 859-866.

Wang JE, Li DW, Gong ZH, Zhang YL. 2013. Optimization of virus-induced gene silencing in pepper (Capsicum annuum L.). Genetics and Molecular Research 12(3): 2492-2506.

Wasternack C. 2007. Jasmonates: An update on biosynthesis, signal transduction and action in plant stress response, growth and development. Annals of Botany 100(4): 681-697.

Welter SC, Rosenheim JA, Johnson MW, Mau RFL, Gusukumaminuto LR. 1990. Effects of thrips-palmi and western flower thrips (Thysanoptera, Thripidae) on the yield, growth, and carbon allocation pattern in cucumbers. Journal of Economic Entomology 83(5): 20922101.

Xie DX, Feys BF, James S, Nieto-Rostro M, Turner JG. 1998. COl1: An Arabidopsis gene required for jasmonate-regulated defense and fertility. Science 280(5366): 1091-1094.

Yan LH, Zhai QZ, Wei JN, Li SY, Wang B, Huang TT, Du MM, Sun JQ, Kang L, Li CB, Li CY. 2013. Role of tomato lipoxygenase $D$ in wound-induced jasmonate biosynthesis and plant 
immunity to insect herbivores. PLoS Genetics 9(12): e1003964.

Yates A, Akanni W, Amode MR, Barrell D, Billis K, Carvalho-Silva D, Cummins C, Clapham P, Fitzgerald S, Gil L, Giron CG, Gordon L, Hourlier T, Hunt SE, Janacek SH, Johnson N, Juettemann T, Keenan S, Lavidas I, Martin FJ, Maurel T, McLaren W, Murphy DN, Nag R, Nuhn M, Parker A, Patricio M, Pignatelli M, Rahtz M, Riat HS, Sheppard D, Taylor K, Thormann A, Vullo A, Wilder SP, Zadissa A, Birney E, Harrow J, Muffato M, Perry E, Ruffier M, Spudich G, Trevanion SJ, Cunningham F, Aken BL, Zerbino DR, Flicek P. 2016. Ensembl 2016. Nucleic Acids Research 44(D1): D710-D716.

Zhang B, Chen KS, Bowen J, Allan A, Espley R, Karunairetnam S, Ferguson I. 2006. Differential expression within the LOX gene family in ripening kiwifruit. Journal of Experimental Botany 57(14): 3825-3836.

Zhang C, Jin YZ, Liu JY, Tang YF, Cao SX, Qi HY. 2014. The phylogeny and expression profiles of the lipoxygenase (LOX) family genes in the melon (Cucumis melo L.) genome. Scientia Horticulturae 170: 94-102.

Zheng SJ, van Dijk JP, Bruinsma M, Dicke M. 2007. Sensitivity and speed of induced defense of cabbage (Brassica oleracea $L$.): Dynamics of BoLOX expression patterns during insect and pathogen attack. Molecular Plant-Microbe Interactions 20(11): 1332-1345.

Zheng SJ, Zhang PJ, van Loon JJA, Dicke M. 2011. Silencing defense pathways in arabidopsis by heterologous gene sequences from Brassica oleracea enhances the performance of a specialist and a generalist herbivorous insect. Journal of Chemical Ecology 37(8): 818-829.

Zhou GX, Qi JF, Ren N, Cheng JA, Erb M, Mao BZ, Lou YG. 2009. Silencing OsHI-LOX makes rice more susceptible to chewing herbivores, but enhances resistance to a phloem feeder. Plant Journal 60(4): 638-648.

Zhu-Salzman K, Salzman RA, Ahn JE, Koiwa H. 2004. Transcriptional regulation of sorghum defense determinants against a phloem-feeding aphid. Plant Physiology 134(1): 420-431.

Zust T, Agrawal AA. 2016. Mechanisms and evolution of plant resistance to aphids. Nature Plants 2(1): 15206. 




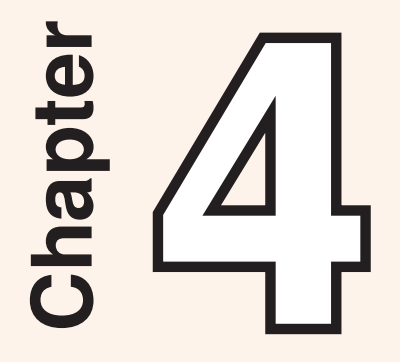

\section{Whole-genome transcriptional reprogramming of sweet pepper in response to Western flower thrips feeding}

SJ. Sarde*, R. Hickman*, M. Steenbergen, G. Wiegers, CMJ. Pieterse, SCM. van Wees and M. Dicke

${ }^{*}$ These authors contributed equally to this work. 


\section{Abstract}

Plants respond to insect herbivory with extensively reprogramming their transcriptome. This dynamic process shapes downstream phenotypic changes. To capture the details of this dynamic transcriptomic response, high-density RNA-Seq analysis of the onset of the response is a powerful tool. We performed a high-density time-series RNA-Seq analysis on leaf tissue of sweet-pepper plants at 12 time points within the first 9 hours of feeding by the cell-content-feeding Western flower thrips (Frankliniella occidentalis). Over 3000 pepper genes ( $8.6 \%$ of the pepper transcriptome) responded to thrips feeding, representing 23 distinct co-expressed gene clusters, 16 up-regulated and 7 down-regulated. Up-regulation occurred fast and was predominantly associated with defence, while down-regulation was slower and associated with developmental processes. The transcription factor families ERF, MYB, NAC, bHLH and WRKY emerged as major regulators of the sweet-pepper response. Chronology analysis showed sequential activation of genes associated with jasmonic acid and ethylene pathways and with the biosynthesis of defence-related phenylpropanoids, flavonoids and terpenoids.Comparative transcriptomic analysis of sweet pepper and Arabidopsis responses to $F$. occidentalis feeding revealed overlapping core and plant species-specific responses. Activation of JA pathway genes is part of the core response, while activation of genes involved in isoprenoid biosynthesis is among the sweet pepper-specific responses.

Keywords: Pepper, thrips, RNA-Seq, transcriptomics, high-resolution time series, defence, phytohormones, secondary metabolites 


\section{Introduction}

Since 400 million years, plants and herbivorous insects interact (Labandeira, 2007). The total number of herbivorous insect species is estimated to be around three million (Schoonhoven et al., 2005). Based on the feeding guild, herbivorous insects can be broadly classified into chewers, phloem feeders and cell-content feeders (Stam et al., 2014). To protect themselves against herbivorous insects, plants have evolved a plethora of direct and indirect defence mechanisms. In direct defence, plant traits influence the performance of herbivorous insects, e.g. by the production of secondary metabolites that influence herbivore growth or mortality (Howe \& Jander, 2008; War et al., 2012). In contrast, indirect defence of plants promotes the effectiveness of the enemies of herbivorous insects. Indirect defence may include the release of a cocktail of volatile compounds, so-called herbivore-induced plant volatiles (HIPVs), that attract predators and parasitoids that attack the herbivores (Dicke, 2009; McCormick et al., 2012; Mithofer \& Boland, 2012; Dicke, 2015).

Plants recognize herbivorous insects through herbivore-associated molecular patterns (HAMPs) or damage-associated molecular patterns (DAMPs) (Mithofer \& Boland, 2008; Bonaventure, 2012; Heidel-Fischer et al., 2014; Duran-Flores \& Heil, 2016). Upon herbivore perception, signal-transduction pathways are activated that underlie the induction of defences. This includes an extensive, dynamic transcriptional reorganisation (Kessler \& Baldwin, 2002; De Vos et al., 2005; Howe \& Jander, 2008; Bidart-Bouzat \& Kliebenstein, 2011; Bonnet et al., 2017; Hickman et al., 2017). This complex transcriptional response includes the up-regulation and down-regulation of large numbers of genes, including genes encoding enzymes of phytohormone biosynthetic and response pathways, biosynthetic pathways of primary and secondary metabolites, developmental processes, as well as genes encoding transcription factors. The transcriptional response consists of a plethora of responses with different temporal patterns (Breeze et al., 2011; Windram et al., 2012; Hickman et al., 2017). This complex of temporal transcriptional responses orchestrates the activation and attenuation of various processes, influencing the plant's phenotype. Important progress has been made in analysing the temporally dynamic transcriptomic responses of Arabidopsis thaliana to insect herbivory (De Vos et al., 2005; Bidart-Bouzat \& Kliebenstein, 2011; Coolen et al., 2016; Davila Olivas et al., 2016) but only limited information is available for other plant species (Bonnet et al., 2017; Durrant et al., 2017). Most of these studies address chewing and phloem-sucking herbivores, whereas information on cell-content feeders is limited.

An early component of phenotypic reprogramming in response to attack involves the accumulation of and changed sensitivity to phytohormones, such as jasmonic acid 
$(\mathrm{JA})$, salicylic acid $(\mathrm{SA})$, and ethylene $(\mathrm{ET})$ that regulate plant defence responses (Maffei et al., 2007; Verhage et al., 2010; Erb et al., 2012; Pieterse et al., 2012; Stam et al., 2014). For instance, SA is especially induced by phloem-feeding insects like aphids (Zhu-Salzman et al., 2004; Walling, 2008; Pieterse et al., 2012; Tzin et al., 2015), whereas JA is induced by chewers and cell-content feeders like caterpillars and thrips, respectively (De Vos et al., 2005; Abe et al., 2008; Abe et al., 2009; Pieterse et al., 2012). Moreover, other major phytohormones like abscisic acid, cytokinins, auxin and gibberellins are also reported to act in herbivore-induced defence mechanisms (Pieterse et al., 2012; Stam et al., 2014). JA is a prominent phytohormone modulating induced plant defences against thrips feeding (De Vos et al., 2005; Abe et al., 2008). A microarray-based whole-genome transcriptome study of Arabidopsis (3 time points over $72 \mathrm{~h}$ ) showed that $69 \%$ of the thrips-responsive genes were JA responsive (De Vos et al., 2005). In tomato, reduced resistance was observed in the JA-defective mutant Defenceless1 (Def1) and in and Arabidopsis in the JA-insensitive coi1-1 mutant (Li et al., 2002; Escobar-Bravo et al., 2017). In Arabidopsis and Chinese cabbage (Brassica rapa subsp. pekinensis), feeding by the cell-content feeding generalist insect herbivore Western flower thrips (WFT; Frankliniella occidentalis) (Pergande) (Thysanoptera: Thripidae) induced the expression of JA-related marker genes, resulting in elevated levels of JA (Abe et al., 2008; Abe et al., 2009). Moreover, exogenous application of JA on several crops resulted in elevated resistance to thrips feeding (Omer et al., 2001; Thaler et al., 2001; Selig et al., 2016).

Evidence from several whole-genome transcriptome studies shows that plants differentially respond to different environmental stresses with a high degree of specificity. Several studies have shown how Arabidopsis specifically rearranges its transcriptome over time against different biotic and abiotic stresses (De Vos et al., 2005; Breeze et al., 2011; Windram et al., 2012; Coolen et al., 2016; Davila Olivas et al., 2016; Hickman et al., 2017). In plant-herbivore interactions, the majority of transcriptomic reconfigurations occurs early (minutes to hours) during the interaction, shaping the subsequent plant response to herbivory (Maffei et al., 2007; Stam et al., 2014).

WFT is a devastating pest insect hampering pepper yield worldwide. Thrips are minute cell-content feeding insects exerting direct damage to plants by piercing into epidermal cells and ingesting the contents of mesophyll cells. Their feeding damage results in reflective 'silver scars' on plant tissues hampering plant photosynthetic capacity, growth, reproduction and eventually yield (Steiner, 1990; Welter et al., 1990; Shipp et al., 1998; Steenbergen et al., 2018). This herbivore is challenging to control with pesticides due to its thigmokinetic life-style and extensive resistance to pesticides. Additionally, WFT causes indirect damage by acting as vector of tospoviruses, such as Tomato spotted wilt virus (TSWV) (Maris et al., 2003). Therefore, to develop thrips-resistant crop varieties to minimize the damage, exploration and understanding 
of the genetic basis underlying plant defence responses is vital (Steenbergen et al., 2018). Such crop varieties may be a valuable component of integrated pest management, in combination with biological control (Mouden et al., 2017).

Here, we present an in-depth analysis of the dynamic transcriptomic response of sweet-pepper plants to feeding by WFT through a high-resolution RNA-Seq analysis of the early temporal response. We implemented a state-of-the-art bioinformatics approach to gain in-depth insights into the transcriptomic response of sweet pepper. The key objectives of this study were: (1) To assess the temporal transcriptional reprogramming of sweet pepper in response to WFT feeding, (2) to identify co-expressed gene clusters and their involvement in biological pathways, (3) to identify the major transcription factor (TF) families involved and their binding motifs to unravel directional regulatory connections with downstream regulated genes and their involvement in biological processes, (4) to investigate the chronology of phytohormonal and secondary metabolite pathways underlying induced plant defence, (5) to unravel the conservation of induced responses to WFT feeding between Arabidopsis and sweet pepper.

\section{Materials and methods}

\section{Plants and thrips}

Sweet pepper, Capsicum annuum (Mandy variety, Rijk Zwaan (De Lier, The Netherlands)), seeds were sown in $12 \mathrm{~cm}$ pots in a greenhouse at $23-25^{\circ} \mathrm{C}, 16 \mathrm{~L}: 8 \mathrm{D}$ photoperiod and $70 \pm 10 \%$ relative humidity. Two weeks later, plants were individually transplanted into $14 \mathrm{~cm}$ diameter pots and transferred to a greenhouse with controlled conditions (16L:8D photoperiod, $60 \pm 10 \%$ relative humidity, $23 \pm 5^{\circ} \mathrm{C}$ diurnal and $20 \pm 5^{\circ} \mathrm{C}$ nocturnal temperatures). Four-week-old sweet pepper plants were used for experiments. Adult females of Western flower thrips (Frankliniella occidentalis) reared on chrysanthemum plants were collected and transferred to bean pods (Phaseolus vulgaris) in glass jars (10 cm diameter). The jars were incubated in a climate-controlled cabinet with $16 \mathrm{~L}: 8 \mathrm{D}$ photoperiod, $25 \pm 2^{\circ} \mathrm{C}$ and $70 \pm 10 \%$ relative humidity to produce larvae for the experiments (Sarde et al., 2018a; Sarde et al., 2018b).

\section{RNA-Seq experimental setup}

\section{Treatment and sampling}

Four-week-old sweet pepper plants (having four fully expanded leaves) were each infested with five second instar (L2) WFT larvae, confined in a clip cage $(3 \mathrm{~cm}$ diameter) on one of the first two leaves. Empty clip cages were used as mock treatment. For each time point and treatment the leaf area in the clip cage (for both, mock and 
thrips treatment) was harvested using a cork borer ( $3 \mathrm{~cm}$ diameter), snap frozen in liquid nitrogen and stored at $-80^{\circ} \mathrm{C}$; three biological replicates were collected, each biological replicate consisting of one individual plant. Time points of sampling were 0 , $0.5,1.0,1.5,2.0,2.5,3.0,4.0,5.0,6.0,7.0,8.0,9.0 \mathrm{~h}$ of infestation.

\section{$\underline{R N A \text { extraction and library preparation }}$}

RNA extraction was performed using the Qiagen RNeasy Plant Mini Kit including DNase I treatment, following the company's instructions. RNA quantity was assessed using Nanodrop. RNA quality was assessed using RNA Integrity Number (RIN) with Agilent 2100 bioanalyzer. For RNA library preparation, samples with RIN values $\geq 7$ were used.

For preparation of the RNA-Seq library and subsequent sequencing, samples were processed according to the TruSeq Stranded mRNA HT Sample Prep Kit from Illumina (Illumina Inc., San Diego, CA, USA). This protocol allows the identification of strand-specific transcripts. Samples were sequenced with an Illumina Hi-seq 2000 platform. Samples were randomly assigned to seven lanes of the Illumina flow cells within each run.

\section{Analysis of RNA-Seq dataset}

\section{Processing of raw RNA-Seq data}

The RNA-Seq raw reads were subjected to quality control with the FastQC (https:// www.bioinformatics.babraham.ac.uk/projects/fastqc/) tool. Trimmomatic was used to trim the initial 14-basepairs (bp) and Illumina adapters. Reads below 25 bp length were excluded from analysis for all samples (Bolger et al., 2014).

\section{Alignment and quantification of RNA-Seq data}

Trimmed RNA-Seq reads were aligned to the Capsicum annuum L. Zunla genome using TopHat (v2.0.14) (Kim et al., 2013) with the following parameters: 'p 4', '--minintron-length 40', '--max-intron-length 2000', '--bowtie-n', '-N 4', '--read-gap-length 2', '--read-edit-dist 4', '--no-novel-juncs'. The aligned reads to each $C$. annuum Zunla gene model were summarized using HTSeq-count (v.0.9.1) (Anders et al., 2015) with parameters: '--stranded no', '-i ID', '-t mRNA'. Principal component analysis (PCA) was performed using the DESeq2 package on regularized $\log _{2}$-transformed data (Love et al., 2014; Love et al., 2015) in R (https://www.r-project.org/).

\section{Differential gene expression analysis}

Differential gene expression (DEG) analysis was executed with the DESeq2 Bioconductor package (Love et al., 2014; Love et al., 2015) in R. Prior to analysis, raw read counts were normalized for sequencing depth across all samples using DESeq2's 
median-count normalization procedure. To identify genes that were differentially expressed between mock and thrips-treated plants we used a negative binomial likelihood ratio test (nbinomLRT) considering treatment and time post treatment as factors. Genes with Bonferroni corrected $P$ value $<0.01$, $\log _{2}$-fold change $\geq 0.5$ or $\leq-0.5$ at one or more time points and read counts $\geq 20$ at least in one sample were considered differentially expressed.

\section{Clustering of differentially expressed genes}

Temporal expression profiles of the DEGs were clustered using SplineCluster (Heard et al., 2006). For this, $\log _{2}$-fold change profiles of DEGs at each time point (thrips-treated versus mock), were used with a prior precision stringency of $10^{-4}$, cluster reallocation step and the default normalization procedure (Heard, 2011). Default settings were maintained for all other optional parameters.

\section{TF family and promoter motif enrichment analyses}

To determine which TF families were enriched among the genes differentially expressed in response to thrips feeding, we tested for overrepresentation of genes encoding members of different TF families found in sweet pepper. Previously, the $C$. annuum Zunla TF families were determined using C. annuum cv. CM334 TFs (1665 TFs) from the Plant Transcription Factor Database (http://planttfdb.cbi.pku.edu.cn/ index.php) using blastp with stringency E-value $<10^{-4}$. Overrepresentation of TF families within a set of genes was analyzed using the hypergeometric distribution. $P$ values were corrected for multiple testing with the Bonferroni method.

For the promoter motif analysis, characterized Arabidopsis TF DNA-binding motifs were retrieved from CIS-DB version 1.02 (Weirauch et al., 2014) and those described in Franco-Zorrilla et al. (2014). Promoter sequences defined as the 500 bp upstream of the translation start site were retrieved for all $C$. annuum Zunla genes. The occurrence of a motif within a promoter was determined using FIMO (Grant et al., 2011), where a promoter was considered to contain a motif if it had at least one match with a $P$ value $<10^{-4}$. Motif enrichment was assessed using the hypergeometric distribution against the background of all sweet pepper genes.

\section{Identification of chronology of defence pathways upon thrips feeding}

To identify the chronology of defence pathways in sweet pepper leaves upon thrips feeding, we performed a pairwise comparison between mock and thrips-treated samples at each time point to determine the time point at which DEGs were first differentially expressed (first time of differential expression, $P<0.01$, $\log _{2}$-fold change $>0.5$ (up-regulated) and <-0.5 (down-regulated) using DESeq2 (Love et al., 2014; Love et al., 2015). For the small number of genes that did not meet these criteria, the time point of first differential expression was defined by minimal $P$ value. 


\section{Gene Ontology (GO) annotation of the C. annuum Zunla variety}

For $\mathrm{GO}$ annotation of the $C$. annuum Zunla proteome, we implemented a comparative genomics approach on the $C$. annuum Zunla proteome (Qin et al., 2014) using the GO-annotated C. annuum cv. CM334 proteome (Plaza database, https://bioinformatics.psb.ugent.be/plaza/) as query (Kim et al., 2014).

\section{Gene Ontology analysis}

For Arabidopsis and sweet pepper, GO enrichment analysis was performed using Cytoscape (Shannon et al., 2003) and GOAtools (v0.7.9) (python-based library), using Fisher's exact test (Klopfenstein et al., 2018), respectively. Overrepresentation of the GO categories "biological process", "cellular component" and "molecular function" were determined at $P<0.05$.

\section{Comparative transcriptomics}

The Bi-directional Best $\underline{H i t}(\mathrm{BBH})$ approach was used on TAIR10 Arabidopsis and $\mathrm{C}$. annuum Zunla proteome using reciprocal blastp with stringency E-value $<10^{-4}$ to determine one-to-one orthologues (Martel et al., 2015). The one-to-one orthologue files were processed and used to investigate commonalities and differences between the Arabidopsis (Steenbergen et al., in prep.) and $C$. annuum transcriptomes as affected by WTF feeding.

\section{Results}

\section{High-resolution transcriptome dynamics in sweet pepper induced by WFT}

To comprehensively and accurately capture the dynamics of the full-genome transcriptional response triggered by thrips feeding in sweet pepper plants, RNA-Seq analysis was carried out for thrips-infested and non-infested plants. Initially, samples from 13 time points were sequenced: three replicates of an initial $0 \mathrm{~h}$ time point plus one replicate for the other 12 time points $(0.5,1.0,1.5,2.0,2.5,3.0,4.0,5.0$, 6.0, 7.0, 8.0, $9.0 \mathrm{~h}$ of infestation) after thrips infestation. The dataset generated was subjected to sample-to-sample distance analysis and PCA, to capture the transcriptome-level relatedness and variation, respectively, within the samples. The data show an increasing difference between the samples with increasing time after the start of thrips infestation, with exception of the 3h time point (PCA, Fig. S1A). Likewise, the sample-to-sample distance heat map (Fig. S1B) clearly shows the close relatedness between sequential time points. Both analyses complement each other and show that the transcriptional response gradually developed over time. Based on this analysis, the 1, 2, 3, 4, 6 and $8 \mathrm{~h}$ time points were selected because they captured the full dynamic range of the transcriptional response to thrips feeding. 
To understand the effect of the factors treatment and time on the transcriptomic profile of sweet pepper plants, all three replicates of the selected six time points were subjected to PCA (Fig. 1A). Both treatment and time explicitly separated the transcriptomes. The difference between mock-treated and thrips-infested plants is small at $1 \mathrm{~h}$ since the initiation of thrips infestation and the difference gradually increases with time. At all six time points the transcriptome of thrips-infested plants is clearly different from that of mock-treated plants. The mock-treated samples exhibit a change in transcriptome with time, suggestive of an effect of circadian rhythm on the transcriptome of sweet pepper plants. Variation within the replicates of thrips-treated samples is higher than for mock-treated samples, which may represent differences in the intensity of thrips feeding among replicates.

Genes that are differentially expressed in thrips-infested and uninfested plants at different time points were identified with a generalized linear model (GLM) using the DESeq2 (Love et al., 2014). This analysis identified a total of 3062 differentially expressed genes (DEGs) (Supplemental Data Set 1), that represent $8.6 \%$ of the total set of pepper transcripts $(35,336)$ (Qin et al., 2014). The JA-biosynthesis genes CaLOX2 and CaAOS, and the JA-regulated genes CaMYC2, CaPIN II are among the DEGs. These representative genes of the JA response show a clear up-regulation within 1-2 h (Fig. 1B), thus supporting the JA-component of the response of sweet pepper to infestation by WFT (Sarde et al., 2018a).

\section{Cluster analysis of DEGs based on expression patterns}

To identify and classify clusters of co-expressed genes responding to thrips feeding over time, the time-series clustering algorithm SplineCluster was employed. A total of 23 clusters, 16 clusters of up-regulated genes and 7 clusters of down-regulated genes (Supplemental Data Set 2) was identified. 2060 DEGs (67.3\%) are represented in the sixteen up-regulated clusters (Clusters 1-16) and 1002 DEGs (32.7\%) in the seven down-regulated clusters (Clusters 17-23) (Fig. 2). This analysis shows that the pepper transcriptional response to thrips feeding is highly dynamic over time. Many changes are initiated between $1 \mathrm{~h}$ and $2 \mathrm{~h}$ after the onset of thrips feeding. The clusters of up-regulated genes show more variation in temporal patterns than the clusters of down-regulated genes (Fig. 2). 
A

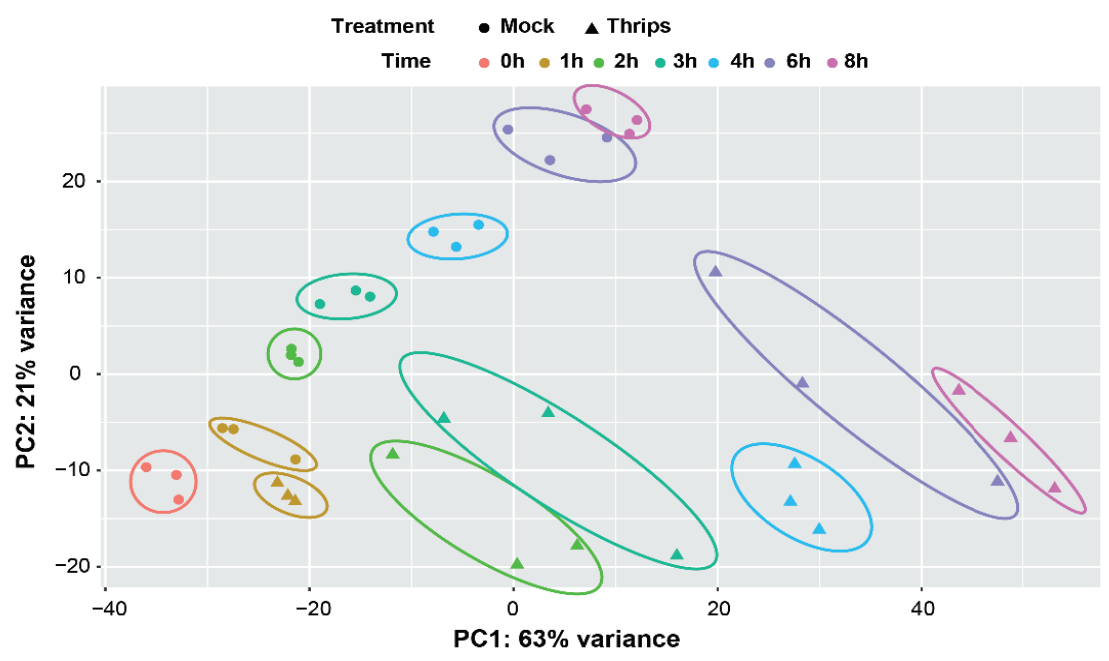

B
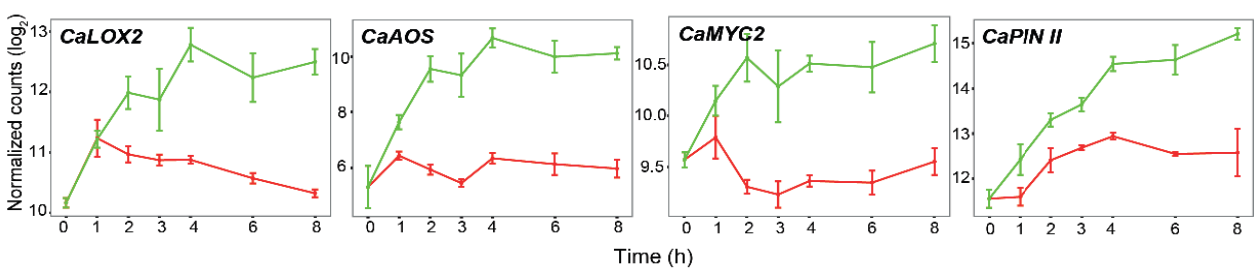

Figure 1. Principal component analysis (PCA) of whole-genome transcriptomic response at different time points for thrips-infested and mock-infested plants plus expression profile of four selected JA-related marker genes of sweet pepper. (A) PCA of sweet pepper transcriptome of non-infested (mock) and infested (thrips) plants; samples were harvested at six time points. PCA was generated on the regularized log2-transformed data within the DESeq2 $\mathrm{R}$ package. Colours and symbol shapes indicate time points and treatments, respectively. Variation explained by the two principal components is depicted on both axes. (B) Expression pattern of selected JA-related marker genes from RNA-Seq dataset. Data represent mean $\pm S E$ ( $n=3$ biological replicates). Red and green colours indicate mock and thrips treatment, respectively.

To investigate biological significance of the co-expressed genes per cluster, we explored overrepresentation of Gene Ontology (GO) terms for the genes per cluster with the python-based library GOAtools (v0.7.9) (Klopfenstein et al., 2018). Each cluster is overrepresented for several functional terms, including unique and common categories (Fig. 2B) (Supplemental Data Sets 3 and 4). As predicted, defence-related and JA-related functional categories are overrepresented in several up-regulated gene clusters. For instance, "response to wounding" is overrepresented in clusters 6 and 11; "response to JA" is overrepresented in clusters 4 and 7 , and "defence response to insects" in cluster 11 . In cluster 5 , "response to salicylic acid" is overrepresented, suggesting that besides JA-related processes also SA-related processes play 
a role in the response of pepper to thrips feeding. Moreover, several up-regulated clusters also exhibited overrepresentation of more unique functional categories. For instance, cluster 1 was specifically enriched for the GO term "tryptophan metabolic process", which is associated with the production of defensive secondary metabolites (Kang \& Back, 2006; Hiruma et al., 2013)and cluster 2 for "terpene/sesquiterpene biosynthetic process", compounds involved in direct and indirect defence against insects (Gershenzon \& Dudareva, 2007) (Supplemental Data Set 3). Down-regulated clusters were associated with GO annotations like "response to auxin", "response to high light", suggesting that plant processes related to growth and development are down-regulated in response to thrips feeding (Supplemental Data Set 4). Taken together, this analysis shows that up-regulated clusters are enriched with genes annotated for being involved in defence-related responses, whereas down-regulated clusters are enriched with genes annotated for being involved in developmental processes. Some clusters harbour genes that are specifically enriched in a biological process that is not represented by other clusters, while especially JA-associated processes are commonly enriched by several clusters.

\section{TF family abundance and TF binding motif analysis}

TFs are important regulators of transcriptional responses and, thus, of the resulting phenotypic change. They bind to DNA-regulatory sequences in the promoter regions of target genes contributing to modulation of gene expression. The investigation of $C$. annuum Zunla TF families using 1665 TFs of $C$. annuum $\mathrm{cv}$. CM334 identified a total 1424 (unique or non-redundant) TFs in C. annuum Zunla (Supplemental Data Set 5). To gain insight in regulators of the pepper transcriptome in response to thrips infestation, we analysed TF family abundance in all up- (2060) and down-regulated (1002) genes. Several TFs belonging to families like ERF, MYB, NAC, bHLH and WRKY are significantly overrepresented among the up-regulated genes (Fig. $3 \mathrm{~A}$ ), suggesting a pivotal role of these TF families in regulating plant responses in sweet pepper plants that are induced upon thrips feeding. Among the down-regulated genes, the TF families HD-ZIP and bHLH are overrepresented. 
A

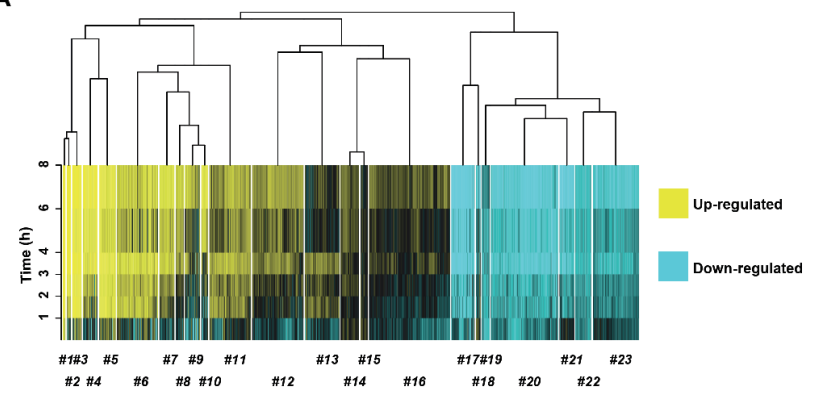

B
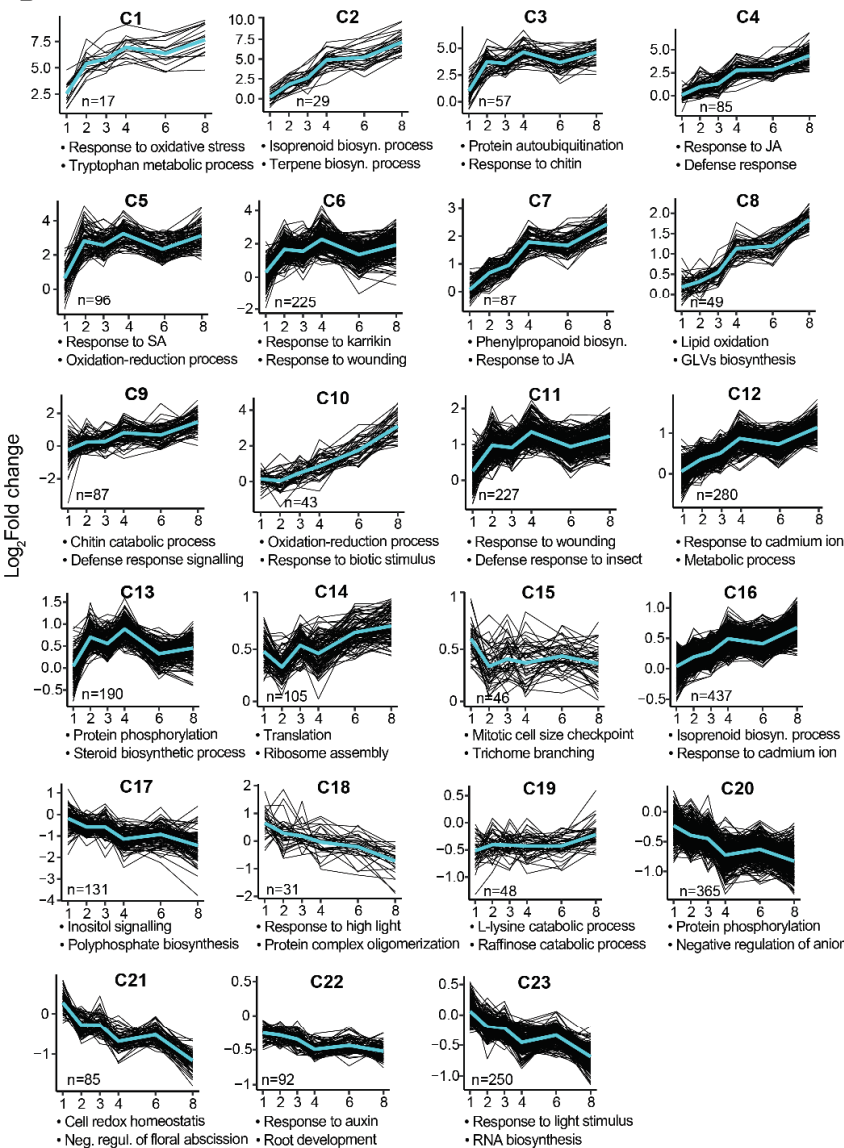

Figure 2. Co-expressed gene clusters of differentially expressed genes in response to feeding by Frankliniella occidentalis. SplineCluster analysis was performed using $\log _{2}$-transformed expression ratios (thrips / mock). (A) Heat map depicting expression profile of 3062 DEGs over time in each cluster. Cluster numbers are depicted with '\#' below the heat map. Yellow and blue indicate up- and down-regulated genes, respectively. (B) Twenty-three partitioned gene clusters (1-16 up- and 17-23 down-regulated) with selected enriched GOterms. The mean expression profile of each cluster is represented by the blue line. The x-axis represents expression pattern of each gene cluster over time (in hours) and y-axis represents $\log _{2}$ fold change of genes from each cluster. 
Because TF binding specificities are typically conserved between related organisms (Portales-Casamar et al., 2010), we extended our analysis of transcriptional regulation underlying the pepper response to thrips by utilizing recently identified DNA binding motifs for 580 Arabidopsis TFs (Franco-Zorrilla et al., 2014; Weirauch et al., 2014), and scanned the promoter sequences of the genes in all 23 co-expressed clusters for overrepresentation of TF binding motifs. In clusters representing up-regulated genes, promoter regions were especially enriched with binding sites for bHLH, ERF, bZIP and WRKY TFs (Fig. 3B). For example, bHLH motifs were particularly enriched in clusters 4 and 7, ERF motifs in clusters 14 and 16, bZIP motifs in clusters 5 and 11 and WRKY motifs in clusters 6 and 16. Motifs that correspond to bHLH and WRKY TF binding sites were overrepresented in clusters involved in JA-related processes (clusters 4 and 7; Supplemental Data Set 3) and SA-related responses (cluster 6; Supplemental Data Set 3), respectively. In down-regulated clusters, Dof, MIKC, MYB-related and TCP TF-specific binding sites are overrepresented (Fig. 3B). In contrast to TF abundance analysis (Fig. 3A), no enrichment of bHLH binding motifs was found in genes of down-regulated clusters (Fig. 3B). Collectively, the expression profiles of pepper TFs and the enrichment of their binding sites in the thrips-induced pepper transcriptome provides important insight into the architecture of the gene regulatory network of pepper in response to thrips infestation.

\section{Chronology of sweet pepper defence response upon thrips feeding}

The transcriptional response to thrips feeding develops with time (Fig. 1). To understand the chronology of biological processes activated upon thrips feeding, we performed a pairwise comparison (mock versus thrips-treated) for each time point on the temporal RNA-Seq dataset (Fig. 4). This analysis highlights the dynamic transcriptome profile and distinguishes DEGs (up- and down-regulated) that are differentially expressed for the first time (irst time of differential expression (ftode)) and DEGs that become again differentially expressed (ade) (Fig. 4) (Supplemental Data Set 6). At $2 \mathrm{~h}$ after the introduction of thrips ca 10 times more genes are up-regulated than down-regulated. The majority of DEGs (up- and down-regulated) became first differentially expressed within $4 \mathrm{~h}$ and $8 \mathrm{~h}$ since the onset of thrips feeding with twice as many ftode genes being up-regulated compared to ftode genes being down-regulated. 
A

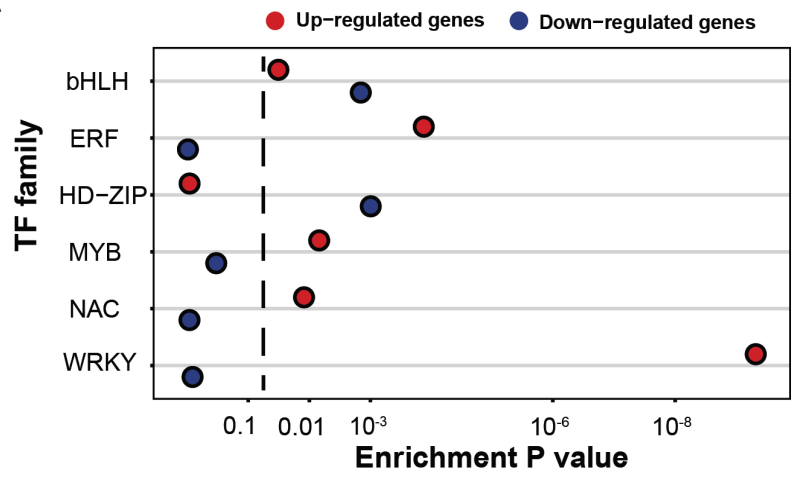

B

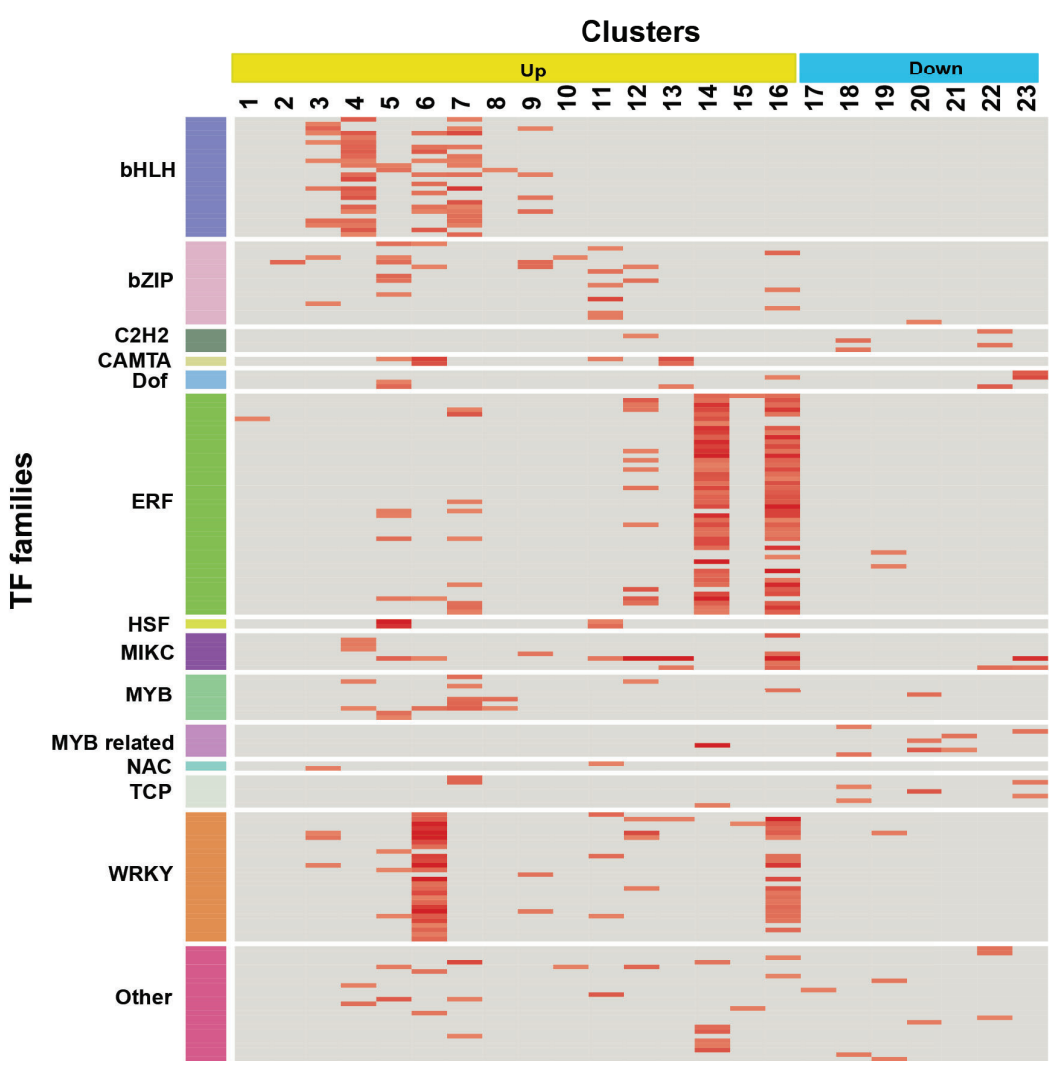

Figure 3. Overrepresented TF families and enriched TF DNA binding motifs in differentially expressed gene clusters. (A) TF families among up-regulated (red) and down-regulated (blue) genes upon WFT feeding. Black dotted line represents significance threshold $(P<$ 0.05). (B) Enriched TF DNA binding motifs in promotors of genes in up- and down-regulated gene clusters. The red colour intensity corresponds to raw $P$ value of enriched motifs. Columns and rows represent cluster numbers and enriched motifs, respectively. Clusters 1-16 are up-regulated and clusters 17-23 are down-regulated. 


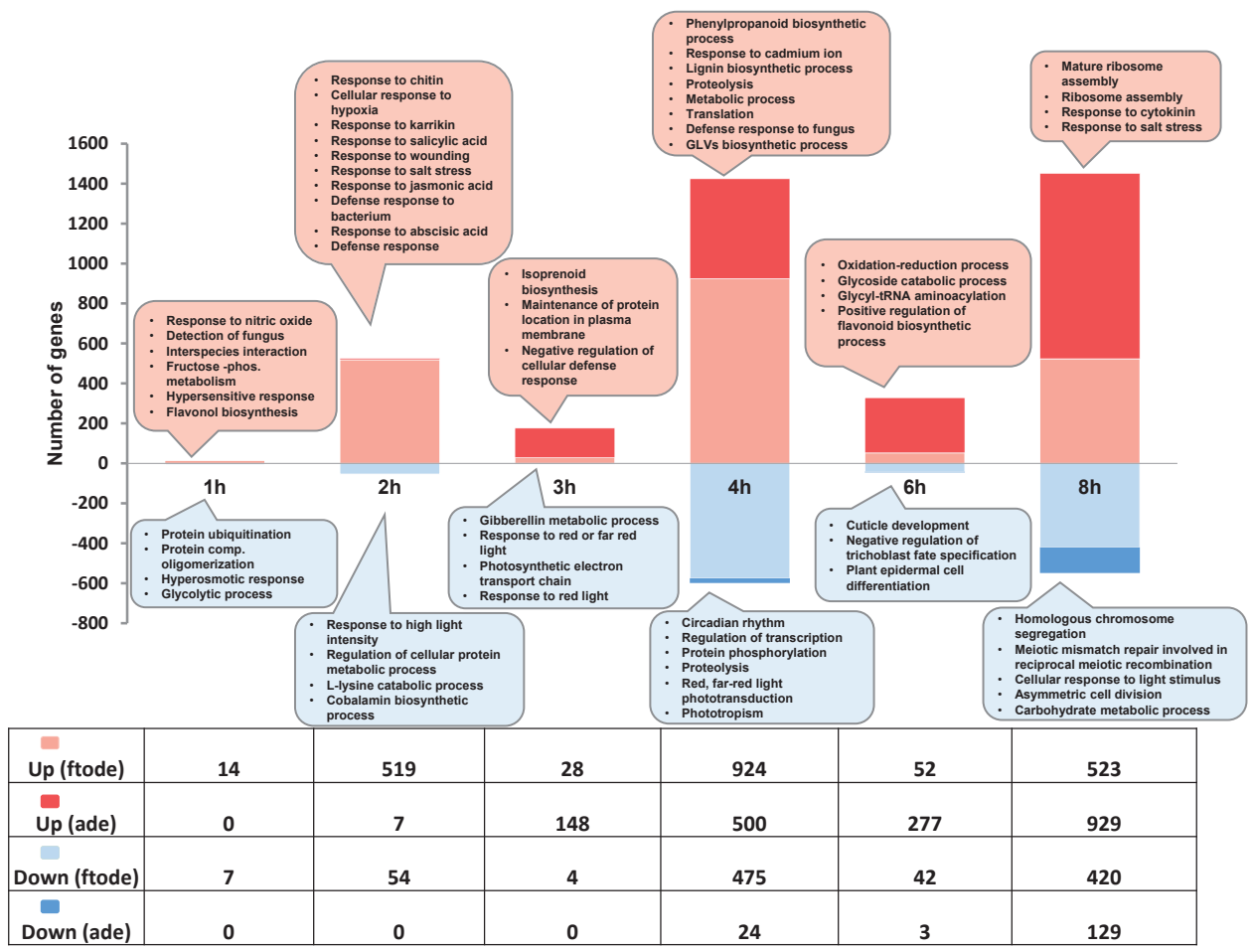

Figure 4. Chronology of sweet pepper transcriptome profile and defence pathways on Western flower thrips feeding. Number of genes responding at each time point are depicted in the legend under the figure. Selected GO terms for the first time of differential expression (ftode) genes are shown for each time point. Light and dark blue colours depict up-regulated first time of differentially expressed (ftode) genes and again differentially expressed (ade) genes, respectively. Light and dark orange depicts down-regulated first time of differentially expressed (ftode) genes and again differentially expressed (ade) genes.

Among the up-regulated genes at the $2 \mathrm{~h}$ time point, GO terms are mainly associated with hormonal and defence responses, reflecting the induction phase of the defence response to thrips infestation. Likewise, at the $4 \mathrm{~h}$ time point, genes associated with GO terms like "phenylpropanoid biosynthetic process", "lignin biosynthetic process", "green leaf volatile biosynthetic process" are induced, reflecting the onset of direct (phenylpropanoids, lignin) and indirect (green leaf volatiles, terpenoids) defence activation. Among the down-regulated genes, GO terms are associated with growth and development ("gibberellin metabolic processes", "response to red or far red light", "photosynthetic electron transport chain"). Overall, this analysis indicates that the up-regulation of genes in response to thrips infestation occurs faster and more intensively than the down-regulation of genes. Apparently activation of defence-related processes is prioritized over attenuation of growth- and development-related processes. 
A

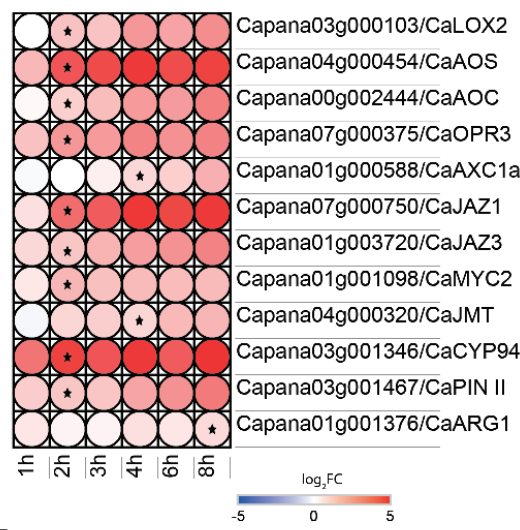

B

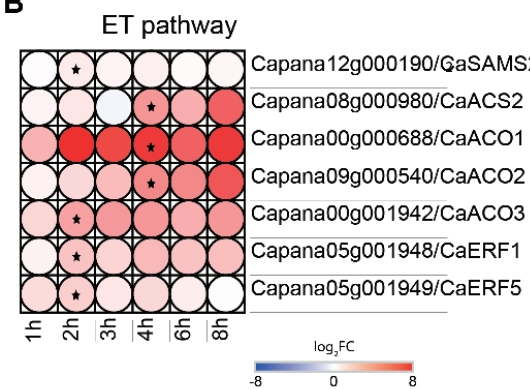

C

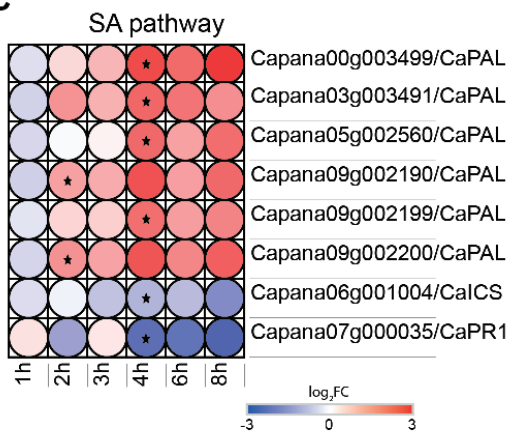

D

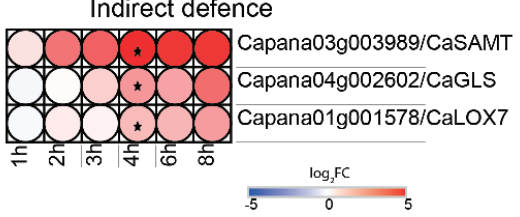

E

Phenylpropanoids and flavonoids

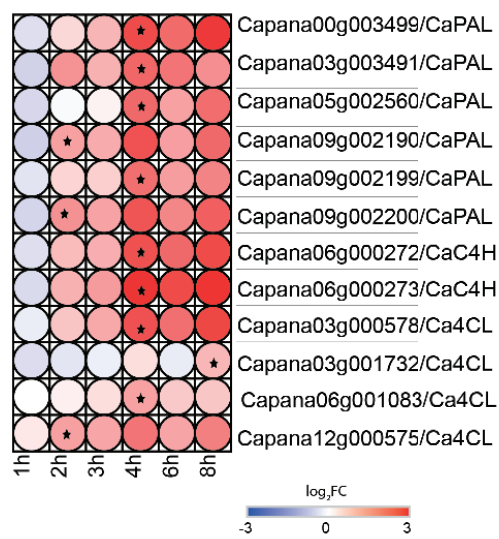

F Isoprenoids, mevalonate (MVA) pathway

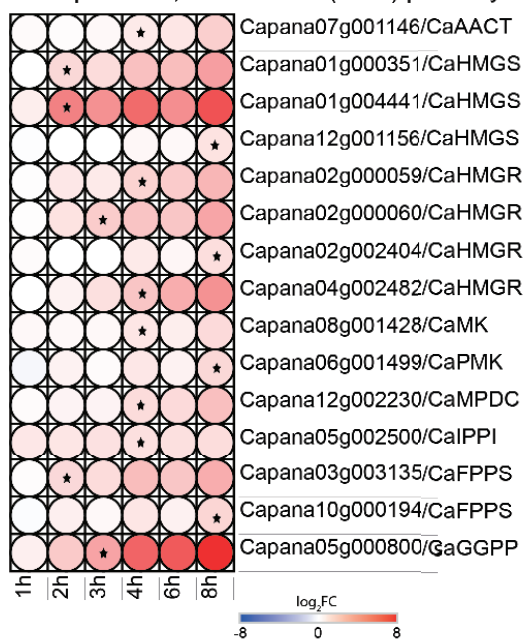

Isoprenoids, non-mevalonate (MEP) pathway

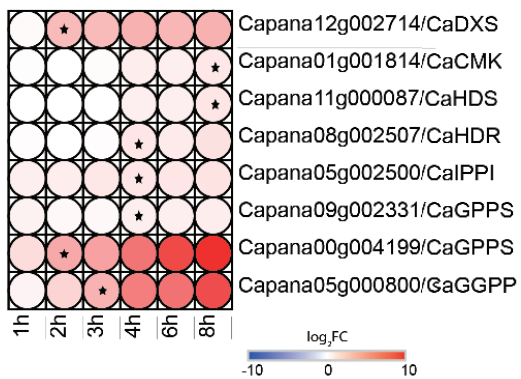

Figure 5. Expression of genes involved in defence-related hormonal cascades, indirect defences and biosynthesis of secondary metabolites. (A) JA pathway, (B) ET pathway, (C) SA pathway, (D) Indirect defence, (E) phenylpropanoid and flavonoid pathways and (F) Isoprenoid pathways (MVA and MEP pathways). $\star$ indicates significant $(P<0.01)$ first time of differential expression (ftode) of each gene. 


\section{Transcription dynamics of genes involved in hormonal and secondary metabo- lite pathways}

To dissect the temporal response of several hormonal and metabolic pathways to thrips feeding, we focused on the expression of genes involved in these pathways. The majority of genes in the JA biosynthetic pathway (CaLOX2, CaAOS, CaAOC, CaOPR3, CaJAZ1, CaJAZ3, CaMYC2, CaCYP94, CaPIN II, CaARG1) are significantly induced at $2 \mathrm{~h}$ after the introduction of thrips onto the plant and sustain up-regulated until the last time point assessed, i.e. 8h after introduction of thrips (Fig. 5A). Likewise, several genes associated with the ET pathway (CaSAMS2, CaACO3, CaERF1, CaERF5) are up-regulated at the $2 \mathrm{~h}$ time point (Fig. 5B). SA biosynthesis in plants can occur via two pathways: the isochorismate synthase (ICS) and phenyl alanine lyase (PAL) pathways; depending on the plant species the induced accumulation of SA can occur primarily through either pathway (Chen et al., 2009). Several homologues of CaPAL are up-regulated at the $4 \mathrm{~h}$ time point, while CalCS and the SA-responsive gene CaPR1 are down-regulated at this time point (Fig. 5C). Furthermore, the genes and their homologues involved in phenylpropanoid and flavonoid biosynthesis ( $\mathrm{Ca} 4 \mathrm{CL}, \mathrm{CaC} 4 \mathrm{H}$ ), including CaPAL homologs, are up-regulated at $4 \mathrm{~h}$ after thrips introduction (Fig. 5E). For the biosynthesis of terpenoids (isoprenoids), an important class of VOCs involved in indirect plant defense in many plant species including pepper (Dicke et al., 1990; Van Den Boom et al., 2004), several genes involved in the MVA (mevalonate) and MEP (methylerythritol 4-phosphate) pathways are significantly induced within 3-4h after introduction of thrips (Fig. 5F). Similarly, other genes involved in the production of VOCs, for example, geranyllinalool synthase (CaGLS) involved in biosynthesis of terpenes, methyl salicylate (MeSA) (CaSAMT) and green leaf volatiles (GLVs) (CaLOX7) were induced after $4 \mathrm{~h}$ of thrips feeding (Fig. 5D). Taken together, the JA and ET pathways were rapidly up-regulated (within $2 \mathrm{~h}$ ), the SA pathway seems down-regulated, whereas genes involved in secondary metabolism and indirect defence are induced at $4 \mathrm{~h}$ after the onset of thrips feeding.

\section{Conservation of defence responses between Arabidopsis and sweet pepper upon WFT feeding}

A similar RNA-Seq analysis of the dynamic response to feeding by $F$. occidentalis has been performed for Arabidopsis, identifying in total 2788 DEGs (1820 up- and 968 down-regulated) for eleven time points distributed over $8 \mathrm{~h}$ of thrips feeding (Steenbergen et al., in prep). In the present study we have identified 3062 DEGs (2060 up- and 1002 down-regulated) for six time points distributed over $8 \mathrm{~h}$ of thrips feeding on pepper. To gain insight in commonalities and differences in the transcriptomic responses of sweet pepper and Arabidopsis, we compared the DEGs recorded in these two studies. In pepper, 981 of the 2060 up-regulated genes and 469 of the 1002 
down-regulated genes, respectively, have Arabidopsis orthologues (Supplementary Data Set 7). In Arabidopsis, 652 of the 1820 up-regulated genes and 392 of the 968 down-regulated genes, respectively, have pepper orthologues. Thus, there is ca 35$50 \%$ overlap in transcriptomic responses of sweet pepper and Arabidopsis to feeding by $F$. occidentalis. The majority of DEGs from both plants having orthologues in the other plant species are differentially expressed in a species-specific manner: 718 up-regulated plus 405 down-regulated genes in sweet pepper and 393 up-regulated plus 324 down-regulated in Arabidopsis (Fig. 6). There was more overlap in orthologous genes up-regulated in both plant species (232, $24 \%$ of up-regulated pepper DEGs with Arabidopsis homologues) than in orthologous genes down-regulated in both species (37, $8 \%$ of down-regulated pepper DEGs with Arabidopsis homologues) (Fig. 6). Moreover, there is limited overlap in contrasting responses of orthologous genes, i.e. genes that are up-regulated in pepper and down-regulated in Arabidopsis (31) and vice-versa genes that are down-regulated in pepper and up-regulated in Arabidopsis (27). Genes from each subset are listed in Supplemental Data Set 7.

GO-term analysis was carried out to assess which processes have similar, specific or dissimilar regulation in the two plant species (Fig. 6). Common up-regulated DEGs (232) are associated with GO terms like "JA biosynthetic process" and several other defence-related processes, highlighting the important role of the JA pathway in both plants against WFT. DEGs in sweet pepper that have orthologues in Arabidopsis and that are up-regulated in pepper and not in Arabidopsis (718) are associated with GO terms like "isoprenoid biosynthetic process", "response to cadmium ion", whereas up-regulated genes in Arabidopsis that have orthologues in sweet pepper (393) are associated with GO terms like "response to stress", "response to biotic stimulus", "response to endogenous stimulus", reflecting defence responses that are specifically up-regulated in Arabidopsis and not in sweet pepper. In pepper, up-regulated DEGs not having orthologues in Arabidopsis (1079) exhibit an overrepresentation of the GO term "response to jasmonic acid", suggesting the existence of a unique downstream JA response in pepper. Moreover, among the common down-regulated genes (37), a high overrepresentation of genes involved in "spindle organization", "monopolar cell growth" and "transpiration" was recorded. Similarly, several common and unique GO terms for each subset of down-regulated genes are highlighted (Fig. 6). 


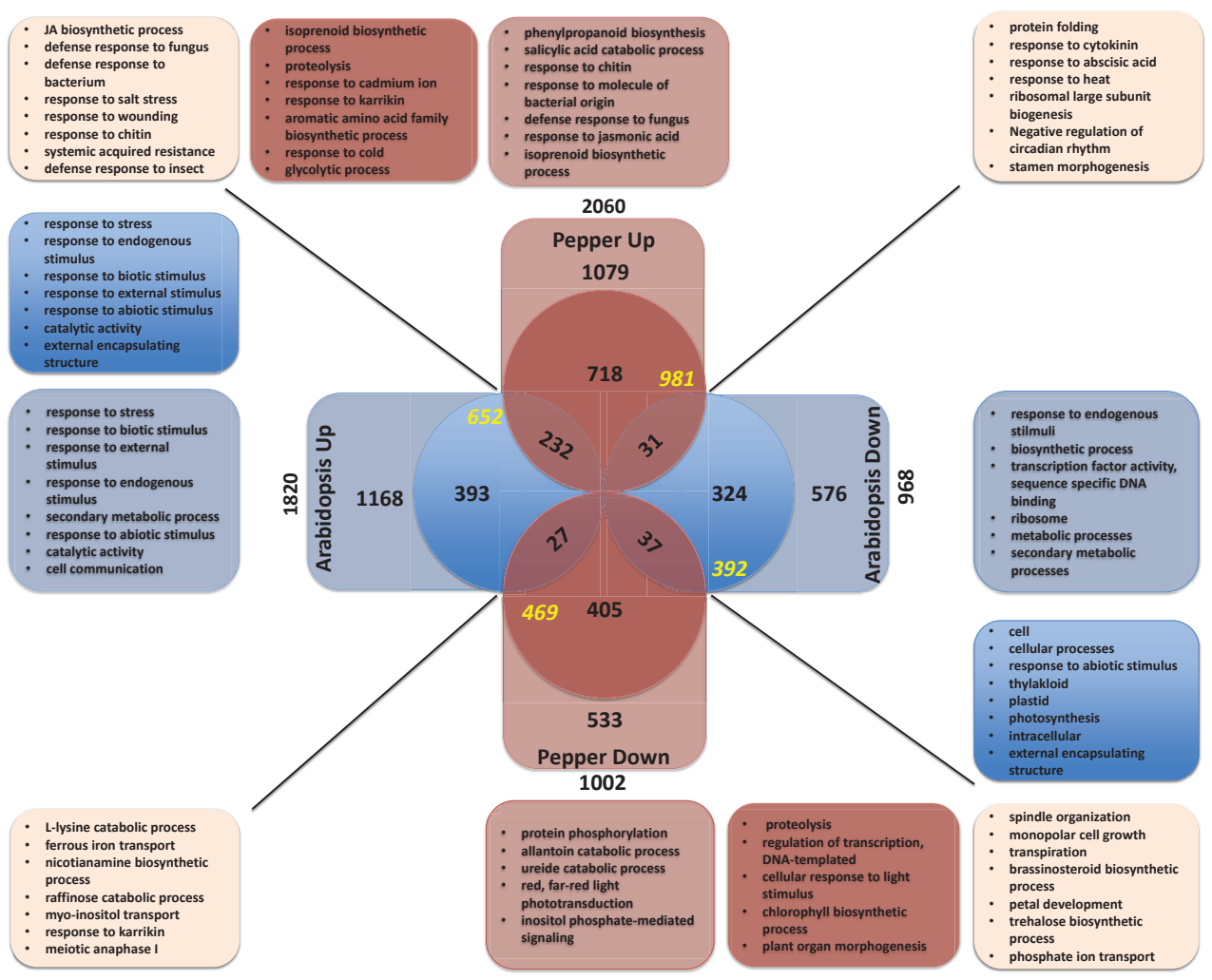

Figure 6. Venn diagram depicting conservation and uniqueness of induced defences in Arabidopsis and sweet pepper upon WFT feeding. Selected overrepresented GO terms are depicted for each subset of genes. The yellow numbers depicts orthologue number for each subset of genes.

\section{Discussion}

Through high-resolution temporal RNA-Seq and subsequent in-depth bioinformatic analysis, our study presents comprehensive temporal insights into sweet pepper transcriptomic responses to WFT feeding at an unprecedented level. This study shows that approximately $10 \%$ of the sweet pepper transcripts are involved in temporal transcriptional reprogramming upon thrips feeding. Most of this transcriptional reprogramming is initiated within $4 \mathrm{~h}$ after the onset of thrips feeding, with up-regulation exhibiting a faster time course than down-regulation. Down-regulated genes are especially involved in developmental processes and up-regulated genes are especially involved in defence mechanisms. The induced transcriptome depicts a dynamic expression pattern over time, consisting of 16 up-regulated gene clusters and comprising genes involved in several hormonal and secondary metabolite pathways. The in-depth bioin- 
formatic analysis also provides insight into the underlying regulators (TFs) and chronology of the transcriptomic rearrangement and biological processes affected. Moreover, a comparison with WFT-mediated rearrangement of the Arabidopsis transcriptome, indicates a core thrips response, which is early and involves processes related to JA biosynthesis and signalling, and a relatively large plant-species specific response, which includes isoprenoid biosynthetic process. There overlap in the transcriptomic responses of pepper and Arabidopsis to WFT is especially exhibited in JA-related processes.

\section{Transcriptional analysis of plant responses to insect herbivory}

Transcriptional responses to insect feeding have been carried out with microarrays and RNA-Seq for various plant species, but especially Arabidopsis. Full-genome microarray analyses have been carried out for Arabidopsis in response to feeding by caterpillars (De Vos et al., 2005; Ehlting et al., 2008; Bidart-Bouzat \& Kliebenstein, 2011; Appel et al., 2014; Davila Olivas et al., 2016; Kroes et al., 2017), aphids (De Vos et al., 2005; Bidart-Bouzat \& Kliebenstein, 2011; Kroes et al., 2017) or whiteflies (Kempema et al., 2007; Zhang et al., 2013). Full-genome microarray or RNA-Seq studies of responses of other plant species to herbivory have been carried out for e.g. caterpillars on wild cabbage (Broekgaarden et al., 2011), aphids on maize (Tzin et al., 2015) spider mites on tomato, maize, barley and grapevine (Martel et al., 2015; Diaz-Riquelme et al., 2016; Bui et al., 2018) or whiteflies on cabbage (Broekgaarden et al., 2018). Caterpillars have a biting-chewing feeding mode, removing sections of a leaf. Aphids and whiteflies have a piercing-sucking feeding mode, and ingest phloem contents. Spider mites have delicate piercing stylets, which they insert in the plant to pierce mesophyll cells to ingest their contents. Thrips also feed on mesophyll cells. They damage a group of cells with their mouthparts and then suck up the contents of the opened cells with their stylets (Steenbergen et al., 2018). To the best of our knowledge there are two published studies that have made a full-genome transcriptomic analysis of plant responses to thrips: alfalfa response to feeding by Odontothrips loti (Tu et al., 2018) (RNA-Seq analysis, only one time point) and Arabidopsis response to Frankliniella occidentalis (De Vos et al., 2005) (microarray analysis, two time points spread over 24h). Although Arabidopsis also shows a strong representation of genes involved in JA-related processes among the up-regulated genes (De Vos et al., 2005). The SA pathway seems especially up-regulated in alfalfa (Tu et al., 2018).

Most transcriptomic studies of plant responses to insect feeding have assessed the response at one or two time points that often span a time period of $24 \mathrm{~h}$ or longer (De Vos et al., 2005; Ehlting et al., 2008; Bidart-Bouzat \& Kliebenstein, 2011; Zhang et al., 2013; Appel et al., 2014; Diaz-Riquelme et al., 2016; Kroes et al., 2017; Broekgaarden et al., 2018; Tu et al., 2018). An exception are studies by Coolen et al. (2016) who included 4 time points during $24 \mathrm{~h}$ of feeding by caterpillars on Arabidopsis and 
Durrant et al. (2017) who assessed the transcriptomic response of wild tobacco to the application of oral secretion of the caterpillar Manduca sexta at 6 time points ranging from 0.5 to 13 hours since treatment In the latter study no further feeding damage was done throughout the 13 hours of the experiment. The present study is the first to provide a detailed high-resolution analysis of the early transcriptional response of a plant to a cell-content feeding insect herbivore, and thus provides a valuable resource for investigating this complex interaction. Our data show that with 6 time points in the first $8 \mathrm{~h}$ of thrips infestation a total 23 clusters of transcriptional patterns can be distinguished, indicative of the complexity of the transcriptional response to continuing infliction of insect herbivory.

\section{Temporal transcriptomic dynamics of sweet pepper to WFT feeding}

Most of the temporal transcriptomic plant responses to insect herbivory are studied with limited time points and not possessing control or mock samples for each time point (De Vos et al., 2005; Ehlting et al., 2008; Bidart-Bouzat \& Kliebenstein, 2011; Zhang et al., 2013; Appel et al., 2014; Diaz-Riquelme et al., 2016; Kroes et al., 2017; Broekgaarden et al., 2018; Tu et al., 2018). To obtain accurate insights into temporal transcriptome dynamics, it is not only crucial to harvest more time points, but it is also vital to harvest the control or mock samples for each time point (Breeze et al., 2011; Windram et al., 2012; Lewis et al., 2015; Hickman et al., 2017). Harvesting at a limited number of time points will provide a low-resolution temporal transcriptome portrait of the plant response. Moreover, when control or mock samples are not included for each time point an effect of circadian rhythm (Fig. 1A) is not compensated for in the temporal differential gene expression analysis, generating false positives. Therefore, in our study, together with harvesting more time points, inclusion of control samples for each time point was an important feature. Furthermore, overall, sweet pepper responded with a temporally dynamic transcriptome to WFT feeding, including more up-regulated (2060) genes than down-regulated (1002) genes. This temporally dynamic transcriptome shows that numbers of genes induced or repressed vary among different time points, indicating major switch points for up- and down-regulated defence mechanisms at different time scales. This suggests that harvesting tissue at fewer time points bears the risk of missing important temporal dynamics in the transcriptome response.

\section{Role of phytohormones and secondary metabolites in sweet pepper against WFT}

Besides the role of $\mathrm{JA}$, relatively little knowledge on other defence components against thrips resistance is available (Maharijaya et al., 2012). JA is a major phytohormone in regulating induced plant defences against thrips (De Vos et al., 2005; Abe et al., 2008). For example, $69 \%$ of differentially expressed genes in Arabidopsis were JA-re- 
lated (De Vos et al., 2005). JA-signaling-impaired tomato and pepper plants show enhanced susceptibility to thrips (Li et al., 2002; Escobar-Bravo et al., 2017; Sarde et al., 2018a). Induction of the whole JA cascade in our RNA-Seq dataset validates the conservation of JA-regulated defences in sweet pepper against thrips (Sarde et al., 2018a). Some of the JA-regulated proteins like proteinase inhibitors (e.g. encoded by CaPIN II) and Arginase (CaARG1) are known for antidigestive effects in WFT (Outchkourov et al., 2004) or other arthropods (Chen et al., 2004). Likewise, up-regulation of several genes associated with the ET pathway may be indicative of synergism between the JA and ET pathways in defence of sweet pepper against thrips as has been shown for other biotic stresses in Arabiopsis (Pieterse et al., 2009). SA biosynthesis in plants occurs either via the PAL or ICS pathway (Chen et al., 2009). Up-regulation of several homologues of CaPAL and down-regulation of CalCS and the SA-responsive CaPR1 (Fig. $5 \mathrm{C}$ ) gene suggest a suppression of SA pathway (at least until 8h), upon thrips feeding. PAL genes are also known to be involved in the biosynthesis of phenylpropanoids and flavonoids and their induction does not necessarily mean an induction of the SA pathway. Sarde et al. (2018a) also showed that CaPR1 is induced later i.e. post $10 \mathrm{~h}$ of thrips feeding, suggesting later activation the SA pathway. Furthermore, up-regulation of phenylpropanoid and flavonoid biosynthetic genes and their homologs (Ca4CL1, CaC4H), together with CaPAL homologues, suggests a defensive role of these pathways against thrips. Moreover, plants also activate indirect defence by emitting VOCs upon herbivory feeding to attract their natural enemies (Mithofer \& Boland, 2012; Dicke, 2015). The blend of VOCs emitted mainly comprises of GLVs, methyl salicylate (MeSA) and terpenoids (Dudareva et al., 2006; Mumm et al., 2008). Terpenoid biosynthesis occurs via the cytosolic MVA (mevalonate) or plastidal MEP (methylerythritol 4-phosphate) pathways (Vranova et al., 2013). In this study, together with many MVA and MEP pathway genes, the genes involved in biosynthesis of MeSA (CaSAMT), GLVs (CaLOX7) (Sarde et al., 2018b) and terpenoids (Fig. $5 \mathrm{D})$ are induced. This suggests the emission of a VOC blend similar to that induced by another cell-content feeder, the spider mite $T$. urticae in sweet pepper (Van Den Boom et al., 2004).

\section{Cluster analysis identifies major regulators in sweet pepper}

Several TF families regulate the transcriptional reprogramming of plants in a stress-specific manner (Breeze et al., 2011; Windram et al., 2012; Hickman et al., 2017; Jin et al., 2017). Upon thrips feeding, the TF families ERF, MYB, NAC, bHLH and WRKY appeared to be major regulators in modulating the majority of thrips up-regulated genes. Recently, Hickman et al. (2017) showed that the ERF, MYB and bHLH TF families are major regulators of transcriptome reprogramming in response to exogenous MeJA application. This suggests that regulation of JA-signalling in Arabidopsis and sweet pepper is conserved. In contrast, WRKY TFs, known to regu- 
late SA-mediated responses (Pandey \& Somssich, 2009; Rushton et al., 2010) are overrepresented among the up-regulated genes (Fig. 3A), suggesting an induction of SA responses upon thrips feeding. In contrast, the down-regulation of SA biosynthetic (isochorismate synthase (CalCS)) and SA-responsive (CaPR1) gene suggests suppression of the SA pathway (at least until 8h) upon thrips feeding. Possibly, the up-regulated WRKY TFs regulate phenylpropanoid, flavonoid or terpene biosynthesis (Schluttenhofer \& Yuan, 2015) or SA responses independent of the isochorismate pathway are activated (Chen et al., 2009). Furthermore, in the TF motif analysis, binding sites of bHLH TFs, known to be involved in JA signalling (Goossens et al., 2017), appeared to be enriched in several clusters (Fig. 3B), suggesting that bHLH TFs extensively regulate thrips-induced JA-regulated genes. In contrast, ERFs appeared to be enriched in a few clusters suggesting that they regulate only a small component of the transcriptional response to thrips. Likewise, enrichment of WRKY TFs in clusters overrepresented with SA-responses consolidates its role as pivotal regulator of SA-response pathways (Pandey \& Somssich, 2009).

\section{Sequential activation of phytohormones and secondary metabolites}

In plant-herbivore interactions, phytohormones are induced early (timescale of minutes to hours) resulting in an activation and regulation of the downstream transcriptome (Maffei et al., 2007). Many studies have shown a reprogramming of the transcriptome occurring in plants with time in response to different stresses (Breeze et al., 2011; Windram et al., 2012; Bechtold et al., 2016; Hickman et al., 2017). This temporal transcriptome reprogramming has consequences for the activation of different metabolic processes (timescale of hours to days) involved in plant defence (Maffei et al., 2007). In the chronology analysis, at the $2 \mathrm{~h}$ time point, $\mathrm{GO}$ terms like "response to JA", and "response to wounding" are overrepresented. Similarly, genes involved in JA biosynthesis (CaLOX2, CaAOS, CaAOC, CaOPR3, CaJAZ1, CaJAZ3, CaMYC2) and ET biosynthesis (CaSAMS2, CaACO3) (Fig. 4) are differentially expressed for the first time (ftode) at this time point, indicative of activation of the JA and ET hormonal pathways within $2 \mathrm{~h}$ of thrips feeding. At the $4 \mathrm{~h}$ time point, overrepresentation of $\mathrm{GO}$ terms like "phenylpropanoid biosynthetic process", "GLVs biosynthetic process" and activation (ftode) of major genes involved in biosynthesis of MeSA (CaSAMT) and terpenoids via MVA and MEP (CaAACT, CaHMGR, CaMK, CaHDR, CaMPDC, CaIPPI, CaGLS) pathways indicates that the induced biosynthesis of these secondary metabolites is initiated at this time point. The major regulator of the most abundant volatile induced in sweet pepper by spider mites, the homoterpene $(E, E)$-4,8,12-trimethyltrideca-1,3,7,11-tetraene (TMTT), CaGGPPS, is activated earlier at $3 \mathrm{~h}$ time point (Fig. $5 \mathrm{~F})$. This fits to the fact that VOCs such as ester methyl salicylate (MeSA), monoterpenes myrcene and $\beta$-ocimene, the homoterpene TMTT, and the sesquiterpene $(E, E)$-a- farnesene are emitted hours after herbivorous insect infestation (Erb et al., 
2015; Aljbory \& Chen, 2018).

\section{Majority of induced defences are plant-species specific against WFT}

To gain more insight in conservation and diversification of induced defence mechanisms against WFT between Arabidopsis and sweet pepper, we implemented a comparative transcriptomic analysis. From this analysis it appears that more genes, up- as well as down-regulated DEGs are specific for each of the two plant species than common for both of them. There was more overlap in up-regulated DEGs (232) than in down-regulated DEGs (37), which indicates that up-regulated responses such as induced defences are more conserved than down-regulated responses, which concern plant growth and development. The prominent association of a common subset of up-regulated DEGs (232) with the GO term "JA biosynthetic process", comprising mainly JA biosynthetic and signalling genes (CaLOX2, CaAOS, CaAOC, CaOPR3, CaACX1, CaJAZ1, CaJAZ3, CaMYC2, CaJMT) together with other genes involved in ET biosynthesis (CaSAMS2), ET response (CaERF1, CaERF5), phenylpropanoid biosynthesis $(\mathrm{CaC} 4 \mathrm{H})$ and indirect defence mechanism (CaSAMT, CaGLS, CaLOX7), indicates a prominent conservation of the JA pathway with other defence mechanisms in Arabidopsis and sweet pepper. Furthermore, the common up-regulated DEGs (232) of pepper are associated with the GO term "JA biosynthetic process", whereas the non-orthologous DEGs (1079) from pepper are associated with the GO term "response to JA". This suggests that the JA biosynthetic pathway is conserved, whereas the response to this phytohormone is diverged between the two plant species. One of the JA responsive pathways in Arabidopsis is the glucosinolate pathway, resulting in secondary metabolites that are specific for brassicaceous plants like Arabidopsis and not sweet pepper, Moreover, DEGs possessing orthologues in pepper (718 upand 405 down-regulated) and in Arabidopsis (393 up- and 324 down-regulated), that are assumed to have similar or identical functions in both plants, are differentially expressed in only one species. Likewise, the DEGs not possessing orthologues, in pepper (1079 up- and 533 down-regulated) and in Arabidopsis (1168 up- and 576 down-regulated), are also species-specifically differentially expressed. The species-specific differential expression of orthologues possessing and non-orthologue possessing DEGs explicitly indicates that the majority of the transcriptome response of Arabidopsis and pepper to WFT feeding is plant species-specific. Broadly, similar observations were reported to each subset of DEGs between Arabidopsis and tomato microarray-based transcriptome data generated in response to another cell-content feeder, the spider-mite T. urticae (Martel et al., 2015). Taken together, JA is found to be a prominently conserved pathway in Arabidopsis and pepper responses to WFT feeding, with the majority of the transcriptome response being species-specific. 


\section{Conclusion}

In conclusion, this study provides high-resolution information on the temporal transcriptomic response of sweet pepper to WFT feeding. Through in-depth bioinformatic analysis, this study captured the temporal transcriptional reprogramming and its regulators and chronology of underlying defence mechanisms. Moreover, a comparison with the WFT-induced Arabidopsis transcriptome shows more commonalities in induced responses, with a prominent involvement of JA biosynthesis and signalling, than for suppressed responses. Thus, this detailed, in-depth in-silico analysis provides important insights into the dynamic and complex response of pepper plants to infestation with a cell-content feeding herbivorous insect.

\section{Acknowledgements}

We thank Keygene for RNA sequencing, Gerben Messelink for help with the culture of thrips, Wageningen University \& Research experimental farm (Unifarm) for rearing the plants, and colleagues from the Laboratory of Entomology (Wageningen University) and the Plant-Microbe Interactions group (Utrecht University) for help in sampling leaf tissues for RNA-Seq analysis. This work is part of the Perspective programme Green Defences Against Pests that is (partly) financed by the Netherlands Organisation for Scientific Research (NWO, grant no. 13555) and partly by the breeding companies Keygene, Syngenta and East-West Seeds through NWO.

\section{References}

Abe H, Ohnishi J, Narusaka M, Seo S, Narusaka Y, Tsuda S, Kobayashi M. 2008. Function of jasmonate in response and tolerance of Arabidopsis to thrip feeding. Plant Cell Physiology 49(1): 68-80.

Abe H, Shimoda T, Ohnishi J, Kugimiya S, Narusaka M, Seo S, Narusaka Y, Tsuda S, Kobayashi M. 2009. Jasmonate-dependent plant defense restricts thrips performance and preference. BMC Plant Biology 9: 97.

Aljbory Z, Chen MS. 2018. Indirect plant defense against insect herbivores: a review. Insect Science 25(1): 2-23.

Anders S, Pyl PT, Huber W. 2015. HTSeq-a Python framework to work with high-throughput sequencing data. Bioinformatics 31(2): 166-169.

Appel HM, Fescemyer H, Ehlting J, Weston D, Rehrig E, Joshi T, Xu D, Bohlmann J, Schultz J. 2014. Transcriptional responses of Arabidopsis thaliana to chewing and sucking insect herbivores. Frontiers in Plant Science 5: 565.

Bechtold U, Penfold CA, Jenkins DJ, Legaie R, Moore JD, Lawson T, Matthews JSA, Vialet-Chabrand SRM, Baxter L, Subramaniam S, Hickman R, Florance H, Sambles C, Salmon DL, Feil R, Bowden L, Hill C, Baker NR, Lunn JE, Finkenstadt B, Mead A, Buchanan-Wollaston V, Beynon J, Rand DA, Wild DL, Denby KJ, Ott S, Smirnoff N, 
Mullineaux PM. 2016. Time-series transcriptomics reveals that AGAMOUS-LIKE22 affects primary metabolism and developmental processes in drought-stressed Arabidopsis. Plant Cell 28(2): 345-366.

Bidart-Bouzat MG, Kliebenstein D. 2011. An ecological genomic approach challenging the paradigm of differential plant responses to specialist versus generalist insect herbivores. Oecologia 167(3): 677-689.

Bolger AM, Lohse M, Usadel B. 2014. Trimmomatic: a flexible trimmer for Illumina sequence data. Bioinformatics 30(15): 2114-2120.

Bonaventure G. 2012. Perception of insect feeding by plants. Plant Biology 14(6): 872-880.

Bonnet C, Lassueur S, Ponzio C, Gols R, Dicke M, Reymond P. 2017. Combined biotic stresses trigger similar transcriptomic responses but contrasting resistance against a chewing herbivore in Brassica nigra. BMC Plant Biology 17: 127.

Breeze E, Harrison E, McHattie S, Hughes L, Hickman R, Hill C, Kiddle S, Kim YS, Penfold CA, Jenkins D, Zhang CJ, Morris K, Jenner C, Jackson S, Thomas B, Tabrett A, Legaie R, Moore JD, Wild DL, Ott S, Rand D, Beynon J, Denby K, Mead A, Buchanan-Wollaston V. 2011. High-resolution temporal profiling of transcripts during Arabidopsis leaf senescence reveals a distinct chronology of processes and regulation. Plant Cell 23(3): 873-894.

Broekgaarden C, Pelgrom KTB, Bucher J, van Dam NM, Grosser K, Pieterse CMJ, van Kaauwen M, Steenhuis G, Voorrips RE, de Vos M, Vosman B, Worrich A, van Wees SCM. 2018. Combining QTL mapping with transcriptome and metabolome profiling reveals a possible role for ABA signaling in resistance against the cabbage whitefly in cabbage. PLoS One 13(11): e0206103.

Broekgaarden C, Voorrips RE, Dicke M, Vosman B. 2011. Transcriptional responses of Brassica nigra to feeding by specialist insects of different feeding guilds. Insect Science 18(3): 259-272.

Bui H, Greenhalgh R, Ruckert A, Gill GS, Lee S, Ramirez RA, Clark RM. 2018. Generalist and specialist mite herbivores induce similar defense responses in maize and barley but differ in susceptibility to benzoxazinoids. Frontiers in Plant Science 9: 1222.

Chen H, McCaig BC, Melotto M, He SY, Howe GA. 2004. Regulation of plant arginase by wounding, jasmonate, and the phytotoxin coronatine. Journal of Biological Chemistry 279(44): 45998-46007.

Chen Z, Zheng Z, Huang J, Lai Z, Fan B. 2009. Biosynthesis of salicylic acid in plants. Plant Signaling and Behavior 4(6): 493-496.

Coolen S, Proietti S, Hickman R, Davila Olivas NH, Huang PP, Van Verk MC, Van Pelt JA, Wittenberg AHJ, De Vos M, Prins M, Van Loon JJA, Aarts MGM, Dicke M, Pieterse CMJ, Van Wees SCM. 2016. Transcriptome dynamics of Arabidopsis during sequential biotic and abiotic stresses. Plant Journal 86(3): 249-267.

Davila Olivas $\mathrm{NH}$, Coolen S, Huang PP, Severing E, van Verk MC, Hickman R, Wittenberg AHJ, de Vos M, Prins M, van Loon JJA, Aarts MGM, van Wees SCM, Pieterse CMJ, Dicke M. 2016. Effect of prior drought and pathogen stress on Arabidopsis transcriptome changes to caterpillar herbivory. New Phytologist 210(4): 1344-1356.

De Vos M, Van Oosten VR, Van Poecke RMP, Van Pelt JA, Pozo MJ, Mueller MJ, Buchala AJ, Metraux JP, Van Loon LC, Dicke M, Pieterse CMJ. 2005. Signal signature and transcriptome changes of Arabidopsis during pathogen and insect attack. Molecular Plant-Microbe Interactions 18(9): 923-937.

Diaz-Riquelme J, Zhurov V, Rioja C, Perez-Moreno I, Torres-Perez R, Grimplet J, Car- 
bonell-Bejerano P, Bajda S, Van Leeuwen T, Martinez-Zapater JM, Grbic M, Grbic V. 2016. Comparative genome-wide transcriptome analysis of Vitis vinifera responses to adapted and non-adapted strains of two-spotted spider mite, Tetranyhus urticae. BMC Genomics 17: 74.

Dicke M. 2009. Behavioural and community ecology of plants that cry for help. Plant Cell and Environment 32(6): 654-665.

Dicke M. 2015. Herbivore-induced plant volatiles as a rich source of information for arthropod predators: Fundamental and applied aspects. Journal of the Indian Institute of Science 95(1): 35-42.

Dicke M, Vanbeek TA, Posthumus MA, Bendom N, Vanbokhoven H, Degroot AE. 1990. Isolation and identification of volatile kairomone that affects acarine predator-prey interactions - Involvement of host plant in its production. Journal of Chemical Ecology 16(2): 381-396.

Dudareva N, Negre F, Nagegowda DA, Orlova I. 2006. Plant volatiles: Recent advances and future perspectives. Critical Reviews in Plant Sciences 25(5): 417-440.

Duran-Flores D, Heil M. 2016. Sources of specificity in plant damaged-self recognition. Current Opinion in Plant Biology 32: 77-87.

Durrant M, Boyer J, Zhou WW, Baldwin IT, Xu SQ. 2017. Evidence of an evolutionary hourglass pattern in herbivory-induced transcriptomic responses. New Phytologist 215(3): 1264-1273.

Ehlting J, Chowrira SG, Mattheus N, Aeschliman DS, Arimura GI, Bohlmann J. 2008. Comparative transcriptome analysis of Arabidopsis thaliana infested by diamond back moth (Plutella xylostella) larvae reveals signatures of stress response, secondary metabolism, and signalling. BMC Genomics 9: 154.

Erb M, Meldau S, Howe GA. 2012. Role of phytohormones in insect-specific plant reactions. Trends in Plant Science 17(5): 250-259.

Erb M, Veyrat N, Robert CAM, Xu H, Frey M, Ton J, Turlings TCJ. 2015. Indole is an essential herbivore-induced volatile priming signal in maize. Nature Communications 6: 6273.

Escobar-Bravo R, Klinkhamer PGL, Leiss KA. 2017. Induction of jasmonic acid-associated defenses by thrips alters host suitability for conspecifics and correlates with increased trichome densities in Tomato. Plant and Cell Physiology 58(3): 622-634.

Franco-Zorrilla JM, Lopez-Vidriero I, Carrasco JL, Godoy M, Vera P, Solano R. 2014. DNA-binding specificities of plant transcription factors and their potential to define target genes. Proceedings of the National Academy of Sciences of the United States of America 111(6): 2367-2372.

Gershenzon J, Dudareva N. 2007. The function of terpene natural products in the natural world. Nature Chemical Biology 3(7): 408-414.

Goossens J, Mertens J, Goossens A. 2017. Role and functioning of bHLH transcription factors in jasmonate signalling. Journal of Experimental Botany 68(6): 1333-1347.

Grant CE, Bailey TL, Noble WS. 2011. FIMO: scanning for occurrences of a given motif. Bioinformatics 27(7): 1017-1018.

Heard NA. 2011. Iterative reclassification in agglomerative clustering. J. Comput. Graph. Stat. 20: 920-936.

Heard NA, Holmes CC, Stephens DA. 2006. A quantitative study of gene regulation involved in the immune response of anopheline mosquitoes: An application of bayesian hierarchical clustering of curves. J. Am. Stat. Assoc. 101: 18-29. 
Heidel-Fischer HM, Musser RO, Vogel H 2014. Plant transcriptomic responses to herbivory. Annu. Plant Rev. : 47, pp 155-196.

Hickman R, Van Verk MC, Van Dijken AJH, Mendes MP, Vroegop-Vos IA, Caarls L, Steenbergen M, Van der Nagel I, Wesselink GJ, Jironkin A, Talbot A, Rhodes J, De Vries M, Schuurink RC, Denby K, Pieterse CMJ, Wees SCM. 2017. Architecture and dynamics of the jasmonic acid gene regulatory network. Plant Cell 29(9): 2086-2105.

Hiruma K, Fukunaga S, Bednarek P, Pislewska-Bednarek M, Watanabe S, Narusaka Y, Shirasu K, Takano Y. 2013. Glutathione and tryptophan metabolism are required for Arabidopsis immunity during the hypersensitive response to hemibiotrophs. Proceedings of the National Academy of Sciences of the United States of America 110(23): 9589-9594.

Howe GA, Jander G. 2008. Plant immunity to insect herbivores. Annual Review of Plant Biology 59: 41-66.

Jin JP, Tian F, Yang DC, Meng YQ, Kong L, Luo JC, Gao G. 2017. PlantTFDB 4.0: toward a central hub for transcription factors and regulatory interactions in plants. Nucleic Acids Research 45(D1): D1040-D1045.

Kang S, Back K. 2006. Enriched production of N-hydroxycinnamic acid amides and biogenic amines in pepper (Capsicum annuum) flowers. Scientia Horticulturae 108(3): 337-341.

Kempema LA, Cui X, Holzer FM, Walling LL. 2007. Arabidopsis transcriptome changes in response to phloem-feeding silverleaf whitefly nymphs. Similarities and distinctions in responses to aphids. Plant Physiology 143(2): 849-865.

Kessler A, Baldwin IT. 2002. Plant responses to insect herbivory: The emerging molecular analysis. Annual Review of Plant Biology 53: 299-328.

Kim D, Pertea G, Trapnell C, Pimentel H, Kelley R, Salzberg SL. 2013. TopHat2: accurate alignment of transcriptomes in the presence of insertions, deletions and gene fusions. Genome Biology 14(4): R36.

Kim S, Park M, Yeom SI, Kim YM, Lee JM, Lee HA, Seo E, Choi J, Cheong K, Kim KT, Jung K, Lee GW, Oh SK, Bae C, Kim SB, Lee HY, Kim SY, Kim MS, Kang BC, Jo YD, Yang HB, Jeong HJ, Kang WH, Kwon JK, Shin C, Lim JY, Park JH, Huh JH, Kim JS, Kim BD, Cohen O, Paran I, Suh MC, Lee SB, Kim YK, Shin Y, Noh SJ, Park J, Seo YS, Kwon SY, Kim HA, Park JM, Kim HJ, Choi SB, Bosland PW, Reeves G, Jo SH, Lee BW, Cho HT, Choi HS, Lee MS, Yu Y, Do Choi Y, Park BS, van Deynze A, Ashrafi H, Hill T, Kim WT, Pai HS, Ahn HK, Yeam I, Giovannoni JJ, Rose JKC, Sorensen I, Lee SJ, Kim RW, Choi IY, Choi BS, Lim JS, Lee YH, Choi D. 2014. Genome sequence of the hot pepper provides insights into the evolution of pungency in Capsicum species. Nature Genetics 46(3): 270-+.

Klopfenstein DV, Zhang LS, Pedersen BS, Ramirez F, Vesztrocy AW, Naldi A, Mungall CJ, Yunes JM, Botvinnik O, Weigel M, Dampier W, Dessimoz C, Flick P, Tang HB. 2018. GOATOOLS: A Python library for gene ontology analyses. Scientific Reports 8: 10872.

Kroes A, Broekgaarden C, Uribe MC, May S, van Loon JJA, Dicke M. 2017. Brevicoryne brassicae aphids interfere with transcriptome responses of Arabidopsis thaliana to feeding by Plutella xylostella caterpillars in a density-dependent manner. Oecologia 183(1): 107-120.

Labandeira C. 2007. The origin of herbivory on land: Initial patterns of plant tissue consumption by arthropods. Insect Science 14(4): 259-275.

Lewis LA, Polanski K, de Torres-Zabala M, Jayaraman S, Bowden L, Moore J, Penfold CA, Jenkins DJ, Hill C, Baxter L, Kulasekaran S, Truman W, Littlejohn G, Prusinska J, Mead A, Steinbrenner J, Hickman R, Rand D, Wild DL, Ott S, Buchanan-Wollaston V, Smirnoff N, Beynon J, Denby K, Grant M. 2015. Transcriptional dynamics driving 
MAMP-triggered immunity and pathogen effector-mediated immunosuppression in Arabidopsis leaves following infection with Pseudomonas syringae pv tomato DC3000. Plant Cell 27(11): 3038-3064.

Li CY, Williams MM, Loh YT, Lee GI, Howe GA. 2002. Resistance of cultivated tomato to cell content-feeding herbivores is regulated by the octadecanoid-signaling pathway. Plant Physiology 130(1): 494-503.

Love MI, Anders S, Kim V, Huber W. 2015. RNA-Seq workflow: gene-level exploratory analysis and differential expression. F1000Res 4: 1070.

Love MI, Huber W, Anders S. 2014. Moderated estimation of fold change and dispersion for RNA-Seq data with DESeq2. Genome Biology 15(12): 550.

Maffei ME, Mithofer A, Boland W. 2007. Before gene expression: early events in plant-insect interaction. Trends in Plant Science 12(7): 310-316.

Maharijaya A, Vosman B, Verstappen F, Steenhuis-Broers G, Mumm R, Purwito A, Visser RGF, Voorrips RE. 2012. Resistance factors in pepper inhibit larval development of thrips (Frankliniella occidentalis). Entomologia Experimentalis Et Applicata 145(1): 62-71.

Maris PC, Joosten NN, Peters D, Goldbach RW. 2003. Thrips resistance in pepper and its consequences for the acquisition and inoculation of Tomato spotted wilt virus by the western flower thrips. Phytopathology 93(1): 96-101.

Martel C, Zhurov V, Navarro M, Martinez M, Cazaux M, Auger P, Migeon A, Santamaria ME, Wybouw N, Diaz I, Van Leeuwen T, Navajas M, Grbic M, Grbic V. 2015. Tomato whole genome transcriptional response to Tetranychus urticae identifies divergence of spider mite-induced responses between Tomato and Arabidopsis. Molecular Plant-Microbe Interactions 28(3): 343-361.

McCormick AC, Unsicker SB, Gershenzon J. 2012. The specificity of herbivore-induced plant volatiles in attracting herbivore enemies. Trends in Plant Science 17(5): 303-310.

Mithofer A, Boland W. 2008. Recognition of herbivory-associated molecular patterns. Plant Physiology 146(3): 825-831.

Mithofer A, Boland W. 2012. Plant defense against herbivores: Chemical aspects. Annual Review of Plant Biology 63: 431-450.

Mouden S, Sarmiento KF, Klinkhamer PGL, Leiss KA. 2017. Integrated pest management in western flower thrips: past, present and future. Pest Management Science 73(5): 813822.

Mumm R, Posthumus MA, Dicke M. 2008. Significance of terpenoids in induced indirect plant defence against herbivorous arthropods. Plant Cell and Environment 31(4): 575-585.

Omer AD, Granett J, Karban R, Villa EM. 2001. Chemically-induced resistance against multiple pests in cotton. International Journal of Pest Management 47(1): 49-54.

Outchkourov NS, de Kogel WJ, Wiegers GL, Abrahamson M, Jongsma MA. 2004. Engineered multidomain cysteine protease inhibitors yield resistance against western flower thrips (Frankliniella occidentalis) in greenhouse trials. Plant Biotechnology Journal 2(5): 449-458.

Pandey SP, Somssich IE. 2009. The role of WRKY transcription factors in plant immunity. Plant Physiology 150(4): 1648-1655.

Pieterse CMJ, Leon-Reyes A, Van der Ent S, Van Wees SCM. 2009. Networking by small-molecule hormones in plant immunity. Nature Chemical Biology 5(5): 308-316.

Pieterse CMJ, Van der Does D, Zamioudis C, Leon-Reyes A, Van Wees SCM. 2012. Hor- 
monal modulation of plant immunity. Annual Review of Cell and Developmental Biology 28: 489-521.

Portales-Casamar E, Thongjuea S, Kwon AT, Arenillas D, Zhao XB, Valen E, Yusuf D, Lenhard B, Wasserman WW, Sandelin A. 2010. JASPAR 2010: the greatly expanded open-access database of transcription factor binding profiles. Nucleic Acids Research 38: D105-D110.

Qin C, Yu CS, Shen YO, Fang XD, Chen L, Min JM, Cheng JW, Zhao SC, Xu M, Luo Y, Yang YL, Wu ZM, Mao LK, Wu HY, Ling-Hu CY, Zhou HK, Lin HJ, Gonzalez-Morales S, Trejo-Saavedra DL, Tian H, Tang X, Zhao MJ, Huang ZY, Zhou AW, Yao XM, Cui JJ, Li WQ, Chen Z, Feng YQ, Niu YC, Bi SM, Yang XW, Li WP, Cai HM, Luo XR, Montes-Hernandez S, Leyva-Gonzalez MA, Xiong ZQ, He XJ, Bai LJ, Tan S, Tang XQ, Liu D, Liu JW, Zhang SX, Chen MS, Zhang L, Zhang L, Zhang YC, Liao WQ, Zhang Y, Wang M, Lv XD, Wen B, Liu HJ, Luan HM, Zhang YG, Yang S, Wang XD, Xu JH, Li XQ, Li SC, Wang JY, Palloix A, Bosland PW, Li YR, Krogh A, Rivera-Bustamante RF, Herrera-Estrella L, Yin Y, Yu JP, Hu KL, Zhang ZM. 2014. Whole-genome sequencing of cultivated and wild peppers provides insights into Capsicum domestication and specialization. Proceedings of the National Academy of Sciences of the United States of America 111(14): 5135-5140.

Rushton PJ, Somssich IE, Ringler P, Shen QXJ. 2010. WRKY transcription factors. Trends in Plant Science 15(5): 247-258.

Sarde SJ, Bouwmeester K, Venegas-Molina J, David A, Boland W, Dicke M. 2018a. Involvement of sweet pepper CaLOX2 in jasmonate-dependent induced defence against Western flower thrips. Journal of Integrative Plant Biology: 10.1111/jipb.12742.

Sarde SJ, Kumar A, Remme RN, Dicke M. 2018b. Genome-wide identification, classification and expression of lipoxygenase gene family in pepper. Plant Molecular Biology: 10.1007/ s11103-11018-10785-y.

Schluttenhofer C, Yuan L. 2015. Regulation of specialized metabolism by WRKY transcription factors. Plant Physiology 167(2): 295-306.

Schoonhoven LM, Van Loon JJA, Dicke M. 2005. Insect plant biology. Oxford University Press, Oxford: $421 \mathrm{pp}$.

Selig P, Keough S, Nalam VJ, Nachappa P. 2016. Jasmonate-dependent plant defenses mediate soybean thrips and soybean aphid performance on soybean. Arthropod-Plant Interactions 10(4): 273-282.

Shannon P, Markiel A, Ozier O, Baliga NS, Wang JT, Ramage D, Amin N, Schwikowski B, Ideker T. 2003. Cytoscape: A software environment for integrated models of biomolecular interaction networks. Genome Research 13(11): 2498-2504.

Shipp JL, Hao X, Papadopoulos AP, Binns MR. 1998. Impact of western flower thrips (Thysanoptera : Thripidae) on growth, photosynthesis and productivity of greenhouse sweet pepper. Scientia Horticulturae 72(2): 87-102.

Stam JM, Kroes A, Li YH, Gols R, van Loon JJA, Poelman EH, Dicke M. 2014. Plant interactions with multiple insect herbivores: From community to genes. Annual Review of Plant Biology 65: 689-713.

Steenbergen M, Abd-el-Haliem A, Bleeker P, Dicke M, Escobar-Bravo R, Cheng G, Haring MA, Kant MR, Kappers I, Klinkhamer PGL, Leiss KA, Legarrea S, Macel M, Mouden S, Pieterse CMJ, Sarde SJ, Schuurink RC, De Vos M, Van Wees SCM, Broekgaarden C. 2018. Thrips advisor: exploiting thrips-induced defences to combat pests on crops. Journal of Experimental Botany 69(8): 1837-1848.

Steiner MY. 1990. Determining population characteristics and sampling procedures for the 
western flower thrips (Thysanoptera, Thripidae) and the predatory mite Amblyseius-cucumeris (Acari, Phytoseiidae) on greenhouse cucumber. Environmental Entomology 19(5): 1605-1613.

Thaler JS, Stout MJ, Karban R, Duffey SS. 2001. Jasmonate-mediated induced plant resistance affects a community of herbivores. Ecological Entomology 26(3): 312-324.

Tu XB, Liu ZK, Zhang ZH. 2018. Comparative transcriptomic analysis of resistant and susceptible alfalfa cultivars (Medicago sativa L.) after thrips infestation. BMC Genomics 19: 10.1186/s12864-018-4495-2.

Tzin V, Fernandez-Pozo N, Richter A, Schmelz EA, Schoettner M, Schafer M, Ahern KR, Meihls LN, Kaur H, Huffaker A, Mori N, Degenhardt J, Mueller LA, Jander G. 2015. Dynamic maize responses to aphid feeding are revealed by a time series of transcriptomic and metabolomic assays. Plant Physiology 169(3): 1727-1743.

Van Den Boom CEM, Van Beek TA, Posthumus MA, De Groot A, Dicke M. 2004. Qualitative and quantitative variation among volatile profiles induced by Tetranychus urticae feeding on plants from various families. Journal of chemical ecology 30(1): 69-89.

Verhage A, van Wees SCM, Pieterse CMJ. 2010. Plant Immunity: It's the hormones talking, but what do they say? Plant Physiology 154(2): 536-540.

Vranova E, Coman D, Gruissem W. 2013. Network analysis of the MVA and MEP pathways for isoprenoid synthesis. Annual Review of Plant Biology 64: 665-700.

Walling LL. 2008. Avoiding effective defenses: Strategies employed by phloem-feeding insects. Plant Physiology 146(3): 859-866.

War AR, Paulraj MG, Ahmad T, Buhroo AA, Hussain B, Ignacimuthu S, Sharma HC. 2012. Mechanisms of plant defense against insect herbivores. Plant Signaling and Behavior 7(10): 1306-1320.

Weirauch MT, Yang A, Albu M, Cote AG, Montenegro-Montero A, Drewe P, Najafabadi HS, Lambert SA, Mann I, Cook K, Zheng H, Goity A, van Bakel H, Lozano JC, Galli M, Lewsey MG, Huang EY, Mukherjee T, Chen XT, Reece-Hoyes JS, Govindarajan S, Shaulsky G, Walhout AJM, Bouget FY, Ratsch G, Larrondo LF, Ecker JR, Hughes TR. 2014. Determination and inference of eukaryotic transcription factor sequence specificity. Cell 158(6): 1431-1443.

Welter SC, Rosenheim JA, Johnson MW, Mau RFL, Gusukumaminuto LR. 1990. Effects of thrips-palmi and western flower thrips (Thysanoptera, Thripidae) on the yield, growth, and carbon allocation pattern in cucumbers. Journal of Economic Entomology 83(5): 20922101.

Windram O, Madhou P, McHattie S, Hill C, Hickman R, Cooke E, Jenkins DJ, Penfold CA, Baxter L, Breeze E, Kiddle SJ, Rhodes J, Atwell S, Kliebenstein DJ, Kim YS, Stegle O, Borgwardt K, Zhang CJ, Tabrett A, Legaie R, Moore J, Finkenstadt B, Wild DL, Mead A, Rand D, Beynon J, Ott S, Buchanan-Wollaston V, Denby KJ. 2012. Arabidopsis defense against Botrytis cinerea: Chronology and regulation deciphered by high-resolution temporal transcriptomic analysis. Plant Cell 24(9): 3530-3557.

Zhang PJ, Broekgaarden C, Zheng SJ, Snoeren TAL, van Loon JJA, Gols R, Dicke M. 2013. Jasmonate and ethylene signaling mediate whitefly-induced interference with indirect plant defense in Arabidopsis thaliana. New Phytologist 197(4): 1291-1299.

Zhu-Salzman K, Salzman RA, Ahn JE, Koiwa H. 2004. Transcriptional regulation of sorghum defense determinants against a phloem-feeding aphid. Plant Physiology 134(1): 420-431. 


\section{Supplementary figure}

A

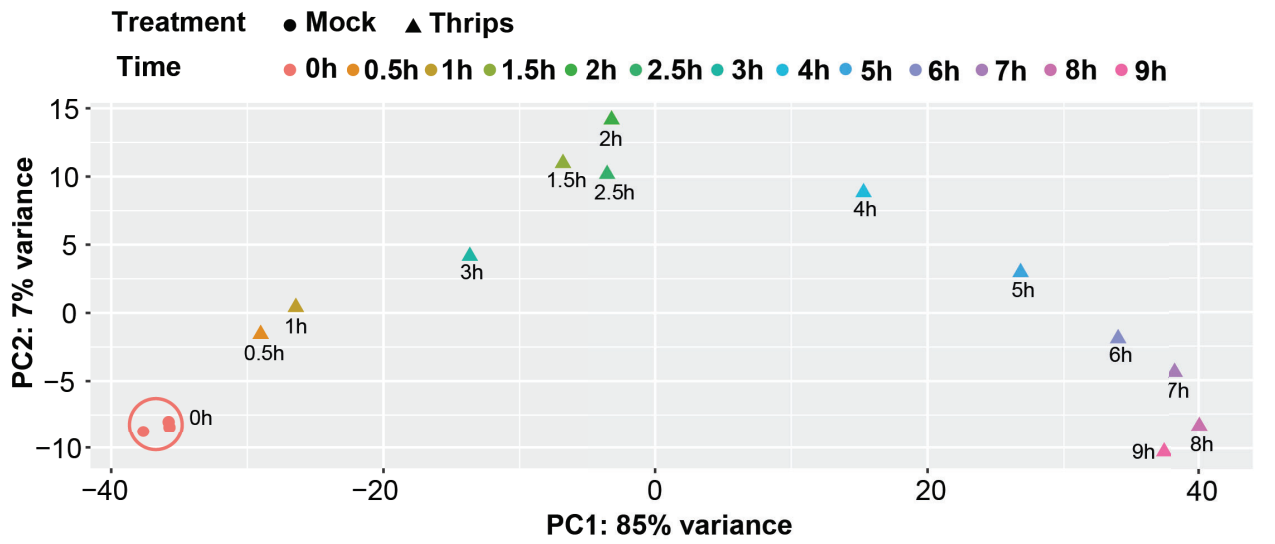

B

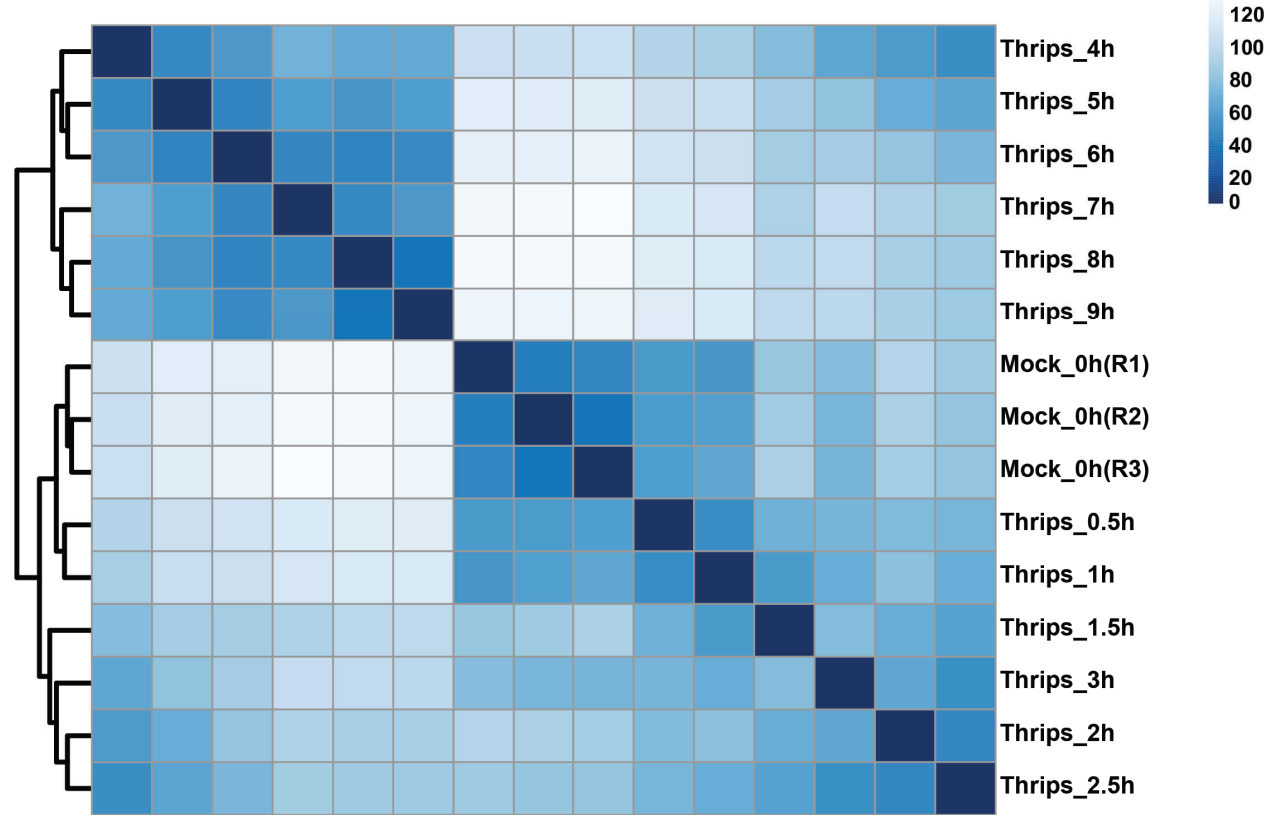

Figure S1. PCA and sample-to-sample distance analysis of transcriptome of sweet pepper infested (thrips) and non-infested (control/mock) plants. (A) PCA plot of sweet pepper non-infested (mock) and infested (thrips) transcriptome. PCA was generated on the regularized $\log _{2}$-transformed data with DESeq2 package in $\mathrm{R}$. Colours and shape indicate time points and treatments, respectively. Variation in percentage within the samples is depicted on both axes. (B) Sample-to-sample plot of sweet pepper non-infested (mock) and infested (thrips) transcriptome. It was generated on the regularized $\log _{2}$-transformed data with DESeq2 $R$ package. 




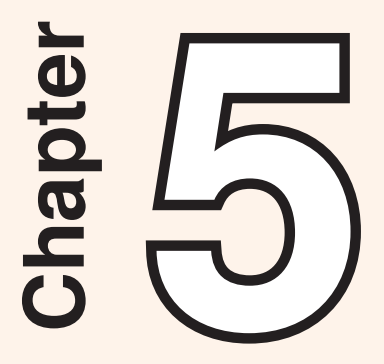

Comparative high-density transcriptomics reveals rapid and complex rearrangement of white cabbage transcriptome in response to thrips feeding

SJ. Sarde*, R. Hickman*, M. Steenbergen, G. Wiegers, CMJ. Pieterse, SCM. van Wees and M. Dicke

${ }^{*}$ These authors contributed equally to this work. 


\section{Abstract}

Temporal transcriptional rearrangements in response to insect feeding underlie a dynamically changing plant phenotype. Transcriptional rearrangements in response to the same herbivore in different plant species share commonalities but also have specific elements. To elucidate these, comparative transcriptomics using a high-density RNA-Seq approach provides a valuable tool. Here, we made a high-density ( 7 time points within $8 \mathrm{~h}$ ) gene expression analysis of white cabbage leaves in response to onion thrips (Thrips tabaci) feeding. One tenth (3790 up- and 2009 downregulated) of the white cabbage genome is differentially expressed within $8 \mathrm{~h}$ of onion thrips feeding. Cluster analysis identified 48 co-expressed gene clusters (32 up- and 16 downregulated) of which the up- and downregulated clusters are broadly associated with defence and development-related GO functional categories, respectively. Genes associated with phytohormones (JA, ET and SA) and secondary metabolites (phenylpropanoids, flavonoids, green-leaf volatiles and indolic glucosinolates) were rapidly induced, whereas the aliphatic glucosinolate pathway and development-related processes were suppressed. Comparative analyses between the onion-thrips induced transcriptome of white cabbage and the Western flower thrips (WFT)-induced transcriptome of Arabidopsis and sweet pepper revealed that the majority of the full-genome transcriptional responses against thrips are system-specific. More commonalities were found among upregulated genes than among downregulated genes. This suggests that the activation of biological processes is more similar among plants than the deactivation of biological processes. TF families like MYB, bHLH and WRKY were conserved in regulating responses to thrips across three plant species. A prominently conserved element among the three plant species is the JA biosynthesis and signalling pathway. The response of white cabbage to onion thrips is faster than the response of Arabidopsis and sweet pepper to WFT. This includes genes involved in the biosynthesis of phytohormones and secondary metabolites. This high-density comparative transcriptomic analysis provides insight into the complexity of the temporally dynamic response of plants to feeding by cell-content-feeding thrips.

Keywords: white cabbage (Brassica oleracea), onion thrips, time series, RNA-Seq, high-resolution, comparative transcriptomics, herbivory, defence 


\section{Introduction}

Plants face the attack by a wide range of herbivorous insects. The attack of a plant by an insect activates various mechanisms on a timescale of seconds to days. Upon herbivore perception, the earliest response is a change in plasma membrane potential $\left(\mathrm{V}_{\mathrm{m}}\right)$ involving fluctuations of cytosolic $\mathrm{Ca}^{2+}$ concentrations and followed by production of $\mathrm{H}_{2} \mathrm{O}_{2}$ (Maffei et al., 2007). Subsequently, kinases and phytohormones are induced. The phytohormones jasmonic acid (JA), ethylene (ET) and salicylic acid (SA) are central players in regulating defences against different herbivorous insects (Pieterse et al., 2009; Verhage et al., 2010; Pieterse et al., 2012; Stam et al., 2014). For example, the JA signalling pathway is induced by chewing insects like caterpillars (Reymond et al., 2004; De Vos et al., 2005) and cell-content-feeding insects like thrips (Abe et al., 2008; Abe et al., 2012; Sarde et al., 2018a; Steenbergen et al., 2018), whereas the SA signalling pathway is induced by phloem-feeding insects like aphids and whiteflies (Zhu-Salzman et al., 2004; Walling, 2008; Pieterse et al., 2012; Tzin et al., 2015; Broekgaarden et al., 2018). Moreover, ethylene (ET) often synergises with JA and fine-tunes JA-regulated defences against herbivorous insects (Pieterse et al., 2009; Pieterse et al., 2012; Stam et al., 2014). Phytohormones regulate the activation of gene transcription (time scale of minutes to hours) and the biosynthesis of metabolites (time scale of hours to days) (Maffei et al., 2007; Stam et al., 2014).

Thus, herbivore attack results in a dynamic reconfiguration of their transcriptome. Such transcriptional rearrangements result in a temporally dynamic reorganization of various biological processes (Windram et al., 2012; Lewis et al., 2015; Hickman et al., 2017; Sarde et al., 2019; Steenbergen et al., 2019). The temporal transcriptional rearrangements include the induction and repression of transcription factors (TFs) that regulate genes and processes involved in the biosynthesis of compounds such as phytohormones, primary and secondary metabolites, defence-related proteins or development-related pathways. This reorganization of transcriptional and biological processes dynamically influences the plant phenotype (Stam et al., 2014), subsequently altering interactions of the plant with plant-associated organisms. This can influence plants throughout the season (Poelman et al., 2010) or even over different seasons (Stam et al., 2018). Thus, early transcriptional responses of plants to attack by herbivorous insects can have extensive impact on plant ecology.

Studies of transcriptional responses to insect feeding usually include one or two time points over a period of 24 hours or longer, thus providing a low-resolution representation of the interaction (De Vos et al., 2005; Ehlting et al., 2008; Bidart-Bouzat \& Kliebenstein, 2011; Zhang et al., 2013; Appel et al., 2014; Diaz-Riquelme et al., 2016; Kroes et al., 2017; Broekgaarden et al., 2018; Tu et al., 2018). To gain comprehensive insight into how a plant temporally rearranges its transcriptome in response 
to attack by an insect herbivore, the collection of early high-density time-series transcriptional data is crucial. Such data are instrumental for unravelling the early phase of defence responses to insect herbivores, including the involvement of major TFs, phytohormones, sequential activation of biological processes and gene regulatory networks.

Furthermore, because high-density transcriptional analyses provide detailed information on early events in a plant's response, the data can be used to compare different insect-plant interactions for e.g. speed of response, chronology of gene transcriptional processes and biological processes activated, and complexity of temporal gene expression patterns. Recently, we made a high-resolution temporal assessment of Arabidopsis (Steenbergen et al., 2019) and sweet pepper (Capsicum annuum) (Sarde et al., 2019) transcriptomes in response to western flower thrips (WFT, Frankliniella occidentalis) feeding. This was done by detailed analysis of the transcriptome for 12 and 7 time points within the first 8 hours of attack on Arabidopsis and sweet pepper plants, respectively. This has yielded extensive information on similarities and differences in how these plants of different families respond to thrips infestation. In the present study, we made a high-resolution analysis of a third plant-thrips interaction, i.e., between white cabbage (Brassica oleracea) and onion thrips. One of the foci of this study is to analyse the expression pattern of white-cabbage genes involved in the biosynthesis of the defensive secondary metabolites of brassicaceous plants, such as glucosinolates and flavonoids (Schoonhoven et al., 2005).

Onion thrips, Thrips tabaci Lindeman (Thysanoptera: Thripidae), is a serious pest worldwide on various crops including White cabbage (Shelton et al., 2008; Fail et al., 2013). The pest status of onion thrips can be attributed to several characteristics, such as its short life-cycle, high reproductive rate, polyphagous nature, thigmokinetic behaviour and rapid development of resistance to insecticides (Diaz-Montano et al., 2011; Gill et al., 2015; Steenbergen et al., 2018). These insects cause direct damage to plants by rupturing the epidermal and mesophyll cells and ingesting the cell contents. Moreover, they also cause indirect damage on plants by transmitting tospoviruses like Iris yellow spot virus (IYSV) (Bunyaviridae) (Diaz-Montano et al., 2011; Gill et al., 2015).

In contrast to WFT, molecular responses of plants to onion thrips have not received much attention. The main goals of the present study were: 1) to comprehensively investigate the temporal transcriptomic response of white cabbage to feeding by onion thrips through high-resolution transcriptomics, and 2) to compare the response of white cabbage plants to onion thrips to the transcriptomic response of Arabidopsis and sweet pepper plants to WFT through comparative transcriptomics to extend our knowledge of thrips-induced transcriptional plant responses. 


\section{Materials and methods}

\section{Plant material and onion thrips}

White cabbage [Brassica oleracea (W0246 variety, Syngenta, Enkhuizen, The Netherlands)] plants were grown in a greenhouse at $22 \pm 5^{\circ} \mathrm{C}$ (day/night), $16 \mathrm{~L}: 8 \mathrm{D}$ photoperiod, $60 \pm 10 \%$ relative humidity and $130 \mu \mathrm{mol}$ photons $\mathrm{m}^{-2} \mathrm{~s}^{-1}$ of light intensity. For thrips infestation, four-week-old white cabbage plants were used. Onion thrips (Thrips tabaci) were reared in jars (10 cm diameter) on leek leaves (Allium ampeloprasum) in a climate-controlled room $\left(25 \pm 2^{\circ} \mathrm{C}, \mathrm{L} 16: 8 \mathrm{D}\right.$ photoperiod and $70 \pm 10 \%$ relative humidity).

\section{Thrips treatment, RNA extraction and library preparation}

Five $2^{\text {nd }}$ instar larvae (L2) of onion thrips, confined in clip cages ( $3 \mathrm{~cm}$ diameter), were used to infest the second true leaf of four-week-old white cabbage plants. One clip cage was used per plant. Empty clip cages without thrips served as mock treatment. For each time point and treatment, the leaf area underneath the clip cages was harvested using a cork borer ( $3 \mathrm{~cm}$ diameter), flash frozen in liquid nitrogen and stored at $-80{ }^{\circ} \mathrm{C}$. Time points of harvesting were $0.5,1,1.5,2,2.5,3,4,5,6,7,8$, and $9 \mathrm{~h}$ after the introduction of the five thrips larvae. Three biological replicates were collected for each time point and treatment. Each individual biological replicate represents one single white cabbage plant. Extraction of RNA was executed using the RNeasy Plant Mini Kit (QIAGEN), according to the manufacturer's protocol. DNAase I treatment on column was conducted for all the samples during RNA extraction. RNA quantity and quality were assessed by Nanodrop and Agilent 2100 bioanalyzer, respectively. Samples with RNA Integrity Number $(\mathrm{RIN}) \geq 7$ were used for RNA library preparation. Samples were prepared according to the TruSeq Stranded mRNA HT Sample Prep Kit from Illumina (Illumina Inc., San Diego, CA, USA). This protocol identifies strand-specific transcripts. Sequencing of samples was performed with an Illumina Hi-seq 2000 platform. Samples were randomly allocated to seven lanes (Illumina flow cells) within each run.

\section{Quality control, alignment and differential gene expression of RNA-Seq dataset}

Quality of raw RNA-Seq reads was assessed using the FastQC (https://www.bioinformatics.babraham.ac.uk/projects/fastqc/) tool. Trimmomatic was used to trim the initial 14-basepairs (bp) and Illumina adapters. Reads below 25 bp length were excluded from analysis for all samples (Bolger et al., 2014).

Alignment of RNA-Seq reads was performed using TopHat2 (v2.0.14) (Kim et al., 2013) using the following parameters: 'p 4', '--bowtie-n', '--min-intron-length 40', '--max-intron-length 2000', '-N 4', '--no-novel-juncs', '--read-gap-length 2', '--read-edit- 
dist 4'. The aligned reads to each B. oleracea (v2.1) (Parkin et al., 2014) gene model were summarized using HTSeq-count (v.0.9.1) (Anders et al., 2015) with parameters: '--stranded no', '-i ID', '-t mRNA'. Principal Component Analysis (PCA) and sample-to-sample distance plots were generated in R (https://www.r-project.org/) using regularized $\log _{2}$-transformed data using the DESeq2 package (Love et al., 2014; Love et al., 2015).

For analysis of differentially expressed genes (DEG), the DESeq2 R Bioconductor package (Love et al., 2014; Love et al., 2015) in R was used. Prior to DEG analysis, raw read counts were normalized for sequencing depths across all samples using the DESeq2's count normalization procedure. A negative binomial likelihood ratio test (nbinomLRT) was used to identify DEGs between mock and thrips-infested plants. For this analysis, both treatment and time post-treatment were considered as factors. Genes with $\log _{2}$-fold change $\geq 0.5$ or $\leq-0.5$ at one or more time points, a Bonferroni-corrected $P$ value $<0.01$ and read counts $\geq 20$ at least in one sample were qualified as DEGs.

\section{Gene clustering, TF family and promoter motif enrichment analyses}

SplineCluster, a time series-clustering algorithm, was used on $\log _{2}-F C$ profiles of DEGs at each time point, to partition the DEGs into clusters based on temporal expression profiles (Heard et al., 2006). SplineCluster was used with the following parameters: a prior precision stringency of $10^{-4}$, the default normalization procedure and cluster reallocation step (Heard, 2011). Default values were used for all other optional parameters.

For TF family abundance analysis, we investigated overrepresentation of TF families within DEGs induced upon thrips feeding in white cabbage. Before this analysis, the TF families in white cabbage were determined using 4272 TFs of $B$. oleracea from the Plant Transcription Factor Database (http://planttfdb.cbi.pku.edu.cn/index.php) (Jin et al., 2017) using blastp with stringency E-value $<10^{-4}$. The hypergeometric distribution was used to identify overrepresentation of TF families within sets of DEGs. $P$ values were corrected for multiple testing with the Bonferroni method.

To identify TF binding motifs in promoters of temporally clustered DEGs, comparison was made to 580 characterized Arabidopsis TF DNA-binding motifs from Franco-Zorrilla et al. (2014) and CIS-DB (version 1.02) (Weirauch et al., 2014). FIMO (Grant et al., 2011) was used to determine the occurrence of particular motifs within the promoter sequences (500 bp upstream to the start codon) of all white cabbage genes. A motif was considered to be present in a promoter sequence, if it had at least one match with a $P$ value $<10^{-4}$. The hypergeometric distribution against the background of all white cabbage genes was used to assess motif enrichment in a given cluster. 


\section{Identification of chronology of defence pathways upon thrips feeding}

Pairwise comparison between mock and thrips-treated samples at each time point was performed using DESeq2 package (Love et al., 2014; Love et al., 2015) in R. Genes with $\log _{2}$-fold change $\geq 0.5$ or $\leq-0.5$ and a Bonferroni-corrected $P$ value $<$ 0.01 were considered to be differentially expressed. For the small number of genes that did not meet these criteria, the time point of first differential expression was defined by minimal $P$ value. The output files were further processed to identify and categorize DEGs into two categories: first time of differential expression (ftode) and again $\underline{\text { differential }}$ expression (ade).

\section{Gene Ontology (GO)-term enrichment analysis}

For GO-term enrichment analysis, Cytoscape (Shannon et al., 2003) was used for Arabidopsis and GOAtools (v0.7.9) (Klopfenstein et al., 2018), a python-based library, was used for white-cabbage and sweet pepper, using Fisher's exact test. For this, we used the GO-annotated proteome for $B$. oleracea from the Plaza database (https://bioinformatics.psb.ugent.be/plaza/) (Jin et al., 2017). Overrepresentation of GO categories like "biological process", "cellular component" and "molecular function" was analyzed at $P<0.05$.

\section{Comparative transcriptomics}

Comparative transcriptomics was performed between B. oleracea and Arabidopsis (TAIR10) and between B. oleracea and Capsicum annuum L. Zunla proteome using the Bi-directional Best Hit (BBH) method (Martel et al., 2015). Local blastp with stringency E-value $<10^{-4}$ was used to identify one-to-one orthologues in both comparisons. The Arabidopsis and pepper transcriptome datasets used in this study relate to plants induced by Frankliniella occidentalis (Western flower thrips) according to methodology very similar to that used in the present study (Sarde et al., 2019; Steenbergen et al., 2019).

\section{Results}

Temporal transcriptomic response of white cabbage plants upon onion thrips feeding

To gain detailed insight into the temporally dynamic transcriptomic response of white cabbage plants upon feeding by onion thrips, individual samples from 12 time points $(0.5,1.0,1.5,2.0,2.5,3.0,4.0,5.0,6.0,7.0,8.0$, and $9.0 \mathrm{~h}$ of infestation) plus 3 samples of the $0 \mathrm{~h}$ time point were sequenced and analysed. The PCA and heat map of sample-to-sample distance analysis show a gradual development of the transcriptom- 
ic response with time in the white cabbage plants (Fig. S1). This analysis led to the selection of the 1, 2, 3, 4, 6 and $8 \mathrm{~h}$ time points for sequencing of additional samples and subsequent in-depth analysis. Thus, including the $0 \mathrm{~h}$ time point, the time series consists of seven time points. The detailed data for these seven time points were subjected to PCA to investigate the effect of treatment and time on the white cabbage transcriptome in response to onion thrips feeding. PCA explicitly discriminates the samples for treatments and time points, indicating that both treatment and time since infestation are determinants of the transcriptomic response of white cabbage (Fig. 1A). The mock-treated samples differ between time points, suggesting a diurnal-rhythm effect on the transcriptome. The three replicates per treatment and time point exhibit considerable similarity apart from the first time point for thrips-infested plants (1h).

A

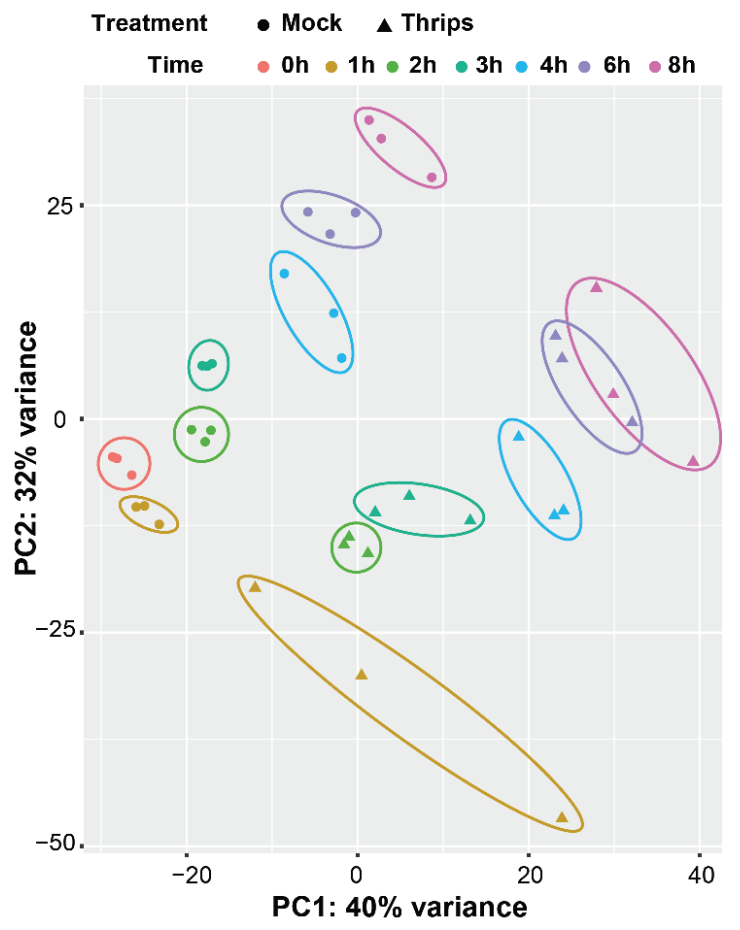

B
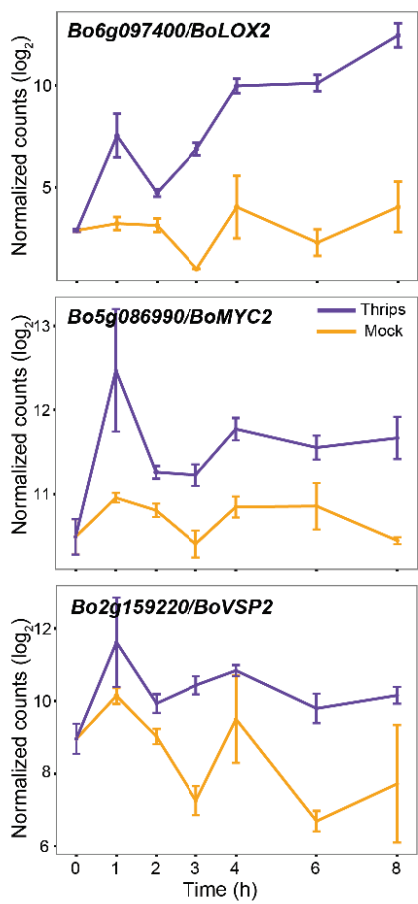

Figure 1. Principal component analysis (PCA) of white-cabbage transcriptome of mock-treated and onion thrips-infested plants at several time points and expression patterns of selected JA-associated marker genes of white cabbage. (A) PCA plot of white-cabbage whole-genome transcriptomic response at seven time points for mock-treated and thrips-infested plants. Variation between the samples of treatment and time post-treatment is depicted on both axes. Different shapes represent the different treatments and the different colours represent the different time points. (B) Temporal expression profile of JA-related marker genes from transcriptome dataset. Expression of each gene depicts mean $\pm \mathrm{SE}$ of 3 biological replicates. 
Next, we used the DESeq2 negative binomial log-ratio test with treatment and time since start of treatment as factors, to identify 5799 differentially expressed genes (DEGs) between mock (Supplemental Data Set 1). This accounts for $9.7 \%$ of the total 59,225 white cabbage genes (Parkin et al., 2014). Because thrips are known to induce the JA pathway (De Vos et al., 2005; Abe et al., 2008; Sarde et al., 2018a; Sarde et al., 2018b), the expression profiles of several genes in the JA pathway (BoLOX2, BoMYC2, BoVSP2) were specifically analysed. Reassuringly, all three genes were rapidly up-regulated by thrips feeding (Fig. 1B).

\section{Gene clusters, TF family abundance and TF motif analysis}

To identify the predominant dynamic patterns of gene expression in plants during thrips feeding, we used the time-series-clustering algorithm, SplineCluster (Heard et al., 2006), to cluster the 5799 DEGs based on their expression pattern over time. The SplineCluster analysis identified 48 coexpressed gene clusters: 16 down-and 32 upregulated clusters. The 16 downregulated (clusters 1-16) and 32 upregulated (clusters 17-48) gene clusters represent 2009 (34.6\%) and 3790 (65.4\%) DEGs, respectively (Fig. 2A) (Supplemental Data Set 2). Among the upregulated clusters, several (clusters 20-29, 35 and 41-48) showed that gene expression is rapidly induced within $1 \mathrm{~h}$ of thrips feeding, whereas in other clusters (cluster 30-34 and 36-40) genes were initially downregulated, followed by subsequent upregulation. Similarly, in the clusters representing downregulated genes, rapid downregulation is seen in some clusters (4-10), while genes in other clusters (1, 2 and 11-16) show a gradual downregulation over time (Fig. 2A). To explore the biological processes associated with the dynamic expression patterns identified by the SplineCluster analysis, GOATOOLS (Klopfenstein et al., 2018) was used to identify significantly overrepresented functional categories associated with genes in each cluster. Several upregulated clusters (clusters 42-45 and 48) are enriched with genes associated with GO terms like "Jasmonic acid signalling pathway" and "Response to JA" (Fig 2A). Other defence-related pathways are overrepresented in other clusters. For example, cluster 27 with "Tryptophan biosynthetic process", cluster 36 with "Anthocyanin-containing compound biosynthesis", cluster 35 with "Defence response to fungus" (Supplemental Data Set 3). Similarly, downregulated clusters (clusters 1-16) exhibit GO terms especially associated with growth and development (Supplemental Data Set 4). For example, clusters 2 and 3 with "Photosynthetic electron transport in photosystem I", cluster 4 with "Photosynthesis, light harvesting", clusters 12, 13 and 14 with "Protein phosphorylation" and cluster 16 with "Protein ubiquitination". Cluster 12 is overrepresented with genes involved in "Terpenoid biosynthesis". 
A

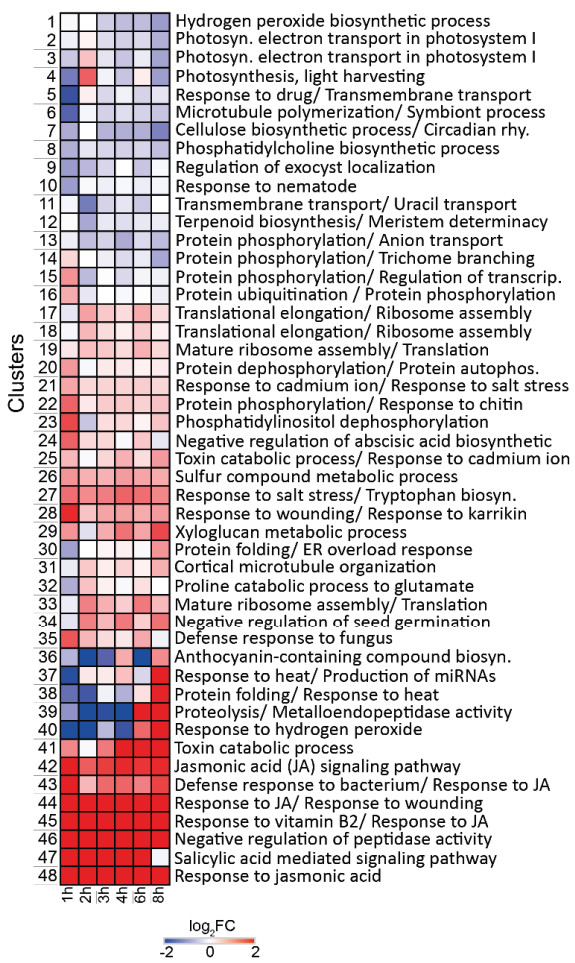

B

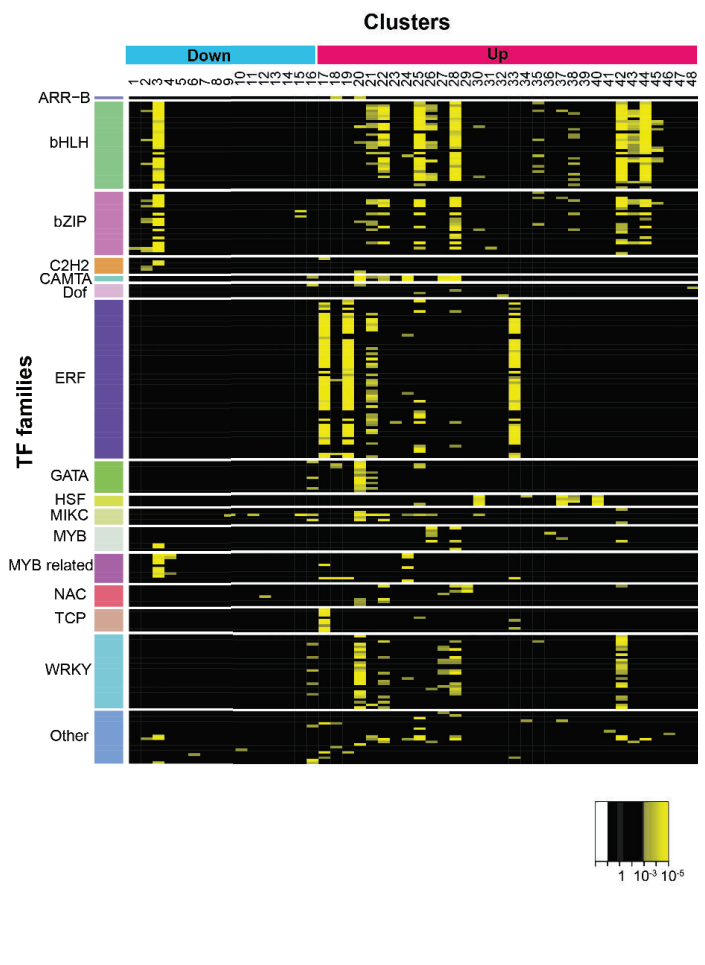

Figure 2. Temporally co-expressed gene clusters and promoter motif analysis of gene clusters. (A) Heat map showing mean expression profiles of 48 gene clusters (clusters 1-16 downregulated and clusters 17-48 upregulated) with selected GO terms. (B) Overrepresented TF binding motifs in the 48 gene clusters. Enriched TF family binding motifs in each cluster are shown in yellow colour.

To investigate which TF families are involved in regulating the white cabbage response to onion thrips feeding, TF abundance was analysed for all up- (3790) and downregulated (2009) genes. We identified 2910 non-redundant TFs in the $B$. oleracea (TO1434) proteome (Parkin et al., 2014) using 4272 TFs of Brassica oleracea from the Plant Transcription Factor Database (Supplemental Data Set 5). In the 3790 upregulated genes, several TF families like ERF, WRKY, MYB, HSF, GRAS, bHLH, MYB-related, RAV and Trihelix are overrepresented. Similarly, in the 2009 downregulated genes, the TF families ARF, NF-YA, YABBY, C2H2, MYB_related, CAMTA, AP2, G2-like, Nin-like, HD-ZIP, bHLH, TALE and Dof are significantly overrepresented (Supplemental Data Set 6).

Subsequently, we searched for TF binding motifs in the promoter sequences of the genes in all 48 clusters. For this, we used FIMO (Grant et al., 2011) and 580 characterized Arabidopsis TF DNA-binding motifs from CIS-DB (version 1.02) (Franco-Zorrilla 
et al., 2014; Weirauch et al., 2014). In clusters of upregulated genes (clusters 17-48), motifs that correspond to bHLH, bZIP, ERF and WRKY TFs are overrepresented (Fig. $2 \mathrm{~B})$, suggesting expression of genes in these clusters is regulated by members of these TF families. The motif enrichment also matches the coordinated up-regulation of genes encoding members of these TF families following thrips feeding.. Clusters 22, 25, 28, 42 and 44 are enriched with binding sites of bHLH and bZIP TFs, clusters 17,19 and 33 with binding sites of ERF TFs and clusters 20, 28 and 42 with binding sites of WRKY TFs. In clusters with downregulated genes (clusters 1- 16), binding sites for bHLH, bZIP, MYB-related and MIKC TFs are overrepresented (Fig. 2B).

\section{Chronology of phytohormone induction}

The phytohormones JA, ET and SA regulate induced defences against insect herbivores. To investigate how these phytohormonal pathways are involved in the response of white cabbage plants to onion thrips, we examined the temporal expression dynamics of genes involved in these pathways. Several of the JA-biosynthetic (BoLOX2, BoAOS, BoAOC3, BoOPR3, BoACX1) and JA-signalling (BoJAZ1-3, BoJAZ5-9) genes are significantly upregulated within $1 \mathrm{~h}$ of thrips feeding (Fig. 3A). Downstream JA-responsive genes like BoMYC2 and BoVSP2 are significantly induced at $2 \mathrm{~h}$ and $3 \mathrm{~h}$ since the start of thrips feeding, respectively. Similarly, many ET-related genes are induced within 1-2 $\mathrm{h}$ of thrips feeding (Fig. 3B). SA biosynthesis may occur via the phenylalanine (PAL) or isochorismate pathway (ICS). Yet, the ICS pathway is crucial for the production of SA that is involved in plant defence against pathogens (Wildermuth et al., 2001). We analysed the transcriptional responses of PAL and ICS genes. Several homologs of $P A L$ genes are upregulated within $2 \mathrm{~h}$ of thrips feeding. In contrast, BolCS is downregulated at the $3 \mathrm{~h}$ time point. The downstream SA-responsive gene, BoPR5 (Ali et al., 2017), is significantly induced for the first time at the $6 \mathrm{~h}$ time point. This indicates downregulation of SA via the ICS pathway, upregulation of the phenylalanine (PAL) pathway and late upregulation of SA-responsive genes (Fig. $3 \mathrm{C})$. In conclusion, this analysis suggests an induction of all three major defence-related hormones in white cabbage upon thrips feeding, albeit at different time scales.

\section{Induction of secondary metabolites}

To evaluate if feeding by onion thrips induces secondary metabolites such as phenylpropanoids, flavonoids, green leaf volatiles (GLVs) and glucosinolates (GLS), the temporal expression pattern of genes involved in these pathways was analysed. The majority of phenylpropanoid and flavonoid biosynthetic genes (BoPAL, BoC4H, Bo$4 C L, B o F 3 H, B o F L S$ ) (Onkokesung et al., 2014) show significant upregulation at the $2 \mathrm{~h}$ time point, with the exception of $\mathrm{BoCHS}, \mathrm{BoCH}$ and a few homologs of Bo4CL, which are downregulated at this time point (Fig. 4A). 
A

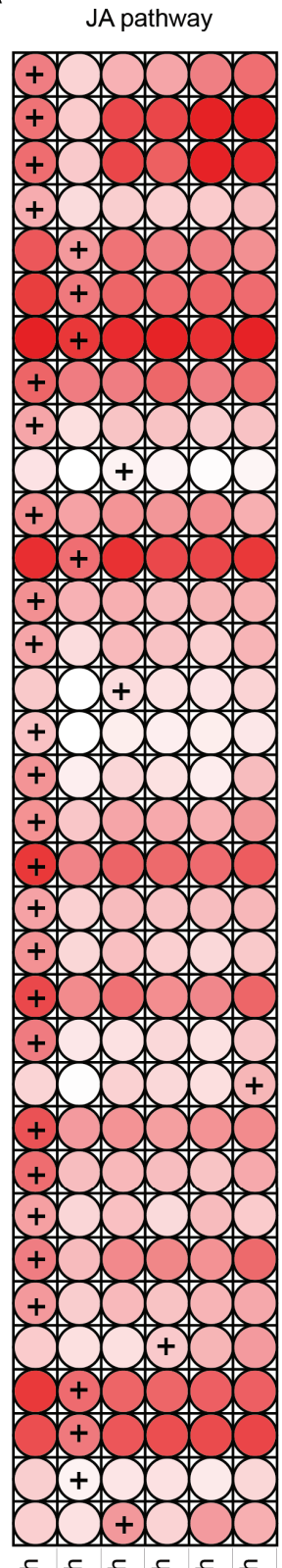

Bo2g050630/BoLOX2 Bo6g097400/BoLOX2 Bo01163s010/BoLOX2 Bo2g116210/BoAOS Bo7g082010/BoAOC2 Bo7g082020/BoAOC2 Bo9g075840/BoAOC2 Bo9g075870/BoAOC3 Bo3g086880/BoOPR3 Bo1g053250/BoACX1 Bo5g027170/BoJAZ1 Bo8g102890/BoJAZ1 Bo8g068340/BoJAZ1 Bo2g082410/BoJAZ2 Bo6g086720/BoJAZ2 Bo6g119140/BoJAZ2 Bo5g121480/BoJAZ3 Bo8g104890/BoJAZ5 Bo8g067140/BoJAZ5 Bo2g076750/BoJAZ6 Bo6g115920/BoJAZ6 Bo3g027780/BoJAZ7 Bo4g182750/BoJAZ7 B04g039500/BoJAZ7 Bo3g147640/BoJAZ8 Bo5g062450/BoJAZ8 Bo2g068460/BoJAZ9 Bo6g094620/BoJAZ9 Bo6g112300/BoJAZ9 Bo2g011150/BoJAZ10 Bo3g009050/BoJAZ10 Bo9g168900/BoJAZ10 Bo5g086990/MYC2 Bo2g159220/BoVSP2

ᄃ
B

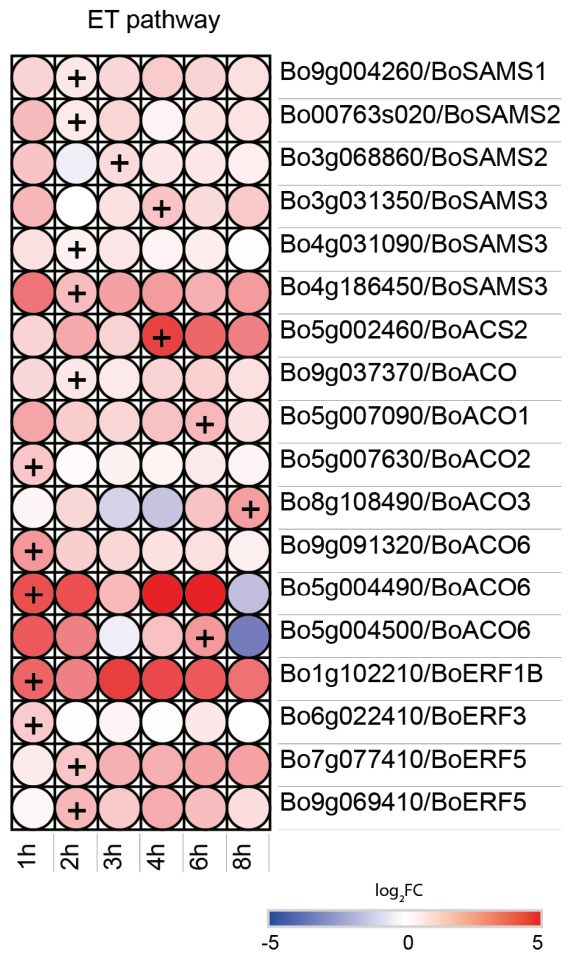

C SA pathway

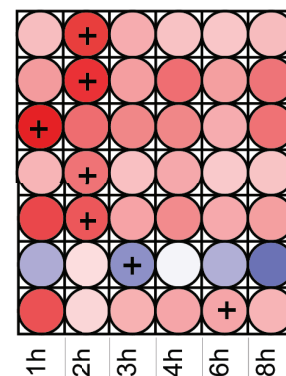

Bo4g030910/BoPAL Bo4g186590/BoPAL Bo6g067250/BoPAL Bo8g082620/BoPAL Bo00703s080/BoPAL Bo6g086860/BolCS Bo2g159270/BoPR5

$=\bar{N} \bar{m} \frac{\bar{c}}{0}$

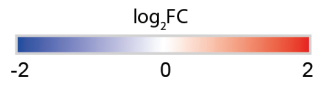

Figure 3. Temporal expression of genes involved in JA, ET and SA hormonal pathways differentially expressed upon onion thrips feeding. (A) JA pathway, (B) ET pathway and (C) SA pathway. Expression of genes represents $\log _{2}$ fold change between mock- and thrips-infested plants for each time point. ' + ' indicates significant $(P<0.01)$ first time of differential expression (ftode) for each gene. 
Glucosinolates (GLS), are broadly divided into three groups: aliphatic, aromatic and indolic glucosinolates (Halkier \& Gershenzon, 2006; Ishida et al., 2014; Frerigmann et al., 2016). Among the DEGs in our dataset, we found major regulators of indolic glucosinolates (BoMYB34, BoMYB122, BoMYB51) (Frerigmann et al., 2016) and several downstream genes (BoASA1, BoTSA1, BoCYP83B1, BoGSTF9, BoGSTF10, BoSUR1, BoUGT74B1, BoSOT16) (Tytgat et al., 2013) to be induced within 1-2 $\mathrm{h}$ of thrips feeding (Fig. 4B). In contrast, the major upstream regulator of aliphatic GLS (BoMYB28) (Halkier \& Gershenzon, 2006) was suppressed within $2 \mathrm{~h}$ of thrips feeding (Fig. 4C). In addition, the expression of various other major genes involved in the biosynthesis of aliphatic GLS, such as BoMYB29, BoMYB76, BoBAT5 and BoMAM1-3 (Tytgat et al., 2013) did not alter upon thrips feeding. This suggests that specific induction of indolic GLS and suppression of aliphatic GLS occurs upon onion thrips feeding. Both homologues of the hydroperoxide lyase (HPL) gene, known to break down lipid hydroperoxides to produce green leaf volatiles (Bate et al., 1998), are upregulated within $1 \mathrm{~h}$ of thrips feeding (Fig. 4D).

\section{Chronology of biological processes altered in response to thrips feeding}

To gain in-depth understanding of how white cabbage plants reconfigure their transcriptome over time and sequentially activate different biological processes, we performed a pairwise comparison for all individual time points between mock treatment and thrips treatment. This analysis showed progressive transcriptional reconfiguration over time and partitioned both up- and downregulated DEGs into the classes first time of $\underline{\text { differential }}$ expression (ftode) and again differentially expressed (ade) (Supplemental Data Set 7). The major transcriptional burst in both up- and downregulated genes, occurred rapidly, i.e. within 1-2 $\mathrm{h}$ of thrips feeding (Fig. 5). The majority of DEGs in this study are differentially expressed within $2 \mathrm{~h}$ since the start of thrips feeding. Approximately $650-800$ of the upregulated genes at 3-8 h were also upregulated at earlier time points. In contrast, only few downregulated genes at 3-8 h since the start of thrips feeding are again upregulated, suggesting that the upregulation of genes is more pronounced than the downregulation. Thus, this analysis illustrates temporal reconfiguration of the white-cabbage transcriptome and its initial transcriptional burst in response to thrips feeding occurring within 1-2 $\mathrm{h}$ of thrips feeding. 
A

Phenylpropanoids and flavanoids

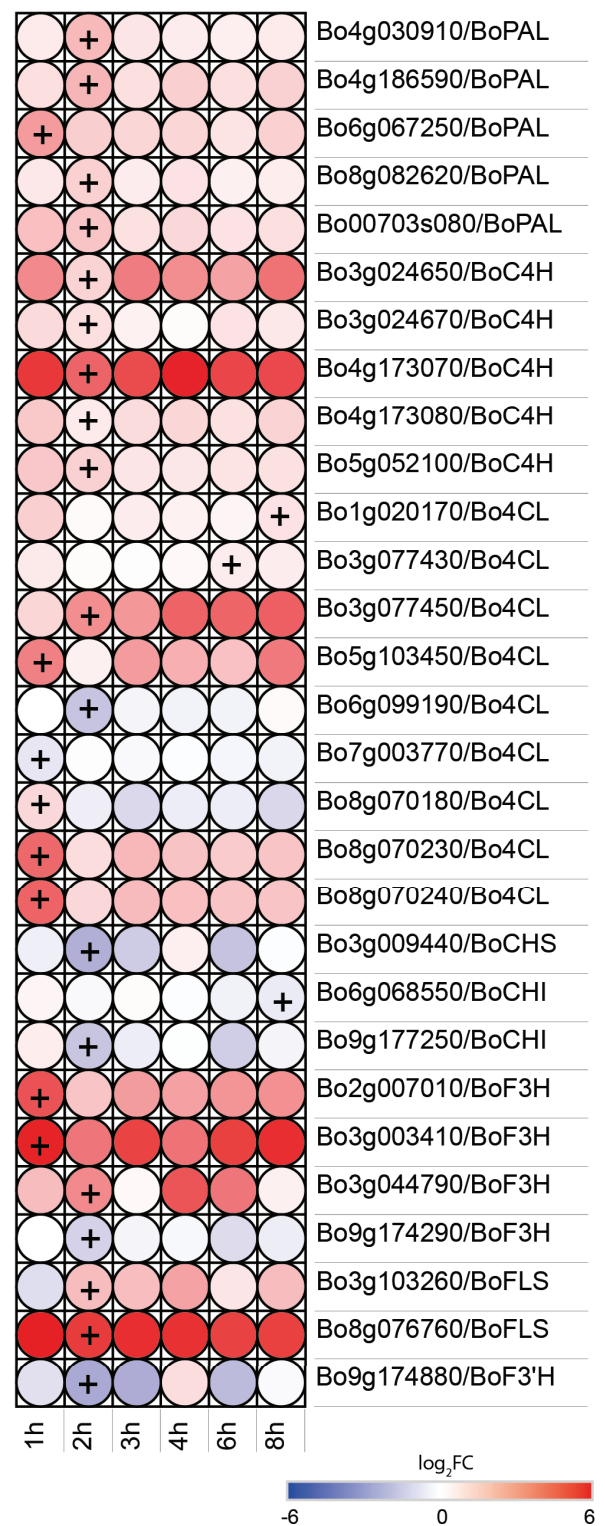

B

Indole glucosinolates

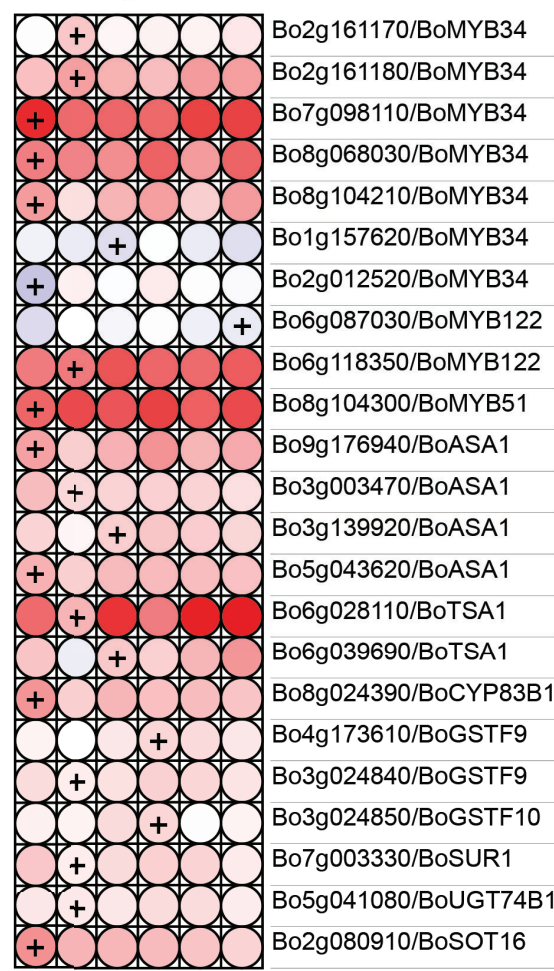

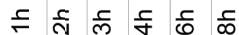

$\log _{2} \mathrm{FC}$

C

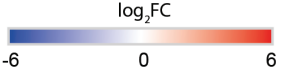

Aliphatic glucosinolates

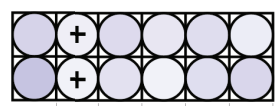

Bo9g014610/BoMYB28 Bo2g161590/BoMYB28

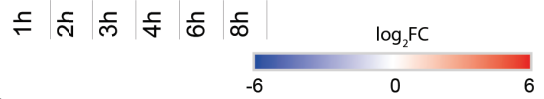

D

Green leat volatiles (GLVs)

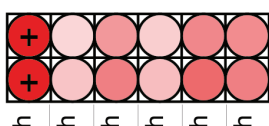

Bo7g103730/HPL Bo7g103740/HPL

다 다

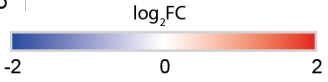

Figure 4. Temporal expression of genes involved in the biosynthesis of secondary metabolites . (A) phenylpropanoid and flavonoid pathways, (B) Indole glucosinolates (GLS), (C) Aliphatic glucosinolates (GLS), and (D) green-leaf volatiles. Expression of genes represents $\log _{2}$ fold change between mock- and thrips-infested plants for each time point. ' + ' indicates significant $(P<0.01)$ first time of differential expression (ftode) for each gene. 
To analyse the chronology of biological processes activated upon thrips feeding, we investigated the GO terms associated with genes showing first time of differential expression (ftode) at each time point. At $1 \mathrm{~h}$ after the start of thrips feeding, the GO terms overrepresented among upregulated genes are mainly associated with functional categories like "Jasmonic acid mediated signalling pathway" and "Response to ethylene", indicating rapid induction of these phytohormonal pathways (Fig. 5). At the $2 \mathrm{~h}$ time point, GO terms like "Regulation of systemic acquired resistance", "Aromatic amino acid family biosynthetic process" are overrepresented, reflecting induction of resistance and biosynthesis of aromatic amino acids (Phe, Tyr, Trp), which act as precursors for several secondary metabolites in plants (Tzin \& Galili, 2010). Among the downregulated genes, GO terms associated with plant development like "Response to auxin", "Photosynthesis", and "Photosynthetic electron transport in photosystem-l" are overrepresented.

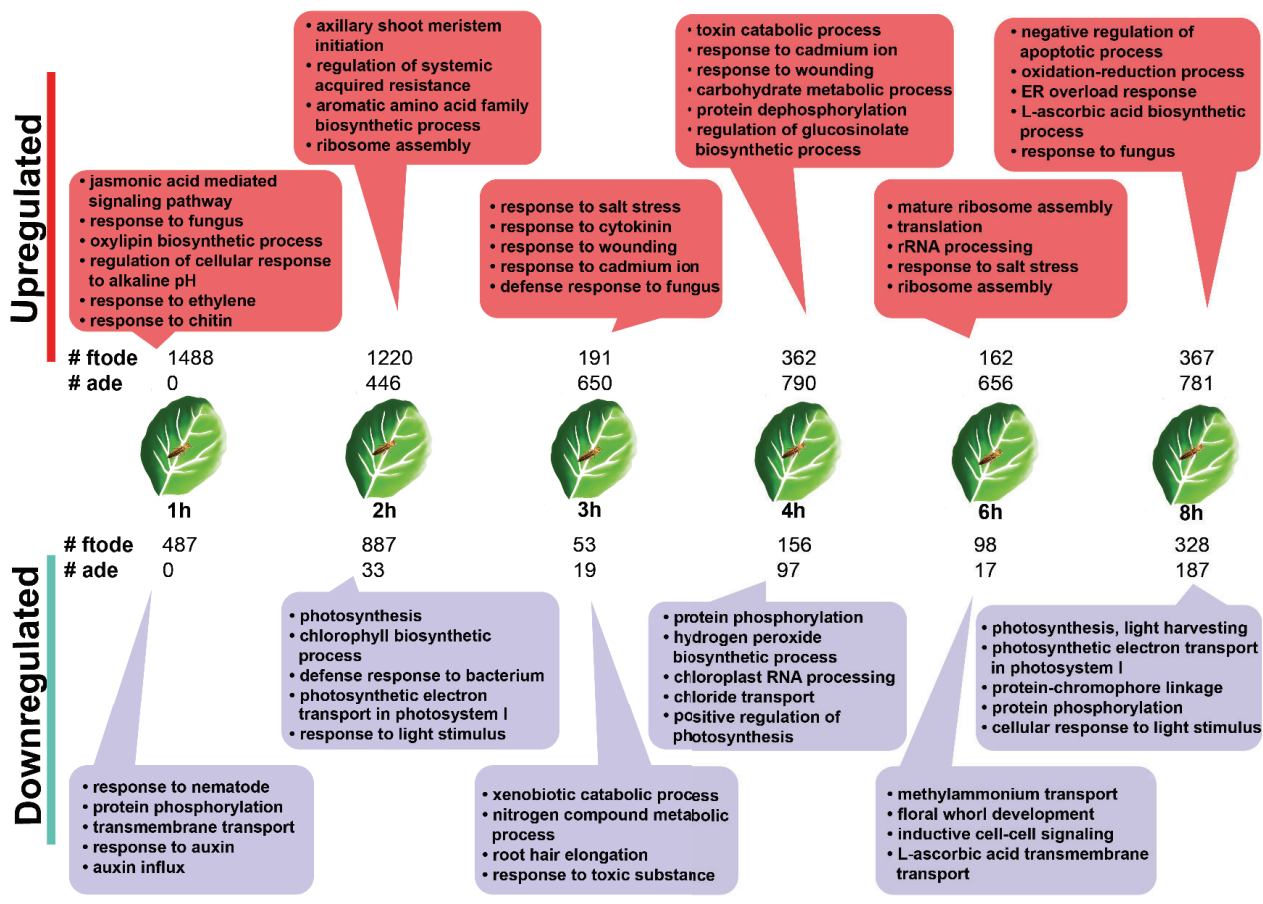

Figure 5. Chronology of white cabbage biological processes reorganized in response to onion thrips feeding. Number of first time differentially expressed (ftode) and again differentially expressed (ade) genes for both up- and downregulated genes are depicted above and below pictures of cabbage leaves, respectively. Light green and light blue boxes show selected GO terms for ftode up- and downregulated genes, respectively. 


\section{Comparative transcriptomics: white cabbage vs Arabidopsis and white cab- bage vs sweet pepper}

Recently, similar high-density time series data were generated for Arabidopsis (Steenbergen et al., 2019) and sweet pepper (Capsicum annuum) (Sarde et al., 2019) plants in response to WFT feeding. These studies identified a total of 2788 (1820 up- and 968 downregulated) DEGs in Arabidopsis and 3062 (2060 up- and 1002 downregulated) DEGs in sweet pepper over a span of 8 hours of thrips feeding. To gain insight into the commonalities and specifics of whole-genome transcriptional responses on plant-family and plant-species level upon feeding by different thrips species, we compared onion thrips induced white-cabbage DEGs $(5799 ; 3790$ upand 2009 downregulated) with WFT-induced Arabidopsis (Steenbergen et al., 2019) and sweet pepper DEGs (Sarde et al., 2019).

In the comparison of DEGs between white cabbage and Arabidopsis, 1938 of 3790 upregulated genes and 1093 of 2009 downregulated genes from white cabbage have Arabidopsis orthologues. Likewise, 1420 of 1820 upregulated genes and 708 of 968 downregulated genes from Arabidopsis have white cabbage orthologues (Fig. 6). In the comparison of white cabbage vs sweet pepper, we identified that 1126 of 3790 upregulated genes and 730 of 2009 downregulated genes of white cabbage possess sweet pepper orthologues. Likewise, 986 of 2060 upregulated genes and 468 of 1002 downregulated genes of sweet pepper possess white cabbage orthologues (Fig. 7). The relatively high number of orthologues between white cabbage and Arabidopsis compared to the comparison of white cabbage and sweet pepper, reflects their shared brassicaceous identity. Furthermore, in both comparisons, large numbersof genes [in white cabbage vs Arabidopsis, 1225 up- and 970 downregulated in white cabbage and 754 up- and 538 downregulated in Arabidopsis; in white cabbage vs sweet pepper, 779 up- and 602 downregulated in white cabbage and 624 up- and 355 downregulated in sweet pepper] have orthologues in the other plant species, but those orthologues are not differentially expressed. Thus, a large proportion of DEGs show differential expression in a species-specific manner (Fig. 6 and 7). This indicates that the majority of the transcriptomic responses in each plant species is distinct. It is interesting to note that more commonalities exist in the subset of upregulated genes than in the subset of downregulated genes: 622 of the 1938 (32\%) upregulated white cabbage genes that have Arabidopsis orthologues are also upregulated in Arabidopsis, compared to 79 out of 1093 (7\%) downregulated white cabbage genes with orthologous Arabidopsis genes that are downregulated in both species. Moreover, this is similar for the white cabbage vs sweet pepper comparison: 315 upregulated genes out of $1126(27 \%)$ differentially regulated sweet pepper genes that are upregulated in sweet pepper and cabbage versus 81 out of $730(11 \%)$ downregulated genes. This suggests that the activation of biological processes is more similar among plants than 
the deactivation of biological processes.

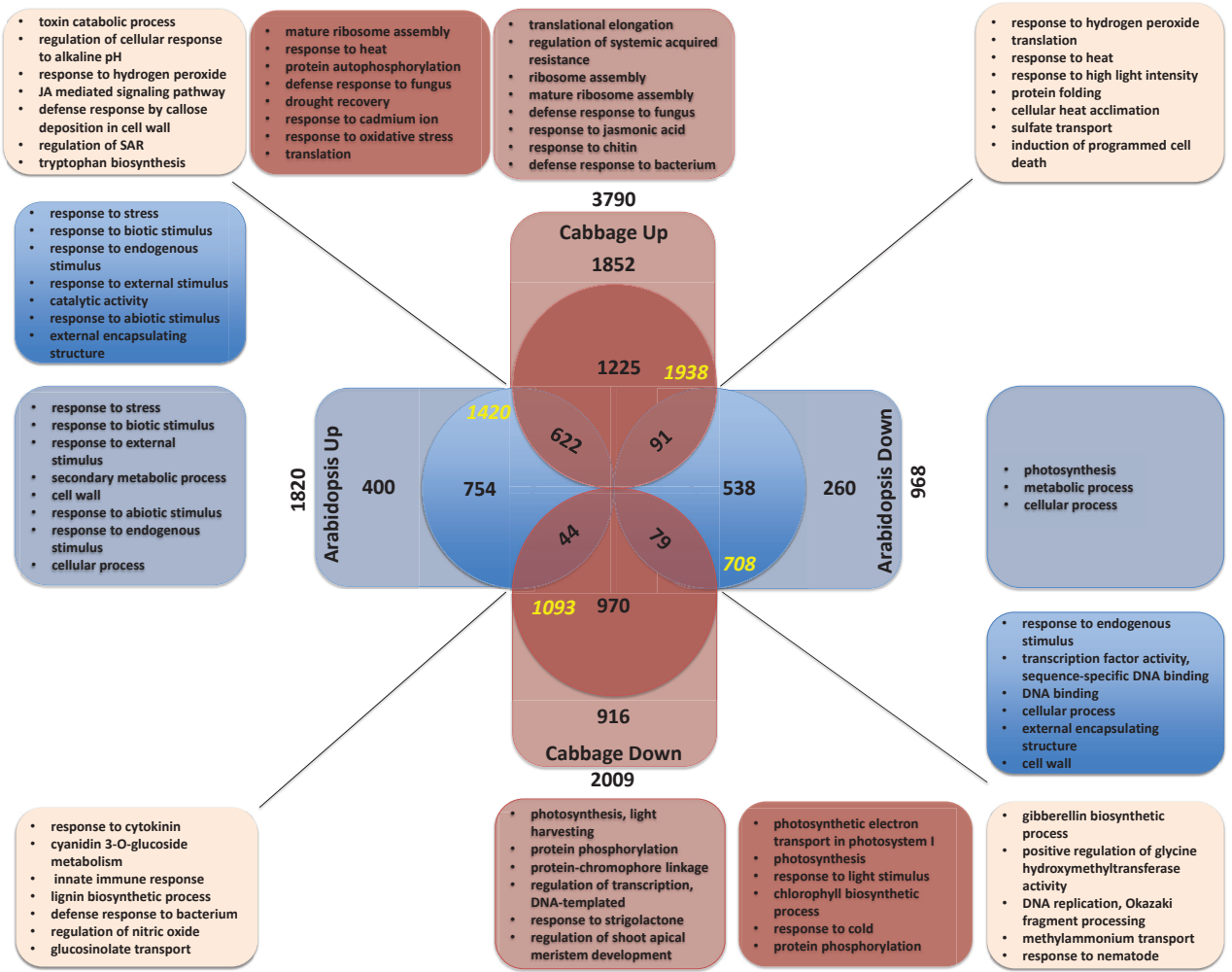

Figure 6. Venn diagram depicting the numbers of genes and biological processes that are similarly or differentially regulated during feeding by western flower thrips on Arabidopsis and by onion thrips feeding on white-cabbage plants. Selected overrepresented GO terms are depicted for each subset of genes. Brown and blue colours represent white cabbage and Arabidopsis DEGs, respectively. Dark blue and dark brown segments represent genes with orthologues in the other plant species, light blue and light brown represent genes without orthologues in the other plant species. Yellow numbers depict total numbers of genes with orthologues in the other plant species for each subset of genes.

GO term analysis for each subset of genes was performed for both comparisons (white cabbage vs Arabidopsis and white cabbage vs sweet pepper) (Fig. 6 and 7). Common upregulated genes (622 in white cabbage vs Arabidopsis and 315 in white cabbage vs sweet pepper) from both comparisons are associated with JA-related GO terms, such as "JA mediated signalling pathway" and "Response to JA". This exhibits the conservation and importance of the JA pathway in all three plant species against thrips feeding. Moreover, in the white cabbage vs sweet pepper comparison, the sweet pepper DEGs (624) with orthologues in white cabbage, that are species-specifically induced in sweet pepper are associated with the GO term "Isoprenoid (terpenoid) biosynthesis", indicating that this pathway is especially important in sweet 
pepper. In downregulated genes for both comparisons, GO terms are largely associated with development-related processes, such "Photosynthesis", "Photosynthetic electron transport in photosystem I", "Photosynthesis, light harvest", "Chlorophyll biosynthetic process" (Fig. 6 and 7). This indicates that downregulation of developmental processes occurs in all three plant-thrips interactions.

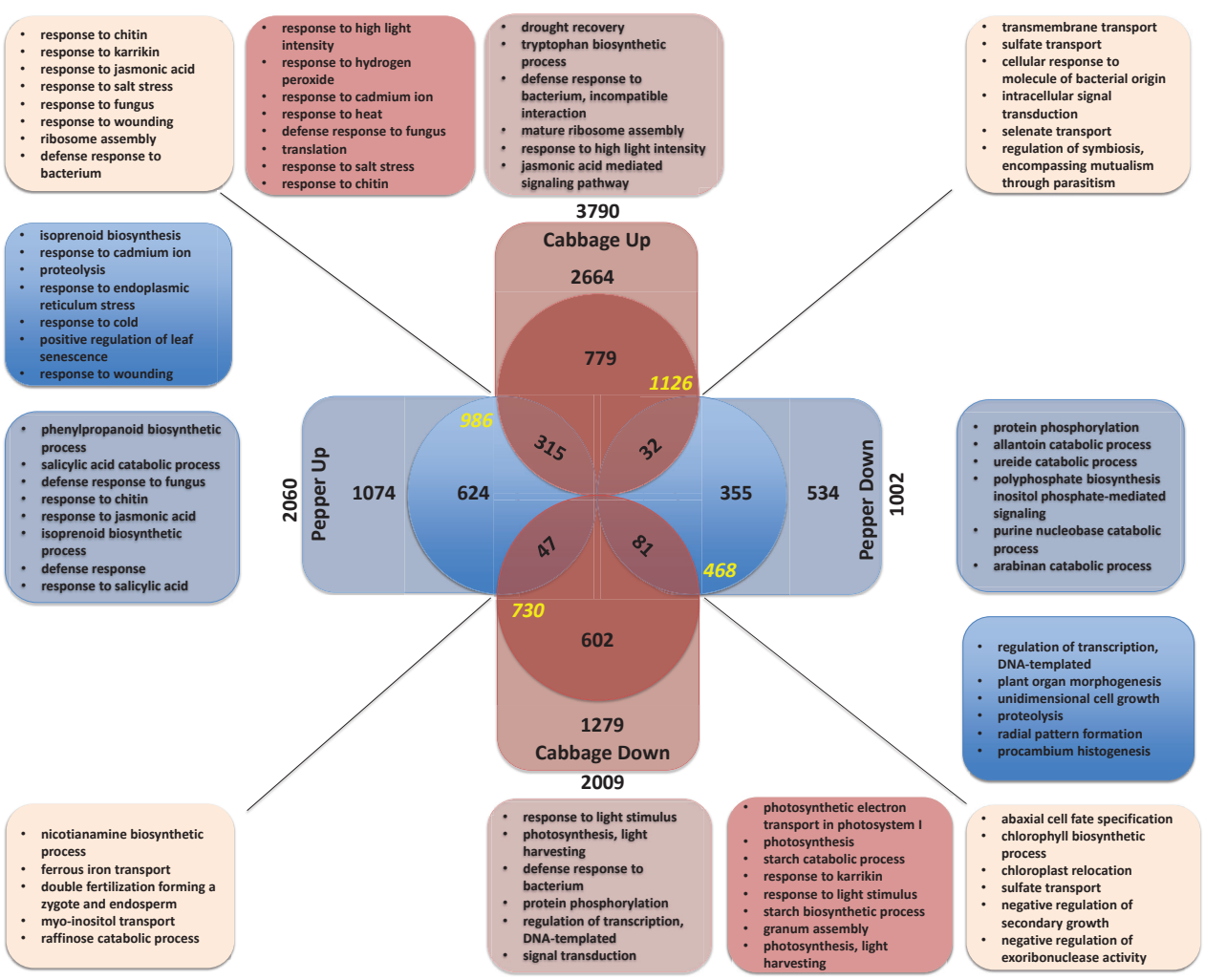

Figure 7. Venn diagram depicting the numbers of genes and biological processes that are similarly or differentially regulated during feeding by western flower thrips on sweet pepper plants and by onion thrips feeding on white cabbage plants. Selected overrepresented GO terms are depicted for each subset of genes. Dark blue and dark brown segments represent genes with orthologues in the other plant species, light blue and light brown represent genes without orthologues in the other plant species. Yellow numbers depict total numbers of genes with orthologues in the other plant species for each subset of genes.

\section{Discussion}

In the present study, we aimed to gain comprehensive insights into the temporally dynamic full-genomic transcriptional response of white cabbage plants to feeding by onion thrips and to elucidate the level of conservation in induced defences in different plants, induced by two thrips species. We recorded that $9.7 \%(5799 / 59225)$ of the 
white cabbage genes is differentially expressed within $8 \mathrm{~h}$ of onion thrips feeding and that the transcriptional patterns of these 5799 genes can be categorized into 48 co-expressed gene clusters (32 up- and 16 downregulated). The up- and downregulated gene clusters are broadly associated with defence and development-related biological processes, respectively. The majority of transcriptional reprogramming occurs already within $2 \mathrm{~h}$ after the start of exposure to thrips. Processes induced include the biosynthesis of phytohormones (JA, ET and SA) and secondary metabolites (phenylpropanoids, flavonoids, GLVs and indolic GLS), whereas developmental processes and the aliphatic GLS pathway are suppressed.

Through a similar protocol, we have previously also analysed the full-transcriptomic responses of Arabidopsis and sweet pepper plants to WFT (Sarde et al. 2019; Steenbergen et al. 2019). A comparative transcriptomic analysis between the onion-thrips-induced white cabbage transcriptome and the WFT-induced Arabidopsis and sweet pepper transcriptomes showed 1) that the induction of JA biosynthesis and signalling is conserved in all three plant species, 2) that white cabbage responds rapidly with a relatively complex temporal transcriptional response (48 clusters), whereas the sweet pepper and Arabidopsis responses are somewhat slower and characterized by approximately half the number of clusters, i.e. 23 and 20 clusters respectively, 3) that TFs like MYB, bHLH and WRKY are conserved as major regulators of the response to thrips feeding, and 4) that the majority of the full-genome transcriptional responses against thrips are system-specific.

\section{Majority of transcriptomic responses to thrips are system-specific}

In both transcriptomic comparisons (white-cabbage vs Arabidopsis and white cabbage vs sweet pepper), a similar pattern in terms of the distribution of orthologous genes over the three categories (a) similarly regulated, (b) oppositely regulated and (c) differentially regulated in only one of the two species, was observed (Fig. 6 and 7). The emerging pattern is that: 1) there is more overlap in upregulated orthologous genes than in downregulated orthologous genes and 2) the majority of DEGs from each plant species shows species-specific differential expression. A similar conclusion was drawn for a comparison of Arabidopsis vs tomato plants upon infestation by the spider mite Tetranychus urticae, another cell-content feeder (Martel et al., 2015) and the comparison of the Arabidopsis and sweet pepper transcriptomes in response to WFT feeding (Sarde et al., 2019; Steenbergen et al., 2019). Moreover, in the comparison between white cabbage (Brassicaceae) and Arabidopsis (Brassicaceae) a larger number of orthologues and common genes for each subset were found than in the comparison between white cabbage (Brassicaceae) and sweet pepper (Solanaceae). This suggests that the transcriptional responses are mostly plant-family specific, irrespective of different thrips species feeding. Moreover, the common subset 
of upregulated genes from both comparisons represents $\mathrm{GO}$ terms associated with JA-related biological processes ("JA mediated signalling pathway" and "Response to JA"), validating conservation of JA-regulated defences in Arabidopsis, white-cabbage and sweet pepper, although elicited by two different thrips species. Overall, this analysis suggests a conservation of the JA pathway in all three plants, the presence of more commonalities in induced defences than suppressed processes and the majority of induced responses against thrips being system-specific.

\section{Conserved activation of hormonal and secondary metabolite pathways upon thrips feeding}

In plant-insect interactions, phytohormones are central players in plant responses to feeding damage (De Vos et al., 2005; Pieterse et al., 2009; Verhage et al., 2010; Pieterse et al., 2012; Stam et al., 2014). For example, JA is conserved in mediating responses against chewing caterpillars (Reymond et al., 2004; De Vos et al., 2005) and cell-content feeding insects like thrips (Abe et al., 2008; Abe et al., 2012; Sarde et al., 2018a; Steenbergen et al., 2018), whereas SA mediates responses against phloem-feeding insects like aphids and whiteflies (Zhu-Salzman et al., 2004; Walling, 2008; Pieterse et al., 2012; Tzin et al., 2015; Broekgaarden et al., 2018). In the present study, through comparative analysis, we show that both JA and ET hormonal pathways are induced in all three plant species (Table 1). This suggests conservation of synergism between the JA and ET hormonal pathways in fine tuning the responses to thrips. Moreover, several studies have shown induction of the SA pathway upon thrips feeding, but at later time points (Abe et al., 2008; Sarde et al., 2018a). In our studies, the SA pathway is also induced later in the brassicaceous plants Arabidopsis and white cabbage and supressed in sweet pepper during the first $8 \mathrm{~h}$ of thrips feeding. The relatively slow transcriptional response of sweet pepper may imply that the SA pathway is induced at later time points beyond the $8 \mathrm{~h}$ time window of the study (Sarde et al. 2019). The phenylpropanoid and flavonoid pathways are induced in all three plant species upon thrips feeding (Table 1), suggesting that these pathways may be involved in plant defence against thrips, as reported for defences against other insect herbivores (Mallikarjuna et al., 2004; Misra et al., 2010; Onkokesung et al., 2014).

\section{Potential defensive role of indolic GLS in white cabbage against onion thrips}

Although the role of glucosinolates in plant defence responses against herbivory is well studied, relatively little is known about their role against cell-content feeders, such as thrips. Glucosinolates, are a Brassicaceae-specific group of secondary metabolites. Upon herbivore feeding, the production of GLS is induced, resulting in interference with the performance of herbivorous insects. Different types of GLS are induced upon herbivory by different species, subsequently affecting their performance. 
For example, indolic GLS affect the performance of phloem feeders (Elbaz et al., 2012; Markovich et al., 2013; Zust \& Agrawal, 2016), whereas, aliphatic GLS affect the performance of chewing insects (Schweizer et al., 2013). Our transcriptional data show that all genes involved in the regulation and biosynthesis of indolic GLS are induced. In contrast, the main upstream regulatory gene of aliphatic GLS biosynthesis, BoMYB28, is downregulated and none of the genes involved in biosynthesis of aliphatic GLS showed differential expression upon thrips feeding. A similar pattern of induction in indolic GLS and suppression of aliphatic GLS is seen in the WFT-induced Arabidopsis transcriptome (Fig. S2). This suggests specific induction of indolic GLS in brassicaceous plants by thrips feeding and indicates their potential role in defence against them. Similar observations of specific induction of indolic GLS and their defensive role are reported in Arabidopsis in response to another cell-content feeder, the two-spotted spider mite Tetranychus urticae (Zhurov et al., 2014). These data suggest that the induction of indolic GLS upon damage by cell-content feeders and their potential defensive role is conserved in different brassicaceous species.

\section{Rapid and complex transcriptional response of white cabbage to onion thrips feeding}

Plants respond to different stresses by dynamically reprogramming their transcriptome (Windram et al., 2012; Lewis et al., 2015; Hickman et al., 2017; Sarde et al., 2019; Steenbergen et al., 2019). In plant-insect interactions, the speed and complexity of the transcriptional response of plants depends on many characteristics of the attacking herbivore, such as feeding guild, density of herbivores and timing of attack (Heidel-Fischer et al., 2014; Stam et al., 2014). Until now, several studies on plant transcriptomic responses to different herbivore species analysed the response at only one or two time points spread over a period of $24 \mathrm{~h}$ or longer (De Vos et al., 2005; Ehlting et al., 2008; Bidart-Bouzat \& Kliebenstein, 2011; Zhang et al., 2013; Appel et al., 2014; Diaz-Riquelme et al., 2016; Kroes et al., 2017; Broekgaarden et al., 2018; Tu et al., 2018). The main focus of these studies was to understand the overall response to feeding by the respective herbivore. In the present study, by comparing the high-density whole-genome transcriptional response of three plant species (Arabidopsis, sweet pepper and white cabbage) to thrips feeding, we show that white cabbage is relatively fast in its overall transcriptional response (Table 1). In white cabbage, the majority of the DEGs are induced within 1-2 h (Fig. 5 and 8), whereas in Arabidopsis and sweet pepper this is later than 2-3 h of thrips feeding (Fig. 8) (Sarde et al., 2019; Steenbergen et al., 2019). The rapid response of white cabbage comprises genes involved in the biosynthesis of phytohormones and secondary metabolites (Fig. 3, 4 and Table 1). Whether this is a plant-specific characteristic or a thrips-specific characteristic remains to be investigated. If it is a thrips-specific characteristic, the relatively rapid response of white cabbage against onion thrips compared to Arabidopsis and 
sweet pepper against WFT feeding might be due to two main reasons: 1) onion thrips effectors are less effective in suppressing the initial defence response of white cabbage or, 2) onion thrips feed faster than WFT. Moreover, we found more temporally dynamic transcriptional patterns (genes in clusters with similar expression pattern) among the DEGs of white cabbage (48 clusters), compared to Arabidopsis (20 clusters) (Steenbergen et al., 2019) and sweet pepper (23 clusters) (Sarde et al., 2019). The larger number of temporally dynamic transcriptional patterns may be indicative of the complexity of the transcriptional response of white cabbage to onion thrips feeding. This relatively complex transcriptional response of white cabbage could be a result of its evolution from multiple ancestral polyploidy events or from an overall larger number of genes (Parkin et al., 2014). Taken together, this comparative analysis suggests that white cabbage responds rapidly with a complex transcriptional pattern to onion thrips feeding.

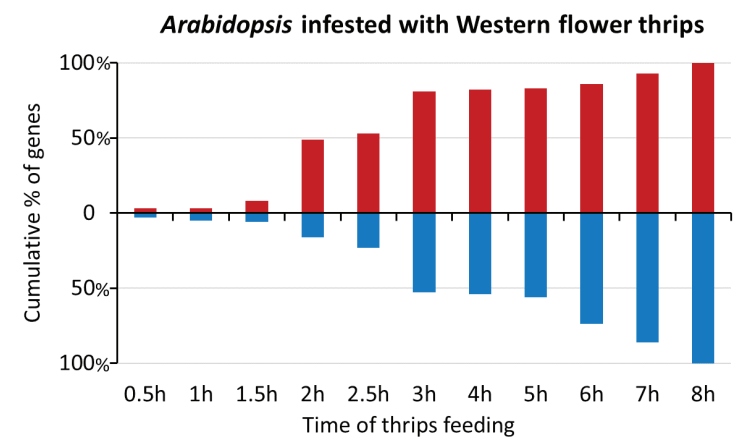

Sweet pepper infested with Western flower thrips

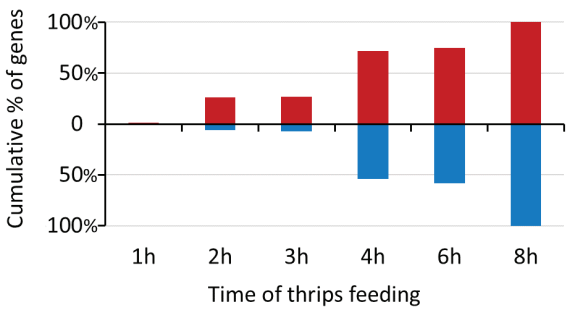

White cabbage infested with Onion thrips

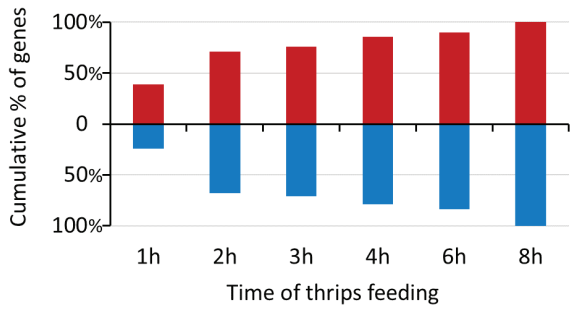

Figure 8. Whole-genome transcriptional response of Arabidopsis, white cabbage and sweet pepper in response to thrips feeding. $100 \%$ is 2788 (1820 up- and 968 downregulated) genes for Arabidopsis, 3062 (2060 up- and 1002 downregulated) genes for sweet pepper and 5799 (3790 up- and 2009 downregulated) genes for white cabbage. 


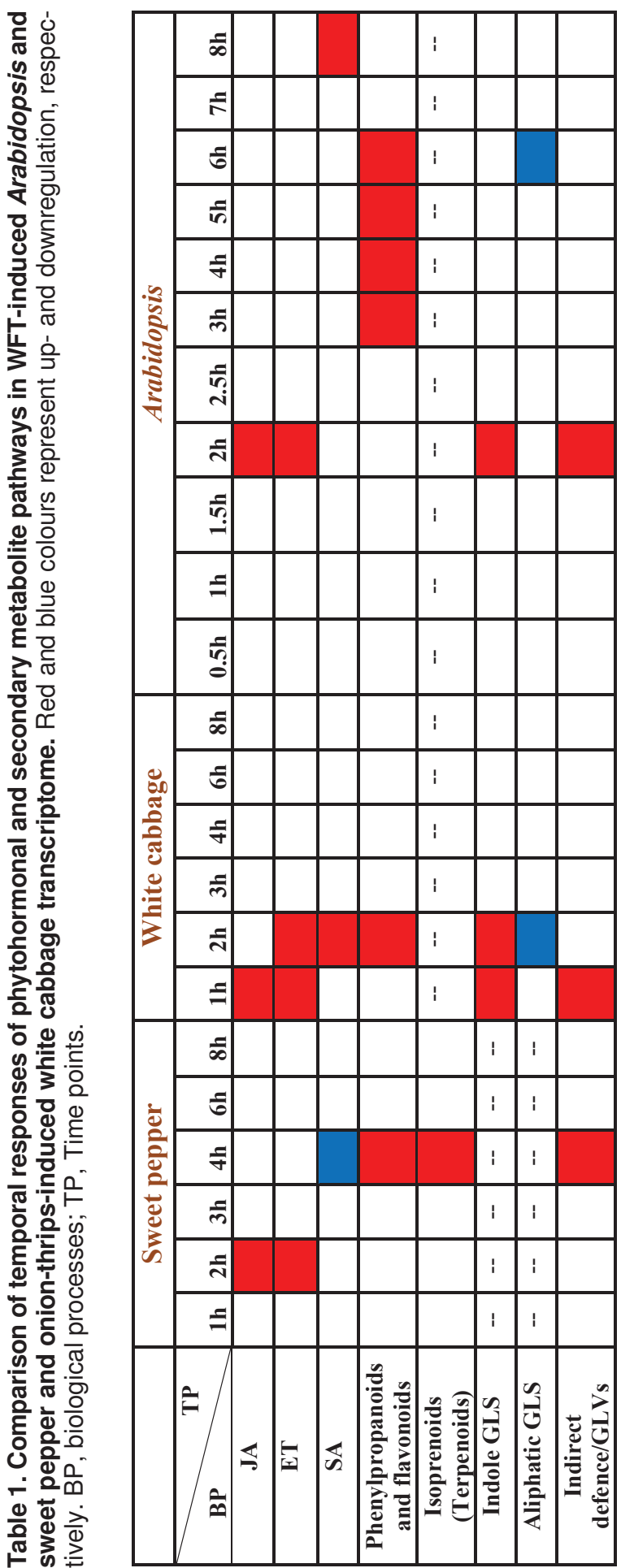




\section{Conservation of bHLH, MYB and WRKY TFs as major regulators upon thrips feeding}

TFs modulate the transcriptional reprogramming of plants in response to different stresses (Breeze et al., 2011; Windram et al., 2012; Jin et al., 2017; Sarde et al., 2019; Steenbergen et al., 2019). In the white cabbage transcriptomic response to onion thrips and in the Arabidopsis and sweet pepper transcriptomic responses to WFT, we found TF families such as bHLH, MYB and WRKY being overrepresented in all three plant species. Moreover, MYB and bHLH TFs are also found to be overrepresented in the Arabidopsis transcriptomic response to exogenous MeJA application (Hickman et al., 2017). This suggests that these TF families are conserved in all three plant species in regulating the defences against thrips species mediated by JA. Additionally, in all three plant species, through motif enrichment analysis, bHLH TFs are found to be enriched in gene clusters overrepresented in JA-related biological processes (Sarde et al., 2019; Steenbergen et al., 2019). This supports the role of the bHLH TF family in regulating JA signalling processes (Goossens et al., 2017).

\section{Conclusion}

High-density transcriptomic analysis of the onset of plant-thrips interactions reveals the complexity of responses of three plant species to feeding by these herbivores. Almost a tenth of a plant's genome changes transcription level in with many groups of genes that share their dynamic expression pattern. The transcriptional response is highly specific for each plant-thrips interaction. More similarities were recorded among upregulated genes than among downregulated genes. The three major phytohormones, JA, SA and ET show similar expression patterns albeit at different temporal scales. Similarities among upregulated genes were especially related to JA biosynthesis and responses. This detailed comparative transcriptomic analysis of early molecular processes underlying plant-thrips interactions underlines the complex choreography of induced plant responses to insect feeding.

\section{Acknowledgements}

We thank Keygene (Wageningen, The Netherlands) for RNA sequencing, Betty Henken for providing the space and help to rear Onion thrips, Wageningen University \& Research experimental farm (Unifarm) for rearing the plants, and colleagues from the Laboratory of Entomology (Wageningen University) for help in sampling leaf tissues for RNA-Seq analysis. This work is part of the Perspective programme Green Defences Against Pests that is (partly) financed by the Netherlands Organisation for Scientific Research (NWO, grant no. 13555) and partly by the breeding companies 
Keygene, Syngenta and East-West Seed through NWO. RH was funded by NWO VENI grant no. 13682

\section{References}

Abe H, Ohnishi J, Narusaka M, Seo S, Narusaka Y, Tsuda S, Kobayashi M. 2008. Function of jasmonate in response and tolerance of Arabidopsis to thrip feeding. Plant Cell Physiology 49(1): 68-80.

Abe H, Tomitaka Y, Shimoda T, Seo S, Sakurai T, Kugimiya S, Tsuda S, Kobayashi M. 2012. Antagonistic plant defense system regulated by phytohormones assists interactions among vector insect, Thrips and a Tospovirus. Plant and Cell Physiology 53(1): 204-212.

Ali S, Mir ZA, Tyagi A, Bhat JA, Chandrashekar N, Papolu PK, Rawat S, Grover A. 2017. Identification and comparative analysis of Brassica juncea pathogenesis-related genes in response to hormonal, biotic and abiotic stresses. Acta Physiologiae Plantarum 39(12): 268.

Anders S, Pyl PT, Huber W. 2015. HTSeq-a python framework to work with high-throughput sequencing data. Bioinformatics 31(2): 166-169.

Appel HM, Fescemyer H, Ehlting J, Weston D, Rehrig E, Joshi T, Xu D, Bohlmann J, Schultz J. 2014. Transcriptional responses of Arabidopsis thaliana to chewing and sucking insect herbivores. Frontiers in Plant Science 5: 565.

Bate NJ, Sivasankar S, Moxon C, Riley JMC, Thompson JE, Rothstein SJ. 1998. Molecular characterization of an Arabidopsis gene encoding hydroperoxide lyase, a cytochrome P-450 that is wound inducible. Plant Physiology 117(4): 1393-1400.

Bidart-Bouzat MG, Kliebenstein D. 2011. An ecological genomic approach challenging the paradigm of differential plant responses to specialist versus generalist insect herbivores. Oecologia 167(3): 677-689.

Bolger AM, Lohse M, Usadel B. 2014. Trimmomatic: a flexible trimmer for Illumina sequence data. Bioinformatics 30(15): 2114-2120.

Breeze E, Harrison E, McHattie S, Hughes L, Hickman R, Hill C, Kiddle S, Kim YS, Penfold CA, Jenkins D, Zhang CJ, Morris K, Jenner C, Jackson S, Thomas B, Tabrett A, Legaie R, Moore JD, Wild DL, Ott S, Rand D, Beynon J, Denby K, Mead A, Buchanan-Wollaston V. 2011. High-resolution temporal profiling of transcripts during Arabidopsis leaf senescence reveals a distinct chronology of processes and regulation. Plant Cell 23(3): 873-894.

Broekgaarden C, Pelgrom KTB, Bucher J, van Dam NM, Grosser K, Pieterse CMJ, van Kaauwen M, Steenhuis G, Voorrips RE, de Vos M, Vosman B, Worrich A, van Wees SCM. 2018. Combining QTL mapping with transcriptome and metabolome profiling reveals a possible role for ABA signaling in resistance against the cabbage whitefly in cabbage. PLoS One 13(11): e0206103.

De Vos M, Van Oosten VR, Van Poecke RMP, Van Pelt JA, Pozo MJ, Mueller MJ, Buchala AJ, Metraux JP, Van Loon LC, Dicke M, Pieterse CMJ. 2005. Signal signature and transcriptome changes of Arabidopsis during pathogen and insect attack. Molecular Plant-Microbe Interactions 18(9): 923-937.

Diaz-Montano J, Fuchs M, Nault BA, Fail J, Shelton AM. 2011. Onion thrips (Thysanoptera: Thripidae): A global pest of increasing concern in onion. Journal of Economic Entomology 104(1): 1-13.

Diaz-Riquelme J, Zhurov V, Rioja C, Perez-Moreno I, Torres-Perez R, Grimplet J, Car- 
bonell-Bejerano P, Bajda S, Van Leeuwen T, Martinez-Zapater JM, Grbic M, Grbic V. 2016. Comparative genome-wide transcriptome analysis of Vitis vinifera responses to adapted and non-adapted strains of two-spotted spider mite, Tetranyhus urticae. BMC Genomics 17: 74.

Ehlting J, Chowrira SG, Mattheus N, Aeschliman DS, Arimura GI, Bohlmann J. 2008. Comparative transcriptome analysis of Arabidopsis thaliana infested by diamond back moth (Plutella xylostella) larvae reveals signatures of stress response, secondary metabolism, and signalling. BMC Genomics 9: 154.

Elbaz M, Halon E, Malka O, Malitsky S, Blum E, Aharoni A, Morin S. 2012. Asymmetric adaptation to indolic and aliphatic glucosinolates in the $\mathrm{B}$ and $\mathrm{Q}$ sibling species of Bemisia tabaci (Hemiptera: Aleyrodidae). Molecular Ecology 21(18): 4533-4546.

Fail J, Deutschlander ME, Shelton AM. 2013. Antixenotic resistance of cabbage to onion thrips (Thysanoptera: Thripidae). I. light reflectance. Journal of Economic Entomology 106(6): 2602-2612.

Franco-Zorrilla JM, Lopez-Vidriero I, Carrasco JL, Godoy M, Vera P, Solano R. 2014. DNA-binding specificities of plant transcription factors and their potential to define target genes. Proceedings of the National Academy of Sciences of the United States of America 111(6): 2367-2372.

Frerigmann H, Pislewska-Bednarek M, Sanchez-Vallet A, Molina A, Glawischnig E, Gigolashvili T, Bednarek P. 2016. Regulation of pathogen-triggered tryptophan metabolism in Arabidopsis thaliana by $M Y B$ transcription factors and indole glucosinolate conversion products. Molecular Plant 9(5): 682-695.

Gill HK, Garg H, Gill AK, Gillett-Kaufman JL, Nault BA. 2015. Onion thrips (Thysanoptera: Thripidae) biology,ecology, and management in onion production systems. Journal of Integrated Pest Management 6(1): 10.1093/jipm/pmv1006.

Goossens J, Mertens J, Goossens A. 2017. Role and functioning of bHLH transcription factors in jasmonate signalling. Journal of Experimental Botany 68(6): 1333-1347.

Grant CE, Bailey TL, Noble WS. 2011. FIMO: scanning for occurrences of a given motif. Bioinformatics 27(7): 1017-1018.

Halkier BA, Gershenzon J. 2006. Biology and biochemistry of glucosinolates. Annual Review of Plant Biology 57: 303-333.

Heard NA. 2011. Iterative reclassification in agglomerative clustering. J. Comput. Graph. Stat. 20: $920-936$.

Heard NA, Holmes CC, Stephens DA. 2006. A quantitative study of gene regulation involved in the immune response of anopheline mosquitoes: An application of Bayesian hierarchical clustering of curves. J. Am. Stat. Assoc. 101: 18-29.

Heidel-Fischer HM, Musser RO, Vogel H 2014. Plant transcriptomic responses to herbivory. Annual Plant Reviews: John Wiley \& Sons, Ltd, 155-196.

Hickman R, Van Verk MC, Van Dijken AJH, Mendes MP, Vroegop-Vos IA, Caarls L, Steenbergen M, Van der Nagel I, Wesselink GJ, Jironkin A, Talbot A, Rhodes J, De Vries M, Schuurink RC, Denby K, Pieterse CMJ, Wees SCM. 2017. Architecture and dynamics of the jasmonic acid gene regulatory network. Plant Cell 29(9): 2086-2105.

Ishida M, Hara M, Fukino N, Kakizaki T, Morimitsu Y. 2014. Glucosinolate metabolism, functionality and breeding for the improvement of Brassicaceae vegetables. Breeding Science 64(1): 48-59.

Jin JP, Tian F, Yang DC, Meng YQ, Kong L, Luo JC, Gao G. 2017. PlantTFDB 4.0: toward a central hub for transcription factors and regulatory interactions in plants. Nucleic Acids 
Research 45(D1): D1040-D1045.

Kim D, Pertea G, Trapnell C, Pimentel H, Kelley R, Salzberg SL. 2013. TopHat2: accurate alignment of transcriptomes in the presence of insertions, deletions and gene fusions. Genome Biology 14(4): R36.

Klopfenstein DV, Zhang LS, Pedersen BS, Ramirez F, Vesztrocy AW, Naldi A, Mungall CJ, Yunes JM, Botvinnik O, Weigel M, Dampier W, Dessimoz C, Flick P, Tang HB. 2018. GOATOOLS: A python library for gene ontology analyses. Scientific Reports 8: 10.1038/ s41598-41018-28948-z.

Kroes A, Broekgaarden C, Uribe MC, May S, van Loon JJA, Dicke M. 2017. Brevicoryne brassicae aphids interfere with transcriptome responses of Arabidopsis thaliana to feeding by Plutella xylostella caterpillars in a density-dependent manner. Oecologia 183(1): 107-120.

Lewis LA, Polanski K, de Torres-Zabala M, Jayaraman S, Bowden L, Moore J, Penfold CA, Jenkins DJ, Hill C, Baxter L, Kulasekaran S, Truman W, Littlejohn G, Prusinska J, Mead A, Steinbrenner J, Hickman R, Rand D, Wild DL, Ott S, Buchanan-Wollaston V, Smirnoff N, Beynon J, Denby K, Grant M. 2015. Transcriptional dynamics driving MAMP-triggered immunity and pathogen effector-mediated immunosuppression in Arabidopsis leaves following infection with Pseudomonas syringae pv tomato DC3000. Plant Cell 27(11): 3038-3064.

Love MI, Anders S, Kim V, Huber W. 2015. RNA-Seq workflow: gene-level exploratory analysis and differential expression. F1000Res 4: 1070.

Love MI, Huber W, Anders S. 2014. Moderated estimation of fold change and dispersion for RNA-seq data with DESeq2. Genome Biology 15(12): 550.

Maffei ME, Mithofer A, Boland W. 2007. Before gene expression: early events in plant-insect interaction. Trends in Plant Science 12(7): 310-316.

Mallikarjuna N, Kranthi KR, Jadhav DR, Kranthi S, Chandra S. 2004. Influence of foliar chemical compounds on the development of Spodoptera litura (Fab.) in interspecific derivatives of groundnut. Journal of Applied Entomology 128(5): 321-328.

Markovich O, Kafle D, Elbaz M, Malitsky S, Aharoni A, Schwarzkopf A, Gershenzon J, Morin S. 2013. Arabidopsis thaliana plants with different levels of aliphatic- and indolyl-glucosinolates affect host selection and performance of Bemisia tabaci. Journal of Chemical Ecology 39(11-12): 1361-1372.

Martel C, Zhurov V, Navarro M, Martinez M, Cazaux M, Auger P, Migeon A, Santamaria ME, Wybouw N, Diaz I, Van Leeuwen T, Navajas M, Grbic M, Grbic V. 2015. Tomato whole genome transcriptional response to Tetranychus urticae identifies divergence of spider mite-induced responses between tomato and Arabidopsis. Molecular Plant-Microbe Interactions 28(3): 343-361.

Misra P, Pandey A, Tiwari M, Chandrashekar K, Sidhu OP, Asif MH, Chakrabarty D, Singh PK, Trivedi PK, Nath P, Tuli R. 2010. Modulation of transcriptome and metabolome of tobacco by Arabidopsis transcription factor, AtMYB12, leads to insect resistance. Plant Physiology 152(4): 2258-2268.

Onkokesung N, Reichelt M, van Doorn A, Schuurink RC, van Loon JJA, Dicke M. 2014. Modulation of flavonoid metabolites in Arabidopsis thaliana through overexpression of the MYB75 transcription factor: role of kaempferol-3,7-dirhamnoside in resistance to the specialist insect herbivore Pieris brassicae. Journal of Experimental Botany 65(8): 22032217.

Parkin IAP, Koh C, Tang HB, Robinson SJ, Kagale S, Clarke WE, Town CD, Nixon J, Krishnakumar V, Bidwell SL, Denoeud F, Belcram H, Links MG, Just J, Clarke C, 
Bender T, Huebert T, Mason AS, Pires JC, Barker G, Moore J, Walley PG, Manoli S, Batley J, Edwards D, Nelson MN, Wang XY, Paterson AH, King G, Bancroft I, Chalhoub B, Sharpe AG. 2014. Transcriptome and methylome profiling reveals relics of genome dominance in the mesopolyploid Brassica oleracea. Genome Biology 15(6): R77.

Pieterse CMJ, Leon-Reyes A, Van der Ent S, Van Wees SCM. 2009. Networking by small-molecule hormones in plant immunity. Nature Chemical Biology 5(5): 308-316.

Pieterse CMJ, Van der Does D, Zamioudis C, Leon-Reyes A, Van Wees SCM. 2012. Hormonal modulation of plant immunity. Annual Review of Cell and Developmental Biology 28: 489-521.

Poelman EH, Van Loon JJA, Van Dam NM, Vet LEM, Dicke M. 2010. Herbivore-induced plant responses in Brassica oleracea prevail over effects of constitutive resistance and result in enhanced herbivore attack. Ecological Entomology 35(2): 240-247.

Reymond P, Bodenhausen N, Van Poecke RM, Krishnamurthy V, Dicke M, Farmer EE. 2004. A conserved transcript pattern in response to a specialist and a generalist herbivore. Plant Cell 16(11): 3132-3147.

Sarde S, Hickman R, Steenbergen M, Wiegers G, Pieterse CMJ, Van Wees SCM, Dicke M. 2019. Whole-genome transcriptional reprogramming of sweet pepper in response to Western flower thrips feeding. In prep.

Sarde SJ, Bouwmeester K, Venegas-Molina J, David A, Boland W, Dicke M. 2018a. Involvement of sweet pepper CaLOX2 in jasmonate-dependent induced defence against Western flower thrips. Journal of Integrative Plant Biology: 10.1111/jipb.12742.

Sarde SJ, Kumar A, Remme RN, Dicke M. 2018b. Genome-wide identification, classification and expression of lipoxygenase gene family in pepper. Plant Molecular Biology: 10.1007/ s11103-11018-10785-y.

Schoonhoven LM, van Loon JJA, Dicke M. 2005. Insect-Plant Biology, Ed 2: Oxford University Press, Oxford.

Schweizer F, Fernandez-Calvo P, Zander M, Diez-Diaz M, Fonseca S, Glauser G, Lewsey MG, Ecker JR, Solano R, Reymond P. 2013. Arabidopsis basic helix-loop-helix transcription factors $M Y C 2, M Y C 3$, and MYC4 regulate glucosinolate biosynthesis, insect performance, and feeding behavior. Plant Cell 25(8): 3117-3132.

Shannon P, Markiel A, Ozier O, Baliga NS, Wang JT, Ramage D, Amin N, Schwikowski B, Ideker T. 2003. Cytoscape: A software environment for integrated models of biomolecular interaction networks. Genome Research 13(11): 2498-2504.

Shelton AM, Plate J, Chen M. 2008. Advances in control of onion thrips (Thysanoptera : Thripidae) in cabbage. Journal of Economic Entomology 101(2): 438-443.

Stam JM, Dicke M, Poelman EH. 2018. Order of herbivore arrival on wild cabbage populations influences subsequent arthropod community development. Oikos 127(10): 1482-1493.

Stam JM, Kroes A, Li YH, Gols R, van Loon JJA, Poelman EH, Dicke M. 2014. Plant interactions with multiple insect herbivores: From community to genes. Annual Review of Plant Biology 65: 689-713.

Steenbergen M, Abd-el-Haliem A, Bleeker P, Dicke M, Escobar-Bravo R, Cheng G, Haring MA, Kant MR, Kappers I, Klinkhamer PGL, Leiss KA, Legarrea S, Macel M, Mouden S, Pieterse CMJ, Sarde SJ, Schuurink RC, De Vos M, Van Wees SCM, Broekgaarden C. 2018. Thrips advisor: exploiting thrips-induced defences to combat pests on crops. Journal of Experimental Botany 69(8): 1837-1848. 
Steenbergen M, Hickman R, Sarde S, Dicke M, Pieterse CMJ, Van Wees SCM. 2019. High-resolution temporal transcriptomic dynamics of Arabidopsis thaliana in response to Western flower thrips feeding. In prep.

Tu XB, Liu ZK, Zhang ZH. 2018. Comparative transcriptomic analysis of resistant and susceptible alfalfa cultivars (Medicago sativa L.) after thrips infestation. BMC Genomics 19: 10.1186/s12864-018-4495-2.

Tytgat TO, Verhoeven KJ, Jansen JJ, Raaijmakers CE, Bakx-Schotman T, Mclntyre LM, van der Putten WH, Biere A, van Dam NM. 2013. Plants know where it hurts: root and shoot jasmonic acid induction elicit differential responses in Brassica oleracea. PLoS One 8(6): e65502.

Tzin V, Fernandez-Pozo N, Richter A, Schmelz EA, Schoettner M, Schafer M, Ahern KR, Meihls LN, Kaur H, Huffaker A, Mori N, Degenhardt J, Mueller LA, Jander G. 2015. Dynamic maize responses to aphid feeding are revealed by a time series of transcriptomic and metabolomic assays. Plant Physiology 169(3): 1727-1743.

Tzin V, Galili G. 2010. The biosynthetic pathways for shikimate and aromatic amino acids in Arabidopsis thaliana. Arabidopsis Book 8: e0132.

Verhage A, van Wees SCM, Pieterse CMJ. 2010. Plant immunity: It's the hormones talking, but what do they say? Plant Physiology 154(2): 536-540.

Walling LL. 2008. Avoiding effective defenses: Strategies employed by phloem-feeding insects. Plant Physiology 146(3): 859-866.

Weirauch MT, Yang A, Albu M, Cote AG, Montenegro-Montero A, Drewe P, Najafabadi HS, Lambert SA, Mann I, Cook K, Zheng H, Goity A, van Bakel H, Lozano JC, Galli M, Lewsey MG, Huang EY, Mukherjee T, Chen XT, Reece-Hoyes JS, Govindarajan S, Shaulsky G, Walhout AJM, Bouget FY, Ratsch G, Larrondo LF, Ecker JR, Hughes TR. 2014. Determination and inference of eukaryotic transcription factor sequence specificity. Cell 158(6): 1431-1443.

Wildermuth MC, Dewdney J, Wu G, Ausubel FM. 2001. Isochorismate synthase is required to synthesize salicylic acid for plant defence. Nature 414(6863): 562-565.

Windram O, Madhou P, McHattie S, Hill C, Hickman R, Cooke E, Jenkins DJ, Penfold CA, Baxter L, Breeze E, Kiddle SJ, Rhodes J, Atwell S, Kliebenstein DJ, Kim YS, Stegle O, Borgwardt K, Zhang CJ, Tabrett A, Legaie R, Moore J, Finkenstadt B, Wild DL, Mead A, Rand D, Beynon J, Ott S, Buchanan-Wollaston V, Denby KJ. 2012. Arabidopsis defense against Botrytis cinerea: Chronology and regulation deciphered by high-resolution temporal transcriptomic analysis. Plant Cell 24(9): 3530-3557.

Zhang PJ, Broekgaarden C, Zheng SJ, Snoeren TAL, van Loon JJA, Gols R, Dicke M. 2013. Jasmonate and ethylene signaling mediate whitefly-induced interference with indirect plant defense in Arabidopsis thaliana. New Phytologist 197(4): 1291-1299.

Zhu-Salzman K, Salzman RA, Ahn JE, Koiwa H. 2004. Transcriptional regulation of sorghum defense determinants against a phloem-feeding aphid. Plant Physiology 134(1): 420-431.

Zhurov V, Navarro M, Bruinsma KA, Arbona V, Santamaria ME, Cazaux M, Wybouw N, Osborne EJ, Ens C, Rioja C, Vermeirssen V, Rubio-Somoza I, Krishna P, Diaz I, Schmid M, Gomez-Cadenas A, Van de Peer Y, Grbic M, Clark RM, Van Leeuwen T, Grbic V. 2014. Reciprocal responses in the interaction between Arabidopsis and the cell-content-feeding chelicerate herbivore spider mite. Plant Physiology 164(1): 384-399.

Zust T, Agrawal AA. 2016. Mechanisms and evolution of plant resistance to aphids. Nature Plants 2(1): 16206. 


\section{Supplementary figures}

A
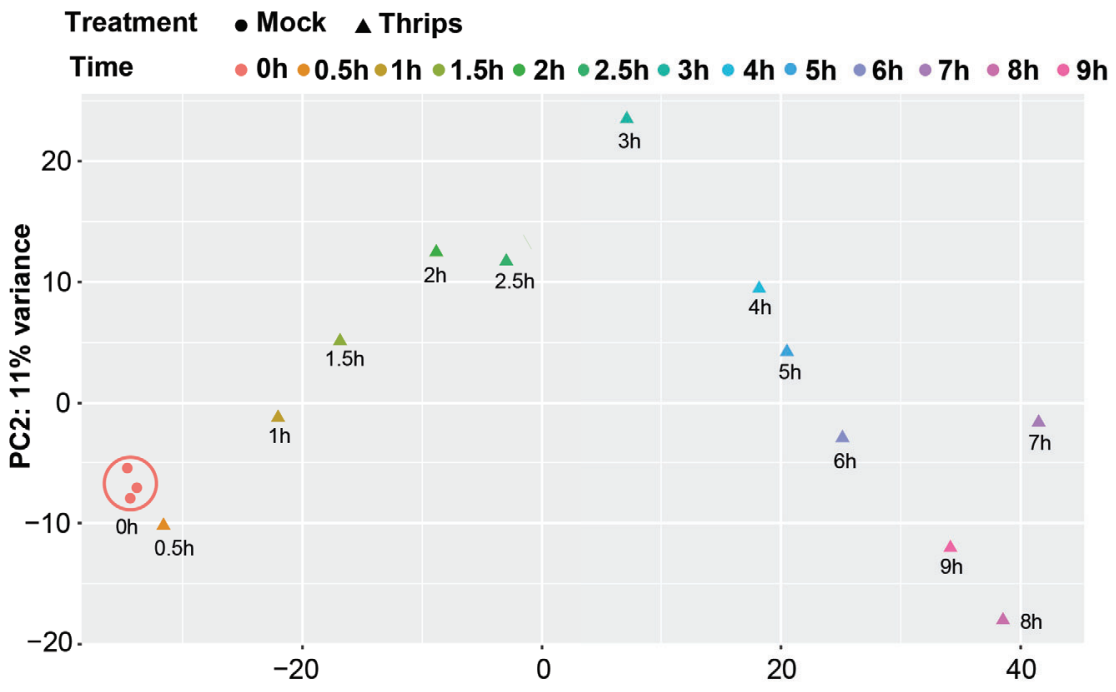

PC1: $73 \%$ variance

B

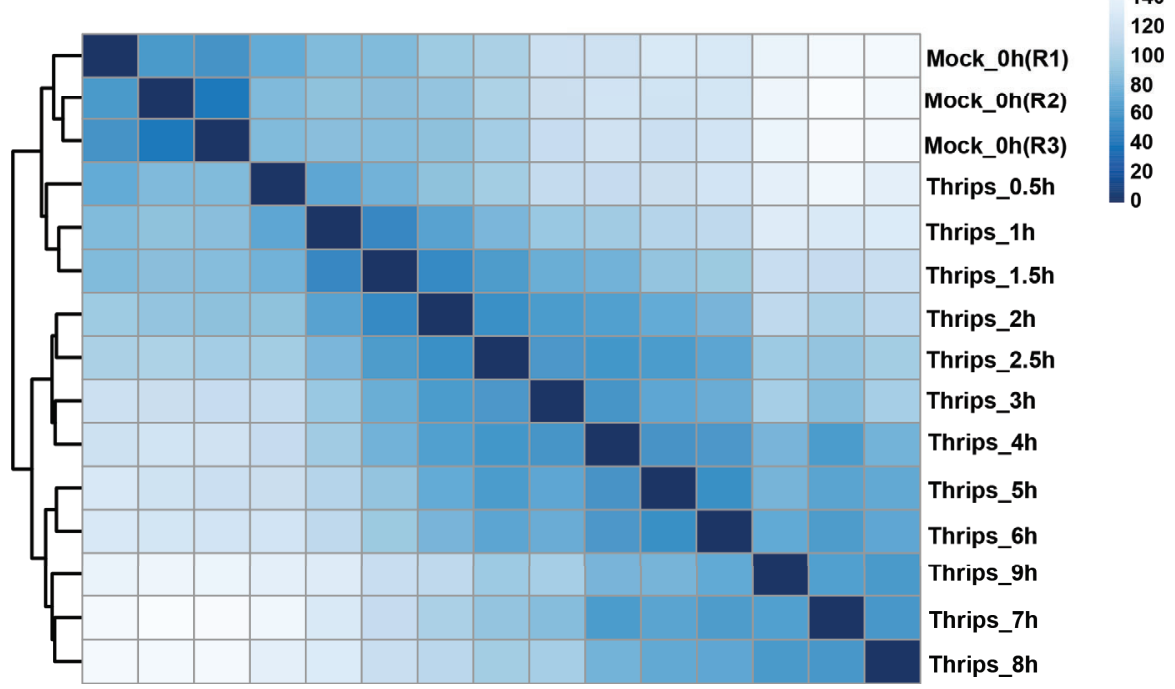

Figure S1. PCA and sample-to-sample distance analysis of transcriptome of white-cabbage infested (thrips) and non-infested (control/mock) plants. (A) PCA plot of sweet pepper non-infested (mock) and infested (thrips) transcriptome. PCA was generated on the regularized $\log _{2}$-transformed data with DESeq2 package in $\mathrm{R}$. Colours and shapes indicate different time points and treatments, respectively. Variation in percentage within the samples is depicted on both axes. (B) Sample-to-sample plot of sweet pepper non-infested (mock) and infested (thrips) transcriptome. It was generated on the regularized $\log _{2}$-transformed data with DESeq2 package in $\mathrm{R}$. 
A

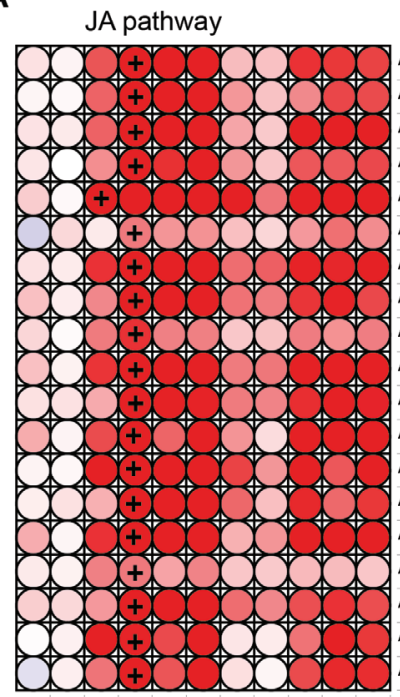

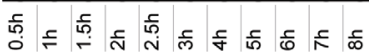

B

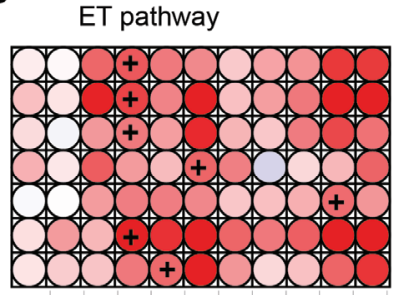

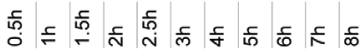

C

SA pathway

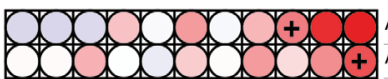

AT2G37040/AtPAL1 AT1G74710/AtICS1

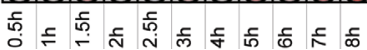
\begin{tabular}{l} 
AT4G01850/SAMS1 \\
AT1G01480/ACS2 \\
\hline AT4G11280/ACS6 \\
AT4G37770/ACS8 \\
\hline AT1G05010/ACO4 \\
\hline AT3G23240/ERF1 \\
AT5G47230/ERF5
\end{tabular}
D

\begin{tabular}{l} 
AT3G45140/AtLOX2 \\
AT5G42650/AAAOS \\
\hline AT3G25760/AtAOC1 \\
\hline AT3G25770/AtAOC2 \\
AT3G25780/AAAOC3 \\
\hline AT1G13280/AAAOC4 \\
\hline AT1G19180/JAZ1 \\
\hline AT1G74950/JAZ2 \\
\hline AT3G1786/JAZ3 \\
\hline AT1G17380/JAZ5 \\
\hline AT1G72450/JAZ6 \\
\hline AT2G34600/JAZ7 \\
\hline AT1G30135/JAZ8 \\
\hline AT1G70700/JAZ9 \\
\hline AT5G13220/JAZ10 \\
\hline AT5G20000/JAZ12 \\
AT1G32640/AtMYC2 \\
\hline AT5G24780/AtVSP1 \\
\hline AT5G24770/AtVSP2 \\
\hline
\end{tabular}

Phenylpropanoid and flavonoids

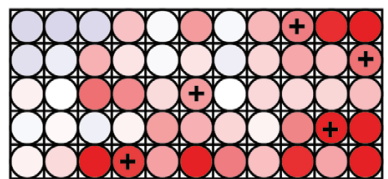

AT2G37040/AtPAL AT2G30490/AtC4H AT3G21240/At4CL2 AT5G13930/AtCHS AT2G43570/AtCHI

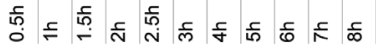

E Indole glucosinolates (GLS)

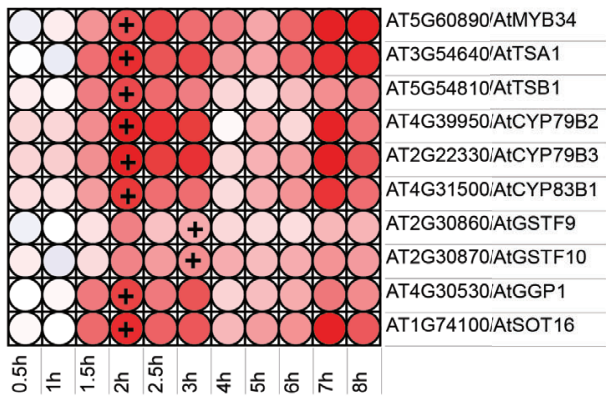

$\mathbf{F}$

G Aliphatic glucosinolates (GLS)

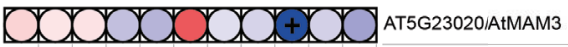

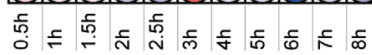
Indirect/GLVs pathway

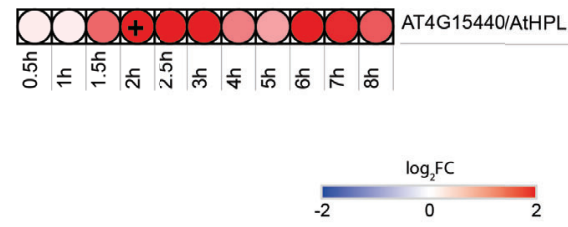

Figure S2. Expression pattern of Arabidopsis genes involved in biosynthesis of hormonal, secondary metabolites and indirect defences. (A) JA pathway, (B) ET pathway, (C) SA pathway, (D) phenylpropanoid and flavonoid pathways, (E) Indole glucosinolates (GLS), (F) Aliphatic glucosinolates (GLS), (G) Green-leaf volatiles (GLVs). '+' sign indicates significant $(P<0.01)$ first time of differential expression (ftode) of each gene. Based on data from Steenbergen et al. (2019). 



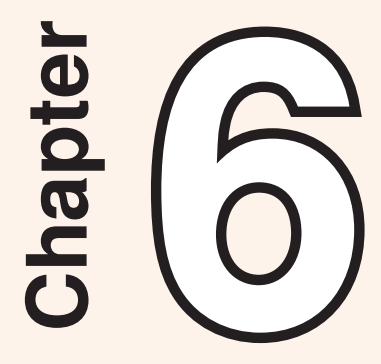

General discussion 



\section{Introduction}

Due to their sessile nature, plants constantly face attack from mobile herbivorous insects. In comparison to other biotic stresses, insect herbivores are the most species-rich group of plant attackers (Schoonhoven et al., 2005). To defend themselves, plants have evolved constitutive (always present) and induced defences (elicited by attack). Upon herbivore recognition, a plant induces defence mechanisms by activating signal transduction pathways that initiate the transcriptional responses. Subsequently, the transcriptional responses are dynamically reprogrammed resulting in the dynamic activation or deactivation of several biological processes (Windram et al., 2012; Hickman et al., 2017; Sarde et al., 2019; Steenbergen et al., 2019). This reorganization of transcriptional and biological processes causes extensive reprogramming of plant phenotype (Stam et al., 2014), that impacts the ecology of plants during the season (Poelman et al., 2010) or over seasons (Stam et al., 2018). Thus, unravelling transcriptional responses of plants in response to insect herbivory can shed light on the mechanisms underlying the dynamics of plant phenotype expression. In this thesis I have focussed on cell-content feeding thrips as insect herbivores. To unravel the details of thrips-inducible defence mechanisms, in this project I focused on elucidating the underlying mechanisms and temporal transcriptional responses of plants to thrips feeding.

To capture transcriptional responses of plants to insect herbivory, microarray and RNA-Seq analyses are extensively used. Nowadays, due to the decrease in the price of sequencing, the RNA-Seq technique is gaining popularity over microarray analysis (Heidel-Fischer et al., 2014). Moreover, RNA-Seq has several advantages over microarray analysis, such as 1) no background information on the genome is needed, 2) it offers high depth coverage in sequencing, 3) less complex normalization methods, 4) high accuracy and 5) detection of gene-splicing variants (Wang et al., 2009; Ozsolak \& Milos, 2011; Van Verk et al., 2013). A growing body of studies have analysed transcriptomic responses of plants against different insect herbivores (De Vos et al., 2005; Ehlting et al., 2008; Bidart-Bouzat \& Kliebenstein, 2011; Zhang et al., 2013; Appel et al., 2014; Coolen et al., 2016; Davila Olivas et al., 2016; Diaz-Riquelme et al., 2016; Kroes et al., 2017; Broekgaarden et al., 2018; Tu et al., 2018). These studies were performed for a limited number of time points since the start of herbivory, because they aimed mainly at elucidating the overall transcriptional responses and associated secondary metabolites, thus providing a low-resolution temporal representation of plant transcriptomic responses. To gain more insight into how plants temporally reprogram their transcriptome, performing high-density time-series experiments of transcriptional responses to insect herbivory is crucial. 
This project was part of a research programme which aimed to elucidate underlying genetic mechanisms of responses of sweet pepper to Western flower thrips (WFT) feeding and dynamics of transcriptional responses of Arabidopsis and sweet pepper to WFT feeding and white cabbage to onion thrips feeding. Several approaches, such as genomics, phylogenetics, high-density transcriptomics and behavioural studies were used in this thesis. To gain a high-resolution portrait of sweet pepper and white cabbage transcriptomic responses to thrips feeding, a state-of-the-art next generation transcriptomics/bioinformatics approach was implemented.

\section{Prominent role of LOX-mediated JA pathway in defence response against thrips}

In response to insect herbivory, plants activate phytohormonal pathways that regulate a suite of responses, including the biosynthesis of secondary metabolites and defence-related proteins. Based on the feeding mode of insects, plants trigger different phytohormonal pathways, such as the jasmonic acid (JA), ethylene (ET) and salicylic acid (SA) pathways, that regulate specific defences against different types of insect herbivory (Pieterse et al., 2009; Verhage et al., 2010; Pieterse et al., 2012; Stam et al., 2014). For example, responses to chewing insects like caterpillars (Reymond et al., 2004; De Vos et al., 2005) and cell-content feeding insects like thrips (Abe et al., 2008; Abe et al., 2009; Steenbergen et al., 2018) are mediated by the JA pathway, while responses to phloem-feeding insects like aphids and whiteflies are mediated by the SA pathway (Zhu-Salzman et al., 2004; Walling, 2008; Pieterse et al., 2012; Tzin et al., 2015; Broekgaarden et al., 2018).

JA biosynthesis occurs via several enzymatic steps, among which oxygenation of linolenic acid is the critical step to initiate the biosynthesis of oxylipins, such as jasmonates and green leaf volatiles (GLVs) that are known to be involved in defence responses against insect feeding (Brash, 1999; Feussner \& Wasternack, 2002; Allmann et al., 2010; Yan et al., 2013; Losvik et al., 2017). The lipoxygenase (LOX) gene family mediates several biological processes, such as fruit ripening, tuber development, seed germination and plant defences (Brash, 1999; Feussner \& Wasternack, 2002). By oxygenating linolenic acid (Kolomiets et al., 2001; Bailly et al., 2002; Feussner \& Wasternack, 2002; Kessler, 2004; Barry \& Giovannoni, 2007; Yan et al., 2013), plants activate JA-regulated defences in response to insect feeding. Information on this gene family has been reported for several plant species, such as Arabidopsis (Umate, 2011), tomato, kiwifruit (Zhang et al., 2006), olive (Padilla et al., 2009, 2012), melon (Zhang et al., 2014), cucumber (Liu et al., 2011) and grapevine (Podolyan et al., 2010). In chapter 2, the lipoxygenase (LOX) gene family from pepper (Capsicum annuum) has been identified and classified. In addition, based on in-depth in-silico and expression analysis, the functions of identified pepper LOXs were predicted. 
From the reported multiple LOXs in different plant species, usually one 13-LOX is involved in induced resistance of plants to insect herbivory via biosynthesis of JA and thus activating the JA signalling pathway. Several plant species show reduced resistance to insects, when the expression of that specific 13-LOX was disrupted; for example, AtLOX2 in Arabidopsis (Bell et al., 1995), SILOXD (TomLOXD) in tomato (Yan et al., 2013), NaLOX3 in tobacco (Halitschke \& Baldwin, 2003; Kessler, 2004) and StLOXH3 in potato (Royo et al., 1996). In chapter 3, through in-silico analysis, I narrowed down the LOX genes to a specific 13-LOX gene (CaLOX2) in pepper, and experimentally characterized its functional role in the JA-biosynthetic pathway, by silencing it through the Virus-Induced Gene Silencing (VIGs) technique. I subsequently showed that knocking down CaLOX2 made the plant more susceptible to WFT. Data presented in chapters 4 and 5 , on the induction of the whole JA cascade including 13-LOXs in sweet pepper and white cabbage upon WFT and onion thrips feeding, respectively, consolidates the prominent role of lipoxygenase-mediated JA pathway in the response of different plant species to thrips feeding.

\section{Extensive transcriptional reprogramming of host plants upon thrips feeding}

One of the global quantifiable measures of plant responses to insect herbivory is to capture the set of differentially expressed genes (Heidel-Fischer et al., 2014). Plants extensively reprogram their transcriptome with time in response to several biotic and abiotic stresses (Breeze et al., 2011; Windram et al., 2012; Hickman et al., 2017). So far, several studies have captured transcriptomic responses of plants to insect herbivory limited to one or two time points (De Vos et al., 2005; Ehlting et al., 2008; Bidart-Bouzat \& Kliebenstein, 2011; Zhang et al., 2013; Appel et al., 2014; Diaz-Riquelme et al., 2016; Kroes et al., 2017; Broekgaarden et al., 2018; Tu et al., 2018), with a focus on the identification of the differentially expressed genes and their involvement in the biosynthesis of different metabolites, but not the dynamics of the transcriptional reprogramming. In the studies presented in chapters 4 and 5, I unravelled the temporally differential expression of large numbers of genes in sweet pepper and white cabbage plants upon WFT and onion thrips feeding, respectively. A similar pattern of transcriptional reprogramming was observed in Arabidopsis in response to WFT feeding (Steenbergen et al., 2019). The diversity of temporal expression patterns of numerous genes in all three plants is an indication of the extensive transcriptional reprogramming that occurs in plants upon thrips feeding.

Speed, intensity and complexity of transcriptional response is plant- and stress-specific

Transcriptional reprogramming forms the basis of plant response to insect herbivory (Heidel-Fischer et al., 2014). Apart from overall identifying differentially expressed genes in a plant's transcriptomic response, the dynamics of transcriptional respons- 
es can also be assessed in several other ways, such as the speed, intensity and complexity of the response. The speed of transcriptional response can be measured based on the number of genes showing differential expression at different time points since the onset of the stress, whereas intensity and complexity can be determined by the overall number of differentially expressed genes (DEGs) and the number of expression patterns observed among all DEGs, respectively. Although several studies have performed transcriptomic studies in response to insect herbivory, not much is revealed about temporal transcriptional reprogramming as the studies were performed for only a few time points (De Vos et al., 2005; Ehlting et al., 2008; Bidart-Bouzat \& Kliebenstein, 2011; Zhang et al., 2013; Appel et al., 2014; Diaz-Riquelme et al., 2016; Kroes et al., 2017; Broekgaarden et al., 2018; Tu et al., 2018). To our knowledge, few studies have performed high-density time-series experiments to capture the temporal transcriptional reprogramming of plants against biotic and abiotic stresses. For example, leaf senescence in Arabidopsis (22 time points over a period of 39 days) (Breeze et al., 2011), response of Arabidopsis to Botrytis cinerea infection (24 time points over a period of 48 hours) (Windram et al., 2012) and Arabidopsis response upon exogenous JA application (15 time points over a period of 16 hours) (Hickman et al., 2017) have been studied.

In chapters 4 and 5, I investigated the differences in transcriptional responses between WFT-infested Arabidopsis (Steenbergen et al., 2019) and sweet pepper as well as onion-thrips-infested white cabbage plants. In these studies, I observed that the transcriptional response of white cabbage plants is characterized by a higher speed, intensity and complexity compared to the response of Arabidopsis and sweet pepper plants to thrips feeding. The transcriptional response of Arabidopsis to $B$. cinerea infection showed a higher magnitude (9838 DEGs representing ca $30 \%$ of the CATMA v3 microarray) and complexity (44 gene clusters) in transcriptional response, but a slow speed of response (Windram et al., 2012). Similar observations (6323 DEGs representing ca $22 \%$ of the CATMA v3 microarray and 48 clusters) were reported during leaf senescence of Arabidopsis (Breeze et al., 2011). Moreover, upon exogenous JA application to Arabidopsis, the transcriptional response was relatively rapid, but less intense (3611 DEGs representing ca $10 \%$ of the Arabidopsis genome (TAIR version 10) ) and complex (27 clusters) (Hickman et al., 2017) than for Arabidopsis in response to thrips feeding. The high magnitude and complexity of the transcriptional response of Arabidopsis to leaf senescence and $B$. cinerea infection could be due to the slow speed of these stresses. In contrast, exogenous JA application and thrips feeding, both relatively fast inducers, show relatively rapid and less intense transcriptional responses in plants. Nevertheless, the response of white cabbage plants against onion thrips was relatively rapid, intense and complex compared to the responses of Arabidopsis and sweet pepper to WFT. Taken together, 
comparative investigations of the speed, intensity and complexity of transcriptional responses will be interesting to gain understanding of the repertoire of plant responses to environmental stresses.

\section{Majority of transcription factors activated by JA are plant and stress-specific}

In response to different biotic and abiotic stresses, plants activate signal-transduction pathways that regulate defence responses. This includes induction of transcription factors (TFs), secondary metabolites and defence-related proteins. Phytohormones such as JA, SA and ET are central players in modulating defence responses against insect herbivory and pathogens (De Vos et al., 2005; Verhage et al., 2010; Erb et al., 2012; Pieterse et al., 2012; Stam et al., 2014), whereas abscisic acid is important in responses to abiotic stresses such as heat, drought and cold (Yamaguchi-Shinozaki \& Shinozaki, 2006; Kilian et al., 2007; Huang et al., 2008).

In chapters 4 and 5, I document conservation and specificities of TF families in Arabidopsis (Steenbergen et al., 2019) and sweet pepper (chapter 4) induced upon WFT feeding and white cabbage (chapter 5) induced upon onion thrips feeding. We found TF families such as bHLH, MYB and WRKY being overrepresented in all three plant species, with several others TF families specifically induced in each plant species. This suggests that, although JA is a prominent phytohormone regulating defence responses against thrips feeding, the majority of TF families activated by JA are different in Arabidopsis, sweet pepper (chapter 4) and white cabbage plants, regulating different defence mechanisms. Furthermore, in Arabidopsis, few studies showed significant induction of certain TF families in response to leaf senescence (Breeze et al., 2011), B. cinerea infection (Windram et al., 2012) and exogenous JA application (Hickman et al., 2017). Leaf senescence is known to activate several stress-related phytohormones, such as JA, SA and ABA (Weaver et al., 1998; Morris et al., 2000; He et al., 2002) whereas, $B$. cinerea activates the JA/ET signalling pathway (Thomma et al., 1998; Rowe et al., 2010). Surprisingly, more common TF families were induced in leaf senescence and $B$. cinerea stresses (WRKY, NAC, AP2-EREBP, and $\mathrm{C} 3 \mathrm{H}$ ) compared to exogenous JA application (bHLH, ERF, and MYB). In contrast, TF families induced upon exogenous application of JA are also induced in Arabidopsis (bHLH and MYB) (Steenbergen et al., 2019), sweet pepper (bHLH, ERF, and MYB) and white cabbage plants (bHLH, ERF, and MYB). Taken together, although JA is a central player in regulating transcriptional responses against $B$. cinerea, thrips feeding and leaf senescence, apart from a few TF families, the majority of TF families are induced in a plant or stress specific manner suggesting JA activates different TF families in different plant species to regulate specific defence responses. 


\section{Selection of time points for assessing transcriptional response of plants to her- bivore feeding}

In the past decade, considerable progress has been made to quantify plant transcriptional responses to feeding by herbivorous insects. In plants, the field of transcriptomics has made much progress with advanced technologies such as whole-genome microarray or RNA-Seq, since the early use of a microarray which elucidated differential transcription of 45 transcripts in Arabidopsis (Schena et al., 1995). Nowadays, due to low costs of next generation sequencing technologies (RNA-Seq), transcriptional profiling of non-model plant species can be carried out for responses to any insect species. Several studies have captured transcriptional responses of plants against different insect herbivory, but the selection of time points seems to have usually been done without extensive knowledge of temporal patterns in transcriptional responses. This random selection of time points may lead to missing a significant number of transcripts or missing the temporal dynamics of the transcriptional response, which may fluctuate especially at the onset of the plant's response. Therefore, to enhance the insight into the transcriptional responses of plants, it is not only important to make a high-density temporal assessment, but also to select the most relevant time points. Selection of the most relevant time points can help to capture the maximum transcriptional response in terms of number of differentially expressed genes as well as the dynamics of the complex response. In chapters 3 and 4 , due to monetary constraints we employed a strategy to select the most representative time points from a total of 13 time points within a period of the initial 9 hours of thrips herbivory. Based on initial sequencing of one replicate from 12 time points $(0.5,1.0,1.5,2.0,2.5,3.0$, 4.0, 5.0, 6.0, 7.0, 8.0, 9.0 hours of infestation) and subsequent in-silico analysis, six time points (excluding the $0 \mathrm{~h}$ time point) were selected and finalised to capture the transcriptomes of sweet pepper and white cabbage. Therefore, to capture maximum transcriptional responses of plants against insect herbivory, implementation of selection of time points in an experimental setup is of utmost important.

\section{Future perspectives}

Thrips are serious pests on various ornamental and food crops (Steenbergen et al., 2018), significantly reducing yield. There is considerable literature on induced defences in response to chewing and phloem-feeding insects compared to cell-content feeders. Likewise, several $R$-genes were successfully identified in different plants mostly against phloem feeders (Broekgaarden et al., 2011). High-resolution transcriptional data presented in this thesis can be explored to build gene-regulatory networks (GRNs), to select potential regulators (TFs) of or candidate genes providing resistance against thrips feeding, that can be further tested for functional analysis 
through overexpression or silencing. Those genes that are involved in the expression of the relevant phenotype can be used by breeders to develop thrips-resistant varieties. In addition to feeding damage, thrips also inflict indirect damage to plants by transmitting tospoviruses (Steenbergen et al., 2018). Thrips feeding elicits especially JA-regulated defences (Li et al., 2002; Abe et al., 2008; Abe et al., 2009; Sarde et al., 2018) (chapter 4 and 5), whereas, contrastingly, TSWV elicits SA-regulated defences (Abe et al., 2012). The antagonistic relation between the JA and SA pathways is well-known (Pieterse et al., 2012). Therefore, it would be interesting to compare the thrips-induced transcriptional response to the transcriptional response induced by viruliferous thrips. This can lead to understanding of the dynamics of transcriptomic response under more relevant conditions that can be a first step towards thrips and tospovirus-resistant crop varieties.

Furthermore, in the natural environment, plants encounter different biotic and abiotic stresses that occur in sequence or simultaneously. Few studies have investigated plant responses to combination of insect herbivory are different from plant responses to single insect herbivory (Pieterse \& Dicke, 2007; Dicke et al., 2009; Utsumi et al., 2010). Recent studies reported differences in phenotypic and transcriptomic plant responses to combined and single biotic and abiotic stresses (Coolen et al., 2016; Davila Olivas et al., 2016). Therefore, to understand and develop crop varieties possessing resistance to several stresses, studying transcriptional responses to combinations of stresses is important. Moreover, recent evidence shows how plant ontogeny influences the resistance level of plants, and especially the transition from vegetative stage to the flowering stage may alter plant resistance responses (Boege \& Marquis, 2005; Barton \& Koricheva, 2010; Diezel et al., 2011). It will be interesting to compare the whole-genome transcriptional responses of plants to thrips feeding in the vegetative and flowering stages. This may yield insights into the switch that occurs in plant defence mechanisms from vegetative to flowering stage including core TFs, and specialized metabolites.

\section{Conclusion}

In this thesis, I have elucidated underlying genetic mechanisms of sweet pepper in response to WFT feeding. By identifying and functionally characterizing the role of CaLOX2, I consolidated the importance of JA-regulated defences against thrips in sweet pepper. Furthermore, through high-density time-series RNA-Seq analysis of leaf tissue at 12 time points within the first 9 hours, I captured the dynamics of the early transcriptional response of sweet pepper and white cabbage plants to WFT and onion thrips feeding, respectively. Overall, the data represent a conservation of induction of JA, ET and phenylpropanoid and flavonoid pathways in sweet pepper and 
white cabbage. Furthermore, the genes involved in the biosynthesis of indolic glucosinolates were induced in white cabbage, whereas genes involved in biosynthesis of isoprenoids (terpenoids) were induced in sweet pepper. Comparative transcriptomics of the WFT-induced response of Arabidopsis and sweet pepper and the onion-thrips induced response of white cabbage plants suggests that the majority of transcriptomic responses against thrips are system-specific. Moreover, in comparison to Arabidopsis and sweet pepper, the transcriptomic response of white cabbage is more rapid and complex. This thesis provides a first impression of the complexity of early molecular aspects of thrips-plant interactions. The information generated will help to understand how plants defend themselves against these cell-content feeding insect herbivores.

\section{Acknowledgements}

I express my gratitude to Marcel Dicke for his constructive comments on an earlier version of this chapter. This work is part of STW perspective programme Green Defences Against Pests (GAP) that is (partly) financed by the Netherlands Organisation for Scientific Research (NWO, grant no. 13555).

\section{References}

Abe H, Ohnishi J, Narusaka M, Seo S, Narusaka Y, Tsuda S, Kobayashi M. 2008. Function of jasmonate in response and tolerance of Arabidopsis to thrip feeding. Plant Cell Physiology 49(1): 68-80.

Abe H, Shimoda T, Ohnishi J, Kugimiya S, Narusaka M, Seo S, Narusaka Y, Tsuda S, Kobayashi M. 2009. Jasmonate-dependent plant defense restricts thrips performance and preference. BMC Plant Biology 9: 97.

Abe H, Tomitaka Y, Shimoda T, Seo S, Sakurai T, Kugimiya S, Tsuda S, Kobayashi M. 2012. Antagonistic plant defense system regulated by phytohormones assists interactions among vector insect, Thrips and a Tospovirus. Plant and Cell Physiology 53(1): 204-212.

Allmann S, Halitschke R, Schuurink RC, Baldwin IT. 2010. Oxylipin channelling in Nicotiana attenuata: lipoxygenase 2 supplies substrates for green leaf volatile production. Plant Cell and Environment 33(12): 2028-2040.

Appel HM, Fescemyer H, Ehlting J, Weston D, Rehrig E, Joshi T, Xu D, Bohlmann J, Schultz J. 2014. Transcriptional responses of Arabidopsis thaliana to chewing and sucking insect herbivores. Frontiers in Plant Science 5: 565.

Bailly C, Bogatek-Leszczynska R, Come D, Corbineau F. 2002. Changes in activities of antioxidant enzymes and lipoxygenase during growth of sunflower seedlings from seeds of different vigour. Seed Science Research 12(1): 47-55.

Barry CS, Giovannoni JJ. 2007. Ethylene and fruit ripening. Journal of Plant Growth Regulation 26(2): 143-159.

Barton KE, Koricheva J. 2010. The ontogeny of plant defense and herbivory: Characterizing general patterns using meta-analysis. American Naturalist 175(4): 481-493.

Bell E, Creelman RA, Mullet JE. 1995. A chloroplast lipoxygenase is required for wound-induced jasmonic acid accumulation in Arabidopsis. Proceedings of the National Academy 
of Sciences of the United States of America 92(19): 8675-8679.

Bidart-Bouzat MG, Kliebenstein D. 2011. An ecological genomic approach challenging the paradigm of differential plant responses to specialist versus generalist insect herbivores. Oecologia 167(3): 677-689.

Boege K, Marquis RJ. 2005. Facing herbivory as you grow up: the ontogeny of resistance in plants. Trends in Ecology \& Evolution 20(8): 441-448.

Brash AR. 1999. Lipoxygenases: Occurrence, functions, catalysis, and acquisition of substrate. Journal of Biological Chemistry 274(34): 23679-23682.

Breeze E, Harrison E, McHattie S, Hughes L, Hickman R, Hill C, Kiddle S, Kim YS, Penfold CA, Jenkins D, Zhang CJ, Morris K, Jenner C, Jackson S, Thomas B, Tabrett A, Legaie R, Moore JD, Wild DL, Ott S, Rand D, Beynon J, Denby K, Mead A, Buchanan-Wollaston V. 2011. High-resolution temporal profiling of transcripts during Arabidopsis leaf senescence reveals a distinct chronology of processes and regulation. Plant Cell 23(3): 873-894.

Broekgaarden C, Pelgrom KTB, Bucher J, van Dam NM, Grosser K, Pieterse CMJ, van Kaauwen M, Steenhuis G, Voorrips RE, de Vos M, Vosman B, Worrich A, van Wees SCM. 2018. Combining QTL mapping with transcriptome and metabolome profiling reveals a possible role for $A B A$ signaling in resistance against the cabbage whitefly in cabbage. PLoS One 13(11): e0206103.

Broekgaarden C, Snoeren TAL, Dicke M, Vosman B. 2011. Exploiting natural variation to identify insect-resistance genes. Plant Biotechnology Journal 9(8): 819-825.

Coolen S, Proietti S, Hickman R, Davila Olivas NH, Huang PP, Van Verk MC, Van Pelt JA, Wittenberg AHJ, De Vos M, Prins M, Van Loon JJA, Aarts MGM, Dicke M, Pieterse CMJ, Van Wees SCM. 2016. Transcriptome dynamics of Arabidopsis during sequential biotic and abiotic stresses. Plant Journal 86(3): 249-267.

Davila Olivas NH, Coolen S, Huang PP, Severing E, van Verk MC, Hickman R, Wittenberg AHJ, de Vos M, Prins M, van Loon JJA, Aarts MGM, van Wees SCM, Pieterse CMJ, Dicke M. 2016. Effect of prior drought and pathogen stress on Arabidopsis transcriptome changes to caterpillar herbivory. New Phytologist 210(4): 1344-1356.

De Vos M, Van Oosten VR, Van Poecke RMP, Van Pelt JA, Pozo MJ, Mueller MJ, Buchala AJ, Metraux JP, Van Loon LC, Dicke M, Pieterse CMJ. 2005. Signal signature and transcriptome changes of Arabidopsis during pathogen and insect attack. Molecular Plant-Microbe Interactions 18(9): 923-937.

Diaz-Riquelme J, Zhurov V, Rioja C, Perez-Moreno I, Torres-Perez R, Grimplet J, Carbonell-Bejerano P, Bajda S, Van Leeuwen T, Martinez-Zapater JM, Grbic M, Grbic V. 2016. Comparative genome-wide transcriptome analysis of Vitis vinifera responses to adapted and non-adapted strains of two-spotted spider mite, Tetranyhus urticae. BMC Genomics 17: 74.

Dicke M, van Loon JJA, Soler R. 2009. Chemical complexity of volatiles from plants induced by multiple attack. Nature Chemical Biology 5(5): 317-324.

Diezel C, Allmann S, Baldwin IT. 2011. Mechanisms of optimal defense patterns in Nicotiana attenuata: Flowering attenuates berbivory-elicited ethylene and jasmonate signaling. Journal of Integrative Plant Biology 53(12): 971-983.

Ehlting J, Chowrira SG, Mattheus N, Aeschliman DS, Arimura GI, Bohlmann J. 2008. Comparative transcriptome analysis of Arabidopsis thaliana infested by diamond back moth (Plutella xylostella) larvae reveals signatures of stress response, secondary metabolism, and signalling. BMC Genomics 9: 154. 
Erb M, Meldau S, Howe GA. 2012. Role of phytohormones in insect-specific plant reactions. Trends in Plant Science 17(5): 250-259.

Feussner I, Wasternack C. 2002. The lipoxygenase pathway. Annual Review of Plant Biology 53: 275-297.

Halitschke R, Baldwin IT. 2003. Antisense LOX expression increases herbivore performance by decreasing defense responses and inhibiting growth-related transcriptional reorganization in Nicotiana attenuata. Plant Journal 36(6): 794-807.

He YH, Fukushige H, Hildebrand DF, Gan SS. 2002. Evidence supporting a role of jasmonic acid in Arabidopsis leaf senescence. Plant Physiology 128(3): 876-884.

Heidel-Fischer HM, Musser RO, Vogel H 2014. Plant transcriptomic responses to herbivory. Annual Plant Reviews: John Wiley \& Sons, Ltd, 155-196.

Hickman R, Van Verk MC, Van Dijken AJH, Mendes MP, Vroegop-Vos IA, Caarls L, Steenbergen M, Van der Nagel I, Wesselink GJ, Jironkin A, Talbot A, Rhodes J, De Vries M, Schuurink RC, Denby K, Pieterse CMJ, Wees SCM. 2017. Architecture and dynamics of the jasmonic acid gene regulatory network. Plant Cell 29(9): 2086-2105.

Huang DQ, Wu WR, Abrams SR, Cutler AJ. 2008. The relationship of drought-related gene expression in Arabidopsis thaliana to hormonal and environmental factors. Journal of Experimental Botany 59(11): 2991-3007.

Kessler A. 2004. Silencing the jasmonate cascade: Induced plant defenses and insect populations. Science 306: 2042-2042.

Kilian J, Whitehead D, Horak J, Wanke D, Weinl S, Batistic O, D’Angelo C, Bornberg-Bauer E, Kudla J, Harter K. 2007. The AtGenExpress global stress expression data set: protocols, evaluation and model data analysis of UV-B light, drought and cold stress responses. Plant Journal 50(2): 347-363.

Kolomiets MV, Hannapel DJ, Chen H, Tymeson M, Gladon RJ. 2001. Lipoxygenase is involved in the control of potato tuber development. Plant Cell 13(3): 613-626.

Kroes A, Broekgaarden C, Uribe MC, May S, van Loon JJA, Dicke M. 2017. Brevicoryne brassicae aphids interfere with transcriptome responses of Arabidopsis thaliana to feeding by Plutella xylostella caterpillars in a density-dependent manner. Oecologia 183(1): 107-120.

Li CY, Williams MM, Loh YT, Lee GI, Howe GA. 2002. Resistance of cultivated tomato to cell content-feeding herbivores is regulated by the octadecanoid-signaling pathway. Plant Physiology 130(1): 494-503.

Liu SQ, Liu XH, Jiang LW. 2011. Genome-wide identification, phylogeny and expression analysis of the lipoxygenase gene family in cucumber. Genetics and Molecular Research 10(4): 2613-2636.

Losvik A, Beste L, Glinwood R, Ivarson E, Stephens J, Zhu LH, Jonsson L. 2017. Overexpression and down-regulation of barley lipoxygenase LOX2.2 affects jasmonate-regulated genes and aphid fecundity. International Journal of Molecular Sciences 18(12): 2765; doi:2710.3390/ijms18122765.

Morris K, Mackerness SAH, Page T, John CF, Murphy AM, Carr JP, Buchanan-Wollaston V. 2000. Salicylic acid has a role in regulating gene expression during leaf senescence. Plant Journal 23(5): 677-685.

Ozsolak F, Milos PM. 2011. RNA sequencing: advances, challenges and opportunities. Nature Reviews Genetics 12(2): 87-98.

Padilla MN, Hernandez ML, Sanz C, Martinez-Rivas JM. 2009. Functional characterization of 
two 13-lipoxygenase genes from olive fruit in relation to the biosynthesis of volatile compounds of virgin olive oil. Journal of Agricultural and Food Chemistry 57(19): 9097-9107.

Padilla MN, Hernandez ML, Sanz C, Martinez-Rivas JM. 2012. Molecular cloning, functional characterization and transcriptional regulation of a 9-lipoxygenase gene from olive. Phytochemistry 74: 58-68.

Pieterse CMJ, Dicke M. 2007. Plant interactions with microbes and insects: from molecular mechanisms to ecology. Trends in Plant Science 12(12): 564-569.

Pieterse CMJ, Leon-Reyes A, Van der Ent S, Van Wees SCM. 2009. Networking by small-molecule hormones in plant immunity. Nature Chemical Biology 5(5): 308-316.

Pieterse CMJ, Van der Does D, Zamioudis C, Leon-Reyes A, Van Wees SCM. 2012. Hormonal modulation of plant immunity. Annual Review of Cell and Developmental Biology, Vol 28 28: 489-521.

Podolyan A, White J, Jordan B, Winefield C. 2010. Identification of the lipoxygenase gene family from Vitis vinifera and biochemical characterisation of two 13-lipoxygenases expressed in grape berries of Sauvignon Blanc. Functional Plant Biology 37(8): 767-784.

Poelman EH, Van Loon JJA, Van Dam NM, Vet LEM, Dicke M. 2010. Herbivore-induced plant responses in Brassica oleracea prevail over effects of constitutive resistance and result in enhanced herbivore attack. Ecological Entomology 35(2): 240-247.

Reymond P, Bodenhausen N, Van Poecke RM, Krishnamurthy V, Dicke M, Farmer EE. 2004. A conserved transcript pattern in response to a specialist and a generalist herbivore. Plant Cell 16(11): 3132-3147.

Rowe HC, Walley JW, Corwin J, Chan EKF, Dehesh K, Kliebenstein DJ. 2010. Deficiencies in jasmonate-mediated plant defense reveal quantitative variation in Botrytis cinerea pathogenesis. Plos Pathogens 6(4): e1000861.

Royo J, Vancanneyt G, Perez AG, Sanz C, Stormann K, Rosahl S, SanchezSerrano JJ. 1996. Characterization of three potato lipoxygenases with distinct enzymatic activities and different organ-specific and wound-regulated expression patterns. Journal of Biological Chemistry 271(35): 21012-21019.

Sarde S, Hickman R, Steenbergen M, Wiegers G, Pieterse CMJ, Van Wees SCM, Dicke M. 2019. Whole-genome transcriptional reprogramming of sweet pepper in response to Western flower thrips feeding. In prep.

Sarde SJ, Bouwmeester K, Venegas-Molina J, David A, Boland W, Dicke M. 2018. Involvement of sweet pepper CaLOX2 in jasmonate-dependent induced defence against Western flower thrips. Journal of Integrative Plant Biology: 10.1111/jipb.12742.

Schena M, Shalon D, Davis RW, Brown PO. 1995. Quantitative monitoring of gene-expression patterns with a complementary-DNA microarray. Science 270(5235): 467-470.

Schoonhoven LM, Van Loon JJA, Dicke M. 2005. Insect plant biology. Oxford University Press, Oxford: $421 \mathrm{pp}$.

Stam JM, Dicke M, Poelman EH. 2018. Order of herbivore arrival on wild cabbage populations influences subsequent arthropod community development. Oikos 127(10): 1482-1493.

Stam JM, Kroes A, Li YH, Gols R, van Loon JJA, Poelman EH, Dicke M. 2014. Plant interactions with multiple insect herbivores: From community to genes. Annual Review of Plant Biology 65: 689-713.

Steenbergen M, Abd-el-Haliem A, Bleeker P, Dicke M, Escobar-Bravo R, Cheng G, Haring MA, Kant MR, Kappers I, Klinkhamer PGL, Leiss KA, Legarrea S, Macel M, Mouden S, Pieterse CMJ, Sarde SJ, Schuurink RC, De Vos M, Van Wees SCM, Broekgaarden 
C. 2018. Thrips advisor: Exploiting thrips-induced defences to combat pests on crops. Journal of Experimental Botany 69(8): 1837-1848.

Steenbergen M, Hickman R, Sarde S, Dicke M, Pieterse CMJ, Van Wees SCM. 2019. High-resolution temporal transcriptomic dynamics of Arabidopsis thaliana in response to Western flower thrips feeding. In prep.

Thomma BPHJ, Eggermont K, Penninckx IAMA, Mauch-Mani B, Vogelsang R, Cammue BPA, Broekaert WF. 1998. Separate jasmonate-dependent and salicylate-dependent defense-response pathways in Arabidopsis are essential for resistance to distinct microbial pathogens. Proceedings of the National Academy of Sciences of the United States of America 95(25): 15107-15111.

Tu XB, Liu ZK, Zhang ZH. 2018. Comparative transcriptomic analysis of resistant and susceptible alfalfa cultivars (Medicago sativa L.) after thrips infestation. BMC Genomics 19: 10.1186/s12864-018-4495-2.

Tzin V, Fernandez-Pozo N, Richter A, Schmelz EA, Schoettner M, Schafer M, Ahern KR, Meihls LN, Kaur H, Huffaker A, Mori N, Degenhardt J, Mueller LA, Jander G. 2015. Dynamic maize responses to aphid feeding are revealed by a time series of transcriptomic and metabolomic assays. Plant Physiology 169(3): 1727-1743.

Umate P. 2011. Genome-wide analysis of lipoxygenase gene family in Arabidopsis and rice. Plant Signaling and Behavior 6(3): 335-338.

Utsumi S, Ando Y, Miki T. 2010. Linkages among trait-mediated indirect effects: a new framework for the indirect interaction web. Population Ecology 52(4): 485-497.

Van Verk MC, Hickman R, Pieterse CMJ, Van Wees SCM. 2013. RNA-Seq: revelation of the messengers. Trends in Plant Science 18(4): 175-179.

Verhage A, van Wees SCM, Pieterse CMJ. 2010. Plant immunity: It's the hormones talking, but what do they say? Plant Physiology 154(2): 536-540.

Walling LL. 2008. Avoiding effective defenses: Strategies employed by phloem-feeding insects. Plant Physiology 146(3): 859-866.

Wang Z, Gerstein M, Snyder M. 2009. RNA-Seq: a revolutionary tool for transcriptomics. Nature Reviews Genetics 10(1): 57-63.

Weaver LM, Gan SS, Quirino B, Amasino RM. 1998. A comparison of the expression patterns of several senescence-associated genes in response to stress and hormone treatment. Plant Molecular Biology 37(3): 455-469.

Windram O, Madhou P, McHattie S, Hill C, Hickman R, Cooke E, Jenkins DJ, Penfold CA, Baxter L, Breeze E, Kiddle SJ, Rhodes J, Atwell S, Kliebenstein DJ, Kim YS, Stegle O, Borgwardt K, Zhang CJ, Tabrett A, Legaie R, Moore J, Finkenstadt B, Wild DL, Mead A, Rand D, Beynon J, Ott S, Buchanan-Wollaston V, Denby KJ. 2012. Arabidopsis defense against Botrytis cinerea: Chronology and regulation deciphered by high-resolution temporal transcriptomic analysis. Plant Cell 24(9): 3530-3557.

Yamaguchi-Shinozaki K, Shinozaki K. 2006. Transcriptional regulatory networks in cellular responses and tolerance to dehydration and cold stresses. Annual Review of Plant Biology 57: 781-803.

Yan LH, Zhai QZ, Wei JN, Li SY, Wang B, Huang TT, Du MM, Sun JQ, Kang L, Li CB, Li CY. 2013. Role of Tomato lipoxygenase $D$ in wound-induced jasmonate biosynthesis and plant immunity to insect herbivores. PLoS Genetics 9(12): e1003964.

Zhang B, Chen KS, Bowen J, Allan A, Espley R, Karunairetnam S, Ferguson I. 2006. Differential expression within the LOX gene family in ripening kiwifruit. Journal of Experimental Botany 57(14): 3825-3836. 
Zhang C, Jin YZ, Liu JY, Tang YF, Cao SX, Qi HY. 2014. The phylogeny and expression profiles of the lipoxygenase ( $L O X$ ) family genes in the melon (Cucumis melo L.) genome. Scientia Horticulturae 170: 94-102.

Zhang PJ, Broekgaarden C, Zheng SJ, Snoeren TAL, van Loon JJA, Gols R, Dicke M. 2013. Jasmonate and ethylene signaling mediate whitefly-induced interference with indirect plant defense in Arabidopsis thaliana. New Phytologist 197(4): 1291-1299.

Zhu-Salzman K, Salzman RA, Ahn JE, Koiwa H. 2004. Transcriptional regulation of sorghum defense determinants against a phloem-feeding aphid. Plant Physiology 134(1): 420-431. 

Summary 

In nature, plants continuously face the attack from insect herbivores. To defend themselves, plants have evolved a plethora of direct and indirect defence mechanisms that are present either constitutively or induced upon insect herbivory. In response to insect herbivory, plants activate phytohormonal signal-transduction pathways, transcriptional responses and biological processes. These changes in transcriptional and biological processes level influence the plant's phenotype and consequently plant ecology during the current season or over seasons. To gain more insight into the dynamics of plant phenotype, elucidating transcriptomic responses to insect herbivory is critical. Nowadays, to capture transcriptional responses against insect herbivory, RNA-Seq technology is used extensively. Various studies have investigated whole-genome transcriptional responses of plants against different insect herbivores, but only few have reported on responses to cell-content feeding thrips. Thrips (Thysanoptera) are minute cell-content feeding insects and are serious pests on many commercial and ornamental plants. Thrips species such as western flower thrips (WFT) and onion thrips are among the most devastating pests on e.g. sweet pepper and white cabbage plants, respectively. Therefore, using a high-density time-series approach, the focus of this thesis was to investigate whole-genome transcriptional responses of sweet pepper and white cabbage plants upon WFT and onion thrips feeding, respectively, and the underlying genetic mechanisms. In addition, I focussed on one particular gene family, the lipoxygenases and a gene in this family that is involved in thrips-induced crop resistance.

Chapter 2 focused on the identification of the lipoxygenase gene family in pepper (Capsicum annuum). Lipoxygenases (LOXs) are non-heme, iron-containing dioxygenases involved in several developmental and defence-related plant processes, such as seed germination, fruit ripening, tuber development and JA-regulated plant defences. To identify this multi-gene family, several approaches were implemented, such as comparative genomics, sequence analysis, domain-scan analysis, phylogenetic analysis, homology modelling and transcriptional analysis. This approach resulted in the identification of a total of eight LOX genes in pepper classifying four LOXs (CaLOX1, CaLOX3, CaLOX4 and CaLOX5) as 9-LOXs and four (CaLOX2, CaLOX6, CaLOX7 and CaLOX8) as 13-LOXs. Furthermore, these approaches also showed high conservation of pivotal amino acids and dynamic expression profiles of 13-LOXs compared to 9-LOXs upon exogenous JA application and WFT feeding. From the results, the putative functions of two 13-LOXs, CaLOX6 and CaLOX7, in the biosynthesis of Green Leaf Volatiles (GLVs) were predicted.

Chapter 3 further narrowed down the LOX gene-family to one lipoxygenase (CaLOX2) gene through in-silico analysis and functionally characterized its involvement in jasmonate-dependent induced defence against WFT. Here, I implemented a multidisci- 
plinary approach which includes in-silico, transcriptional, behavioural and chemical analyses. The expression of JA-related marker genes (CaLOX2 and CaPIN II) was induced upon thrips feeding. Silencing of CaLOX2 in sweet pepper plants through virus-induced gene silencing (VIGS) significantly hampered the biosynthesis of the phytohormone JA and its derivatives. Subsequently, this resulted into reduced resistance and increased preference of thrips for these sweet pepper plants. Furthermore, thrips feeding ability, preference and population development were hampered upon exogenous JA application. Overall, this chapter shows that CaLOX2 is involved in JA-mediated plant resistance to thrips feeding.

In response to insect feeding, temporal transcriptional reprogramming forms the basis of dynamically changing plant phenotype. Therefore, in Chapter 4, I focused on elucidating the dynamics of transcriptional reprogramming of sweet pepper leaf tissue in response to WFT feeding. For this, leaf tissue of sweet pepper plants that were subjected to WFT infestation was harvested at 12 time points within the first 9 hours of WFT feeding for RNA-Seq analysis. Approximately 8.6\% (2060 up- and 1002 down-regulated) of the pepper genes were differentially expressed upon WFT feeding. Subsequent in-depth analysis of a selected set of 6 time points categorized the 3062 DEGs into 23 gene clusters (16 upregulated and 7 downregulated), each possessing a unique temporal expression pattern. Clusters of upregulated genes were associated with defence-related biological processes, whereas clusters of downregulated genes were associated with developmental processes. The transcription factor families ERF, MYB, NAC, bHLH and WRKY emerged as pivotal regulators in response to WFT feeding. The data show a chronological order in the activation of hormonal (JA, ET) and secondary metabolite (phenylpropanoids, flavonoids and terpenoids) pathways. Eventually, the comparative analysis of the WFT-induced transcriptional responses of Arabidopsis and sweet pepper plants to WFT feeding shows a conservation in the induction of the JA-pathway in both plants, whereas the majority of transcriptional responses are plant-specific. In addition, it also showed that relatively more similarities exist in upregulated genes compared to downregulated genes.

Chapter 5 focused on 1) elucidating whole-genome transcriptional reprogramming of white cabbage plants in response to onion thrips feeding and 2) comparative transcriptomics to disentangle similarities and differences in transcriptional responses between WFT-induced Arabidopsis and sweet pepper as well as onion-thrips-induced white cabbage. The data for all three plant species were collected through a similar approach. Approximately $9.7 \%$ of the white cabbage genes show differential expression within $8 \mathrm{~h}$ of onion thrips feeding. Forty-eight (32 upregulated and 16 downregulated) gene clusters with similar expression patterns were detected among the DEGs, with upregulated clusters associated with defence and downregulated clus- 
ters with development-related biological processes. Phytohormone-related processes (JA, ET and SA) and secondary metabolite (phenylpropanoids, flavonoids, green-leaf volatiles and indolic glucosinolates) biosynthesis genes were induced, whereas aliphatic glucosinolate biosynthetic genes were suppressed. Comparative analysis of the transcriptional responses of Arabidopsis and sweet pepper to WFT and of white cabbage to onion thrips showed 1) conservation of the JA biosynthesis and signalling pathways, 2) conservation of involvement of TF families, such as MYB, bHLH and WRKY in regulating responses, 3) that the majority of the transcriptional responses to thrips are system-specific, 4) that genes involved in indole glucosinolate biosynthesis are upregulated, whereas genes involved in aliphatic glucosinolate biosynthesis are downregulated in both brassicaceous plants Arabidopsis and white cabbage, 5) that the white-cabbage transcriptomic response to onion thrips is relatively rapid and complex compared to the WFT-induced Arabidopsis and sweet pepper transcriptomic responses.

Finally, in Chapter 6, I discuss the dynamics of plant transcriptional responses to several stresses. This includes the importance of the LOX-mediated JA pathway against thrips feeding and several aspects of plants transcriptional reprogramming to thrips and other stresses. The advantages of the selection of time points in a high-density time series approach, as implemented in this project are discussed. Furthermore, I make recommendations to enhance our knowledge of induced defences in plants. In conclusion, this thesis highlights the intensity and complexity of plant transcriptional responses to thrips feeding through a comparative approach, while focussing on the details of one important gene involved in induced defence against thrips. 



\section{Acknowledgements}

Time flies and the four year adventure of my PhD has come to an end. This PhD adventure was like a roller-coaster ride full of learning, challenges, excitement, frustrations and a combination of successful and failed experiments. This journey wouldn't be accomplished without the support of amazing people around me. For that, I would like express my gratitude to all the people of the Laboratory of Entomology (WUR), the Plant-Microbe Interactions group (Utrecht University) and the Laboratory of Phytopathology (WUR).

I would like to begin my acknowledgement note by offering my sincerest gratitude to my supervisor, Prof. Dr. Marcel Dicke. Dear Marcel, it was an honour and real privilege for me to pursue my $\mathrm{PhD}$ under your guidance. I appreciate your patient supervision, great support and encouragements provided throughout my PhD journey. You provided me with immense freedom in all aspects of the PhD that helped me to explore my own research ideas. Many thanks for making time within your busy schedule and providing urgent feedback on my PhD thesis chapters. I have learned a lot from you and I immensely admire your leadership, scientific knowledge and human qualities that will help me to grow not only professionally but also personally.

This PhD project was the part of TTW (former STW) consortium entitled "Green defence Against Pests (GAP)", which involved collaboration with several people from different universities and companies. My acknowledgements to core collaborators: Prof. Dr. Corné Pieterse, Dr. Saskia van Wees, Dr. Richard Hickman, Merel Steenbergen and Dr. Colette Broekgaarden. Many thanks to Corné Pieterse and Saskia van Wees for your constant support, constructive suggestions and availability that were the basis to accomplish this PhD project. I am extremely grateful to Richard for your guidance, input and lively discussions on transcriptomics data. I enjoyed collaborating with you and learnt a lot from you. Merel, thanks for all the help you provided during the project. Many thanks to Colette Broekgaarden for suggesting me to perform thrips treatment in one of my experiments. I also appreciate all my collaborators and co-authors for their help. I want to offer my sincere gratitude to Dr. Klaas Bouwmeester for guiding me on performing VIGs experiments. I enjoyed collaborating with you and without your input, one of my chapters would not be possible. I am thankful to Anja David and Prof. dr. Wilhelm Boland (Max Planck Institute for Chemical Ecology, Jena) for phytohormone quantification for one of my chapters. Many thanks to Dr. Rob Schuurink, Dr. Ahmed Abd-El-Haliem and Dr. Pulu Sun for lively discussions during our GAP meetings. Ahmed Abd-El-Haliem, thanks a lot for giving me a direction on comparative genomics and always driving me back to Wageningen from GAP 
meetings. I would also appreciate the efforts of my Master's students John Venegas Molina, Kiros Afewerki and Rahima Remme, for their contributions to generating interesting results.

In my project, several companies participated through sharing their ideas, knowledge and resources. I am thankful to Dr. Martin De Vos and Dr. Alexander Wittenberg (Keygene N.V., Wageningen) for performing RNA sequencing in the project. Many thanks to other representatives: Dr. Karin Posthuma (Enza Zaden), Bart André (Enza Zaden), Dr. Adriaan Verhage (Rijk Zwaan), Jan Bruin (Syngenta), Dr. Jedeliza Ferrater (East-West Seed) and Dr. Conrado Balatero (East-West Seed) to share ideas and in-kind contributions. To perform the experiments in this project, logistics of synchronized thrips L2 larvae was the most intricate phase. Many thanks to Gerrie Wiegers, Pauline van Haperen, Betty Henken and Dr. Gerben Messelink for help in managing the thrips culture for my experiments. Special thanks to Gerrie Wiegers and Betty Henken for always helping me out with the thrips culture and also providing me with space and resources to perform some of my experiments. I would also like to thank Unifarm people for taking care of my plants: Andre Maassen, Bertus vander Laan, Alex Super, Casper Pillen, Bert Essenstam, Sean Geurts, Harm Wiegersma and Gerrit Stunnenberg.

I wish to express my thanks to all the colleagues of Entomology for being so kind, hospitable and inspiring. Tim and Viola, thanks for all the help, hospitality and for being such an amazing couple. Viola, I appreciate you introducing Pooja to Wageningen. Kay, you are just amazing. Thanks for providing me urgent access to NIOO and for being helpful on all instances. Karol, thanks for being an such an awesome person and for organizing the Latin parties. Lucilleeeee ;), thanks for giving me some tips during the writing phase and I will definitely miss our conversations on social issues. Quint Rusman/Richman/Rudeman, there are so many alternatives to call you ;). Thanks man, for all the quint-sanity and hilarious conversations we had during this time. Antonino, I appreciate your help on statistical analysis. I also really enjoyed having conversations with you during the coffee breaks. Maite, what can I say, thanks for those hilarious conversations ;) and teaching me a bit of salsa ;). Alexandre, I really appreciate your social awareness. Thanks for those comprehensive discussions on social issues and for introducing me to tennis ;). Katherine, you are a true photocopy of your sister ;). Thanks for those delicious Colombian sweets. Patrick, thanks a lot for all the help in molecular biology laboratory. Yidong, I am extremely thankful for your help in the molecular biology lab and especially with graphic design. Peter, thanks for having the discussions on transcriptomics data. I would also like to thank all the colleagues who made time to attend my marriage: Kay, Lucille, Karol, Dani, Tim, Viola, Daan, Ilse, Quint and Jeroen. It was awesome to have you guys at the 
marriage. Quint, Daan and Bram, it was great to be part of the organizing committee of the $12^{\text {th }}$ Plant-Insect Interactions with you guys. Jessica, Yavanna, Yidong, Stijn, Maite, Jeroen and Julia, I enjoyed organising different events with you guys in the Ento party committee. Jessica, Stijn and Tim Stouten, it was fun to organize the Labuitje with you guys. Many thanks to Rieta, Léon, Jessica, Jhon, Merel, Jeroen, Kay, Maite, Yidong, Karol, Antonino, Afita, Tim, Keiko, Janneke and Rocky for helping me out with infestation and harvesting samples during my humongous time-series experiments. Angelique, many thanks for helping out with administrative work. I would also like to thank former Ento colleagues: Nelson, Dani, Manus, Karen, Ana, Nurmi and Enric for their warm welcome during start of my PhD.

Further, I would like to thank all my Indian friends to make this place my home: Gurnoor Singh, Sneha Gulati, Bandan Chakraborty, Manjunath Prasad, Trupti Sharma, Sidharam and Sunanda Pujari, Priyanka Singh, Satesh Gangarapu, Purva Kulkarni, Soumya Kanti Kar, Raka Choudhury and Suraj Jamge. Gurnoooooor bhai, thanks a lot for being such an amazing friend. Your jokes really made me laugh crazily. Sneha and Bandan, I really had quality time with you guys. I still remember, while I was sleeping in car, you guys drove me to Cologne instead of Aachen and made me believe it ;). Suraj, Manjunath, Priyanka, Satesh, Sidhu and Sunandaji, thanks for nice dinners and fun activities. I also would like to thank my cricketing mates who kept my cricketing hobby alive and refreshed me from stressful week days: Ameya Kale, Tanveer Bhatti, Mihris Naduthodi, Shimlal Ayilalath, Narendra Kata, Athul Sundaresan, Abhijeet Munde, Nitin Chandra, Parth Shah, Tarun Goli, Jonathan James, Parth Shah, Vineeth Venkatraman, Pavan Jakkamputi, Varun Madapally, Arun Balasubramaniam, Chitrateja Kothapeta and Vaibhav Mahilang.

To finish, I owe tons of thanks to my family for all their love and support. I am especially grateful to my mother, for supporting me in all of my pursuits and inspiring me to follow my dreams. I am privileged to be your son and I always knew that you believed in me and wanted the best out of me. Thanks to my sisters, Archana and Aparna and brother, Samadhan, for your support, encouragement and always standing beside me. Finally, I owe millions of thanks to my wife and my best friend, Pooja. It wouldn't be possible to finish the thesis without your support. Many thanks for showing support and trust in me during this challenging time. 


\section{Curriculum Vitae}

Sandeep J. Sarde was born on 27 September, 1986 in Mangrul, Maharashtra, India. Growing up with surroundings and family of farmers, his passion for agriculture has been extensively nurtured since childhood. After obtaining the Bachelor degree in Biotechnology with distinction, he pursued his Master's degree in Agrigenomics at the University of Kiel (CAU), Germany, with a focus on plant breeding, phytopathology and genomics in applied agriculture. He performed his Master's thesis entitled "Unravelling of the spli-

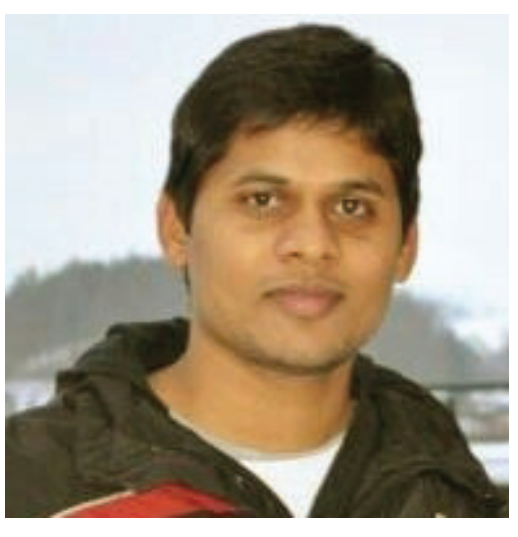
ceosomal proteins encoded by fungal genomes" by implementing the approach of comparative genomics under the supervision of Prof. Dr. rer. nat. Frank Kempken and Dr. Abhishek Kumar. During the Master's programme, he was also involved in volunteer projects with Dr. Abhishek Kumar to determine the evolutionary history and genetic variants of different serpins in the vertebrates, particularly involved in blood-related diseases.

In January 2015, he started his PhD project in the Laboratory of Entomology at Wageningen University, The Netherlands. The PhD program was part of the TTW (former STW) consortium entitled "Green defence Against Pests (GAP)". This GAP STW perspective program aimed to elucidate plant defence responses against cell-content feeding insects. The project was executed using multi-disciplinary approaches by the team effort of different Dutch universities and private agricultural companies such as, Rijk Zwaan, Enza Zaden, Syngenta, Keygene, East-West Seed and Bejo. In this consortium, Sandeep contributed to understanding of the dynamics of transcriptional responses of plants (sweet pepper, white cabbage and Arabidopsis thaliana) to thrips feeding and has presented his findings in this thesis. 



\section{Publication list}

\section{Published articles in a journal}

Sarde SJ, Kumar A, Remme RN, Dicke M. 2018. Genome-wide identification, classification and expression of lipoxygenase gene family in pepper. Plant Molecular Biology 98 (4-5): 375-387. (Chapter 2 in this thesis)

Sarde SJ, Bouwmeester K, Venegas-Molina J, David A, Boland W, Dicke M. 2018. Involvement of sweet pepper CaLOX2 in jasmonate-dependent induced defence against Western flower thrips. Journal of Integrative Plant Biology: 10.1111/ jipb.12742. (Chapter 3 in this thesis)

Sarde SJ, Kumar A, Kempken F. 2018. Spliceosomal proteins encoded by fungal genomes. Current Science 114(8): 1677-1686.

Vaello T, Sarde SJ, Marcos-Garcia MA, de Boer JG, Pineda A. 2018. Modulation of plant-mediated interactions between herbivores of different feeding guilds: Effects of parasitism and belowground interactions. Scientific Reports 8: 14424.

Steenbergen M, Abd-el-Haliem A, Bleeker P, Dicke M, Escobar-Bravo R, Cheng G, Haring MA, Kant MR, Kappers I, Klinkhamer PGL, Leiss KA, Legarrea S, Macel M, Mouden S, Pieterse CMJ, Sarde SJ, Schuurink RC, De Vos M, Van Wees SCM, Broekgaarden C. 2018. Thrips advisor: exploiting thrips-induced defences to combat pests on crops. Journal of Experimental Botany 69(8): 1837-1848.

Kumar A, Bhandari A, Sarde SJ, Goswami C. 2017. Ancestry \& molecular evolutionary analyses of heat shock protein $47 \mathrm{kDa}$ (HSP47/SERPINH1). Scientific Reports 7: 10394.

Kumar A, Bhandari A, Sarde SJ, Muppavarapu S, Tandon R. 2016. Data on the evolutionary history of the $\mathrm{V}(\mathrm{D}) \mathrm{J}$ recombination-activating protein 1 - RAG1 coupled with sequence and variant analyses. Data in Brief 8: 87-92.

Kumar A, Bhandari A, Sarde SJ, Muppavarapu S, Tandon R. 2015. Understanding $\mathrm{V}(\mathrm{D}) \mathrm{J}$ recombination initiator RAG1 gene using molecular phylogenetic and genetic variant analyses and upgrading missense and non-coding variants of clinical importance. Biochemical and Biophysical Research Communications 462(4): 301-313.

Kumar A, Sarde SJ, Bhandari A. 2014. Revising angiotensinogen from phylogenetic and genetic variants perspectives. Biochemical and Biophysical Research Communications 446(2): 504-518. 
Kumar A, Bhandari A, Sarde SJ, Goswami C. 2014. Genetic variants and evolutionary analyses of heparin cofactor II. Immunobiology 219(9): 713-728.

Kumar A, Bhandari A, Sarde SJ, Goswami C. 2014. Molecular phylogeny of C1 inhibitor depicts two immunoglobulin-like domains fusion in fishes and ray-finned fishes specific intron insertion after separation from zebrafish. Biochemical and Biophysical Research Communications 450(1): 219-226.

Kumar A, Bhandari A, Sarde SJ, Goswami C. 2013. Sequence, phylogenetic and variant analyses of antithrombin III. Biochemical and Biophysical Research Communications 440(4): 714-724.

\section{Articles in preparation}

Sarde SJ, Hickman R, Steenbergen M, Wiegers G, Pieterse CMJ, Van Wees SCM, Dicke M. 2019. Whole-genome transcriptional reprogramming of sweet pepper in response to Western flower thrips feeding. Submitted. (Chapter 4 in this thesis)

Sarde SJ, Hickman R, Steenbergen M, Wiegers G, Pieterse CMJ, Van Wees SCM, Dicke M. Comparative high-density transcriptomics reveals rapid and complex rearrangement of white cabbage transcriptome in response to thrips feeding. In prep. (Chapter 5 in this thesis)

Steenbergen M, Hickman R, Sarde SJ, Dicke M, Pieterse CMJ, Van Wees SCM. High-resolution temporal transcriptomic dynamics of Arabidopsis thaliana in response to Western flower thrips feeding. In prep. 


\section{Education Statement of the Graduate School Experimental Plant Sciences}

Issued to: Sandeep Jayaji Sarde

Date: 20 June 2019

Group: Laboratory of Entomology

University: Wageningen University \& Research

1) Start-Up Phase

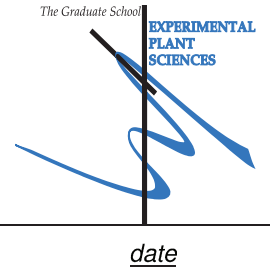

- First presentation of your project

Plants-thrips Interaction: uncovering dynamics of the plant defense signaling network to identify novel leads for maximizing crop protection

21 April , 2015

- Writing or rewriting a project proposal

Writing a review or book chapter

- MSc courses

\section{2) Scientific Exposure}

- EPS PhD student days

EPS PhD student days, Soest

EPS PhD student days, Soest

EPS PhD student days, Soest

- EPS theme symposia

EPS Theme 2 Symposium: Interactions between Plants and biotic agents, Utrecht EPS Theme 2 Symposium: Interactions between Plants and Biotic Agents, Leiden EPS Theme 1 Symposium: Developmental Biology of Plants, Leiden

EPS Theme 3 Symposium: Metabolism and Adaptation, Wageningen

- Lunteren Days and other national platforms

ALW meeting Experimental Plant Sciences, Lunteren

ALW meeting Experimental Plant Sciences, Lunteren

ALW meeting Experimental Plant Sciences, Lunteren

Annual meeting of Netherlands Entomological Society, Ede

Annual meeting of Netherlands Entomological Society, Ede

Green defence Against Pests (GAP) consortium meeting

Green defence Against Pests (GAP) consortium meeting

Green defence Against Pests (GAP) consortium meeting

Green defence Against Pests (GAP) consortium meeting

Green defence Against Pests (GAP) consortium meeting

Green defence Against Pests (GAP) consortium meeting

Green defence Against Pests (GAP) consortium meeting

Green defence Against Pests (GAP) consortium meeting

Green defence Against Pests (GAP) consortium meeting

- Seminars (series), workshops and symposia

EPS seminar by Florian Schiestl: Evolution of floral signals in plants: mechanisms and consequences

EPS seminar by Alain Goossens: How jasmonates provide the key to harness plant chemistry

EPS seminar by Mark Varrelmann: The rhizomania complex in sugar beet - virus variation and resistance breaking

EPS seminar by Martin Cann: The immune receptor Rx1 remodels chromatin and chromatin interactors in immunity

EPS seminar by Sanjay Kapoor: Regulators of Reproductive Development in Rice date

29 - 30 January, 2015

28 - 29 January, 2016

9-10 February, 2017

20 February, 2015

22 January, 2016

28 February, 2017

14 March, 2017

13 -14 April, 2015

11-12 April , 2016

10-11 April , 2017

15 December, 2017

14 December, 2018

21 April , 2015

25 September, 2015

6 April, 2016

11 October, 2016

18 May, 2017

27 November, 2017

13 March, 2018

21 September, 2018

29 November, 2018

12 March, 2015

8 December, 2015

18 April, 2017

11 July, 2017

29 August, 2017 
EPS seminar by U. Wyss: Highlights of hidden insect-worlds

EPS seminar by Asaf Levy: Bacteria and the future of agriculture: from sequence to function

WEES lecture by Kevin Foster: The evolution of cooperation and competition in

microbes

WEES lecture by Hanna Kokko: Males exist. Does it matter

WEES lecture by Mart Krupovic: Natural history of viral capsids

WEES seminar by Alexie Papanicolaou: Identification of a new sensory neuron

membrane gene and why phylogenomics is important

2 October, 2017

22 February, 2018

22 January, 2015

19 March, 2015

23 February, 2017

22 June, 2017

WEES seminar by Richard Lenski: Dynamics of Adaptation and Genome Evolution in a Long-Term Experiment

WEES seminar by Frantisek Marec: From sex chromosomes to sex determination in Lepidoptera

Ento-seminar: D.G. Stavenga, Colorful Insect Vision

Ento-seminar: Joop van Loon, Insect Nutrition

Ento-seminar: Eric Schranz, Genetics and Phylogeny

Ento-seminar: Sander Koenraadt, Ecology of vector-borne diseases

Ento-seminar: Chantal Vogels and Helen Esser, Arthropod-borne viruses

Ento-seminar: Katja Poveda, Intra and inter-specific trait variation in arthropods

Seminar by Douglas Mitchell: Genomics-enabled natural products discovery

Seminar by Eric Roalson : Cleomaceae: revising generic concepts and interpreting patterns of adaptation and diversification of $\mathrm{C} 4$ photosynthesis

Seminar by Nicole van Dam: Herbivore-induced plant volatiles accurately reflect evolutionary history, diet breadth, and feeding mode of herbivores

31 August, 2017

25 October, 2017

22 February, 2016

27 June, 2016

29 August, 2016

26 June, 2017

30 October, 2017

15 February, 2018

31 March, 2016

4 May, 2016

17 October, 2016

Seminar by Jennifer Doudna and Edze Westra: Rewriting our genes? CRISPR-CAS systems as tools for genome editing

Keygene seminar: Genotyping in the Seed \& AgroFood Industry: today and in the future

Science Café Wageningen : Agriculture in a changing world

Promega seminar: Optimize and Analyze your RT-qPCR and RNA workflow KeyGene and GENALICE seminar: Generate, Process, and Apply. Solutions for Agrigenomic big data challenges

11th Plant-Insect Interaction Workshop, Leiden

12th Plant-Insect Interaction Workshop, Wageningen

Wageningen PhD symposium: Diversity in Science, Wageningen

EPS Symposium: Goodbye of Ton Bisseling, Wageningen

Mini-Symposium applied Phytopathology: from the lab to the field, Wageningen

Wageningen PhD symposium: Local to Global, Wageningen

Symposium Solanaceae genetic resources in research and breeding, Nijmegen

Symposium on CRISPR-Cas - From Evolution to Revolution, Wageningen

- Seminar plus

- International symposia and congresses

8th European Plant Science Retreat in Barcelona, Spain

16th International Symposium on Insect-Plant relationships in Tours, France

Plant Biotic Stresses \& Resistance Mechanisms in Vienna, Austria

\section{- Presentations}

Green defence Against Pests (GAP) meeting (Oral)

Green defence Against Pests (GAP) meeting (Oral)

Green defence Against Pests (GAP) meeting (Oral)

8th European Plant Science Retreat in Barcelona, Spain (Poster)

Green defence Against Pests (GAP) meeting (Oral)

Green defence Against Pests (GAP) meeting (Oral)

20 - 23 June, 2016

2 - 6 July, 2017

2 - 3 July, 2018

21 April , 2015

25 September, 2015

6 April, 2016

20-23 June, 2016

11 October, 2016

18 May, 2017 


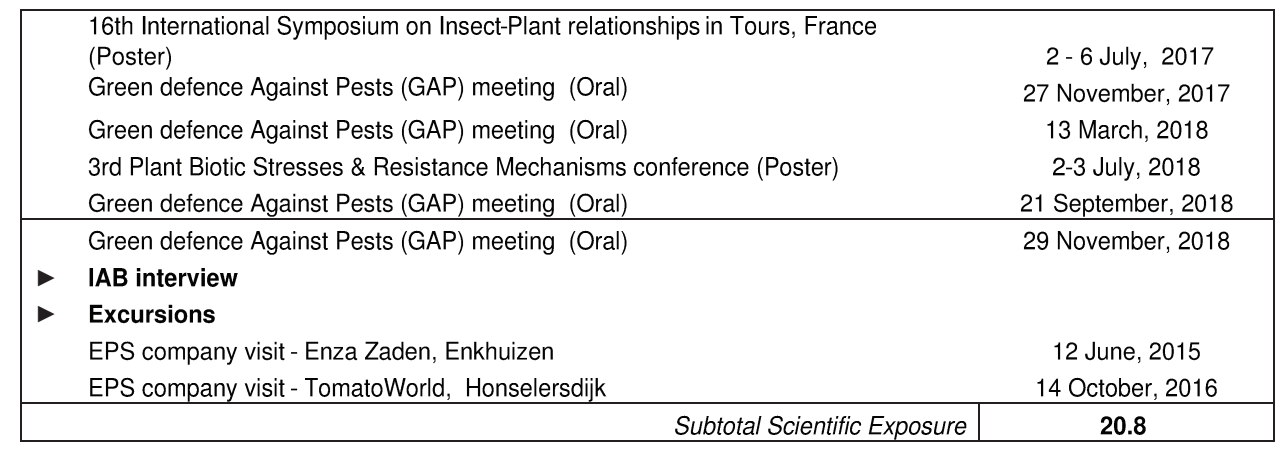

\section{3) In-Depth Studies}

- Advanced scientific courses \& workshops

Genome Assembly, Wageningen Introduction to $\mathrm{R}$ for Statistical Analysis, Wageningen

8th Utrecht International Summer school on Environmental Signaling, Utrecht

The Power of RNA-seq, Wageningen

Basic Statistics, Wageningen

Data Analyses and Visualizations in $\mathrm{R}$

\section{date}

28-29 April, 2015

18 - 19 May, 2015

24-26 August, 2015

10-12 Feb, 2016

14-23 December, 2016

11-12 May, 2017

- Journal club

$\mathrm{PhD}$ journal discussion group, Entomology (Twice a month)

2015-2018

Insect Plant Interactions discussion group, Entomology (Twice a month)

2015-2018

Molecular Ecology \& Evolution discussion group, Entomology (Twice a month)

2017-2018

- Individual research training

Subtotal In-Depth Studies

8.0

\section{4) Personal Development}

- General skill training courses

EPS Introduction Course, Wageningen

Information Literacy PhD including Endnote Introduction, Wageningen

Wageningen Graduate Schools PhD Workshop Carousel, Wageningen

Data Management Planning, Wageningen

Efficient Writing Strategies 2, Wageningen

Last Stretch of the PhD Programme, Wageningen

- Organisation of meetings, $\mathrm{PhD}$ courses or outreach activities

Organised PhD lunch bi-weekly meetings in Entomology, Wageningen

Organised EPS company visit to TomatoWorld

12th Plant-Insect Interaction Workshop, Wageningen

- Membership of EPS PhD Council

EPS PhD council member

\section{date}

22 September, 2015

27-28 October, 2015

8 April, 2016

8 May, 2017

Oct - Dec, 2017

8 December, 2017

$2015-2016$

14 October, 2016

7 November, 2017

2016-2017

${ }^{*}$ A credit represents a normative study load of 28 hours of study. 



\section{Appendix}

The supplementary data of all chapters are available via the link below,

https://www.dropbox.com/sh/ae4su5khxozrgli/AAAj7VAG-Z14j8MfnC0o3SXga?dl=0

The data is organised chapter wise. 
The research described in this thesis was funded by the Netherlands Organisation for Scientific Research through the Perspective programme Green Defences Against Pests (GAP) (NWO, grant no. 13555).

Cover design and Thesis layout: Sandeep J. Sarde

Software back: Tagxedo.com (2019)

Printed by : GVO drukkers \& vormgevers, Ede, The Netherlands 